\title{
TESTING MUK
}

\section{AND ITS PRODUCTS}

FARRINGTON AND WOLL 


$$
\text { 。ํ. }
$$





$$
=
$$









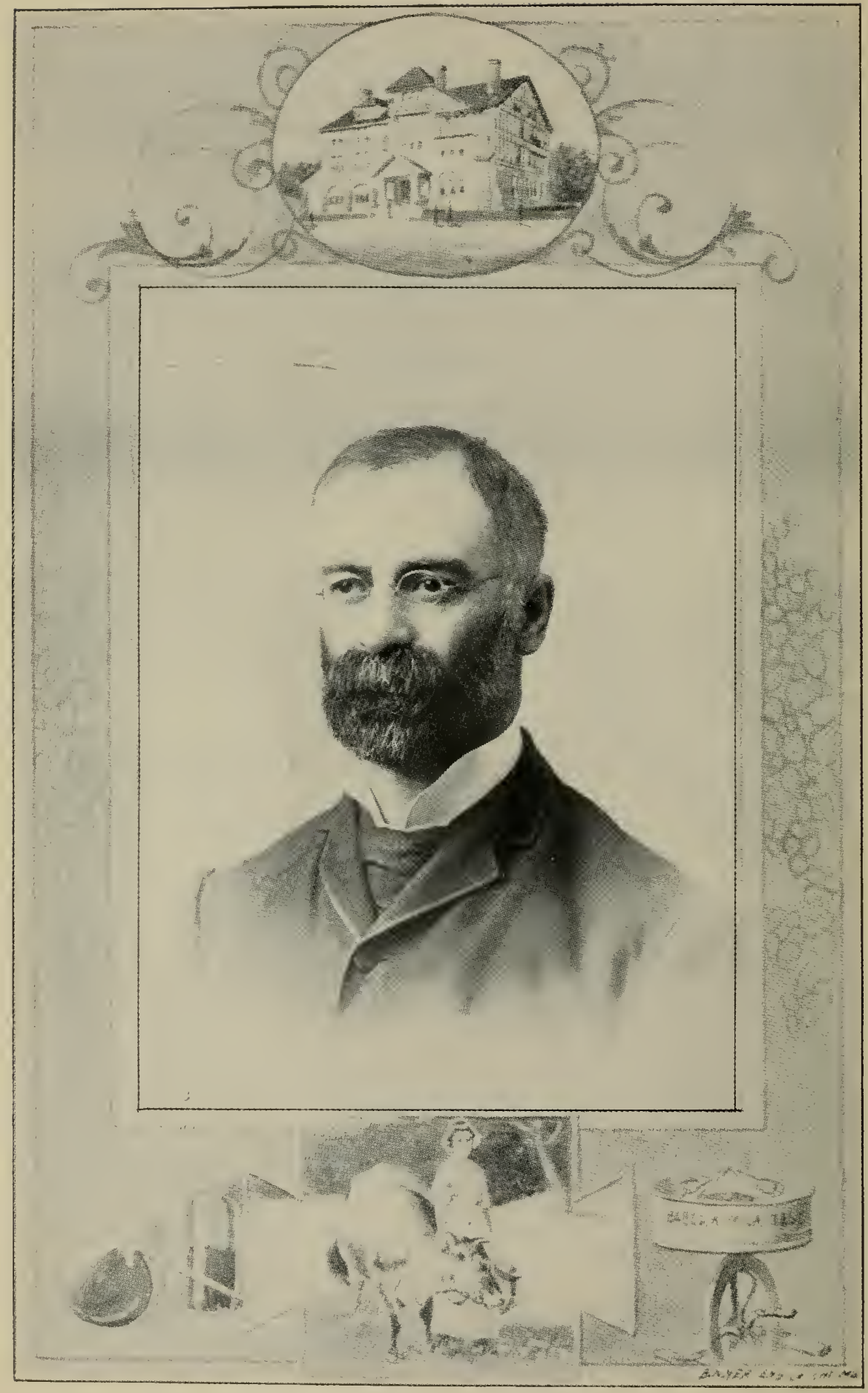

DR. S. M. BABCOCK Inventor of the Babcock Milk Test 


\title{
TESTING MILK \\ AND ITS PRODUCTS
}

\author{
A MANUAL FOR DAIRY STUDENTS, CREAMERY AND \\ CHEESE FACTORY OPERATORS, FOOD CHEMISTS, \\ AND DAIRY FARMERS
}

BY

E. H. FARRINGTON

and

F. W. WOLL

Professor in Charge of Dairy School Professor of Agr'l Chemistry OF THE UNIVERSITY OF WISCONSIN

With Illustrations

TWENTIETH REVISED AND ENLARGED EDITION

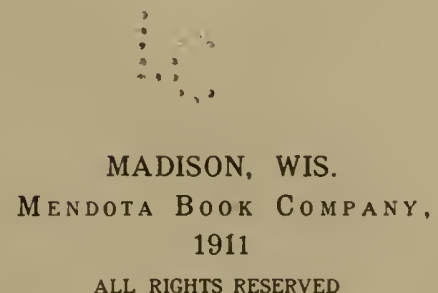

ALL RIGHTS RESERVED 
Copyright, 1897, 1899, 1901, 1904, 1909 and 1911.

B Y E. H. FARRINGTON AND F. W. WOLL
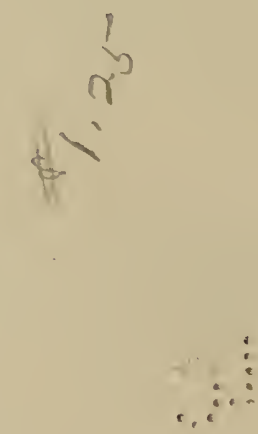

CANTWELL PRINTING COMPANY MADISON, WIS. 


\section{PREFACE TO FIRST EDITION.}

The present volume is intended for the use of dairy students, factory operators, dairymen, food chemists, and others interested in the testing or analysis of milk and its products. The subject has been largely treated in a popular manner; accuracy and clearness of statement, and systematic arrangement of the subject matter have, however, been constantly kept in mind. The aim has been to make the presentation intelligible to students with no further training than a common-school education, but their work will naturally be greatly lightened by the aid of an able teacher.

Complete directions for making tests of milk and other dairy products are given; difficulties which the beginner may meet with are considered in detail, and suggestions offered for avoiding them. It is expected that a factory operator or practical dairyman, by exercising common sense and ordinary care, can obtain sufficient knowledge of the subject through a study of the various chapters of this book to make tests of milk, cream, etc., even if he has had no previous experience in this line.

For the benefit of advanced dairy students who are somewhat familiar with chemistry and chemical operations, Chapter XIV has been added giving detailed instructions for the complete chemical analysis of milk and other dairy products. The detection of preservatives and of artificial butter or filled cheese has also been treated in this connection.

As the subject of milk testing is intimately connected with the payment for the milk delivered at butter-and cheese factories, and with factory dividends, a chapter has been devoted to a discussion of the various systems of factory book-keeping, and tables greatly facilitating the work of the factory secretary or bookkeeper have been prepared and are included in the Appendix.

Madison, Wis., October 1, 1897. 


\section{PREFACE TO TWENTIETH EDITION.}

Each year that passes brings some valuable contributions to our knowledge of the subjects treated in this book and a frequent rerision of it is therefore desirable. The present edition contains descriptions of methods and apparatus that have stood the test of actual use during the past few years; the new information published since the last revision of the book has been carefully sifted, and what was deemed of sufficient importance has been incorporated in such detail as the scope of the book permitted; many changes and additions suggested by the experience of the authors have also been introduced. The book has, in brief, been subjected to a renewed critical examination and careful revision.

The general adoption of the book as a text or reference book in American dairy schools, as well as the favorable reception which it has been accorded by the dairy public in general, will, it is hoped, be further justified by the present revision. Acknowledgment is due to the following parties for loan of electrotypes, viz.: Creamery Plig. Mfg. Co., Chicago, Ill.; Vermont Farm Machine Co., Bellows Falls, Vt.; D. H. Burrell \& Co., Little Falls, N. Y.; Henry Trömner, Philadelphia, Pa.; Torsion Balance Valve Co., New York City; Marschall Dairy Laboratory, Madison, Wis., and International Instrument Co., Canıriage, Mass.

Madison, Wis., Dec. 15, 1910. 


\section{TABLE OF CONTENTS.}

Introduction _......

Chap. I. COMPOSITION OF MILK AND ITS PRODUCTS_._._. 10

Chap. II. SAMPLING MILK _-_._-_._. 23

Chap. III. THE BABCOCK TEST-MILK_............ 2S

A. Directions for mating the test__._._._._._._._. 29

B. Discussion of the details of the test__._._._._._- 37

Chap. IV. THE BABCOCK TEST-CREAM___._._._._. 75

Chap. V. THE BABCOCK TEST-OTHER MILK PRODUC'S __._ 90

Chap. VI. THE LACTOMETER AN DITS APPLICATION_____-_ 102

Chap. VII. TESTING THE ACIDITY OF MILK AND CREAM__._ 119

Chap. VIII. Testing THE PURITY OF IILK_____._. 137

Chap. IX. Testing MILK ON THE FARM _..._........ 142

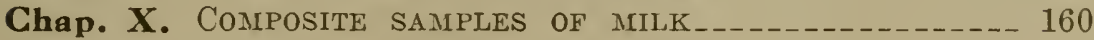

Chap. XI. CREAM TESTING AT CREAMERIES__._._._._. 176

Chap. XII. Calculatioi of ButTer and CHeEse yields _- 187

Chap. XIII. Calculating dividends _............... 203

Chap. XIV. Chemical ANALYSis OF MLK AND ITS PROD-

UCTS___

Appendix _.........

Table I. Composition of milk and its products.

Table II. State and city standards for dairy products.

Table III. Quevenne lactometer degrees corresponding to the scale of the N. Y. Board of Health lactometers.

Table IV. Value of $\frac{100 \mathrm{~S}-100}{\mathrm{~S}}$ for specific gravities from 1.019 to 1.0369 .

Table V. Correction table for specific gravity of milk.

Table VI. Per cent. of solids not fat, corresponding to 0 to 6 per cent. of fat and lactometer readings of 26 to 36 .

Directions for the use of Tables VII, VIII, IX and XI.

Table VII. Iounds of fat in 1 to 1,000 pounds of milk testing 3 to 5.35 per cent.

Table VIII. Pounds of fat in 1 to $1,000 \mathrm{lbs}$. of cream testing 12.0 to 50.0 per cent. fat. 
Table IX. Amount due for butter fat, in dollars and cents, at 12 to $2 \mathrm{~s}$ cents per pound.

Table X. Relative-value tables.

Table XI. Butter chart, showing calculated yield of butter, in pounds, from 1 to 1,000 pounds of milk testing 3.0 to 5.3 per cent. of fat.

Table XII. Overrun table, showing pounds of butter from 100 pounds of milk.

Table XIII. Yield of cheese, corresponding to 2.5 to $6 \mathrm{per}$ cent. of fat, with lactometer readings of 26 to 36 .

Table XIV. Comparisons of Fahreuheit and Centigrade (Celcius) thermometer scales.

Table XV. Comparison of metric and customary weights and measures.

Suggestions regarding the organization of co-operative creameries and cheese factories.

Constitution and by-laws for co-operative factory associations.

Index 


\section{Testing Milk and Its Products.}

\section{INTRODUCTION.}

The need of a rapid, accurate and inexpensive method of determining the amount of butter fat in milk and other dairy products became more and more apparent, in this country and abroa $\bar{d}$, with the progress of the dairy industry, and especially with the growth of the factory system of butter and cheese making during the last few decades. So long as each farmer made his own butter and sold it to private customers or at the village grocery, it was not a matter of much importance to others whether the milk produced by his cows was rich or poor. But as creameries and cheese factories multiplied, and farmers in the dairy sections of our country became to a large extent patrons of one or the other of these, a system of equitable payment for the milk or cream delivered became a vital question.

r. Nearly all the creameries in existence in this country up to about 1890 were conducted on the creamgathering plan: the different patrons creamed their milk by the gravity process, and the cream was hauled to the creamery, usually twice or three times a week, where it was then ripened and churned. The patrons were paid per inch of cream furnished. This quantity was supposed to make a pound of butter, but cream 
from different sources, or even from the same sources at different times, varies greatly in butter-producing capacity, as will be shown under the subject of cream testing $\left(203^{1}\right)$. The system of paying for the number of ereamery inches delivered could not therefore long give satisfaction.

The proposition to take out a small portion, a pint or half a pint, of the cream furnished by each patron, and determine the amount of butter which these samples would make on being churned in so-called test churns, found but a very limited acceptance, on account of the labor involved and the difficulty of producing a first-class article from all the small batches of butter thus obtained.

2. The introduction of the so-called oil test churn in creameries, which followed the creamery-inch system, marked a decided step in advance, and it soon came into general use in gathered-cream factories (202). In this test, glass tubes of about $5 / 8$ inch internal diameter and nine inches long, are filled with cream to a depth of five inches, and the cream is churned; the tubes are then placed in hot water, and the column of melted butter formed at the top is read off by means of a scale showing the number of pounds of butter per creamery inch corresponding to different depths of melted butter. While the oil test is capable of showing the difference between good and poor cream, it is not sufficiently accurate to make' satisfactory distinctions between different grades of good and poor cream. ${ }^{2}$ As a result,

${ }^{1}$ Refers to paragraph numbers.

2 Wis. Expt. Station, bulletin 12 (see also under 203). 
full justice cannot be done to different patrons of creameries where payments for cream delivered are made on the basis of this test.

3. In cheese factories, and since the introduction of the centrifugal cream separator, in separator creameries, the problem of just payment for the milk furnished by different patrons was no less perplexing than in the case of gathered-cream factories. By the pooling system generally adopted, each patron received payment in proportion to the number of pounds of milk delivered, irrespective of its quality. Patrons delivering rich milk naturally will not be satisfied with this system when they find that their milk is richer than that of their neighbors. The temptation to fraudulently increase the amount of milk delivered by watering, or to lower its quality by skimming, will furthermore prove too strong for some patrons; the fact that it was diffcult to prove any fraud committed, from lack of a reliable and practical method of milk analysis, rendered this pooling system still more objectionable.

4. Formerly private dairymen and breeders of dairy cattle who desired to ascertain the butter-producing capacities of the individual cows in their herds were obliged to do this by the cumbersome method of trial churnings : by saving the milk of each cow to be tested, for a day or a week, and churning separately the cream obtained. This requires a large amount of work when a number of cows are to be tested, and can not therefore be done except with cows of great excellence or by farmers having plenty of hired help. Here again the need of a practical milk test was strongly felt. 
5. Introduction of milk tests. The first method which fulfilled all reasonable demands of a practical and reliable milk and cream test was the Babcock test, invented by Dr. S. M. Babcock, of the Wisconsin agricultural experiment station. A description of the test was first published in July, 1890, as bulletin No. 24 of that Station, entitled: $A$ new method for the estimation of fat in milk, especially adapted to creameries and cheese factories. This test, which is now known in all parts of the world where dairying is an important industry, was not, however, the first method proposed for this purpose which could be successfully operated outside of chemical laboratories. It was preceded by a number of different methods, the first one published in this country being Short's method, invented by the late F. G. Short, and described in bulletin No. 16 of Wisconsin experiment station (July 1888).

6. Short's test. In this ingenious method, a certain quantity of milk (20 cc. ${ }^{1}$ ) was boiled with an alkali solution and afterwards with a mixture of sulfuric and acetic acids; a layer of insoluble fatty acids separated on top of the liquid and was brought into the graduated neck of the test bottles by addition of hot water; the reading gave the per cent. of fat in the sample of milk tested.

Short's method did not find very wide application, both because it was rather lengthy and its manipulations somewhat difficult for non-chemists, and because several other methods were published shortly after it had been given to the public.

7. Other milk tests. Of these may be mentioned, besides the Babcock test already spoken of, the Failyer and Willard method, ${ }^{2}$ Parsons' method, ${ }^{3}$ Cochran's test, ${ }^{4}$ the Patrick or Iowa

${ }^{1}$ See 48 , footnote.

${ }^{2}$ Kansas experiment station report, 1888, p. 149.

${ }^{3} \mathrm{~N}$. H. experiment station report, 1888 , p. 69 .

4 Journal of Anal. Chem., III (1889), p. 381. 
station test, ${ }^{1}$ and the Beimling (Leffmann and Beam) test. ${ }^{2}$ Of foreign methods published at about the same time, or previously, the Lactocrite, ${ }^{3}$ Liebermann's method, ${ }^{4}$ the Schmid, ${ }^{5}$ Thörner, ${ }^{8}$ Nahm, ${ }^{7}$ Röse-Gottlieb, ${ }^{8}$ sin-acid method, and the Gerber salmethod ${ }^{10}$ may be noted.

8. All these tests were similar in principle, the solids not fat of the milk being in all cases dissolved by the action of one or more chemicals, and the fat either measured as such in a narrow graduated tube, or brought into solution with ether, gasoline, etc., and a portion thereof weighed on evaporation of the solvent. While this principle is an old one, having been employed in chemical laboratories for generations, its adaptation to practical conditions, and the details as to apparatus and chemicals used were, of course, new and different in each case. The American tests given were adopted to a limited extent within the states in which they originated and even outside of them, as in the case of the Short, Patrick and Beimling methods. The Babcock test, however, soon replaced the different methods mentioned, and during the past twenty years it has now been in almost exclusive use in creameries and cheese factories in this country, where payments are made on the basis of the quality of the milk deliv-

${ }^{1}$ Ia. exp. sta., bull. No. 8, Feb. 1890 ; Iowa Homestead, June 14, 1889.

${ }^{2}$ Vermont exp. sta., bull. No. 21, September, 1890. For description of these and other volumetric methods of milk analysis, see Wiley, Agricultural Analysis, Vol. III, p. 490 et seq; Wing, Milk and its Products, p. 33 et seq., and Snyder, Chemistry of Dairying, pp. 112-113.

${ }^{3}$ Analyst, 1887 , p. 6.

'Fresenius' Zeitschr., 22, 383.

5 Ibid., 27, 464.

- Chem. Centralbl., 1892, 429.

7 Milch-Zeitung, 1894, No. 35 ; 1897, No. 50.

${ }^{8}$ Landw. Vers. Stat., 40, 1.

Milch-Zeitung, 1904, No. 27 .

10 Milch-Zeitung, 1906, No. 8. 
ered, as well as in the routine work in experiment station laboratories, and among milk inspectors and private dairymen.

9. The Babcock test. The main cause why the Babcock test has replaced all competitors is doubtless to be sought in its simplicity and its cheapness. It has but few manipulations, is easily learned, and is cheap, both in first cost and as regards running expenses.

The test is furthermore speedy, accurate ${ }^{1}$ and easily applied under practical conditions, and may therefore safely be considered the best milk test available at the present time.

The method is applicable not only to whole milk, but to cream, skim milk, butter milk, whey, condensed milk, and (if a small scale for weighing out the sample is available) to cheese and butter. ${ }^{2}$

With all its advantages, the Babcock milk test is not in every respect an ideal test. The handling of the very corrosive sulfuric acid requires constant care and attention; the speed of the tester, the strength of the acid, the temperature of the milk to be tested, and other points, require constant watching, lest the results obtained be too low or otherwise unsatisfactory. In the hands of careful operators the test can, however, always be relied upon to give most satisfactory results.

Io. Foreign methods. In European countries five practical milk and cream tests, besides the Babcock test,

${ }^{1}$ For a summary of comparative analyses made by the Babcock test and gravimetric analysis up to 1892, see Hoard's Dairyman, Oct. 7, 1892, p. 2560 ; also Schrott-Fiechtl, Milchzeitung, 1896, p. 183 et seq.

2 The Babcock test, like the ether-extraction method gives, however, somewhat too low results in the case of skim milk (97). 
are in use at the present time, viz.: Gerber's acidbutyrometer, the sin-acid (or no-acid) test, the lactocrite. De Laval's butyrometer, and Fjord's centrifugal cream test. ${ }^{1}$

Of these tests the last one has never, to ourknowledge, been introduced into this country, and the first four only to a limited extent.

\section{The Gerber method} (fig. 1) is essentially the old Beimling method (7), worked out independently by the Swiss chemist, Dr. N. Gerber. In this test sulfuric acid of the same strength as in the Babcock test is used, and a small quantity of amyl alco-
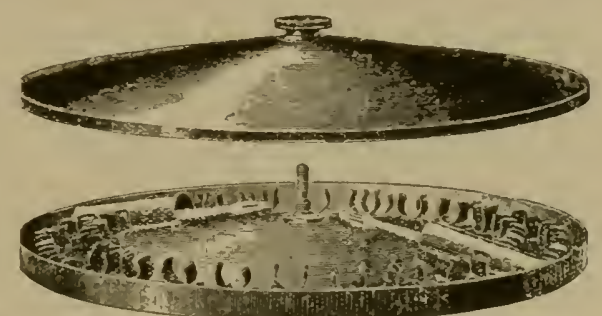

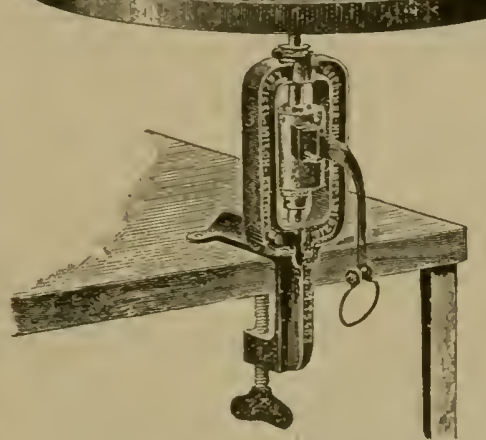

FIG. 1. 'The Gerber' acidbuty rometer. hol is added. The amyl alcohol facilitates the separation of the fat, but may introduce a source of error on account of impurities contained therein, when the results obtained with a new lot of alcohol can not be checked against gravimetric analysis or against tests made with amyl alcohol known to give correct results. This method is, however, extensively used in European countries, having there practically replaced the Babcock test or been adopted in preference to it.

11a. The sin-acid test was inrented by the German chemist A. Sichler and published in $1904 .^{3}$ In place of the sulfuric acid used in the Babcock and Gerber tests, Sichler employs a solution of

1 The Listcr-I3abcock milk test advertised in English papers and known as such in England, is the regular Babcock test, to which the English manufacturers bave affixed their name; the same applies to the Ahlborn-Babcock and the Krugmann-Babcock methods.

${ }^{2}$ Gerber, Die praktische Milchprüfung, 7 th edition, 1900.

${ }^{3}$ Milchztg., 1904, p. 417. The rord $\sin$ (sine) is Latin and means without; hence, when introduced into this country in 1909 the method was called the no-acid test. 
Rochelle salts, sodium sulfate and sodium hydroxid. ${ }^{1} 150$ ec. of this mixture of salts are dissolved in 1 liter of water. In testing milk, 11 cc. of this solution and $0.6 \mathrm{cc}$. of "sinol" (isobutylalcohol) are added to 10 cc. of milk. After thorough mixing of the milk and solution the test bottles are placed in water of $113^{\circ} \mathrm{F}$. for $3-5$ minutes, when they are shaken till all the curd dissolves. They are then revolved in a centrifuge for 3 minutes and the results read off. By heating the bottles for 1 hour in boiling hot water correct results may be obtained without the use of a centrifuge. The main advantages of the method appear to lie in this fact and in that the use of a corrosive acid is avoided.

12. The Lactocrite was one of the earliest practical milk tests introduced. It was invented by De Laval in 1886. The acids used in this test are lactic acid (originally, acetic acid) with a mixture of hydrochloric and sulfuric acids. This test is now but rarely met with.

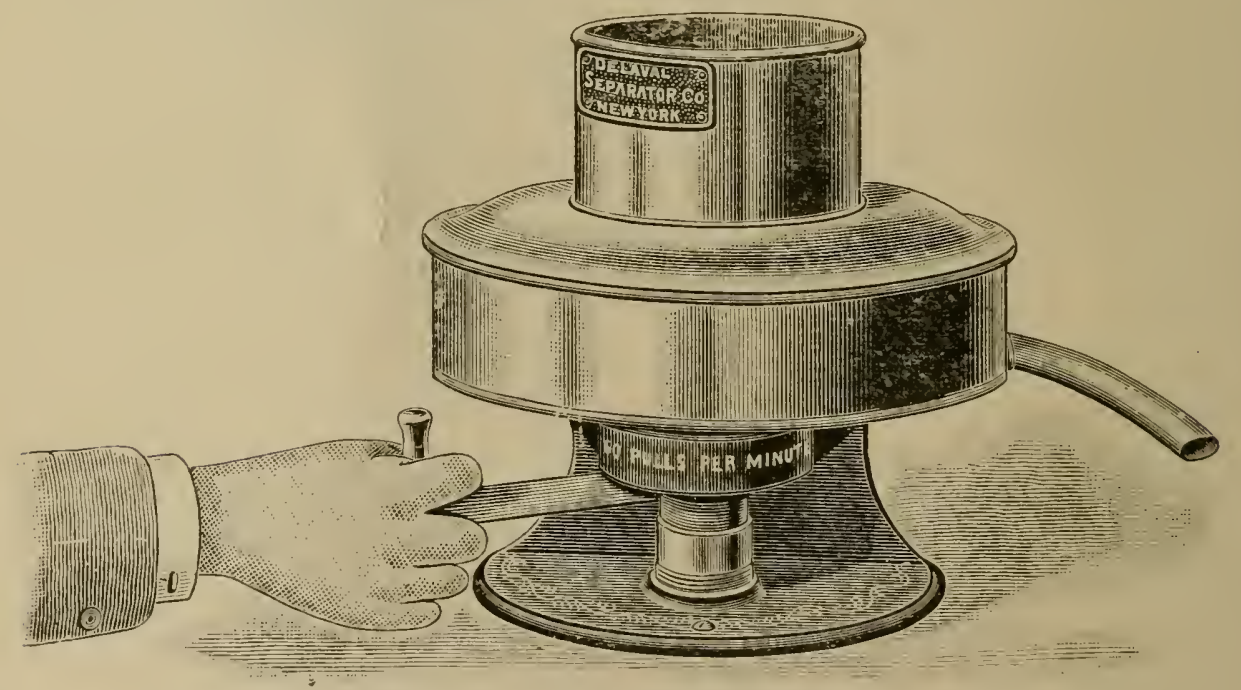

Fig. 2. De Laval's butyrometer.

13. In the De Laval butyrometer (fig. 2) the same acid is used as in the Babcock test, but the tubes employed and the manipulations of the method differ materially from this test; a smaller sample of milk is taken (only 2 cc.) and a correspondingly small quantity of acid used. Where a large number of

${ }^{1}$ Barthel-Goodwin, Methods used in Examination of Milk and Dairy Products, p. 77. 
milk samples are tested every day, as, for instance, in milk control stations, the butyrometer may be preferable to the Babcock test; but it requires more skill of the operator and does not work satisfactorily in case of sour, loppered, or partially churned milk.

14. Fjord's centrifugal cream tester ${ }^{1}$ (fig. 3 ) is extensively used in Denmark and is mentioned in this conncetion as it furnishes, as a rule, a reliable method for comparing the quality of different lots of milk. The method was published in 1878, by the late N. J. Fjord, director of the state experiment station in Copenhagen, through whose exertions and on whose authority it was introduced into Danish creameries in the middle of the eighties. No chemicals are added in this test, the milk being simply placed in glass tubes, seven inches long and about twothirds of an inch in diameter, and whirled for twenty minutes at a rate of 2000 revolutions per minute at $55^{\circ} \mathrm{C}\left(131^{\circ} \mathrm{F}\right.$.). The reading of the cream layer thus obtained gives the per cent. of cream, and not of butter fat, in the sample tested. One hundred and ninety-two samples of milk can be tested simultaneously. Within the limits of normal Danish herd milk, the results obtained correspond to the per cents of fat present in the samples, one per cent. of cream being equal to about 0.7 per cent. of fat; outside of these limits the test

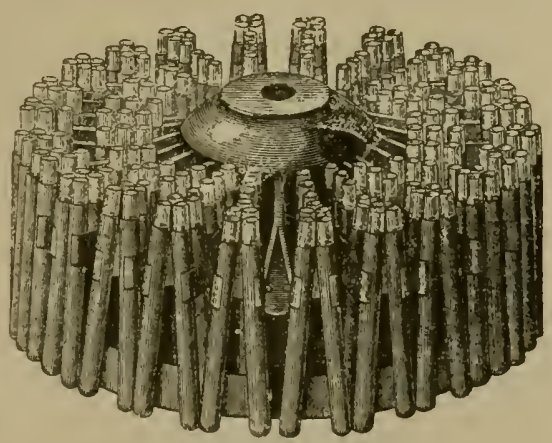

FIG. 3. Fjord's centrifugal cream tester.

is, however, unreliable, especially in case of very rich milk and strippers' milk. Only sweet milk can be tested by this method. Milk tests proper, like the Gerber, Babcock and De Laval tests, have during recent years been introduced into Denmark and are used in some creameries. ${ }^{2}$

\footnotetext{
${ }^{1}$ State Danish experiment station, Copenhagen, sixth and ninth reports, $1885-7$.

${ }^{2}$ Among foreign milk tests in use abroad should also be mentioned the Lindstrom butyrometer and the Wollny refractometer, both of which, in the hands of trained chemists, may prove better adapted for use where a very large number of samples are to be tested at a time. than any other available milk test.
} 


\section{CHAP'TER I.}

\section{COMPOSITION OF MILK AND ITS PRODUCTS.}

Before taking up the discussion of the Babcock milk test, a brief description of the chemistry of milk and its products is given, so that the student may understand what are the components of dairy products, and the relation of these to each other. Only such points as have a direct bearing upon the subject of milk testing and the use of milk tests in butter and cheese factories or private dairies will be treated in this chapter, and the reader is referred to standard works on dairying for more detailed information in regard to the composition of dairy products.

15. Composition of milk. Milk is composed of the following substances: water, fat, casein, albumen, milk sugar, and ash. A few other substances are present in small quantities, but they are hardly of any practical importance and will not be considered here. The components of the milk less the water are known collectively as milk solids or total solids, and the total solids less the fat, i. e., casein, albumen, milk sugar, and ash, are often spoken of as solids not fat or the non-fatty milk solids. The milk serum includes all components of the milk less the fat; the serum solids are therefore another name for the solids not fat; when given, they are, however, generally calculated to per cent. of milk serum, not of milk. If, e. g., a sample of milk contains 
nine per cent. of solids not fat, and three per cent. of fat, the milk serum will make up 97 per cent. of the milk, and the serum solids, $\frac{9 \times 100}{97}=9.28$ per cent. of the milk serum.

16. Water. The amount of water contained in cow's milk ranges from 82 to 90 per cent. Normal cow's milk will not, as a rule, contain more than 88 per cent. of water, nor less than 84 per cent. In states where there are laws regulating the sale of milk, as is the case in eighteen states of the Union (see Appendix, Table II), the maximum limit for water in milk in all instances but one (South Carolina) is 88 per cent.; the state mentioned allows 88.5 per cent. of water in milk offered for sale within her borders. 'The effect of fraudulently increasing the water content of millk by watering is considered under Adulteration of Milk (121).

17. Fat. The fat in milk is not in solution, but suspended as very minute globules, which form an emulsion with the milk serum; the globules are present in immense numbers, viz., on the average about one hundred millions in a single drop of milk; a quart of milk will contain about two thousand billions of fat globules, a number written with thirteen figures. The sizes of the globules in the milk from the same cows vary according to the stage of the period of lactation, the globules being largest at the beginning of the lactation period, and gradually decreasing in size with its progress. Different breeds of cows have fat globules of different average sizes; the Channel Island cows are thus noted for the relatively large fat globules of their 
milk, while the lowland breeds, the Ayrshire, and other breeds have uniformly smaller globules. The diameter of average sized fat globules in fresh milkers is about .004 millimeter, or one six-thousandth of an inch; that is, it takes about six thousand such globules placed side by side to cover one inch in length. The globules of any sample of milk vary greatly in size; the largest globules are recovered in the cream when the milk is set or run through a cream separator, and the smallest ones remain in the skim milk; thoroughly skimmed separator skim milk contains only a small number of very minute fat globules.

Milk fat is composed of so-called glycerides of the fatty acids, i. e., compounds of the latter with glycerin; some of the fatty acids are insoluble in water, viz., palmitic, stearic, and oleic acids, while others are soluble and volatile, the chief ones among the latter being butyric, caprylic, and capronic acids. The glycerides of the insoluble fatty acids make up about 92 per cent. of the pure milk fat; about 8 per cent. of the glycerides of volatile fatty acids are therefore found in natural milk- (and butter-) fat. The distinction between natural and artificial butter lies mainly in this point, since artificial butter (butterine, oleomargarine) as well as other solid animal fats contain only a very small quantity of volatile fatty acids. The glycerides of the volatile fatty acids are unstable compounds, and are easily decomposed through the action of bacteria or light; the volatile fatty acids thus set free, principally butyric acid, are the cause of the unpleasant odor met with in rancid butter. 
Cow's milk generally contains between three and six per cent. of fat; in American milk we find, on the average, toward four per cent. of fat. The milk from single cows in perfect health will occasionally go below or above the limits given, but mixed herd milk rarely falls outside of these limits. The standard adopted by the U. S. government for fat in milk is 3.25 per ct. The legal standard for fat in milk in most states of the Union is 3 per cent.; Rhode Island allows milk containing 2.5 per cent. of fat to be sold as pure, while Georgia and Minnesota require it to contain 3.5 per cent., and Massachusetts 3.7 per cent. (in the months of May and June; see Appendix, Table II).

18. Casein and albumen. These belong to the socalled nitrogenous substances, distinguished from the other components of the milk by the fact that they contain the element nitrogen. Another name is albuminoids or protein compounds. Casein is precipitated by rennet in the presence of soluble calcium salts, and by dilute acids and certain chemicals; albumen is not acted upon by these agents, but is coagulated by heat, a temperature of $170^{\circ} \mathrm{F}$. being sufficient to effect a perfect coagulation. The casein, fat, and water, are the main components of nearly all kinds of cheese. In the manufacture of cheddar and most other solid cheeses, the casein is coagulated by rennet, and the curd thus formed holds fat and whey mechanically, the latter containing in solution small quantities of non-fatty milk solids. The albumen goes into the whey and is lost for cheese making; in some countries it is also made into cheese by evaporating the whey under constant 
stirring; whole milk of cows or goats is often added and incorporated into such cheese (primost, gjetost).

Casein is present in milk partly in solution, in the same way as milk sugar, soluble ash-materials and albumen, and partly in suspension, in an extremely fine colloidal condition, mixed or combined with insoluble calcium phosphates. The casein and calcium phosphates in suspension in milk may be retained on a filter made of porous clay (so-called Chamberland filters).

About 80 per cent. of the nitrogenous compounds of normal cow's milk are made up of casein; the rest is largely albumen. If the amount of casein in milk be determined by precipitation with rennet or dilute acids, and the albumen by boiling the filtrate from the casein precipitate, it will be found that the sum of these two compounds do not make up the total quantity of nitrogenous constituents in the milk. The small remaining portion (about five per cent. of the total nitrogenous constituents) has been called by various authors, globulin, albumose, hemi-albumose, nuclein, nucleon, proteose, etc. The nitrogenous constituents of milk are very unstable compounds, and their study presents many and great difficulties; as a result we find that no two scientists who have made a special study of these compounds agree as to their properties, aside from those of casein and albumen, or their relation to the nitrogenous substances found elsewhere in the animal body. For our purpose we may, however, consider the nitrogen compounds of milk as made up of casein and albumen, and the term casein and albumen, as used in this book, is meant to include the total nitrogenous constituents of 
milk, obtained by multiplying the total nitrogen content of the milk by $6.25 .^{1}$

The quantity of casein in normal cow's milk will vary from 2 to 4 per cent., and of albumen, from .5 to .8 per cent. The total content of casein and albumen ranges between 2.5 and 4.6 per cent, the average being about 3.2 per cent. Milk with a low fat content will contain more casein and albumen than fat, while the reverse is generally true in case of milk containing more than 3.5 per cent. of fat.

19. Milk sugar or lâctose belongs to the group of organic compounds known as carbohydrates. It is a commercial product manufactured from whey and is obtained in this process as pale white crystals, of less sweet taste and less soluble in water than ordinary sugar (cane sugar, sucrose). About 70 per cent. of the solids in the whey, and 33 per cent. of the milk solids, are composed of milk sugar.

When milk is left standing for some time, viz., from one to several days, according to the temperature of the surrounding medium it will, as a rule, turn sour and soon becomes thick and loppered. This change in the composition.and appearance of the milk is brought about through the action of acid-forming bacteria on the milk sugar. These are present in ordinary milk in immense numbers, and under favorable conditions of temperature multiply rapidly, feeding on the milk sugar

${ }^{1}$ The factor 6.25 is generally used for obtaining the casein and albumen from the total nitrogen in the milk, on the theory that protein compounds contain $16 \% \mathrm{~N}$.; the factor 6.37 would, however, be more correct, since casein and albumen, according to our best authorities, contain on the average 15.7 per cent of nitrogen $\left(\frac{1}{1} \frac{0}{5} .7=6.37\right)$ 
as they grow, and decomposing it into lactic acid. When this change alone occurs, there is not necessarily a loss in the nutritive value of the milk, since milk sugar breaks up directly into lactic acid. This is shown by the following chemical formula:

\section{$\mathrm{C}_{12} \mathrm{H}_{22} \mathrm{O}_{11} \mathrm{H}_{2} \mathrm{O}$ (lactose) $=4 \mathrm{C}_{3} \mathrm{H}_{6} \mathrm{O}_{3}$ (lactic acid). ${ }^{1}$}

Ordinarily the souring of milk is, however, more complicated, and other organic bodies, like butyric acid, alcohol, etc., and gases like carbonic acid are formed, resulting in a loss in the feeding value of the milk. While sour milk may therefore contain a somewhat smaller proportion of food elements than sweet milk, it will generally produce better results when fed to farm animals, especially pigs, than is obtained in feeding similar milk in a sweet condition. The cause of this may lie in the stimulating effect of the lactic acid of sour milk on the appetites of the animals, or in its aiding digestion by increasing the acidity of the stomach juices.

That the souring of milk is due to the activities of bacteria present therein is shown clearly by the fact that sterile milk, i. e., milk in which all germ life has been killed, will remain sweet for any length of time when kept free from infection.

The amount of milk sugar found in normal cow's milk varies from 3.5 to 6 per cent., the average content being about 5 per cent.; in sour milk this content is decreased to toward 4 per cent.

${ }^{1}$ One molecule of milk sugar is composed of 12 atoms of carbon (C), 22 atoms of hydrogen $(H), 11$ atoms of oxygen $(\mathrm{O})$, and one molecule of water $\left(\mathrm{H}_{0} \mathrm{O}\right)$. In the same way, the lactic acid molecule consists of 3 atoms of carbon, 6 atoms of hydrogen, and 3 atoms of oxygen. 
20. Ash. The ash or mineral substances of milk are largely composed of chlorids and phosphates of sodium, potassium, magnesium and calcium; iron oxid and sulfuric and other acids are also present in small quantities among the normal mineral milk components. The amounts of the different bases and acids found in milk ash have been determined by a number of chemists; the average figures obtained are given in the following table, calculated per 100 parts of milk (containing .75 per cent. of ash) and per 100 parts of milk ash.

\section{Mineral Components of Milk.}

In per cent of mill. In per cent of ash.

Potassium oxid $\left(\mathrm{K}_{2} \mathrm{O}\right)$-...-.-. .19 per ct.

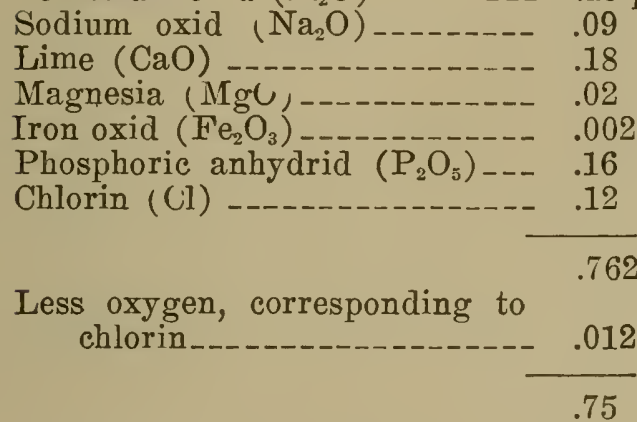

25.64 per ct.

12.45

24.58

3.09

.34

21.24

16.34

103.68 per ct.

3.68

100.00

The combinations in which the preceding bases and acids are contained in the milk are not known with certainty. According to Söldner, 36 to 56 per cent. of the phosphoric acid found in milk, and from 53 to 72 per cent. of the lime, are present in suspension in the milk as di- and tri-calcium phosphates, and may be filtered out by means of Chamberland filters (18), or by long continued centrifuging $\left(\mathrm{Babcock}^{1}\right)$. The rest of the ash constituents are dissolved in the milk serum.

1 Wisconsin experiment station report 12, p. 93 . 
The ash content of normal cow's milk varies but little, as the rule only between .6 and .8 per cent, with an average of .7 per cent. Milk with a high fat content generally contains about .8 per cent. of ash; strippers' milk always has a high ash content, at times even exceeding one per cent. Ordinarily, the mineral constituents are least liable to variations of any of the components of the milk.

2I. Other components. Besides the milk constituents enumerated and described in the preceding pages, normal milk contains a number of substances which are present in but small quantities and have only scientific interest, such as the milk gases (carbonic acid, oxygen, nitrogen), citric acid, lecithin, cholesterin, urea, hypoxanthin, lactochrome, etc.

22. Average composition. The average percentage composition of cow's milk will be seen from Table I in the Appendix. The following statement shows the limits within which the components of normal American cow's milk are likely to come:

\begin{tabular}{|c|c|c|c|}
\hline & Minimum. & Maximum. & Averagc. \\
\hline $\begin{array}{l}\text { Water } \\
\text { Fat }\end{array}$ & $\begin{array}{l}52.0 \text { per ct. } \\
2.3\end{array}$ & $\begin{array}{l}90.0 \text { per ct. } \\
7.8\end{array}$ & $\begin{array}{l}\text { s1.4 per cl. } \\
3.7\end{array}$ \\
\hline Casein and albumen & 2.5 & 4.6 & 3.2 \\
\hline Milk sugar _._._. & 3.5 & 6.0 & 5.0 \\
\hline Ash & .6 & .9 & .7 \\
\hline
\end{tabular}

23. Colostrum milk. The liquid secreted directly after parturition is known as colostrum milk or biestings. It is a thick, yellowish, viscous liquid; its high content of albumen and ash is characteristic, and also its low content of milk sugar. Owing to the large quantity of albumen which colostrum contains, it will coagu- 
late on being heated toward the boiling point. In the course of four or five days the secretion of the udder gradually changes from colostrum to normal milk; the milk is considered fit for direct consumption or for the manufacture of cheese and butter, when it does not coagulate on boiling and is of normal appearance as regards color, taste, and other properties. For composition of colostrum milk, see Appendix, Table I.

24. Composition of milk products. In addition to its use for direct consumption, milk is the raw-material from which crcam, butter, cheese, and condensed milk are obtained.

When milk is left standing for some time or subjected to centrifugal force, it will separate into two distinct parts, cream and skim milk. The proportion of each part which is obtained, and their chemical composition, will depend on the method by which the separation is effected; in the so-called gravity process where the cream is separated on standing-either in shallow pans in the air, or in deep cans, submerged in cold water-a less complete separation is reached, since the skim milk obtained is richer in fat than when the separation takes place through the action of centrifugal force.

In modern creameries the milk is now al ways skimmed by means of cream separators. Separator cream will contain from 15 to 50 per cent. of fat, according to the adjustment of the separator and of the milk supply; ordinarily it contains about 25 per cent. of fat. Cream of average quality, in addition to the fat content given, consists of about 66 per cent. of water, 3.8 per cent. 
casein and albumen, 4.3 per cent. milk sugar, and .5 per cent. ash.

The skim milk is made up of the milk serum (15) and a small amount of fat, viz., toward .4 per cent. when obtained by the gravity process, and less than .2 per cent. in the case of separator skim milk. Milk set in shallow pans in the air, or in deep cans in water above $60^{\circ} \mathrm{F}$., will give skim milk containing one-half to over one per cent. of fat. Skim milk is used as a food for young farm animals or as human food, and in this country only in exceptional cases, for the manufacture of cheese.

25. Cream is used for the manufacture of butter or for direct consumption. In the former case a certain amount of acidity is generally allowed to develop therein previous to the churning process. This secures a more complete churning and produces peculiar flavors in the butter, without which it would seem insipid to most people. Nearly all butter made in this country is salted before being placed on the market. Salt is a preservative and for a limited length of time prevents butter from spoiling. Unsalted butter made from sweet cream is a common food article in Southern and Middle Europe, but only an insignificant amount is manufactured and consumed in America; salted butter made in Europe also contains considerably less salt than American butter (see Appendix, Table I). Butter contains all the fat of the cream except a small portion which goes into the butter milk, and a small unavoidable mechanical loss incident to the handling of the products. Butter should contain at least 80 per 
cent. of fat and ordinarily contains about 83 per cent.; besides this amount of fat, butter is generally composed of about 13 per cent water, 1 per cent curd and lactic acid, and 3 per cent salt.

Butter milk has a composition similar to skim milk, but varies much more than this product, according to the acidity, temperature, and thickness of the cream, and other churning factors. It contains about 9 per cent. of solids, viz., milk sugar (and lactic acid) 4 per cent., casein and albumen 4 per cent., fat . 3 per cent., and ash .7 per cent.

26. The quantities of butter and by-products obtained in the manufacture of butter are as follows: $1000 \mathrm{lbs}$. of milk of average quality will give about $850 \mathrm{lbs}$. of skim milk and $145 \mathrm{lbs}$. of cream (separator slime and mechanical loss, 5 lbs.); this amount of cream will make about $42 \mathrm{lbs}$. of butter and $100 \mathrm{lbs}$. of butter milk (mechanical loss, 3 lbs.).

27. In the manufacture of American cheddar cheese, whole milk is heated to about $86^{\circ} \mathrm{F}$., and a small amount of rennet extract is added, which coagulates the casein; the albumen of the milk is not precipitated by rennet and remains in solution (18). "Green" cheese, as taken from the press, is made up, roughly speaking, of 37 per cent. of water, 34 per cent. of fat, 24 per cent. of albuminoids (nearly all casein), and about 5 per cent. of milk sugar, lactic acid, and ash (largely salt). In the curing of cheese there is some loss by drying, but the main changes occur in the breaking up of the firm curd into soluble and digestible nitrogenous compounds, peptones, amids, etc. 
Whey is the by-product obtained in the manufacture of cheese. It consists of water and less than 7 per cent. of solids; of the latter about 5 per cent. is milk sugar, .8 per cent. albumen, .6 per cent. ash, and .3 per cent. fat. Whey is generally used for feeding farm animals; it is the raw-material from which milk sugar and whey cheese are made.

28. Condensed milk is manufactured from whole milk or from partially skimmed milk. In many brands a large quantity of sugar ( 25 per cent. or more) is added to the condensed milk in the process of manufacture so as to secure perfect keeping quality in the product. Brands to which no sugar has been added are also on the market, and in case of such brands the relation between the various solid constituents of the condensed milk will be essentially the same as that between the constituents of milk solids. Condensed milk should contain at least $8 \%$ fat, and must be free from preservatives and other foreign substances (except sugar).

Tables are given in the Appendix showing the average composition of the various milk products.

\section{Questions.}

1. What is the average composition of cow's milk; state briefly the properties of the various constituents.

2. What is meant by total solids; solids not fat; milk serum; serum solids?

3. What is colostrum milk Give its average composition, and in what particulars it mainly differs from normal milk.

4. Give the average composition of cream, skim milk, buttermilk, whey, butter and cheddar cheese.

5. Explain the distribution of the components of milk in (a) butter-making, (b) cheese-making. 


\section{CHAP'TER II.}

\section{SAMPLING MILK.}

29. The butter fat in milk is not in solution, like sugar dissolved in water, but the minute fat globules or drops, in which form it occurs, are held in suspension in the milk serum (17). Being lighter than the serum, the fat globules have a tendency to rise to the surface of the milk. If, therefore, a sample of milk is left standing for even a short time, the upper layer will contain more fat than the lower portion. This fact should always be borne in mind when milk is sampled. The rapidity with which fat rises in milk can be easily demonstrated by allowing a quantity of sweet milk to stand in a cylinder or a milk can for a few minutes, and testing separately the top, middle and bottom layer of this milk.

The amount of mixing necessary to evenly distribute the constituents of milk throughout its mass may be ascertained by adding a few drops of cheese color to a quart of milk. The yellow streaks through the milk will be noticed until it has been poured several times from one vessel to another, when the milk will have a uniform pale yellow color. Stirring with a stick or a dipper will not produce an even mixture so quickly or so completely as pouring the milk a few times from one vessel to another. In sampling milk for testing it should always be mixed just before the milk is measured into the bottle; if several tests are made of a sample, the milk should be mixed before each sampling. 
30. Partially churned milk. A second difficulty sometimes met with in sampling whole milk arises from the fact that a part of the butter fat may be separated in the form of small butter granules, by too zealous mixing or by reckless shaking in preparing the sample for testing: This will happen most readily in case of milk from fresh cows or milk containing exceptionally large fat globules. When some of the butter granules are thus churned out, they quickly rise to the surface of the milk after pouring and cannot again be incorporated in the milk by simple mixing; it is, therefore impossible to obtain a fair sample of such milk for testing without taking special precautions which will be explained in the following. The granules of butter may be so small as to pass into the pipette with the milk and the quantity measured thus contain a fair proportion of them, but they will be found sticking to the inside of the pipette when this is emptied, and thus fail to be carried into the test bottle with the milk.

A similar partial churning of the milk will sometimes take place in the transportation cans. When such milk is received at the factory, the butter granules are caught by the strainer cloth through which the milk is poured, and are thus lost both to the factory and to the farmer. This separated fat cannot be added to the cream or to the granular butter, without running the risk of making mottled butter, and it will not enter into the sample of milk taken for testing purposes.

When milk samples are sent by mail or express in small bottles, or carried to the place of testing, they often arrive with lumps of butter floating in the milk or sticking to the glass. This churning of the milk can 
be easily prevented by completely filling the bottle or the can. If there is no space left for the milk in which to splash around, the fat will not be churned out in transit.

31. Approximately accurate results may generally be obtained with a partially churned sample of milk, if a teaspoonful of ether be added to it. After adding the ether, cork the bottle and shake it until the lumps of butter are dissolved. This ether solution of the butter will mix with the milk and from the mixture a fairly satisfactory sample may generally be taken. The dilution of milk by the ether introduces an error in the testing, and only the smallest quantity of ether necessary to dissolve the lumps of butter should be used. If desired, a definite quantity of ether, say five per cent. of the volume of the sample of milk to be tested, may be added; in such cases the result of the test must be increased by the per cent. of ether added.

EXAMPLE.-To a 4-oz. sample (120 cc.) of partially churned milk, 5 per cent, or 6 cc., of common ether are added; the mixture gave an average test of 4.2 per cent. The test must be increased by $\frac{5}{100} \times 4.2=.21$ per cent., and the original milk therefore contained $4.2+.21=4.41$ per cent. of fat.

Milk containing ether must be mixed cautiously with acid in making a test, so as to aroid a loss of the contents of the bottle by the sudden boiling of the ether due to the heat evolved in mixing the milk and the acid.

Instead of adding ether to partially churned samples, the milk may be heated to about $110^{\circ} \mathrm{F}$. for a few minutes, so as to melt the butter granules; the sample is now shaken vigorously until a uniform mixture of milk and melted butter is obtained, and a pipetteful is then quickly drawn from the sample. 
32. Sampling sour milk. When milk becomes sour, the casein is coagulated and the mechanical condition of the milk thereby changed so as to render difficult a correct sampling. The butter fat is not, however, changed in the process of souring; this has been shown by one of us, among others, in a series of tests which were measured from one sample of sweet milk into six test bottles. A test of the milk in one of these test bottles was made every month for six months, and approximately the same amount of fat was obtained in the tests throughout the series, as was found originally in the milk when tested in a sweet condition. ${ }^{1}$ If the milk is in condition to be sampled, its souring does not therefore interfere with its being tested by the Babcock test or with the accuracy of the results obtained.

In order to facilitate the sampling of sour or loppered milk, some chemical may be added which will redissolve the coagulated casein and produce a uniform mixture that can be readily measured with a pipette. Any alkali (powdered potash or soda, or liquid ammonia) will produce this effect. Only a very small quantity of powdered alkali is necessary for this purpose. The complete action of the alkali on sour milk requires a little time, and the operator should not try to hasten the solution by adding too much alkali. An excess of alkali will often cause such a violent action of the sulfuric acid on the milk to which the acid is added (on account of the heat generated or the presence of car-

${ }^{1}$ See Hoard's Dairyman, April 8, 1892. The same holds true for cream, as shown by Winton (U. S. Dept. Agr., Div. of Chemistry, bull. 43 , p. 112). As to length of time Babcock tests will keep, see Vt. exp. sta., bull. 106. 
bonates in the alkali) that the mixture will be thrown out of the neck of the test bottle when this is shaken in mixing the milk and the acid (37). When powdered alkali is added to the milk, it should be allowed to stand for a while, with frequent shaking, until the curd is all dissolved and an even translucent liquid is obtained. Such milk may become dark-colored by the action of the alkali, but this color does not interfere with the accuracy of the test.

Instead of powdered soda or potash, these substances dissolved in water (soda or potash lye), or strong ammonia, may be used for the purpose of dissolving the coagulated casein in sour milk. In this case, a definite proportion of alkali solution must be taken, however, 5 per cent. of the volume of milk being usually sufficient, and the results obtained are increased accordingly.

33. Sampling frozen milk. When milk freezes, it separates into two distinct portions: Milk crystals, largely made up of water, with a small admixture of fat and other solids, and a liquid portion, containing nearly all the solids of the milk. In sampling frozen milk it is therefore essential that the liquid and the frozen part be warmed and thoroughly mixed by pouring gently back and forth from one vessel into another; the sample is then taken and the test proceeded with in the ordinary manner (36).

\section{Questions.}

1. What precautions must be taken in sampling milk Give reasons.

2. How can a fair sample be taken of (a) partially churned milk, (b) sour milk, (c) frozen milk

3. If 15 cc. of ammonia are added to 500 cc. of sour milk, and a test of 3.45 obtained, what is the correct test of the milk 
CHAPTER III.

\section{THE BABCOCK TEST.}

34. The Babcock test is based on the fact that strong sulfuric acid will dissolve all non-fatty solid constitu-

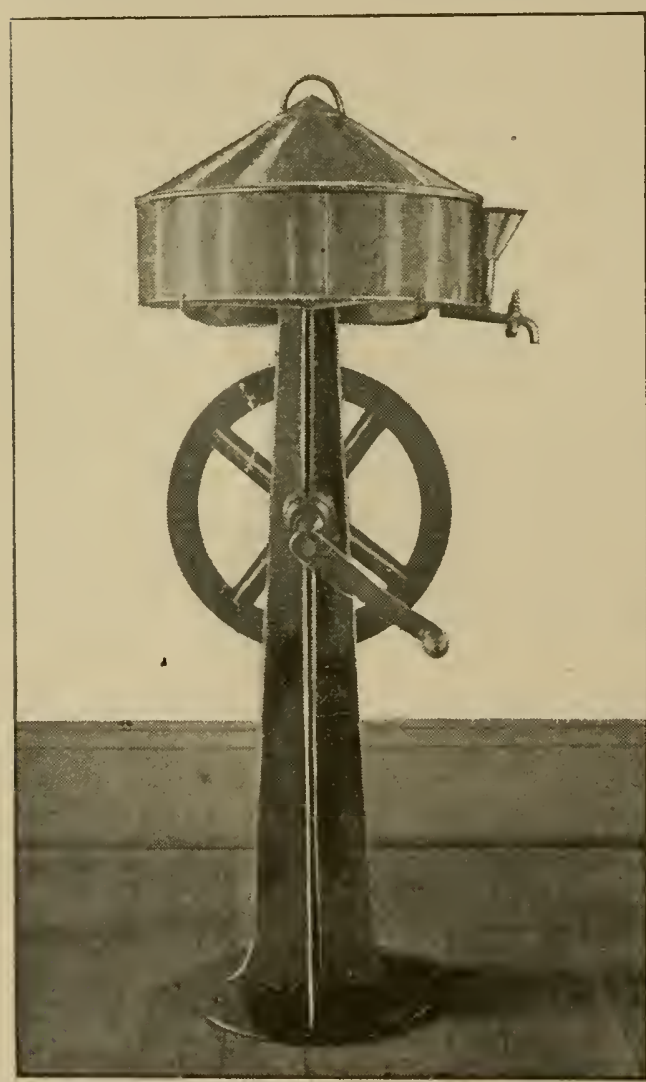

FIG. 4. The first Babcock tester made. ents of milk and other dairy products, and thus enable the fat to separate on standing. To effect a speedy and complete separation of the fat, the bottles holding the mixture of milk and acid are placed in a centrifugal mach in e, a so-called tester, and whirled for four minutes; hot water is then added so as to bring the liquid fat into the graduated neck of the test bottles, and after a repeated whirling, the length of the column of fat is read off, showing the per cent. of fat contained in the sample tested. 
Sulfuric acid is preferable to other mineral acids for the purpose mentioned, on account of its affinity to water; when mixed with milk, the mixture heats greatly, thus keeping the fat liquid without the application of artificial heat and rendering possible a distinct reading of the column of fat brought into the neck of the test bottles.

So far as is known, any kind of milk can be tested by the Babcock test. Breed, period of lactation, quality or age of the milk are of no importance in using this method, so long as a fair sample of milk can be secured. In cases of samples of milk or other dairy products rich in solids it requires a little more effort to obtain a thorough mixture with the acid than with dairy products low in solids, like skim milk or whey, which may be readily mixed with the acid.

\section{A.-Directions for Making the Test.}

35. The various steps in the manipulation of the Babcock test are discussed in the following pages; attention is drawn to the difficulties which the beginner and others may encounter in the use of the test, and the necessary precautions to be observed in order to obtain accurate and satisfactory results are explained in detail. The effort has been to treat the subject exhaustively and from a practical point of view, so that persons as yet unfamiliar with the test may turn to the pages of this book for help in difficulties which they may meet in their work in this line.

36. Sampling. The sample to be tested is first mixed by pouring the milk from one vessel to another two or 
three times, so that every portion thereof will contain a uniform amount of butter fat (29). The measuring pipette (fig. 6), which has a capacity of 17.6 cubic centimeters, ${ }^{1}$ is filled with

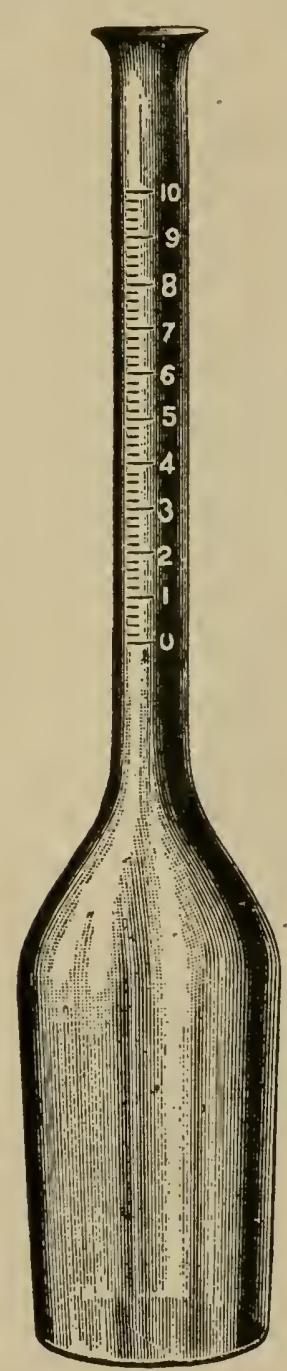

FIG. 5. Babcock milk test bottle.

the milk immediately after the mixing is completed, by sucking the milk into it until this rises a little above the mark around the stem of the pipette; the forefinger is then quickly placed over the end of the pipotte before the milk runs down below the mark. By slightly releasing the pressure of the finger on the end of the pipette, the milk is now allowed to run down until it just reaches the mark on the stem; the quantity of milk contained in the pipette will then, if this is correctly made, be exactly $17.6 \mathrm{cc}$. The finger should be dry in measuring out the milk so that the delivery of milk may be readily checked by gentle pressure on the upper end of the pipette.

The point of the pipette is now placed in the neck of a Babcock test bottle (fig. 5),

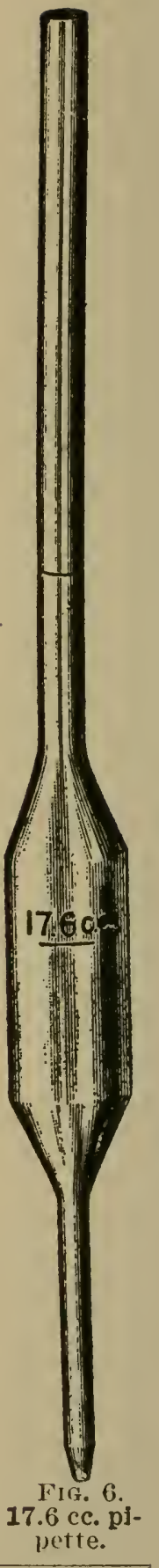

\footnotetext{
I See p. 45, foot note.
} 
and the milk is allowed to flow slowly down the inside of the neck. Care must be taken that none of the milk measured out is lost in this transfer. The portion of the milk remaining in the point of the pipette is blown into the test bottle.

The best and safest manner of holding the bottle and the pipette in this transfer is shown in fig. 7. Fig. 8 shows a position which should be avoided, since by holding the bottle in this way, there is

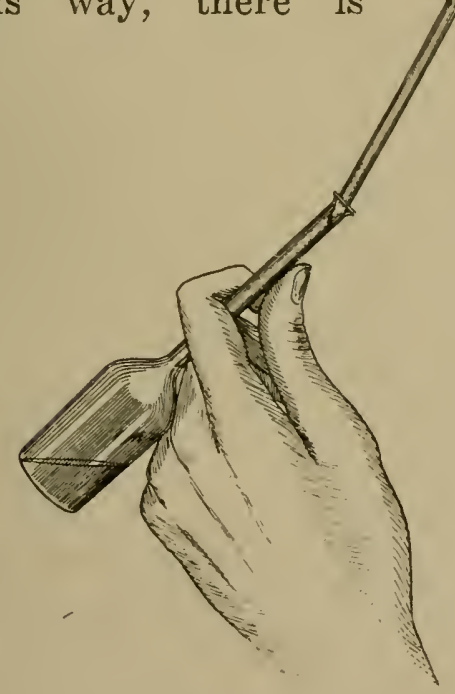

Vic. 7 . The right way of emptying pipette into test bottle.

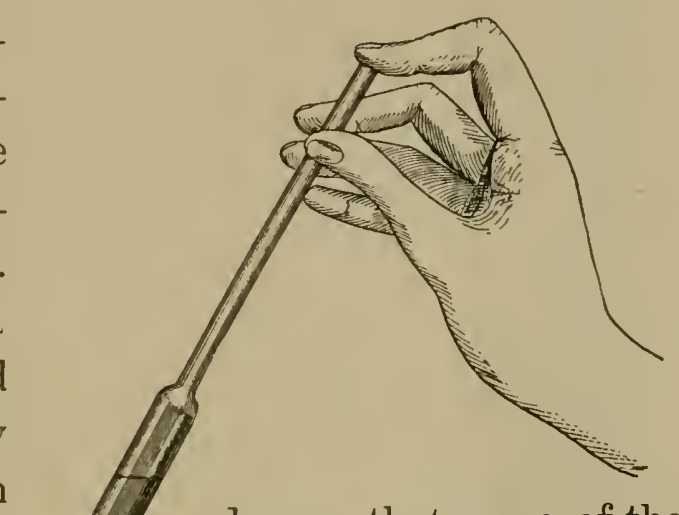

danger that some of the milk may completely fill the neck of the bottle, and as a result, flow over the top of the neck.

Pipettes, the lower part of which slip readily into the necks of the test bottles, may be emptied by lowering the pipette into the neck of the bottle till it rests on its rim, when the milk is allowed to run into the test bottle. 37. Adding acid. The acid cylinder (fig. 9) holding 17.5 cc., is filled to the mark with sulfuric acid of 
a specific gravity of 1.82-1.83. This amount of acid is carefully poured into the test bottle containing the milk. In adding the acid, the test bottle is conveniently held at an angle (see fig. 7), so that the acid will run down the wall of the bottle and not run in a small stream into the center of the milk, the bottle being slowly turned around and the neck thus cleared of adhering milk. By pouring the acid into the middle of the test bottle, there is also a danger of completely filling this with acid, in which case the plug of acid formed will be pushed over the edge of the neck by the expansion of the air in the bottle, and may be spilled on the hands of the operator.

The milk and the acid in the test bottle should be in two distinct layers, without much of a black band of partially mixed liquids between them. Such a dark layer is of-

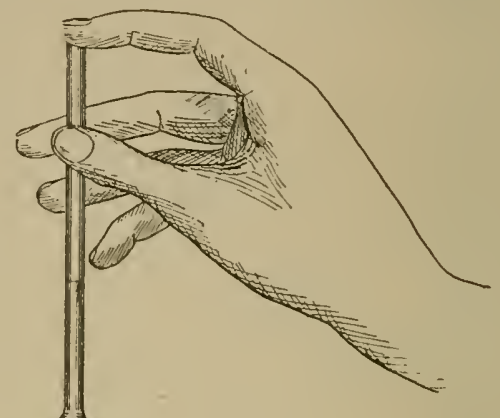
ten followed by an indistinct separation of the fat in the final reading. The cause of this may be that a partial mixture of acid and milk before the acid is diluted 
with the water of the milk will bring about too strong an action of the acid on this small portion of the milk, and thus char the fat contained therein. The appearance of black flocculent matter in or below the columin of fat which generally results, in either case renders a correct measurement difficult and at times even impossible; if the black specks occur in the fat column itself, the readings are apt to be too high; if below it, the difficulty comes in deciding where the column of fat begins.

38. Mixing milk and acid. After adding the acid, this is carefully mixed with the millk by giving the test bottle a rotary motion. In doing this, care should be taken that the liquid is not shaken into

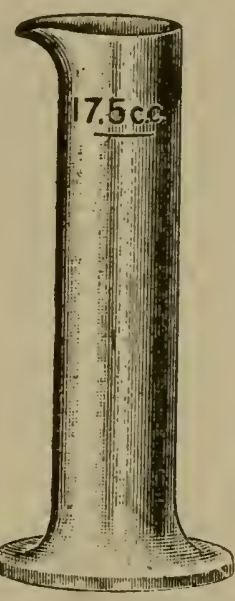

Frg. $9.17 .5 \mathrm{cc}$. acid cylinder. the neck of the test bottle. When once begur, the mixing should be continued until completed; a partial and interrupted mixing of the liquids will often cause more or less black material to separate with the fat when the test is finished. Clots of curd which separate at first by the action of the acid on the milk, must be entirely dissolved by continued and careful shaking of the bottle. Beginners sometimes fail to mix thoroughly the milk and the acid in the test bottle. As the acid is much heavier than the milk a thin layer of it is apt to be left unnoticed at the bottom of the bottle, unless this is vigorously shaken toward the end of the operation.

The mixture becomes hot by the action of the acid on the water in the milk and turns dark colored, owing to the effect of the strong sulfuric acid on the nitrogenous constituents and the sugar in the milk. 
Colostrum milk or milk from fresh cows will form a violet colored mixture with the acid, due to the action of the latter on the albumen present in such milk in considerable quantities (23).

When milk samples are preserved by means of potassium bichromate (190), and so much of this material has been added that the milk has a dark yellow or reddish color, the mixture of milk and acid will turn greenish black, and a complete solution is rendered extremely difficult on account of the toughening effect of the bichromate on the precipitated casein. The difficulty is still more pronounced with milk preserved with formaldehyd.

39. Whirling bottles. After the milk and the acid have been completely mixed, the test bottle is at once placed in the centrifugal machine or tester and whirled for four or five minutes at a speed of 600 to 1200 revolutions per minute, according to the diameter of the tester (66). It is not absolutely necessary to whirl the test bottles in the centrifuge as soon as the milk and the acid are mixed, although this method of procedure is much to be preferred; they may be left in this condition for any reasonable length of time ( 24 hours, if necessary) without the test being spoiled. If left until the mixture becomes cold, the bottles should, however, be placed in warm water (of about $160^{\circ} \mathrm{F}$.) for about fifteen minutes before whirling.

Four minutes at full speed is sufficient for the first whirling of the test bottles in the centrifuge; this will bring all fat to the surface of the liquid in the bottle. 
40. Adding water. Hot water is now added by means of a pipette or some special device (10 in fig. 58), until the bottles are filled to near the scale on the neck (80). The bottles are whirled again at full speed for one minute, and hot water added a second time, until the lower part of the column of fat comes within the scale on the neck of the test bottle, preferably to the 1 or 2 per cent. mark, so as to allow for the sinking of the column of fat, due to the gradual cooling of the contents of the bottle. By dropping the water directly on the fat in the second filling, the column of fat will be washed free from light flocculent matter, which might otherwise be

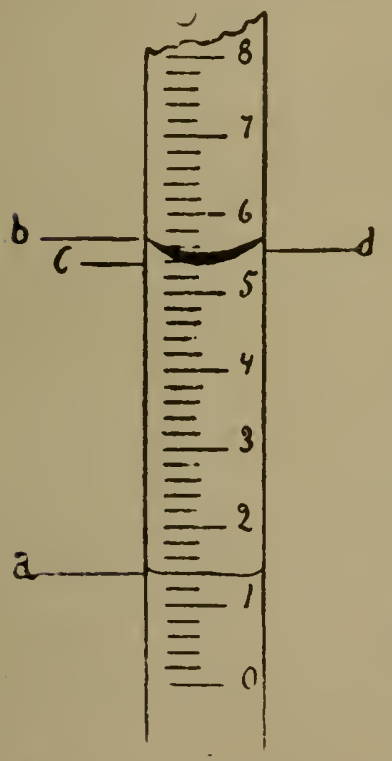

FIG. 10. Measuring the column of fat in a Babcock test bottle. entangled therein and render the reading uncertain or too high. A final whirling for one or two minutes completes the separation of the fat.

4r. Measuring the fat. The amount of fat in the neck of the bottle is measured by the scale or graduations on the neck. Each division of the scale represents two-tenths of one per cent. of fat, and the space filled by the fat shows the per cent. of butter fat contained in the sample tested.

The fat is measured from the lower line of separation between the fat and the water, to the top of the fat column, at the point $b$, shown in the figure, the reading being thus taken from $a$ to $b$, and not to $c$ or to $d$. Comparative 
gravimetric analyses have shown that the readings obtained in this manner give correct results. While the lower line of the fat column is nearly straight, the upper one is curved, and errors in the reading are therefore easily made, unless the preceding rule is observed.

The fat obtained should form a clear yellowish liquid distinctly separated from the acid solution beneath it. There should be no black or white sediment in or below the column of fat, and no bubbles or foam on its surface. The bottles must be kept warm until the readings are made, so that the column of fat will have a sharply defined upper and lower meniscus. When the testing is done in a cold room, it is a good plan to place the bottles in a pail with water of $140^{\circ} \mathrm{F}$. before readings are made. The readings should always be made when the fat has a temperature of about $140^{\circ}$ F.; too low results will be obtained if the fat is allowed to cool below $120^{\circ} \mathrm{F}$., and too high if readings are taken above $150^{\circ}$. The fat separated in the Babcock test solidifies at about $100^{\circ} \mathrm{F}$. If the fat is partly solidified, it is impossible to make an accurate reading. ${ }^{1}$

42. Readings of tests of milk made in steam turbine testers with tightly closed covers which prevent the free escape of exhaust steam (71), will come .2 to .3 per cent.

\footnotetext{
1 The effect of differences in the temperature of the fat on the readings obtained will be seen from the following: If 110 and $150^{\circ} \mathrm{F}$. be taken as the extreme temperatures at which readings can be made, this difference of $40^{\circ} \mathrm{F}$. $\left(22.3^{\circ} \mathrm{C}\right.$.) would make a difference in the volume of the fat column obtained in the case of 10 per cent. milk of $.00064 \times 2 \times 22.3=.028544$ cc., or .14 per cent., .00064 being the expansion coefficient of pure butter fat per degree Centigrade between 50 and $100^{\circ}$ C. (Zune, Analyse des Beurres, I, 87), and 2, the volume of the fat in cc. contained in $17.6 \mathrm{cc}$. of 10 per cent. milk. On 5 per cent. milk this extreme difference would therefore be about .07, or nearly one-tenth, of one per cent.
} 
too high if the temperature of the fat is allowed to rise to that of the exhaust steam during the process of whirling. In such cases the test bottles must be allowed to cool to about $140^{\circ}$, by placing them in water of this temperature for a few minutes, before readings are taken. ${ }^{1}$

A pair of dividers will be found convenient for measuring the fat, and the liability of error in reading is decreased by their use. The points of the dividers are placed at the upper and lower limits of the fat column (from $a$ to $b$ in fig. 10). The dividers are now lowered, one point being placed at the zero mark of the scale, and the mark at which the other point touches the scale will show the per cent. of fat in the sample tested. The dividers must be tight in the joint to be of use for this purpose.

\section{B.-Discussion of the Detalls of the BABCOCK TEST.}

43. The main points to be observed as to apparatus and testing materials in order to obtain correct and satisfactory results by this test will now be considered, and such suggestions and help offered as have been found needful from past experience with a great variety of samples of milk, apparatus, and accessories.

\section{1.-GLassware.}

44. Test bottles. The test bottles should have a capacity of about 50 cc., or less than two ounces; they should be made of well-annealed glass that will stand

${ }^{1}$ See Wis. Expt. Sta. rep., XVII, p. 76. 
sudden changes of temperature without breaking, and should be sufficiently heavy to withstand the maximum centrifugal force to which they are likely to be subjected in making tests. This force may, on the average, be not far from $30.65 \mathrm{lbs}$. (see 66), which is the pressure exerted in whirling the bottles filled with milk and acid in a centrifugal machine of 18 inches diameter at a speed of 800 revolutions per minute.

Special forms of test bottles used in testing cream and skim milk are described under the heads of cream and skim-milk testing $(89,90,99)$.

When 17.6 cc., or 18 grams of milk (48), are measured into the Babcock test bottle, the scale on the neck of the bottles will show directly the per cent. of fat found in the milk. The scale is graduated from 0 to 10 per cent. 10 per cent. of 18 grams is 1.8 grams. As the specific gravity of pure butter fat (i. e., its weight compared with that of an equal volume of pure water) at the temperature at which the readings are made (about $140^{\circ} \mathrm{F}$.), is 0.9 , then 1.8 grams of fat will occupy a volume of $\frac{1.8}{.9}=2$ cubic centimeters. The space between the 0 and 10 per cent. marks on the necks of the test bottles must therefore hold exactly 2 cubic centimeters. The scale is divided into 10 equal parts, each part representing one per cent., and each of these is again sub-divided into five equal parts. Each one of the latter divisions therefore represents two-tenths of one per cent. of fat when 17.6 cc. of milk is measured out. The small divisions are sufficiently far apart in most Babcock test bottles to make possible the estimation of onetenth, or even five-hundredths, of one per cent. of fat in the samples tested. 
As the necks of Babcock test bottles vary in diameter, each separate bottle must be calibrated by the manufacturers; the length of the scale is not,' for the reasons given, apt to be the same in different bottles. ${ }^{1}$

If the figures and lines of the scale become indistinct by use, the black color may be restored by rubbing a soft pencil over the scale, or by the use of a piece of burnt cork after the scale has been rubbed with a little tallow. On wiping the neck with a cloth or a piece of paper the black color will show in the etchings of the glass, making these plainly visible.

45. Marking test bottles. Test bottles can now be bought with a small band or portion of their neck or body ground or "frosted," for numbering the bottles with a lead pencil. Bottles without this ground label can be roughened at any convenient spot by using a wet fine file to roughen the smooth surface of the glass. There is this objection to the latter method that unless carefully done, it is apt to weaken the bottles so that they will easily break, and to both methods, that the lead pencil marks made on such ground labels may be effaced during the test if the bottles are not carefully handled. Small strips of tin or copper with a number stamped therecn are sometimes attached as a collar around the necks of the bottles. They are, however, easily lcst, especially when the top of the bottle is

${ }^{1}$ A flat-bore test bottle and one with a brass collar and screw used for opening and closing a small hole in the neck of the test bottle have been placed on the market by an Eastern manufacturer. These have been tried by us, and were not found to possess any particular advantage over the round-neck bottles; in fact, are more subject to errors of calibration. Bottles with figures at the left of the scale are also made and can be obtained if desired. 
slightly broken, or at any rate, are soon corroded so that the numbers can only be seen with difficulty.

The best and most permanent label for test bottles is made by scratching a number with a marking diamond on the glass directly above the scale on the neck of the bottles or by grinding a number on the bottle itself. In ordering an outfit, or test bottles alone, the operator may specify that the bottles

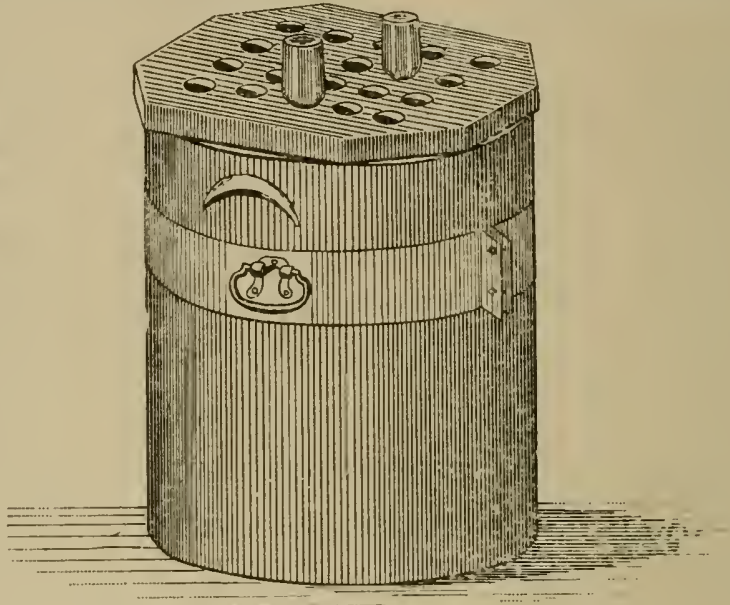

FiG. 12. Waste-acid jar. are to be marked 1 to 24 , or as many as are bought, and the dealer may then put the numbers on with a marking diamond.

A careful record should be kept of the number of the bottle into which each particular sample of milk is measured. Mistakes are often made when the operator trusts to his memory for locating the different bottles in which tests are made at the same time.

46. Cleaning test bottles. The fat in the neck of the test bottles must be liquid when these are cleaned. In emptying the acid the bottle should be shaken in order to remove the white residue of sulfate of lime, etc., from the bottom; if the acid is allowed to drain out of the bottle without shaking it, this residue will be found to stick very tenaciously to the bottom of the bottle in the subsequent cleaning with water. 
A convenient method of emptying test bottles is shown in the illustraticn (fig. 12). After reading the fat column, the bottles are placed neck down, in the half-inch holes of the board cover of a five-gallon stoneware jar.

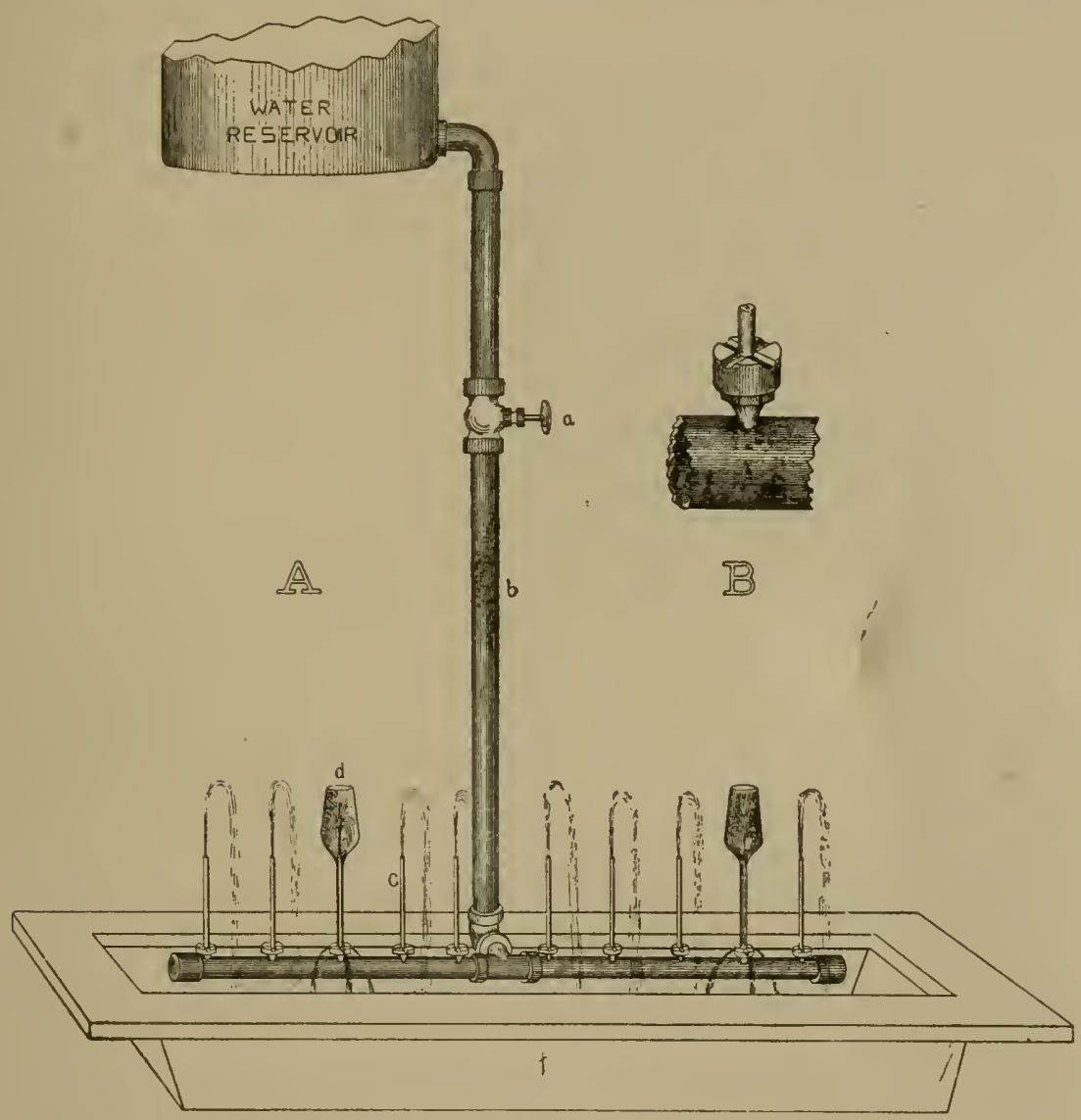

FIG. 13. Apparatus for cleaning test bottles. A, apparatus in position; the water flows from the reservoir through the iron pipe $b$ into the inverted test bottle $d$ through the brass tube $c$, screwed into the iron pipe. $B$ shows construction of the rubber support on which the test bottles rest; $f$, sink.

An occasional shaking while the liquid is running from the bottles will rinse off the preciptate of sulfate of lime. A thorough rinsing with boiling hot water is 
generally sufficient to remove all grease and dirt, as well as acid solution from the inside of the bottles. The apparatus shown in fig. 13 will be found convenient for this purpose. After the bottles have been rinsed a second time, they may be placed in an inverted position to drain, on a galvanized iron rack, as shown in fig. 14, where they are kept until needed. The outside

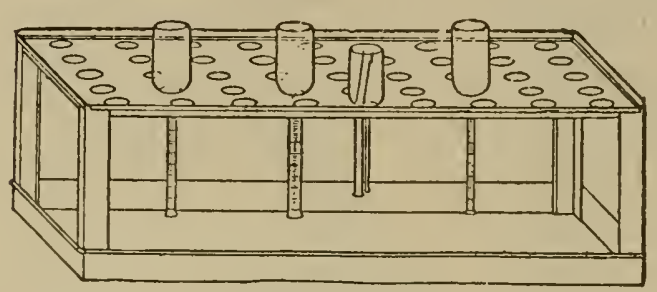

FIG. 14. Draining rack for test bottles. of the bottles should occasionally be wiped clean and dry.

47. The amount of unseen fat that clings to test bottles used for testing milk or cream, is generally not sufficient to be noticed in test. ing whole rnilk, but it plays an important part in test. ing samples of separator skim milk. It may be readily brought to light by making a blank test with clean water in bottles used for testing ordinary milk, which have been cleaned by simply. draining the contents and rinsing once or twice with hot water; at the conclusion of the test the operator will generally find that a few drops of fat will collect in the neck of the bottles, sometimes enough to condemn a separator.

Boiling hot water will generally clean the grease from glassware for a time, but all test bottles should, in addition, be given an occasional bath in some weak alkali or other grease-dissolving solution. Persons doing considerable milk testing will find it of advantage to provide themselves with a small copper tank, fig. 15, which can be filled with a weak alkali-solution. After 
having been rinsed with hot water, the test bottles are placed in the hot solution in the tank, where they may be left completely covered with the liquid. If the tank is provided with a small faucet at the bottom, the liquid can be drawn off when the test bottles are wanted. A tablespoonful of some cleaning powder to about two gallons of water will make a very satisfactory solution; sal soda, Gold Dust, Lewis' lye or Babbitt's potash are very efficient for this purpose. The cleansing properties of solutions of any of these substances are increased by warming the liquid. The

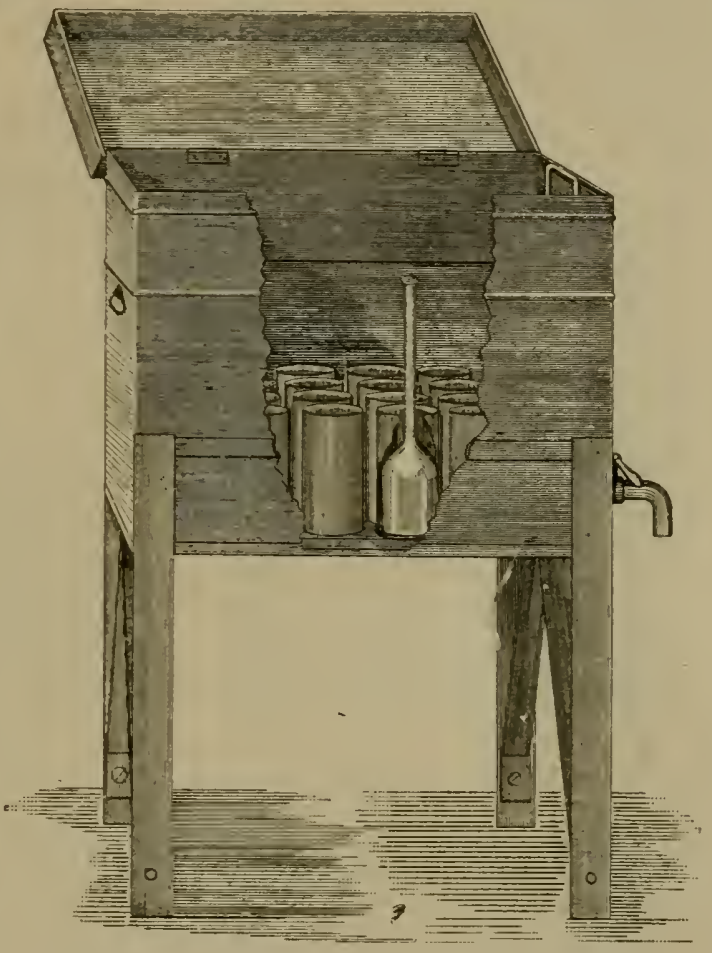
test bottles must be rinsed twice with hot water after they are taken from this bath.

An excellent cleaning solution that can be used for a long time, may be made of one-half pound bichromate of potash to one gallon of sulfuric acid. ${ }^{1}$

47a. An arrangement for cleaning a number of test bottles at the same time is shown in fig. 16. ${ }^{2}$ III shows

${ }_{1}^{1}$ Michels, Am. Cheescmaker, Jan. 1903.

2 Wisconsin experiment station, bull. 129. 
the frame in which the bottles are placed, one in each socket; the metal plate $\mathrm{E}$ is put over the necks of the bottles which pass through the holes in it up to the shoulder of the bottles. The pins F, F, are then pushed through holes in the rods D-D, and the plate and bottles thus firmly held in the crate. When secured in

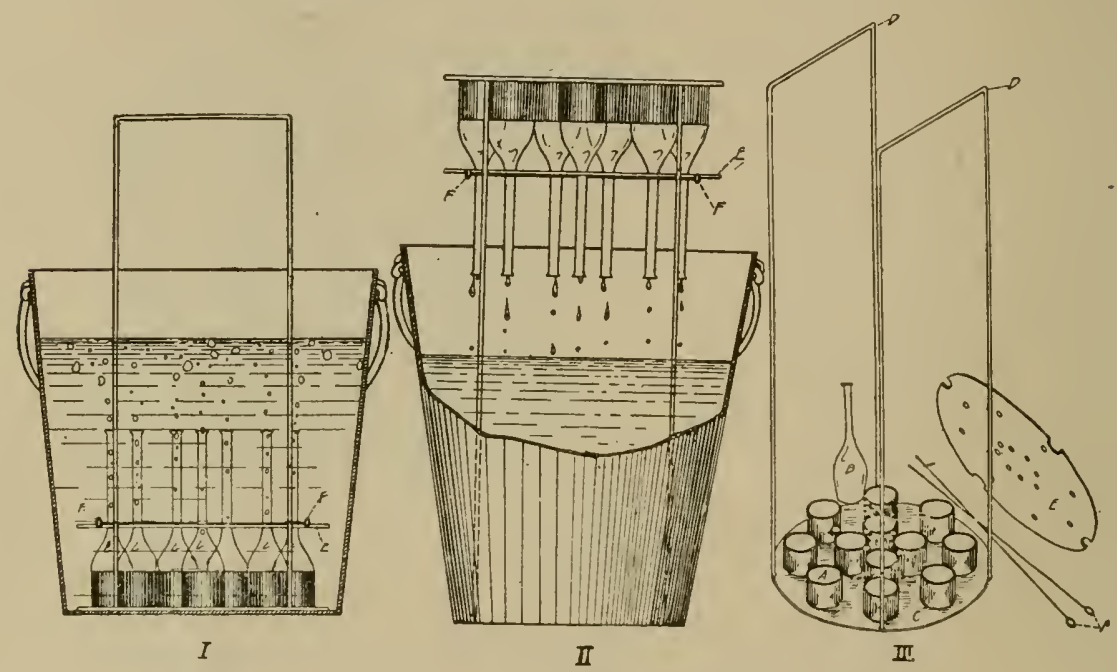

Fig. 16. A convenient device for cleaning test bottles.

this way, the frame full of bottles may be placed in a pail or tank of hot water as in I. They will soon fill with water and the time of filling the bottles one at a time thus saved. When ready to empty the bottles, the frame is reversed and placed in the position shown in II.

One or two rinsings in boiling hot water is usually sufficient to effectually clean the bottles, but when they have been allowed to get greasy they can be dipped into a pail of hot dilute lye; this will saponify the grease and after one or two rinsings in clean hot water the bottles will be bright and clean. 
The black stains that sometimes stick to the inside of test bottles after prolonged use, can be removed with a little muriatic acid, or by means of a small stiff brush.

48. Pipette. The difference in the weights of various samples of normal milk generally falls within comparatively narrow limits; if a given volume of water weighs one pound, the same volume of the usual grades of normal milk will weigh from 1.029 to 1.033 pounds, or on the average, $1.03 \mathrm{lbs}$. 18 grams of water measures 18 cc. ${ }^{1}$; 18 grams of mill will therefore take up a smaller volume than 18 cc., viz., 18 divided by 1.03, which is very nearly 17.5. This is the quantity of milk taken in the Babcock test. A certain amount of milk will adhere to the walls of the pipette when it is emptied, and this thin film has been found to weigh about one-tenth of a gram; consequently 17.6 cc. has been adopted as the capacity of the pipette used for delivering 18 grams of milk.
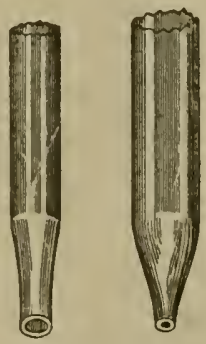

For convenience in measuring the milk, the shape of the pipette is of importance. The mark on the stem should be two inches or more from the upper end of the pipFIG. 17. Pipette pointsA, proper construction: $\mathrm{B}$, undesirable construction. ette. The lower part should be small enough to fit loosely into the neck of the test bottle, and not contracted to a fine hole at the point; the point should be

\footnotetext{
1 Cubic centimeters (abbreviated: cc.) are the standard used for measuring volume in the metric system, similar to the quart or pint measure in our oldinary system of measures. One quart is equal to a little less than 1,000 cubic centimeters ( 1 liter). In the same way, grams represent weight, like pounds and ounces. One cc. of water at $4^{\circ}$ Centigrade weighs $1^{\circ}$ gram; 1,000 grams $(=1$ kilogram $)$ are equal to 2.2 lbs. Avoirdup. (See Appendix for Comparisons of metric and customary weights and measures.)
} 
large enough to allow a quick emptying of the pipette (fig. 17) and not so large that it is difficult to use it. An opening of about $1 / 8$ in. diameter will be found satisfactory.

49. Fool pipettes. Soon after the Babcock test began to be generally used at creameries as a basis of payment for the milk, a creamery supply house put on the market a 20 cc. milk-measuring pipette, which was claimed to show the exact butter value of milk, instead of its content of butter fat, as is the case in using the nrdinary 17.6 cc. pipette. A 20 cc. pipette will deliver $2.4 \mathrm{ce}$. more milk than a $17.6 \mathrm{cc}$. pipette, (or $13.6 \mathrm{per}$ cent. more), and the results obtained by using these pipettes will, therefore, be about 13.6 per cent. too high. In considering the subject of Overrun (214) it is noted that the excess of butter yield over the amount of fat contained in a certain quantity of milk will range from about 10 to 16 per cent., or on the average, about 12 per cent. $20 \mathrm{cc}$. pipettes may, therefore, give approximately the yield of butter obtained from a quantity of milk, but as will be seen, this yield is variable, according to the skill of the butter maker and to conditions beyond his control; it cannot therefore be used as a standard in the same manner as the fat content of milk. Similar 22 cc. pipettes were also sent out. These pipettes created a great deal of confusion during the short time they were on the market, and were popularly termed "fool" pipettes. It is not known that such pipettes have been sold of late years.

A Wisconsin law makes it a misdemeanor to use in that state other than $17.6 \mathrm{cc}$. pipettes for measuring milk where this is paid for by the Babcock test. ${ }^{1}$

50. Acid measures. A 17.5 cc. glass cylinder (fig. 9) for measuring the acid is generally included in the outfit, when a Babcock tester is bought. This cylinder answers every purpose if only occasional tests are made; the acid is poured into the cylinder from the acid bottle as needed, or a quantity of acid sufficient for the number of test bottles to be whirled at a time, is poured

\footnotetext{
${ }^{1}$ Laws of 1903 , chapter 43.
} 
into a small glass beaker provided with a lip, or into a small porcelain pitcher; these may be more easily handled than the heavy acid bottle or jug, and the acid measure is then filled from such a vessel.

Where a considerable number of tests are made regularly, the acid can be measured into the test bottles faster and with less danger of spilling, by using some one of the many devices proposed for this purpose. There is some objection to nearly all of these appliances, automatic pipettes, burettes, etc., although they will often give good satisfaction for a time while new. Sulfuric acid is very corrosive, and operators, as a rule, take but poor care of such apparatus, so that it is a very difficult matter to design a form which will remain in good working order for a long time. Automatic pipettes attached to acid bottles or reservoirs, to prove satisfactory, must be made entirely of glass, and strong, of simple construction, tightly closed and quickly operated.

\section{The Swedish acid bot-} tle $^{1}$ answers these requirements better than any other device

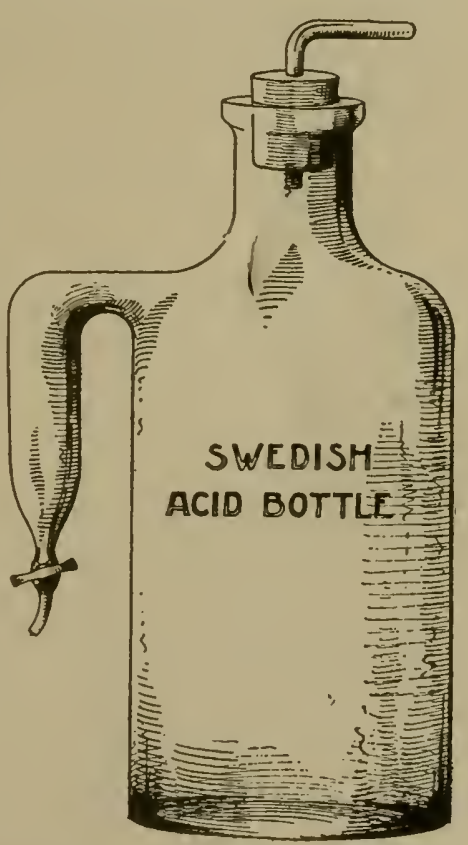
FIG. 18. Swedish acid-bottle; the side tube is made to hold $17.5 \mathrm{cc}$. of acid. known to the writers at the present time. Its use is easily understood (see fig. 18); it gives good satisfac-

${ }^{1}$ Now generally sold and known as the Combined Acid Bottle. 
tion if the hole in the glass stop-cock through which the acid passes has a diameter of at least one-eighth of an inch, as is generally the case. We have used or inspected some half a dozen other devices placed on the market by various dealers for delivering the acid, but cannot recommend them for use in factories or outside of chemical laboratories.

52. Instead of measuring out the acid, Bartlett ${ }^{1}$ has suggested adding $20 \mathrm{cc}$. directly to the milk in the test bottles, till the mixture rises to a mark on the body of the bottle at the point where this will hold $37.5 \mathrm{cc}$., i. e., the total volume of milk and acid (83). This method of adding the acid is in the line of simplicity, but has not become generally adopted. If the method is used, the marks should be put on by the manufacturers, as the operator in attempting to do so will be apt to weaken or break the bottles.

\section{Calibration of Glassware.}

53. Test bottles. The Babcock milk test bottles are so constructed that the scale of graduation on the neck measures a volume of 2 cubic centimeters, between the zero and the 10 per cent. marks (44). The standards for test bottles and other Babcock glassware adopted by the Association of Official Agricultural Chemists of America are given at the close of this book (306). It will be seen that the limit of error for test bottles is one of the smallest graduations on the scale, or .2 per cent. The correctness of the graduations may be easily ascertained by one of the following methods:

54. (A.) Calibration with water. This may be done by means of a delicate pipette or burette, or by weighing the water that the graduated portion of the neck will hold. 
a, Measuring the water. Fill the test bottle with water to the zero mark on the scale; remove any surplus water and dry the inside of the neck with a piece of filter paper or clean blotting paper; then measure into the bottle 2 cc. of water from an accurate pipette or burette, divided to one-twentieth of a centimeter. If the graduation is correct, 2 ec. will fill the neck exactly to the 10 per cent. mark of the scale.

b, Weighing the water. Fill the bottle with water to the zero mark of the scale and remove any surplus water in the neck, as before. Weigh the bottle with the water contained therein. Now fill the neck with water to the 10 per cent. mark, and weigh again. The difference between these weights should be 2 grams.

In all cases where calibrations are to be made, the test bottles, or other glassware to be calibrated, must be thoroughly cleaned beforehand with strong sulfuric acid or soda lye, and washed repeatedly with pure water, and dried. Glassware is not clean unless water will run freely over its surface, without leaving any adhering drops.

55. (B). The Trowbridge method of calibration. ${ }^{1}$ An extremely simple and accurate method of calibrating test bottles has been proposed by Mr. O. A. Trowbridge of Columbus, Wis. The capacity of the graduated portion of the neck of a milk test bottle is measured with a piece of metal which is carefully filed to such a size that it will displace exactly two cubic centimeters of water. He used a thirty-penny wire nail, eutting off the liead

\footnotetext{
${ }^{1}$ Hoard's Dairyman. March S, 1901, by DeWitt Goodrich.
} 
of the nail and attaching to it a short piece of fine wire. Manufacturers have improved on this rather crude de-

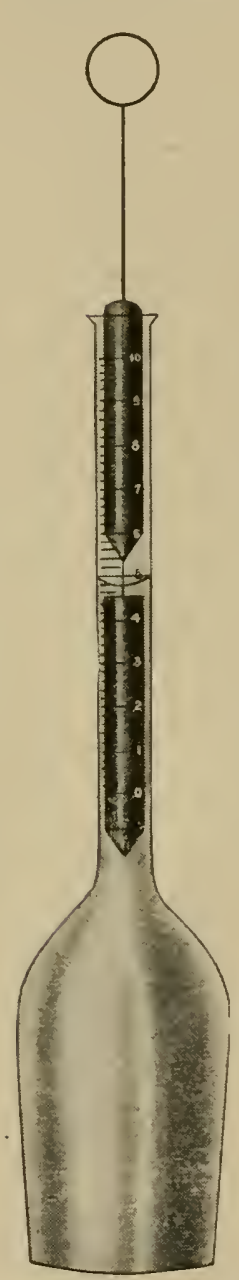
vice and standard measures for calibrating test bottles can now be bonght of dairy supply houses (see fig. 19).

When a test bottle is to be calibrated by this standard measure, it is filled with water to the zero mark on the neck of the bottle. The water adhering to the neck is carefully removed with a strip of blotting paper, and the measure is then lowered into the test bottle, as shown in the illustration. If the water rises from 0 to 10 on the neck when the upper point of the measure is submerged in the water, the scale is correct. If greater variations than .2 per cent. occur, the bottle should be rejected.

The figure shows one of these calibrators made in two sections, so that the accuracy of the 5 per cent., as well as the 10 per cent. mark on the scale may be ascertained.

56. The standard measure. In the place of an iron nail, as originally proposed, a Fic. 19. The piece of metal or glass rod may be advanibrator. tageously used as a standard measure. The standardization of this measure is most conveniently done by weighing. Since the specific gravities of iron, copper, brass, and glass are 7.2, 8.7, 8.5, and about 2.7, respectively, pieces of these materials replacing 2 cc. of 
a liquid, will weigh 14., 17.4, 17.0 and 5.4 grams, for iron, copper, brass and glass in the order given.

A measure of the right weight may be suspended by a very fine copper or platinum wire (melted into the glass rod if this material be chosen), and is used directly for calibrating test bottles as described above. Before a measure so made is used as a standard, its accuracy should be determined by weighing the amount of water of a temperature of $20^{\circ} \mathrm{C}$., which it replaces. The specific gravity of glass especially, varies somewhat according to its composition, so that a standardization of a measure by weight alone cannot be depended upon to always give correct results.

In submerging the measure in the test bottle to be calibrated, care must be taken that all air bubbles are removed before the position of the meniscus of the water is noted; if a metal standard measure is used, it must be kept free from rust or tarnish.

57. (C.) Calibration with mercury. 27.10 grams of metallic mercury are weighed into the perfectly clean and dry test bottle. Since the specific gravity of mercury is 13.55, double this quantity will occupy a volume of exactly 2 cubic centimeters (48). The neck of the test bottle is then closed with a small, smooth and soft cork, or a wad of absorbent cotton, cut off square at one end, the stopper being pressed down to the first line of the graduation. The bottle is now inverted so that the mercury will run into its neck. If the total space included between the 0 and 10 marks is just filled by the two cubic centimeters of mercury, the graduation is correct.

The mercury may be conveniently transferred from one test bottle to another, by means of a thin rubber tube which is slipped over the end of the necks of both bottles, and one weighing of mercury will thus suffice for a number of calibrations. In transferring the mercury, care must be taken that none of it 
is lost, and that small drops of mercury are not left sticking to the walls of the bottle emptied. A sharp tap on the bottle with a lead pencil will help to remove minute drops of mercury from the inside. Unless the bottles to be calibrated are perfectly clean and dry, it is impossible to transfer all the mercury from one bottle to another.

After several calibrations have been made, the mercury should be weighed again in order to make certain that none has been lost by the various manipulations. Scales similar to those shown under (91) are sufficiently delicate for making these weighings.

58. Test bottles may also be calibrated with mercury by weighing the bottles filled with mercury to the zero mark, and again when filled to the 10 mark. This is the official method for testing bottles adopted by the Association of Official Agricultural Chemists (see 306).

59. Cleaning mercury. Even with the best of care, mercury used for calibration of glassware will gradually become dirty, so that it will not flow freely over a clean surface of glass. It may be cleaned from mechanical impurities, dust, grease, water, etc., by filtration through heavy filter paper. This is folded in the usual way, placed in an ordinary glass funnel and its point perforated with a couple of pin holes. The mercury will pass through in fine streams, leaving the impurities on the filter paper. Mercury may be freed from foreign metals, zinc, lead, etc., sometimes noticed as a grayish, thin film on its surface, by leaving it in contact with common nitric acid for a number of hours; the mercury is best placed in a shallow porcelain or graniteware dish and the nitric acid poured over it, the dish being covered to keep out dust. The acid solution is then carefully poured off and the mercury washed with water; the latter is in turn poured off, and the last traces of water absorbed by means of clean, heavy filter paper.

The mercury to be used for calibration of glassware should be kept in a strong bottle, closed by an ordinary stopper. In handling mercury, eare must be taken not to spill any portion of it; finger-rings should be removed when calibrations with mercury are to be made.

Mercury forms the most satisfactory and accurate material for calibration of test bottles, on account of its heavy weight and the ease with which it may be manipulated. Equally correct 
results may, however, with proper care be obtained by using water for the calibration.

60. Intermediate divisions. The space between 0 and 10 on the scale of the Babcock test bottle is divided into 50 divisions, each five of which, as previously shown, represent 1 per cent. (44). Since these intermediate divisions are generally made with a dividing machine, they are as a rule correct, but it may happen that they have been inaccurately placed, although the space between 0 and 10 is correct. The accuracy of the intermediate divisions can be ascertained by sliding along the scale a strip of paper upon which has been marked the space occupied by one per cent., and comparing this space with those of each per cent. on the scale.

6r. Calibration of skim milk test bottlıs. The value of each division on the common double-necked skim milk bottles (99) is one-twentieth, or .05 of one per cent.; there are ten of these divisions in the whole scale which, therefore, measures .5 per cent. of fat. It requires very careful work to calibrate this scale and it is best done by weighing the amount of mercury which will just fill the space of .1 ce. between the first and the last divisions (53); the correct weight of this mercury is 1.355 grams.

62. Calibration of cream test botties. The cream bottles may be calibrated by any of the methods given for milk bottles. The neck of a cream test bottle that measures thirty per cent. fat will hold 6 cc., and 6 grams of water or 81.30 grams of mercury.

The Trowbridge method of calibrating milk test bottles will also be found convenient for cream bottles and 
the same standard measure used, the part of the scale from 0 to 10 being calibrated first, then that from 10 to 20, 20 to 30,30 to 40 per cent., etc., in the same way.

63. Pipette and acid cylinder. The pipette and the acid cylinder used in the Babcock test may be calibrated by any of the methods already given. Sufficiently accurate results are obtained by weighing the quantity of water which each of these pieces of apparatus will deliver, viz., 17.5 grams. The necessity of previous thorough cleaning of the glassware is evident from what has been said in the preceding. The pipette and the acid measure may be weighed empty and then again when filled to the mark with pure water, or the measureful of water may be emptied into a small weighed vessel, and this weighed a second time. In either case the weight of the water contained in the pipette or acid measure is obtained by difference. ${ }^{1}$

Calibrations of the acid cylinder are generally not called for, except as a laboratory exercise, since small variations in the amount of acid measured do not affect variations in the amount of acid measured out do not affect the accuracy of the test.

\section{2.-Centrifugal Machines.}

64. The capacity of the testing machine to be selected should be governed by the number of tests which are likely to be made at one time. For factory purposes a

${ }^{1}$ One cubic centimeter of distilled water weighs 1 gram, when weighed in a vacuum at the temperature of the maximum density of water $\left(4^{\circ}\right.$ C.) ; for the purpose of calibration of glassware used in the Babcock test, sufficiently accurate results are, however, obtained by weighing the water in the air and at a low room temperature $\left(60^{\circ} \mathrm{F}.\right)$ 
twenty-four or a thirty-two bottle tester is large enough, and to be preferred to a larger tester, even if a large number of samples are to be tested at a time. The operator can use his time more economically in running a machine of this size than one holding fifty or sixty bottles; the work of filling or cleaning the bottles and measuring the fat can be done while the tester is running if a double supply of bottles is at hand. Large testers require more power than smaller ones, and when sixty tests are made at a time, the fat column in many bottles will get cold, before the operator has time to read them, unless special precautions are taken for keeping the bottles warm.

65. The tester should be securely fastened to a solid foundation and set so that the revolving wheel is level. The latter must be carefully balanced in order that the tester may be run smoothly at full speed. A machine that trembles when in motion is neither satisfactory nor safe, and the results obtained are apt to be too low. High-standing machines are more likely to cause trouble in this respect than low machines, and should therefore be subjected to a severe test before they are accepted.

If all sockets are not filled with bottles when a test is to be made, the bottles must be placed diametrically opposite one another so that the machine will be balanced when run. The bearings should be kept clean and be oiled with as much care as the bearings of a cream separator.

The cover of the machine should always be kept closed while the bottles are whirled, and should not be 
removed until the machine stops; it should be tight fitting, since test bottles sometimes break while the machine is running at full speed, and every possible precaution should be taken to protect the operator from any danger from spilled acid or broken glass.

66. Speed required for the complete separacion of the fat. There is a definite relation between the diameter of the Babcock testers and the speed required for a perfect separation of the fat. In the preliminary work with the Babcock test the inventor found that with the machine used, the wheel of which had a diameter of eighteen inches, it was necessary to turn the crank so as to give the test bottles seven or eight hundred revolutions per minute, in order to obtain a maximum separation of fat; later work has shown that this speed is ample. Taking the higher figure as a standard, the centrifugal force to which the contents of the test bottles are subjected when supported on an eighteen-inch wheel and turned 800 revolutions per minute, can be calculated as follows:

The centrifugal force, $F$, acting on the bottles is expressed by the formula

$$
\mathrm{F}=\frac{\mathrm{w} \cdot \mathrm{v}^{2}}{32.2 \mathrm{r}}
$$

in which $\mathrm{w}=$ the weight of the bottle with contents, in pounds; $\nabla=$ the velocity, in feet per second, and $r=$ the radius of the wheel in feet.

When the wheel is turned 800 times a minute, a bottle supported on its rim will travel $2 \pi r \times \frac{800}{60} \times 2 \times 3.1415 \times \frac{9}{12} \times \frac{800}{600}=62.83$ feet per second. The weight of a bottle, with milk and acid, is about 3 ounces, or $\frac{3}{16}$ of a pound. Substituting these values for $\nabla$ and $w$, gives 


$$
\mathrm{F}=\frac{\frac{3}{165} \times 62.83^{2}}{32.2 \times{ }_{12}^{9}}=30.65 \mathrm{lbs} .
$$

The bottles are, therefore, under the conditions given, subjected to a pressure of about 30.65 pounds. In order to calculate the speed required for obtaining this force in case of machines of other diameters, the value of $\mathrm{v}$ in formula (I) is found from

$$
\begin{aligned}
& v=\sqrt{\frac{32.2 \mathrm{~F} \times \mathrm{r}}{\mathrm{w}}} \\
& \frac{32.2 \times 30.65 \mathrm{r}}{\frac{3}{16}}=V^{\prime} \overline{5264 \mathrm{r}}
\end{aligned}
$$

In this equation the values $\mathrm{r}=5,6,7,8,9,10,11,12$ inches are substituted in each case $\left(\frac{5}{2}, \frac{6}{12}, \frac{7}{1} \frac{7}{2}, \ldots . \frac{12}{12}\right)$, and the velocity in feet per second then found at which the bottles are whirled when placed in wheels of diameters 10 to 24 inches, and subjected in each case to a centrifugal force of $30.65 \mathrm{lbs}$. As the number of revolutions per minute $=\frac{60 v}{2 \pi \mathrm{r}}, v$ being as before the relocity in feet per second, and $r$ the radius of the wheel, the speed at which the wheel must be turned is found by substituting for $v$ the values obtained in the preceding calculations in case of wheels of different diameters. The results of these calculations are given in the following table:

$\begin{array}{ccc}\begin{array}{c}\text { Diameter } \\ \text { of wheel, } D .\end{array} & \begin{array}{c}\text { Velocity in feet } \\ \text { per second, } v\end{array} & \begin{array}{c}\text { Number of revolutions } \\ \text { of wheel per minute. }\end{array} \\ 10 & 46.84 & 1074 \\ 12 & 51.31 & 980 \\ 14 & 55.43 & 909 \\ 16 & 59.26 & 848 \\ 18 & 62.84 & 800 \\ 20 & 66.24 & 759 \\ 22 & 69.47 & 724 \\ 24 & 72.56 & 693\end{array}$

These figures show that a tester, e. g., 24 inches in diameter, will require less than 700 revolutions per minuate for a perfect separation of the fat in Babcock bottles, while a ten-inch tester must have a speed of nearly 1100 revolutions in order to obtain the same result. 
The speed at which testers of different diameters should be run to effect a complete separation has been calculated by Prof. C. L. Beach in the following manner. ${ }^{1}$ The same standard as before is taken, viz., 800 revolutions for an 18-inch tester (radius 9 inches); then if $x$ designate the radius of the tester and $y$ the speed required, we have

$$
\begin{aligned}
& x y^{2}=9 \times 800^{2}, \text { or } \\
& y=\sqrt{\frac{9 \times 800^{2}}{x}}
\end{aligned}
$$

The figures obtained by the use of this formula are similar to those given in the preceding table.

67. To find the number of turns of the handle corresponding to the number of revolutions made by the wheel, the handle is given one full turn, and the number of times which a certain point of the wheel revolves, is noted. If the wheel has a diameter of 20 inches and revolves 12 times for one turn of the handle, the latter should be turned $\frac{559}{1 \frac{5}{2}}=63$ times a minute (see table), or about once every second, in order to effect a maximum separation of fat. By counting the number of revolutions, watch in hand, and consulting the preceding table, the operator will soon note the speed which must be maintained in case of his particular machine. It is vitally important that the required speed be always kept up; if through carelessness, worn-out or dry bearings, low steam pressure, etc., the speed is slackened, the results obtained will be too low; it may be a few tenths, or even more than one per cent. Care as to this point is so much the more essential, as the results obtained by too slow whirling may seem to be all right, a clear

${ }^{1}$ Private communication. 
separation of fat being often obtained, even when the fat is not completely separated.

68. Ascertaining the necessary speed of testers. In buying a tester the operator should first of all satisfy himself at what speed the machine must be run to give correct results; the preceding table will serve as a guide on this point. He should measure out a dozen tests of the same sample of milk, and whirl half the number at the speed required for machines of the diameter of his tester. Whirl the other half at a somewhat higher speed. If the averages of the two sets of determinations are the same, within the probable error of the test (say, less than one-tenth of one per cent.), the first whirling was sufficient, as it is believed will generally be the case. If the second set of determinations come higher than the first set, the first whirling was too slow, and a new series of tests of the same sample of milk should be made to ascertain that the speed in the second set of determinations was sufficient.

This method will test not only the speed required with the particular machine at hand, but will also serve to indicate the correctness of the calibration of the bottles. A large number of tests of the same sample of milk made as directed (pouring the milk once or twice previously to taking out a pipetteful for each test) should not vary more than two-tenths of one per cent. at the outside, and in the hands of a skilled operator will generally come within one-tenth of one per cent. If greater discrepancies occur, the test bottles giving too high or too low results should be further examined, and calibrated according to the directions already given (53 et seq.). 
69. Hand testers. When only a few tests are made at a time, and at irregular intervals, as in case of dairy-

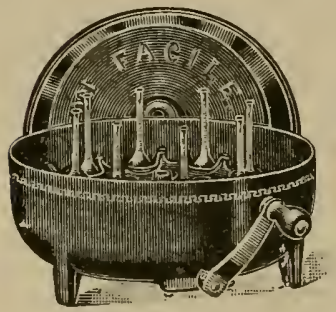

H'IG. 20. Type of Babcock hand testers. men who test single cows in their herds, a small hand tester answers every purpose. These may be had in sizes from two to twelve bottles. In selecting a particular make of tester the dairyman has the choice of a large number of different machines. Most of the first machines placed on the market for this purpose were so cheaply and poorly constructed as to prove very unsatisfactory after having been in use for a time. The competition between manufacturers of $\mathrm{d}$ a i ry supplies and the clamor of dairymen for something cheap, FIG. 21. Type of Babcock hand testers. fully accounted for this condition of affairs. This applies especially to the early machines made with belts or friction application of power. Hand testers made with cog-geared wheels can be depended on to give the necessary speed when run according to the manufacturers' directions; the earlier machines of this kind were very noisy, but at the present time the best machines on the market are of this type. These are provided with spiral cog-gearing and ball bearings, are strongly made and will run smoothly and with little noise (figs 
20 and 21); in cog-geared machines the bottles are always whirled at the speed which the number of turns made by the crank would indicate.

70. Power testers. For factory purposes, steam turbine machines (figs. 22-24) are most satisfactory when well made and well cared for. They should be provicled with a speed indicator and steam gauge, both for the purpose of knowing that sufficient speed is attained, and to prevent what may be serious accidents from a general smash-up, if the turbine "runs wild" by turning on too much steam. The revolving wheel of the tester should be made of wrought or malleable iron, or

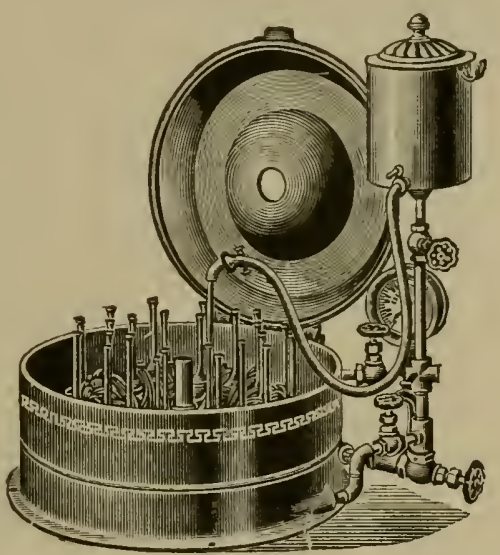

Frg. 22. Type of Babcock steam turbine tester's. of wire, so that it will not be broken by the centrifugal force and cause accidents. The swinging pockets which hold the test bottles in most machines should be so made that the bottles will not strike the center of the revolving frame when in a horizontal position. Tests have often been lost by the end of the neck catching at the center, the bottles thus failing to take an upright position when the whirling stops.

7r. The exhaust steam pipe of turbine testers should not have too many turns or be much reduced in size from that of the opening in the tester. A free escape of the exhaust steam is necessary to prevent the steam from collecting in the test bottle chamber and overheating the test bottles when whirled (41). 
The cover of the tester should have an opening provided with a sliding damper or some arrangement by which it can be closed when desired. If whole milk or cream is being tested, this hole should be open so that a draft of air may enter the chamber during the whirling, and force the steam into the exhaust pipe. If skim milk is being tested, the opening in the cover should be closed. This shuts off the draft of air, and the exhaust steam heats the test bottles during whirling to $200^{\circ} \mathrm{F}$. in some cases. This high temperature aids in separating the fat in skim milk and gives fairly

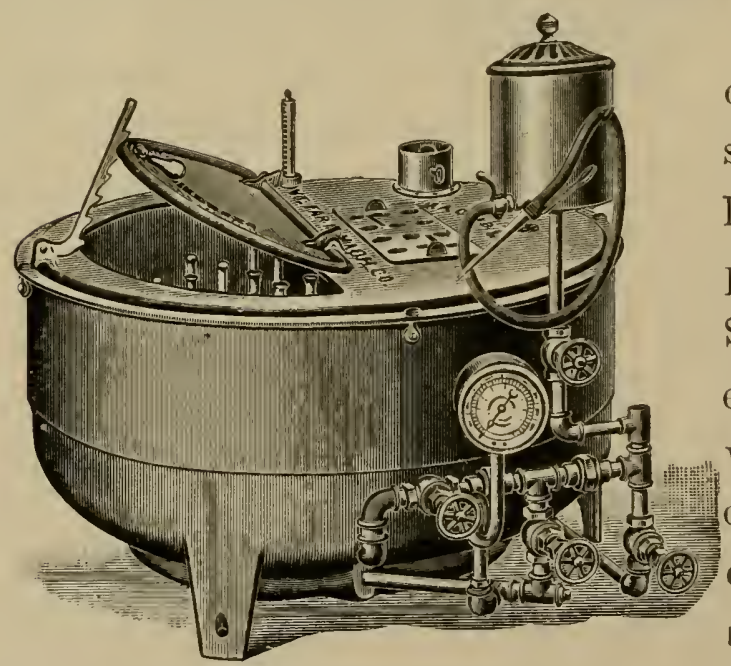

correct tests of samples containing less than one-tenth per cent. fat (98). Some turbine testers are provided with holes in the covers and dampers and a thermoneter is placed in F'IG. 24. Type of Babcock turbine testers (for testing cream in 9-in, cream bottles).

the cover. 
Babcock testers run by electricity have lately been put on the market by a couple of manufacturers (fig. 25). Where no steam, but electrical current is available, these machines m a y be installed to great advantage, as they are convenient to use and may be depended on to run at the required speed. Some provision for getting hot

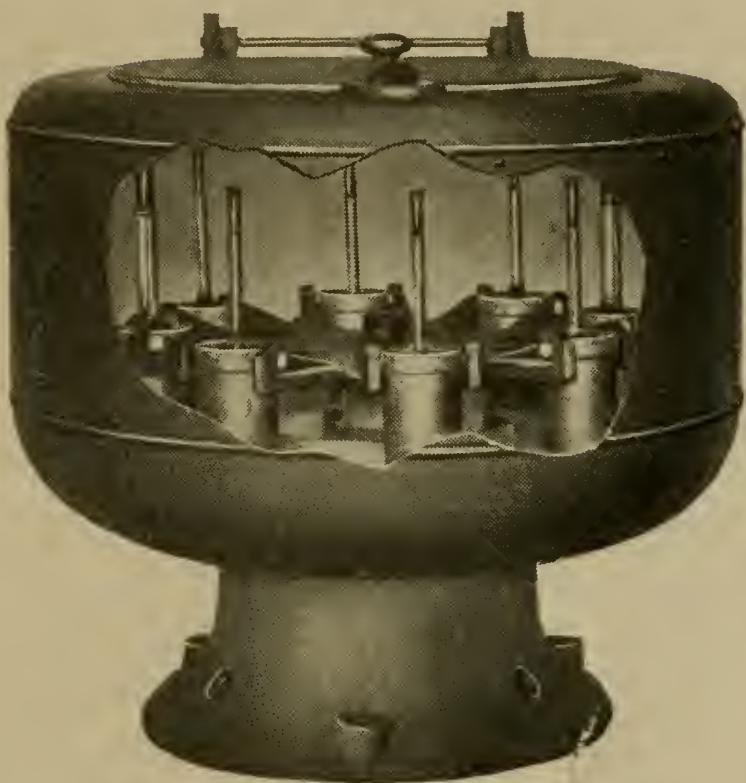
water must be at hand in using electrical Babcock testers. ${ }^{1}$

\section{3.- Sulfuric Acid.}

72. The sulfuric acid to be used in the Babcock test should liave a specific gravity of 1.82-1.83. ${ }^{2}$ Commercial sulfuric acid (sometimes called oil of vitriol) is always used; it can be bought for about 2 cents a pound in carboy lots and 25 cents or less a quart at retail. One quart of acid is sufficient for fifty tests. The acid should be kept in glass bottles or jugs, preferably glass or rubber stoppered ones, since a cork stop-

1 The method of installation of a 40 -bottle electrical Babcock tester is described in detail in Rept. Dept. of Health, City of Chicago, 1906, p. 1S.

${ }^{2}$ A specific gravity of 1.82 means that a given volume of the acid weighs 1.82 times as much as the same rolume of water at the same temperature (see also under Lactometer, 109). 
per is soon dissolved by the acid and rendered useless. If the bottle is left uncorked, the acid will absorb moisture from the air and after a time will become too weak for use in this test.

Lead is the only common metal which is not dissolved by strong sulfuric acid; where considerable milk testing is done, it is therefore desirable to provide a table covered with sheet lead on which the acid may be handled. The acid dissolves iron, tin, wood and cloth, and burns the skin. If acid is accidently spilled, plenty of water should be used at once to wash it off. Ashes, potash, soda, and ammonia neutralize the action of the acid, and a weak solution of any one of these alkalies should be used after the acid has been washed off with water. The red color caused by the action of the acid on clothing can be removed by wetting the spot with weak ammonia water; the ammonia must, however, be applied while the stain is fresh, and is in its turn washed off with water.

73. Testing the strength of acid. The strength of the acid can be easily tested by the use of a balance like that shown in fig. 34 (91). A dry test bottle is weighed, and then filled with acid exactly to the zero mark, or to any other particular line of the scale. It is then again weighed accurately; the difference between the two weights will give the weight of the acid in the bottle. The bottle is then emptied and thoroughly rinsed with water (until the water has no longer an acid taste); it is then filled with water to the same line as before and weighed; the difference between this weight and that of the empty bottle gives the weight of the 
same volume of water as that of the acid weighed. The specific gravity of the acid is obtained by dividing the weight of the acid by the weight of the water. If the quotient comes between 1.82 and 1.83 the acid is of correct strength. The outside of the test bottle should always be wiped dry before the liquids are weighed. Unless great care is taken in measuring the acid and the water, and in weighing both these and the test bottle, the results obtained will not be trustworthy.

74. Acid that is a little too strong can be used by taking less than the required amount for each test, e. g., about $15 \mathrm{cc}$. Operators are warned against reducing the strength of the acid by adding water to it, as accidents may easily occur when this is done. A too strong acid can, if desired, be weakened by simply leaving the bottle uncorked for a time, or by pouring the acid into a bottle containing a small quantity of water. In the latter case the first portions of acid should be added carefully, a little at a time, shaking the bottle after each addition, so as not to cause it to break on account of the heat evolved in mixing the acid and the water. Never dilute sulfuric acid by pouring water into it.

A helpful suggestion for using acid that is too strong or would give a charred fat on account of high temperature of acid or milk, or both, has been made by $\mathrm{M}$. L. Holm, Assistant Chemist Chicago Dept. of Health, viz., to add 2 cc. of 80 per cent. glycerin ( 80 parts of commercial glycerin and 20 parts of water, by volume) to the milk sample, prior to adding the acid. ${ }^{1}$ The gly-

${ }^{1}$ American Food Journal, 1907, No. 7, p. 28 ; Hoard's Dairyman, Nov. 8,1907 . 
cerin protects the milk to some extent from the acid before the two are mixed, and a clear fat may thus often be secured under otherwise unfavorable conditions. The results appear not to be influenced by the addition of the glycerin.

75. If the acid is a little too weak, correct results may be obtained by using more than the specified quantity, say 20 cc. If a good test is not obtained with this quantity of acid, a new lot must be secured, as its specific gravity in such a case is below 1.82. The observing operator will soon be able to judge of the strength of the acid by its action on milk in mixing the two liquids in the Babcock test bottles; it is indeed remarkable what slight differences in the specific gravity of the acid will make themselves apparent in working the test, as regards the rapidity with which both the curdled milk is dissolved and the mixture of acid and milk turns black.

76. Strength of sulfuric acid. The relation between the strength of sulfuric acid and its specific gravity will be seen from the following table:

Specific Gravity of Sulfuric Acid of Different Strength.

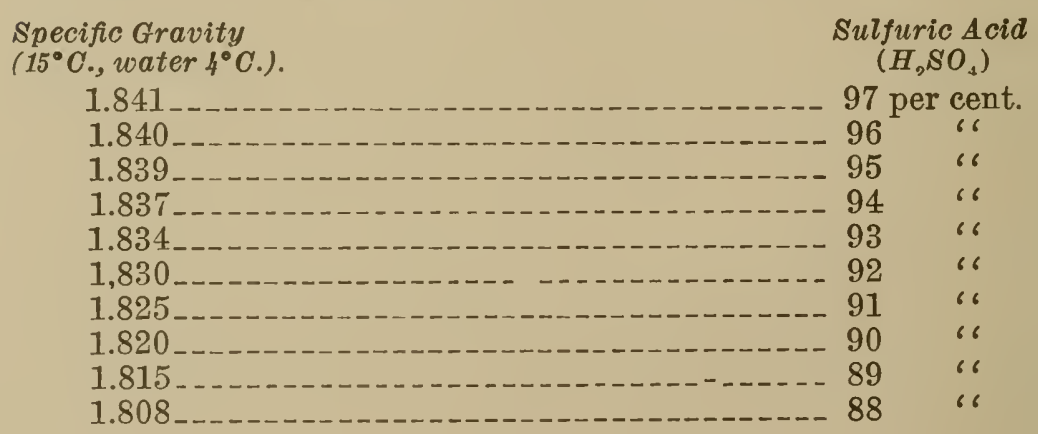


It will be noticed that the sulfuric acid to be used in the Babcock test should contain 90 to 92 per cent. of acid $\left(\mathrm{H}_{2} \mathrm{SO}_{4}\right)$; slightly weaker or stronger acid than this may, as previously stated, be used by varying the quantity of acid taken for each test according to the strength of the acid, but successful tests cannot, as a rule, be made with acid weaker than 89 per cent. or stronger than 95 per cent.

77. The Swedish acid tester is a small hydrometer, intended to show whether the acid used in the Babcock test is of the correct strength. An examination of these testers will show that they are practically useless for the purpose intended, from the fact that they are not sufficiently sensitive; while the testers examined were found to sink to the line marked Correct on the scale, when lowered into sulfuric acid of a specific gravity of 1.83, they would sink to a point much nearer the same mark, than to the lines marked Too strong or Too weak, respectively, when lowered into either too strong or too weak acid.

78. The color of the fat column an index to the strength of the acid used. The strength of the acid is indicated to a certain extent by the color of the fat which separates in the neck of the test bottle when milk is tested. If the directions given for making the tests are carefully followed, the fat separated out will be of a golden yellow color. If the fat is light colored or whitish, it generally indicates that the acid is too weak, and a dark colored fat, with a layer of black material beneath it, shows that the acid is too strong, provided the temperature of both milk and acid is about $70^{\circ}$. [For influence of temperature, see next paragraph.] The acid used in the test is not of sufficient strength to appreciably attack the fat at ordinary temperatures of testing, but a variation in the strength of the acid 
or in the temperature of the milk influences the intensity of the action of the acid on the fat, as shown in the color of the fat obtained.

The following experiment shows the relation between the strength of the acid, the temperature of the milk, and the color of the fat:

First:-From a sample of milk measure the usual quantity for testing into each of three bottles, A, B and C. Place A in iced water, and $\mathrm{C}$ in warm water, leaving bottle $\mathrm{B}$ at ordi. nary temperature. After the bottles have been left for ten minutes under these conditions, add the normal quantity of acid to each and proceed with the test in the ordinary manner.

Second:-Measure some of the same milk into three other bottles, $\mathrm{D}, \mathrm{E}$ and $\mathrm{F}$. Into test bottle $\mathrm{D}$ pour the usual amount of rather weak acid; add the same amount of acid of normal strength (1.82-1.83) to bottle $\mathrm{E}$, and add $17.5 \mathrm{cc}$. of a still stronger acid (concentrated sulfuric acid, sp. gr. 1.84), in test bottle $\mathrm{F}$; complete the tests in the usual way.

On the rompletion of the preceding six tests the operator will notice that the fat in the necks of test bottles A (cold milk) and $\mathrm{D}$ (weak acid) is much lighter colored than that in $\mathrm{C}$ (warm mill) and F (strong acid), and that the color of the fat in B (normal temperature) and $\mathrm{E}$ (normal acid) is somewhere between that of these two series.

79. Influence of temperature on the separation of fat. The intensity of the action of the sulfuric acid on the milk is influenced by the temperature of either liquid; the higher the temperature, the more intense will be the action of the acid on the solids of the milk. It may be noticed that acid from the same carboy will act differently on milk in summer than in winter time, if the acid and the milk are not brought to a temperature of about $70^{\circ}$ before testing during hoth seasons. The temperature of the liquids may be as low as $40^{\circ} \mathrm{F}$. in winter and as high as $80^{\circ} \mathrm{F}$. in summer. This dif- 
ference of forty degrees will often have considerable influence on the clearness of the fat separated, showing white curdy substances and a light colored fat in winter, or black flocculent specks, with a dark colored column of fat in summer. Both these defects can be avoided, when the acid is of the proper strength, by bringing the temperature of the milk and the acid to about $70^{\circ} \mathrm{F}$. before the milk is tested.

The operator should be particularly cautious against over-heating either milk or acid, since the heat intensifies the action of the acid and this may become so violent as to force the hot liquid out of the neck of the test bottle when the acid is added to the milk, thus spoiling the test and possibly causing an accident.

\section{4.-Water to be Used in the Babcock Test.}

80. Rain water, condensed steam, or soft water should be used for the purpose of bringing the fat into the neck of the test bottles. The surface of the fat column will then usually be clear and distinct. The foam or bubbles that sometimes obscure the upper line (meniscus) of the fat, making indistinct the point from which to measure it, is generally caused by the action of the acid on the carbonates in hard water. The carbonic acid gas liberated from such water by the sulfuric acid is more or less held by the viscid fat and produces a layer of foam on its surface. If clean soft water cannot be obtained for this purpose, hard water may be used, by adding a few drops of sulfuric acid to the water before it is heated, thus causing the carbonic acid to be ex- 
pelled. By simply boiling, many hard waters will be rendered soft and adapted to use in the Babcock test, as most of the carbonates which cause this foaming are thereby precipitated.

If the test has been completed, and a layer of foam appears over the fat, it may be destroyed by adding a drop or two of alcohol. If this is done, the fat column should be read at once after the alcohol is added, as the latter will soon unite with the fat and increase its volume. See also 96 on the use of glymol in cream testing.

81. Reservoir for water. When only a few tests are made at one time, hot water can be added with the 17.6 ce. pipette. If many tests are made, the water may be conveniently and quickly filled into the test bottles by drawing it from a small copper reservoir or tin pail suspended over the testing machine. ${ }^{1}$ The flow of water through a rubber tube connected with the reservoir, is regulated by means of a pinch cock. The water must be hot when added to the test bottles so as to keep the fat in a melted condition until the readings are taken. Most turbine testers are now made with a very convenient water reservoir attached to the tester (figs. 22-24).

The use of zinc or steel oilers, or perfection oil cans has been suggested as a convenient method of adding hot water to the test bottles, but most operators prefer to add water to the bottles by means of a piece of rubber tubing connected with a reservoir, as shown in the illustrations just referred to.

1 Ordinary tinware will soon rust with water standing in it, and copper reservoirs are therefore more economical. 


\section{5.-Modifications of the Babcock 'lest.}

82. The Russian milk test. The same chemical and mechanical principles applied in the regular Babcock test, are used in the Russian milk test, except that in this case the machine in which the bottles are whirled, and the bottles themselves, are so constructed that the latter can be filled with hot water while the machine is running ${ }^{1}$, thus saving time and the trouble ineident to the stopping of the tester and filling the bottles by means of a pipette. The milk-measuring pipette (fig. 28) and

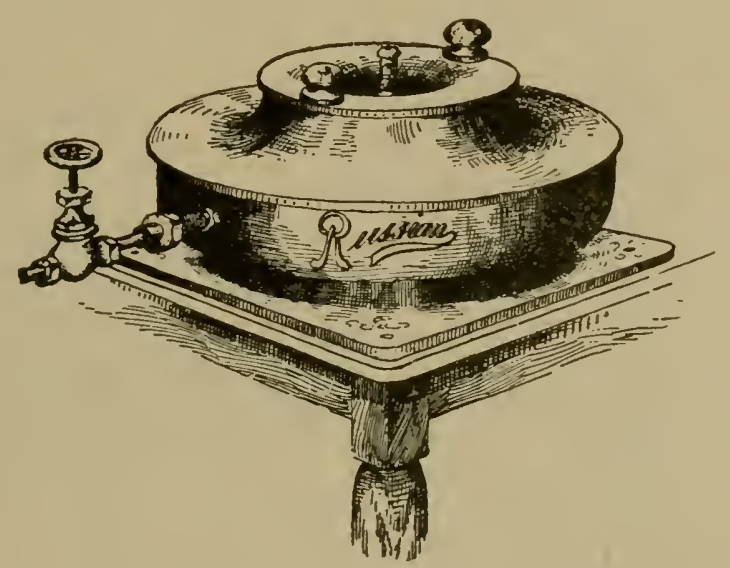

FIG. 26. The Russian test.

the acid measure used in the Russian test are one-half of the ordinary size, and the test bottles are made in two pieces with a detachable narrow graduated stem (see fig. 27). The machine is substantially made of cast iron; it is provided with a speed indicator which shows at any time the number of revolutions at which the bottles are being turned. The accompanying illustrations show the apparatus used in this test. When the directions for operating the test are followed closely, the results obtained are accurate and very satisfactory.

1 A similar arrangement for the regular Babcock test has been suggested by Mitchell and Walker of Kingston (Ont.) Dairy School (see Ont. Dept. of Agriculture, bull. 170). 
83. Bartlett's modification. Bartlett ${ }^{1}$ proposed a modification of the method of procedure in the Babcock test, which aims to simplify the manipulations. 20 ce. of acid are added, instead of $17.5 \mathrm{cc}$, and the bottles filled with the milk-acid mixture are left standing for not less than five minutes and then filled with hot water to within the scale; the bottles are then whirled for five minutes at the regular rate (52).

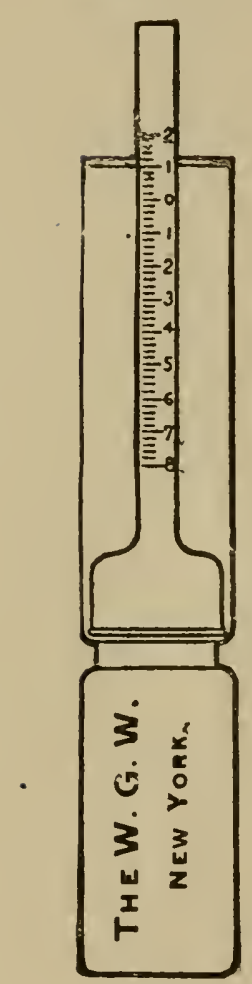

FIG. 27.

Test bottle used in the Russian test.

83a. Siegfeld's modification. The German dairy chemist Siegfeld in 1899 proposed a modification of the Babcock test, ${ }^{2}$ using 2 cc. of amyl alcohol with the sulfuric acid, and filling up with dilute sulfuric acid (1:1, sp. gr., 1.5) in one filling, in place of water after the whirling. A clear separation of the fat is facilitated by both these changes, but when properly conducted there is no difficulty whatever in obtaining a clear fat columu in the Babcock test as described in this book, and the modification has not therefore been generally introduced in American factories. It has, however, been adopted in many German creameries where the Babcock test is used.

\section{Bausch and Lomb centrifuge.} Fig. 29 shows a form of hand centrifuge which may be used to advantage by physicians or in pathological laboratories for the determination of fat in

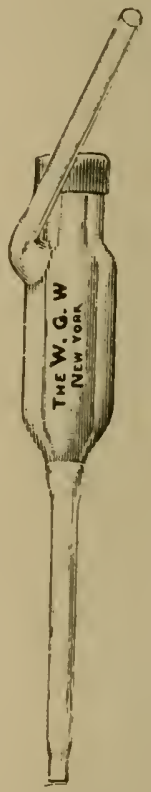

FIG. 28. Pipette used in the Rus-. sian test. milk. The centrifuge is especially designed for examination of urine, sputum, blood, etc., but has been adapted to milk analysis by the Leffmann \& Beam test, a special form of bottle (fig. 30) having been constructer for this purpose. The machine gives satisfactory results by the Babcock test as well, provided the acid used is 1.83-1.84, or if the bottles containing the acid-milk mixture be placed in hot water for five or ten minutes prior to the whirling.

${ }^{1}$ Maine experiment station, bull. 31 (s. s.)

2 Jolkerei Ztg., 1899 , p. 51. 
As the bottles are calibrated for only $5 \mathrm{cc}$. of milk and the neck of the bottles, with scale, is correspondingly fine, testing milk with this machine requires some nicety of manipulation not called for in case of regular Babcock testers constructed for the use of factory operators and dairymen.
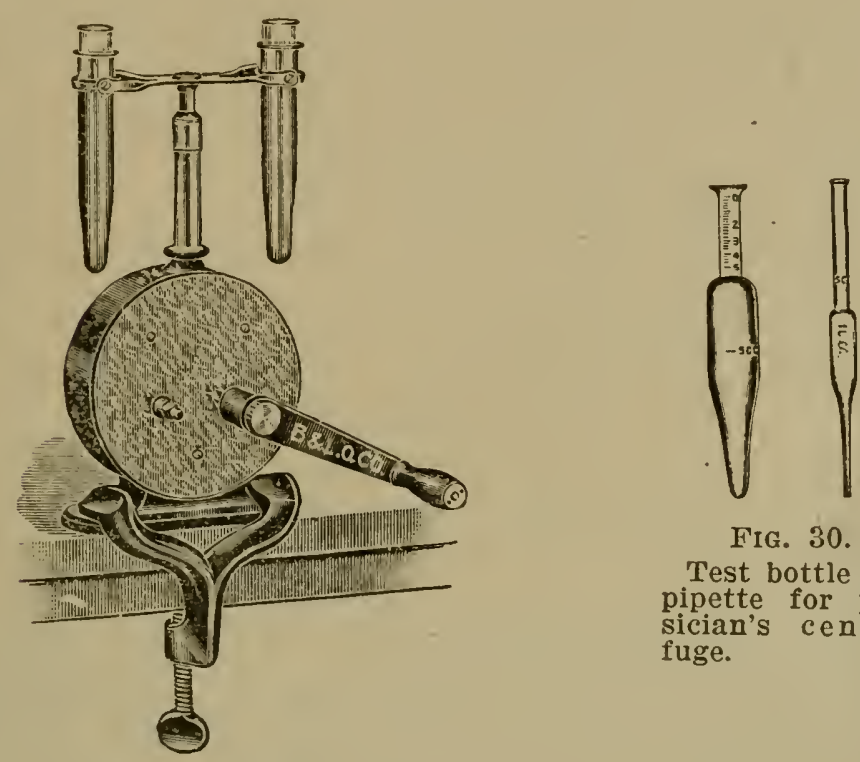

Fig. 30.

Test bottle and pipette for physician's centrifuge.

FIG. 29. Physician's centrifuge that may be used for milk testing.

840. Whitman milk bottle. Dr. Ross C. Whitman has devised a milk testing bottle for the special use of physicians in testing human milk and small amounts of cow's milk by the Babcock test. ${ }^{1}$. The bottle, which ean be placed in an ordinary urine centrifuge, consists of two parts, a small tube for holding the milk, acid and water, and a detachable graduated fine tube, into which the fat column is brought after the final filling and whirling. 5cc. of milk are used in this test.

\footnotetext{
${ }^{1}$ Jour. Amer. Med. Asso., 47 (1906), p. ㅁ04.
} 


\section{Questions.}

1. Give a brief description of the Babcock test.

2. State precautions to be observed in each of the following operations: (a) Measuring the milk, (b) adding the acid, (c) whirling the bottles, (d) adding the water, (e) measuring the fat. If the fat separates clear, but the results are evidently too low, what is the probable cause, and how can the correct test be established?

3. To what extent does the temperature of the fat, when read, influence the result?

4. Explain the graduations of the milk test bottle.

5. What is the capacity of the neck of a milk test bottle between the 0 and 10 marks, expressed in cc., and in grams?

6. If the graduations of a test bottle measure 2.3 cc. from 0 to $10 \%$, what would be the correct test of a sample which reads $3.4 \%$ fat in this bottle?

7. Describe three different methods of calibrating milk test bottles.

8. Describe the proper construction of the milk-measuring pipette; what weight of milk does it deliver?

9. What is a Swedish acid-bottle?

10. What speed is required for testers having a diameter of 8,15 , and 20 inches:

11. Write an order for one gallon of sulfuric acid to be used in testing.

12. How can the strength of the acid be tested at the farm or in a factory?

13. State precautions to be taken in using an acid that is (a) too strong, (b) too weak.

14. What does the color of the fat indicate in regard to the strength of the ac:d or the temperature of either acid or milk?

15. What is the cause of foam above the fat column, and how may it be prevented?

16. What causes white curd or black specks in the fat?

17. Describe a few modifications of the Babcock test.

18. In which two points does the Russian milk test mainly differ from the Babcock test? 


\section{CHAPTER IV.}

\section{CREAM TESTING.}

85. Cream may be tested by the Babcock test in the same manner as milk, and the results obtained are accurate when the necessary care has been taken in sampling the cream and measuring the fat. 'The composition of cream varies greatly according to the process of creaming, the temperature of the milk during the creaming, the quality and the composition of the milk, etc. The cream met with in separator creameries will contain from 25 to 40 per cent. of fat, or on the average about 35 per cent. Cream from hand separators may be as rich as this, but it often contains only 20 per cent.
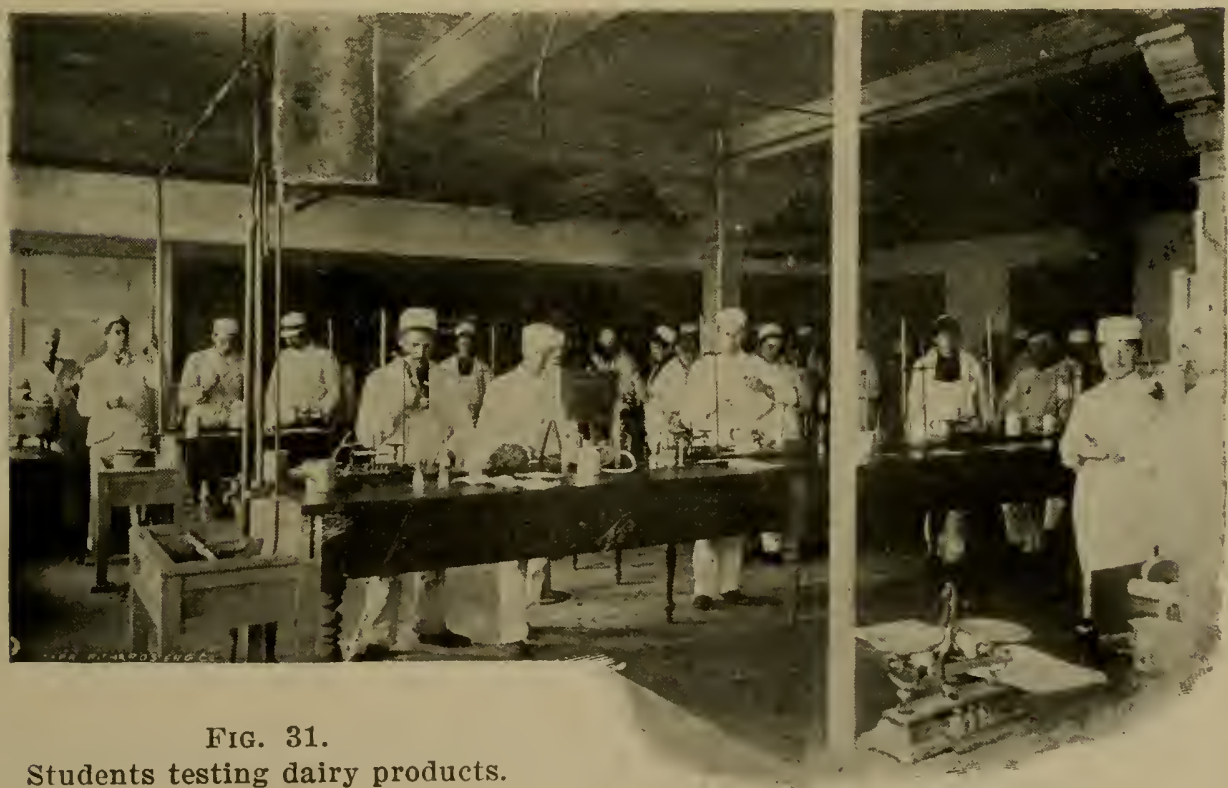

Students testing dairy products. 
of fat as delivered to creameries. An average grade of market cream as retailed contains about 25 per cent. of fat. If 18 grams of 25 per cent. cream is measured into an ordinary Babcock test bottle, there will be $18 \times .25=4.5$ grams of pure butter fat in the bottle, or, (since the specific gravity of butter fat is about .9) $\frac{45}{.9}=5$ ce. It is shown that the space from 0 to 10 in the neck of these bottles holds exactly 2 cc. (44). The neck of the milk test bottle is not large enough to show the per cent. of fat in a sample of cream if 18 grams are taken for testing, and it is therefore necessary to adopt special measures when cream is to be tested.

86. Errors of measuring cream. Several factors tend to render inaccurate the measuring of cream for the Babcock test, and correct results can therefore only be obtained by weighing the cream. If a 17.6 cc. pipette is used in testing the cream, it will not deliver 18 grams of cream, as.it will of milk, for the following reasons :

1. The specific gravity of cream is lower than that of milk; if a certain quantity of milk weighs 1030 lbs., the same quantity of cream will weigh from 1020 lbs. to $1000 \mathrm{lbs}$. or less, the weight being determined by the richness of the cream; the more fat the cream contains, the less a certain quantity of it, e. g., a gallon, will weigh. ${ }^{1}$

2. Cream is thicker (more viscous) than milk at the same temperature, and more of it will adhere to the sides of the measuring pipette than in the case of milk.

${ }^{1}$ For specific gravity of cream of different richness, see table on p. 7 . 
This is of special importance in testing very rich or sour cream.

3. In case of separator cream, more or less air will become incorporated with the cream during the process of separation. In the ripening of cream, the fermentation gases developed are held in the cream in the same way as bread dough holds the gases generated by yeast. In either case the weight of a certain measure of cream is diminished.

87. As an illustration of the effect of the preceding factors on the amount of cream measured out by a Babcock 17.6 cc. pipette, the following weighings of separator cream are given (column b.) The cream was in all cases fresh from the separator; it was weighed as delivered by the pipette into a cream test bottle (89), and the test proceeded with at once; the specific gravity of the crcam was determined by means of a picnometer (248). The data given are in all cases averages of several determinations; the samples of cream have been grouped according to their average fat contents. ${ }^{1}$

$\begin{array}{cc}\begin{array}{c}\text { Per cent. } \\ \text { of fat. }\end{array} & \\ \text { in cream. } & \text { Specific gravity }\left(1.75^{\circ} \mathrm{C} .\right) \\ 10 & \text { (a) } \\ 15 & 1.023 \\ 20 & 1.012 \\ 25 & 1.008 \\ 30 & 1.002 \\ 35 & .996 \\ 40 & .980 \\ 45 & .966 \\ 50 & .950 \\ & .947\end{array}$

Weight of cream deliv. ered, grams.

17.9

17.7

17.3

17.2

17.0

16.4

16.3

16.2

15.8

${ }^{1}$ For influence of condition of cream on the amount measured out with a $\mathbf{1 7 . 6}$ cc. pipette, see also Bartlett, Maine exp. sta., bull. 31 (S. S.) ; Jones, Vt. exp. sta., report 16, 101-6, and Dean, Guelph (Ont.) agr. college, report 1906, p. 125. 
The figures in the table show plainly the variations in the specific gravity of cream of different richness and the error of making tests of cream by measuring it with a 17.6 cc. pipette, especially if the pipette is not rinsed and the washings added to the test bottle. If the cream to be sampled is fresh separator cream testing over 30 per cent., less than 17.0 grams of cream will be delivered into the test bottle, and the results of the reading will be at least one-eighteenth too low (since the bottles are graduated for 18 grams), or about 1.6 per cent. too low in the case of a 30 per cent. cream. If the cream is sour, the error will of course be still greater.

It should be remembered that the specific gravities of the cream given in the table refer to fresh separator cream only. Considerable air is incorporated during the separation, and cream of this kind is therefore lighter than gravity cream of corresponding fat contents.

88. Weighing cream for testing. For the reasons stated in the preceding, accurate tests of cream can only be made by weighing the cream into the Babcock test bottles. ${ }^{1}$

The simplest method is to weigh 9 or 18 grams of the samples on a small cream-weighing scale (see p. 81 ) into one of the special forms of cream-test bottles.

Cream-test bottles. Special forms of bottles have been devised for testing samples of cream by the Babcock test by Winton, Bartlett, and by various manufacturers.

1 This is recognized by a law passed by the Wisconsin legislature in 1903, which requires cream to be weighed for testing where it is sold on the basis of its fat content. (Chapter 43. Iaws of 1903 , An act to prescribe the standard measures for the use of the Babcock test in determining the per cent. of butter fat in milk or cream.) 
89. The Winton cream bottle. The cream-test bottle devised by Winton, ${ }^{1}$ (fig. 32), has a neck of the usual length, and of sufficient width to measure 30 or 50 per cent. of fat. The scale of the neck is divided into parts representing one-half of one per cent. each, but readings of a quarter of a per cent. can easily be estimated. Such readings of cream tests are sufficiently exact for most commercial purposes. This form of cream bottle will be found very convenient in making tests of composite samples of cream.

Cream test bottles of a small bore are greatly to be preferred to those with wide necks (fig. 33), since they permit of accu-

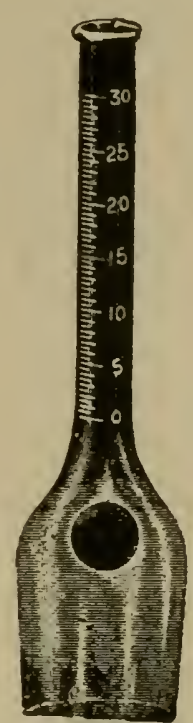

FIG. 32. The $30 \%$ cream test bottle. rate readings to a quarter of a per cent.

Other forms of cream-test bottles which allow the testing of 50 or 55 per cent. cream have been placed on the market during late years by some manufacturers. These bottles (so-called 9-inch bottles) have long necks FIG. 32a. The $50 \%$ )-inch cream test bottle. and require especially constructed, large and deep testers (see fig. 25). These machines and accompanying bottles have of late been adopted for cream testing in many localities where farm separator cream is delivered to the creameries.

${ }^{1}$ Connecticut experiment station (New Haven), bull. 117 ; report 1894, p. 224. 
90. The bulb-necked cream test bottles allow the testing of cream containing 23 or 25 per cent. of fat, when the usual quantity of cream (18 grams) is taken. The neck is graduated from 0 to 23 per cent., and in some cases to 25 per cent., the graduation extending both below and above the bulb. This is sometimes an inconvenience, as the water must be added

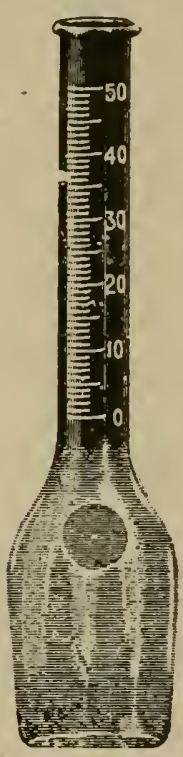

FIG. 33 .

The $50 \%$ cream test bottle. carefully so that the lower end of the column of fat will always come below the bulb, in the graduated part of the neck, and not in the bulb itself. Beginners are especially apt to lose tests when this bottle is first used, for the reason given. It is recommended to fill these bottles with the first portion of hot water to just above the bulb, so that one can see how much water to add the second time in order to bring the fat within the scale.

Each division of the scale on these cream bottles represents two-tenths of one per cent. of fat, as in case of the milk test bottles. This form of bottle is no longer used to any extent, as it has been largely re. placed by the different forms of the Winton cream-bottle.

\section{Scales for weighing the} cream. When a small, delicate balance is used, cream can be weighed rapidly into the bottles. Either. of the scales shown in the accompanying illustrations, (figs. 34-35), will be found sufficiently accurate for this purpose; a small scale of this kind is also convenient and helpful in testing cheese, butter and condensed milk, in determining the strength of sulfuric acid, and in testing the accuracy of test bottles and pipettes.

The cream scale shown in fig. 35 permits

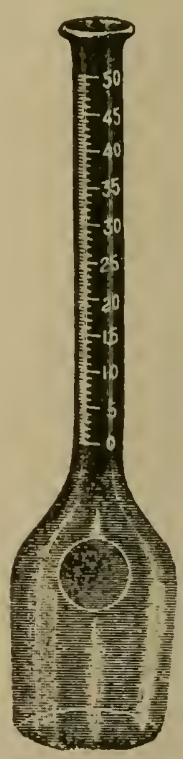

FIG. $33 a$. The $50 \% 9$ gram cream test bottle. the weighing of six samples of cream on each pan with 
only one taring of the bottles, which greatly facilitates the work of testing the cream.

In testing cream by weight, the test bottle is first weighed empty, and again when 9 or 18 grams of cream have been placed in it; the difference between the two figures gives the

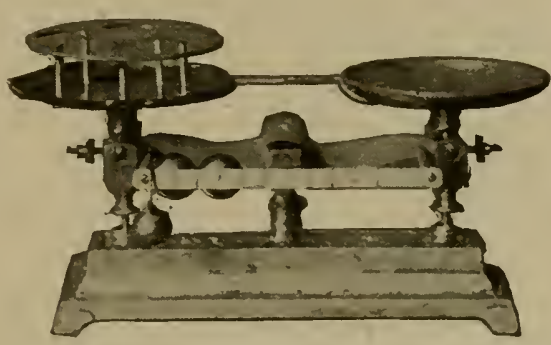

Frg. 34. Scales used for weighing cream in the Babcock test. weight of cream taken for the test. If the cream contains less than 30 per cent. of fat, the regular milk test

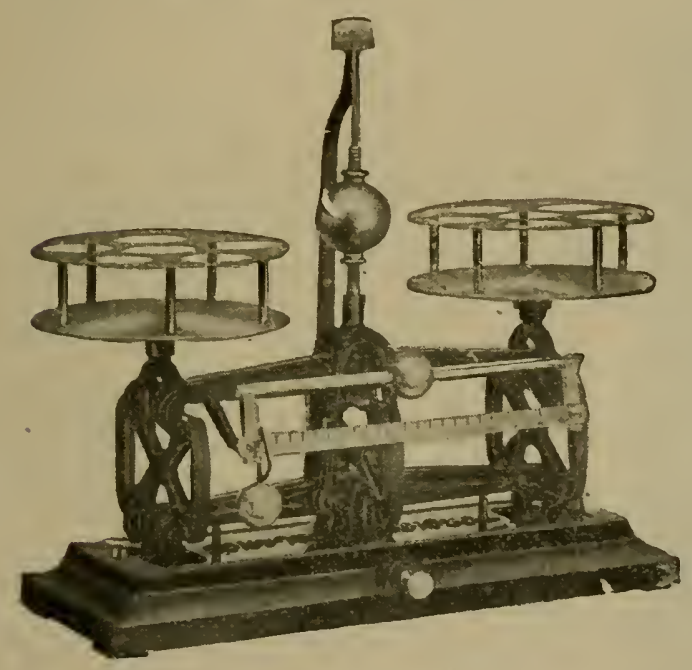

FIG. 35. Torsion balance used for weighing cream in the Babcock test.

bottle can also be used for testing the cream, if not much more than 5 grams are weighed out; if more cream is taken, or if this is richer than 30 per cent., it is advisable to use cream bottles.

The operatol should be careful in weighing the cream not to spill it on the outside of the test bottle. If less than 18 grams of cream has been weighed into the bottle sufficient water is added to the balance to make the total volume about $18 \mathrm{cc}$. The usual quantity of acid ( 17.5 cc.) is then added, and the test completed in the 
ordinary manner. The reading of the amount of fat in the neck of the test bottle. will not show the correct per cent. of fat in the cream unless exactly 18 grams are weighed out. If less than this weight was taken the per cent. of fat in the cream tested is obtained by multiplying the reading by 18 , and dividing the product by the weight of cream taken.

EXAMPLE: Weight of cream tested, 5.2 grams; reading of column of fat $\left.\left.{ }^{1}\right) 9.8,{ }^{2}\right) 9.7$, average 9.75 ; per cent. of fat in the cream $\frac{9.75 \times 18}{5.2}=33.75$.

It is very convenient to weigh out 18 grams of cream (or 9 grams) so that the readings may be taken di-

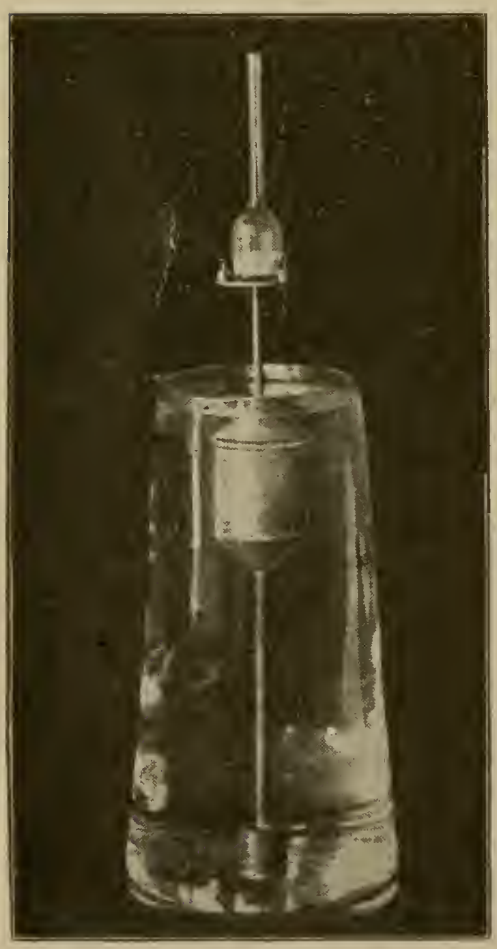

FIG. 35a. The Wisconsin hydrostatic cream balance. rectly from the neck of the bottle. The smaller the quantity of cream taken for a sample, the greater is the error introduced by inaccurate weighings or readings. The result is rendered more accurate if two or three tests of a sample are made, and the readings averaged.

9ra. The hydrostatic balance is a convenient device for weighing cream and other dairy products to be tested by the Babcock test (see fig. 35a). ${ }^{1}$ This balance is built on the same principle as a lactometer : it is pro-

\footnotetext{
1 Wisconsin exp. station, bull. 195.
} 
vided with a pan on the top of the stem, on which the test bottles and the weights are placed. When put into water the instrument is balanced to a certain point with empty test bottles and weights on the pan; the weights are then removed and sufficient cream added to the test bottle by means of a pipette to sink it to the same point.

The special advantages of the balance are that there are no bearings to rust and become dull; it is durable, inexpensive and sensitive, and with careful handling will remain sensitive indefinitely. The balance can be made large enough to weigh a number of bottles at a time, as is the case with some of the cream scales on the market.

92. Measuring cream for testing. Where a special cream scale or a small balance is not available, fairly satisfactory results may be obtained with cream of low or average quality by measuring out the sample with a 17.6 pipette and correcting the results as indicated below. One of the cream test bottles or a common milk test bottle may be used for this purpose. The table cn p. 77 shows that a 17.6 cc. pipette, in the case of cream fresh from the separator, containing less than 25 per cent. of fat, will deliver only 17.2 grams of cream, that is, the results will be $\frac{.8 \times 25}{18}=1.1$ per cent. too low. In the same way in case of 40 per cent. cream, only 16.3 grams of cream would be delivered, and the results therefore 3.8 per cent. too low. When the cream has been ripened or is thick, less cream would be delivered than the amounts given, and the error introduced by measuring out the samples correspondingly increased. 
A table of correction for testing such cream by measuring the samples has been prepared by Prof. Eckles, formerly of Iowa experiment station. ${ }^{1}$

Approximately correct results may be obtained in testing thin cream by using an 18 cc. measuring pipette; to avoid the expense and trouble of using two different pipettes, one for milk and one for cream, a pipette with two marks on the stem, at $17.6 \mathrm{cc}$. and 18 cc., has been placed on the market, the former mark being used when milk is tested, and the latter for cream. It should be borne in mind, however, that such pipettes can only be used in the case of sweet cream of average richness, and will then give only approximately correct results.

93. Use of milk test bottles. Cream may be tested by emptying a $17.6 \mathrm{cc}$. pipetteful into two or more milk test bottles, dividing the sample about equally between the bottles and filling the pipette with water once or twice, which is then in turn divided about equally between the test bottles; the per cent. of fat in the cream is found by adding the readings obtained in each of the bottles. The cream and the water must be mixed before the acid is added.

1 Press bull. dated August, 1901. Some creameries heat the samples of cream in a water bath to about $140^{\circ} \mathrm{F}$. before the test samples are measured out by means of a $\mathbf{1 7 . 6} \mathrm{cc}$. pipette. This increases the fluidity of the cream and causes less to adhere to the pipette. The Vermont cxperiment station (report 16, pp. 191-6) found in examining this method that it did not yield satisfactory results in the case of cream of different richness and lecommends that cream be weighed when accurate tests are desired.

Professor Spillman (Bull. 32 of Washington experiment station) recommends the use of a $\mathbf{1 7 . 6} \mathrm{cc}$. pipette for testing cream, the results obtained being corrected by a certain per cent., as shown in a table given in the bulletin. The table is based on the figures given on p. 77 of this book, and is therefore only applicable to fresh separator cream. 
This method does away with the error incident to the adhesion of cream to the side of the pipette, but not with that due to the low specific gravity of the cream, and the results obtained will therefore be too low.

The dilution of the cream with water in the test bottles not only makes it possible to bring into the bottle all the cream measured out, but also insures a clear test. If ordinary cream is mixed with the usual quantity of sulfuric acid used in the Babcock test, a dark-colored fat will generally be obtained, while the cream diluted with an equal or twice its volume of water, when mixed with the ordinary amount of acid, will give a light yellow, clear column of fat, which will allow of a rery distinct and sharp reading.

The number of bottles to be used for testing a sample of cream by this method must be regulated by the richness of the cream. If the sample probably contains 20 per cent. or more, a pipetteful should be divided about equally between three milk test bottles, and twothirds of a pipetteful of water is added to each bottle. If the cream contains less than 20 per cent. of fat, it will only be necessary to use two milk test bottles, dividing the pipetteful between these, and adding one-half of a pipetteful of water to each bottle.

By using cream test bottles (89), more accurate tests may be obtained in case of cream containing as much as 25 per cent. of fat, by dividing one pipetteful between two bottles, rinsing half a pipette of water into each one, than by adding all the cream to one bottle without rinsing the pipette, for reasons apparent from what has been said in the preceding. 
94. Use of a 5 cc. pipette. When the cream is in good con dition for sampling, satisfactory results can also be obtained by the use of a 5 ac. pipette, provided great care is taken in mixing the cream before sampling; 5 cc. of cream are measured into a milk test bottle, and two pipettefuls of water are added. In this way all the cream in the pipette is easily rinsed into the test bottle. The readings multiplied by $\frac{18}{5}=3.6$ will give the per cent. of fat in the cream. If the specific gravity of the cream tested varies appreciably from 1, corrections should be made accordingly; e. g., if the specific gravity is 1.02 , the factor should read $\frac{18}{5 \times 1.02}=3.53$; if $.95, \frac{18}{5 \times .95}=3.79$, etc.

95. Proper readings of cream tests. The accompanying illustration (fig. 36), shows the proper method

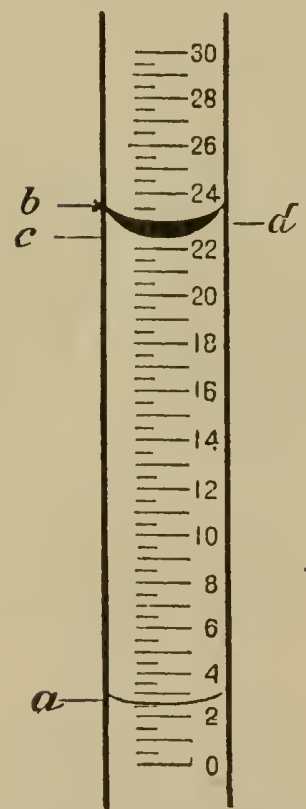

Frg. 36. Measuring the fat column in the neck of a cream bottle. Reading should be made from $a$ to $c$, not to $b$ or to $d$. of reading the fat column in cream tests; readings are taken from $a$ to $c$, not to $b$ or to $d$, when readings are made at $140^{\circ} \mathrm{F} \cdot{ }^{1}$

No special precautions other than those required in testing milk have been found necessary in testing cream, except that it is sometimes advisable not to whirl the test bottles in the centrifuge at once after mixing, but to let the cream-acid mixture stand for a while, until it turns dark colored. At first, the mixture of cream and acid is much lighter colored than that of milk and acid, owing to the smaller proportion of solids not fat contained in the cream.

The liquid beneath the fat in a completed test of cream is sometimes milky

1 The size of the meniscus is magnifled in this cut. A study of the mensicus formed in bottles with narrow or wide necks, and its bearing 
and the fat appears white and cloudy, making an exact reading difficult. Such defects can usually be overcome by placing the test bottles in hot water for about ten minutes previous to the whirling, or by allowing the fat to erystallize (which is done by cooling the bottles in cold water after the last whirling) and remelting it by placing the bottles in hot water.

'The error due to the expansion of the fat in case of excessively hot turbine testers having no openings in the cover as mentioned on p. 36 , is especially noticeable in cream testing, where it may amount to one per cent. or more. In order to obtain correct results with such testers, the hot cream test bottles must be placed in water at about $140^{\circ} \mathrm{F}$. for some minutes before the results are read off.

The subject of different methods of reading cream tests have been studied by Webster and Gray, ${ }^{1}$ who conclude that correct results are obtained by taking readings at $120^{\circ} \mathrm{F}$., from the bottom to the extreme top of the fat column, deducting four-fifths of the depth of the meniscus from this result and adding .2 per cent. to the figure thus obtained.

96. Eliminating the meniscus in cream tests. The uncertainty concerning the exact point at which the meniscus of the fat column should be read in cream tests has been removed by the use of certain liquids which do not mix with fat but when dropped on top of

on the results of cream tests is given in bulletin 58, Bur. An. Ind., U. S. Dept. of Agriculture, where a discussion of the influence of different temperatures on readings of cream tests will also be found (see 96).

${ }^{1}$ Bull. 58, Bur. An. Ind., U. S. Dept. of Agriculture. 
the fat column change the curved surface of the meniscus into a straight line. Amyl alcohol, fat-saturated alcohol and glymol have been suggested for this purpose.

Amyl alcohol colored red by fuchsin was suggested by Eckles. ${ }^{1}$ This may dissolve some of the fat and thus cause a slightly low reading. Fat-saturated alcohol largely overcomes this objection. It is made by adding about a teaspoonful of butter to six ounces of denatured or wood alcohol in a stoppered bottle. This is warmed slightly and shaken until the alcohol does not dissolve any more of the fat. A small amount of

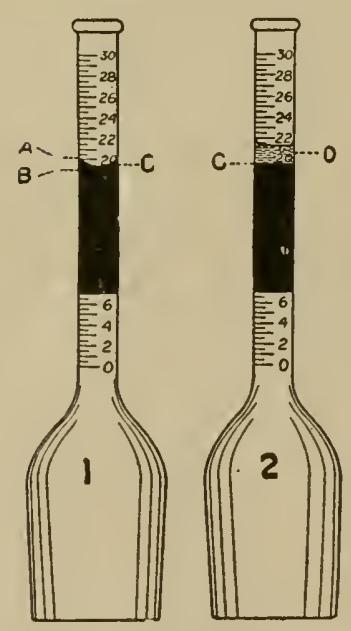

FIG. 36a. Showing the use of fat-saturated alcohol, glymol, etc., for eliminating the $\mathrm{m}$ e $\mathrm{n}$ is $\mathrm{cus}$ in cream tests. coloring matter may be added to this solution to further facilitate the reading. The use of glymol $^{3}$ or white mineral typewriter and sewing machine oil was suggested by Hunziker for reading cream tests, after an exhaustive study of the subject. Glymol may be colored with alkanet root which can be obtained from a druggist. One ounce of alkanet root will color one quart of glymol; this is done by placing a small cheese cloth bag filled with the alkanet root in the bottle of glymol for one or two days.

A convenient way of adding alcohol, glymol, etc., to the fat column after completing a test is to insert a

${ }^{1}$ N. Y. Produce Review, Aug. 8, 1908.

2 Wis. Expt. Sta. Bul. 195, p. 6.

3 Purdue, Ind., Expt. Sta. Bul. 145, vol. XV, p. 593. 
glass tube through a cork or stopper of a bottle containing the liquid and by placing a finger on the top of the tube a small portion may be transferred from the bottle to the top of the fat column. By the use of either of the liquids mentioned the meniscus in cream tests disappears, giving a straight line at the top as well as the bottom of the fat column and thus making it possible to obtain exact readings of the per cent. of fat in any sample of cream.

\section{Questions.}

1. Give three rcdsons for weighing cream for testing.

2. How does the richness of the cream influence its weight

3. What is the weight of one gallon of cream testing 10,30 , or $50 \%$ fat?

4. Describe at least three forms of cream test bottles.

5. What is the use of a bulb in the cream bottle.

6. Between what points should the cream fat column be read?

7. If cream was erroneously weighed into a test bottle as 9.3 gr. instead of $10 \mathrm{gr}$., what error would this cause on a sample testing $33 \%$ fat

8. Mention a few important points in the construction of a cream test bottle.

9. If $12.5 \mathrm{gr}$. cream give a reading of 18.5 , what is the correct test of the sample?

10. If $7.2 \mathrm{gr}$. of cream give a reading of 6.4 , what is the correct test of the sample?

11. If the fat in a cream test is read as $28 \%$ at a temperature of $180^{\circ} \mathrm{F}$., what is the correct test?

12. If at the end of a full day's run $4,280 \mathrm{lbs}$. of milk had been received, testing 3.95 per cent., and 535 lbs. of cream testing 34.5 per cent. fat; how much fat (a) in the whole milk; (b) in the cream; (c) in the skim milk (d) what would be the test of the skim milk, (e) how many pounds of skim milk would there be; and (f) what would be the per cent. of cream from the milk, 


\section{CHAPTER V.}

\section{BABCOCK TEST FOR OTHER MILK PRODUCTS.}

97. Skim milk. Each division on the scale of the neck of the regular Babcock test bottle represents twotenths of one per cent. (44). When a sample of skim milk or butter milk containing less than this per cent. of fat is tested, the estimated amount is expressed by different operators as one-tenth, a trace, one-tenth trace, or one- to five-hundredths of one per cent. Gravimetric rhemical analyses of skim milk have, however, shown that samples which give only a few small drops of fat Hoating on the water in the neck of the test bottle, or adhering to the side of the neck, generally contain one-tenth of one per cent. of fat, and often more. Samples of skim milk containing much less than one-tenth of a per cent. of fat are very rare, and it is doubtful whether a sample of separator skim milk representing a run of, say 5000 lbs. of milk, will ever show less than five-hundredths of one per cent. of fat. Under ordinary factory conditions, few separators will deliver skim milk containing under one-tenth of one per cent. of fat, when the sample is taken from the whole day's run. This must be considered a satisfactory separation. ${ }^{1}$

1 For comparative analyses of separator skim milk by the gravimetric method and by the Babcock test, see Wis. exp. station bull. 52 and rep. XVII, p. 81 ; Conn. (Storrs) exp. station, bull. 40 : Utah exp. station, bull. 96. See also, Woll, Testing Skim Milk by Babcock 'Test, in Country Gentleman, April 26, 1902. The results obtained by the use of the Gottlieb method have shown that ether-extraction methods, as 
98. The reason why the Babcock test fails to show all the fat present in skim milk must be sought in one or two causes: a trace of fat may be dissolved by the sulfuric acid, or owing to the minuteness of the fat globules of such milk they are not brought together in the neck of the bottles at the speed used with the Babcock test. The latter cause is the more likely explanation. If a drop of the dark liquid obtained in a Babcock bottle from a test of whole milk be placed on a slide under the microscope, it will be seen that a fair number of very.minute fat globules are found in the liquid. These globules are not brought into the column of fat in the neck of the bottle by the centrifugal force exerted in the Babcock test, even if the bottles are whirled in a turbine tester in which they are heated to $200^{\circ} \mathrm{F}$. or higher (see 71) ; the loss of the fat contained in these fine globules is compensated for, in the testing of whole milk, by a liberal reading of the fat column, the reading being taken from the bottom of the fat to the top of the upper meniscus (see p. 35); in some separator skim milk, on the other hand, not enough fat remains to completely fill the neck, and the reading must therefore be increased by at least fivehundredths of one per cent.

It follows from what has been said that tests of skim milk showing no fat in the neck of the test bottles on completion of the test, generally indicate inefficient work of the centrifugal tester or of the operator, or of

well as the Babcock test, give too low results with dairy by-products low in fat, like skim milk, butter-milk, etc. The Gottlieb method for this reason has been adopted by European chemists as a standard for analysis of these products. (See 254). 
both. The test should be repeated in such cases, using more acid and whirling for full five minutes. Separator skim milk should be allowed to stand 10 to $15 \mathrm{~min}$ utes before the sample is taken so as to allow the air to escape.

In order to bring as much fat as possible into the neck of the bottles in testing skim milk, it is advisable to add somewhat more acid than when whole milk is tested, viz., about 20 cc., and to whirl the bottles at full speed for at least five minutes, keeping the tester as hot as possible the whole time. ${ }^{1}$ The readings must be taken as soon as the whirling is completed, since owing to the contraction of the liquid by cooling, the fat will otherwise adhere to the inside of the neck of the test bottle as a film of grease which cannot be measured by the scale.

99: The double-necked test bottle, (fig. 37), suggested by one of us, ${ }^{2}$ is made especially for measuring small quantities of fa.t and gives fairly satisfactory results in testing skim milk and butter milk. Each division of the scale in these bottles represents five-hundredths of one per represents cent., and the marks are so far apart that ${ }_{\text {skim milk bot- }}^{\text {double-n e } \mathrm{k} \mathrm{ed}}$ the small fat column can be easily esti- tle (sometimes mated to single hundredths of one per son or B. \& W.

\footnotetext{
1 See Wis. exp. station, report 17, p. 81 .

2 First constructed by Mr. J. J. Nussbaumer, of Illinois; now manufactured by various firms.
} 
cent. In the first forms, now out of use, the neck was graduated to hundredths of one per ccnt.

The value of the divisions of the scale on the doublenecked test bottles has been a subject of considerable discussion, and various opinions have been expressed whether they show one-tenth or one-twentieth (.05) of one per cent. of fat. By calibration with mercury the value of the divisions will be found to be .05 , or onetwentieth, of cne per cent., but as shown above, the results obtained in using the bottles for separator skim. milk generally come at least .05 per cent. too low, so that, practically speaking, each division may be taken to show one-tenth of one per cent., if the fat fills only one division of the scale or less. ${ }^{1}$

The double-necled bottle is very convenient for the testing of separator skim milk, thin butter milk and whey. The milk, acid and water are added to the bottle through the filling-tube; the mixing of milk and acid must be done with great care, so that none of the contents is forced into the fine measuring tube and lost; it is best to add half of the acid first and mix it with the milk, and then add the rest. When the fat is in the lower end of the measuring tube, it can be forced into the scale by pressing with the finger on the top of the side tube.

In placing the double-necked bottles in the tester they should be put with the filling tube toward the center, so as to avoid any of the fat being caught between this tube and the side of the bottle when it resumes a vertical position.

1 Wis. exp. station, bull. 52 ; Penna. exp. station, report 1896, p. 221. 
This test bottle is more fragile and expensive than the ordinary Babcock bottles, and must be carefully handled; it is now generally made of heavier glass and this form is to be highly recommended. ${ }^{1}$

100. The double-size skim milk bottle, which was the first one recommended for the testing of skim milk, is of no particular value. It is difficult to obtain a thorough mixture of the milk and the acid in these bottles, and the tests invariably come too low, more so than with the regular Babcock bottles or the double-necked skim milk bottles, for reasons that are readily seen.

ror. Buttermilk and whey. The testing of buttermilk or whey by the Babcock test offers no special difficulties, and what has been said in regard to tests of separator skim milk is equally true in case of these by-products. Whey contains only a small quantity of solids not fat, viz., less than 7 per cent. (27), and the mixing with acid and the solution of the whey solids therein is therefore readily accomplished; the acid solution is of a light reddish color, turning black but very slowly.

102. Butter. Butter is not so easily

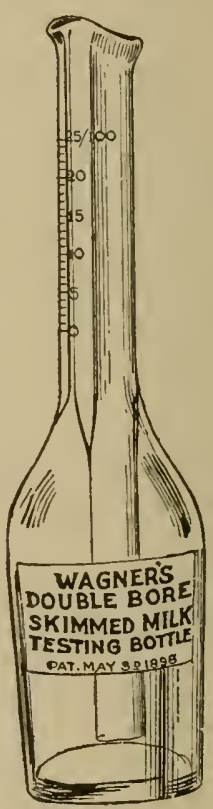

FIG. 38.

The double-bore skim milk bottle.

tested as other dairy products, both because of the difficulties in obtaining a fair sample, and on account of the high percentage of fat it contains. Butter is a mechanical mixture of water, curd, and salt, with butter-

${ }^{1}$ A double-necked copper test bottle with a detachable graduated glass neck was designed and tried by one of us a few years ago, but it was not found to possess any special advantage over the glass bottle. 
fat; and the water or brine is so easily pressed out that great care must be taken to get the same amount of water in the small portion to be tested as exists in the lot of butter sampled.

Sampling butter. Small portions of butter are taken with a butter trier or a knife from different parts of the tub, package, or churning of butter to be tested. These small portions (preferably about 200 grams in all) are placed in a wide-necked bottle or jar which is securely stoppered and placed in warm water until the butter melts. The jar is then shaken vigorously in order to obtain a thorough mixing of the various components of the butter, and placed in cold water. As the butter cools, the jar must be shaken repeatedly until the butter either solidifies or becomes of a thick creamy consistency. From this sample small portions may be taken for testing.

It is not always necessary to prepare a sample of butter for testing in the manner described. If the butter contains no loose drops of brine on the freshly-cut surface, a sample for testing can be taken directly from the package. The operator must use his judgment in regard to the necessity of preparing a special sample in each case.

Scales for weighing butter. In testing butter it is necessary to weigh the amount taken for a test very accurately. Scales sensitive to less than .05 gram should be used, as a difference of .1 gram in weight has a value of 1.0 per cent. in the result when 10 grams of butter are tested. Slow-working scales with rusted bearings are worthless for testing butter. The scales should 
always be balanced before being used and the weights kept bright and clean.

Carelessness in weighing may be the cause of very inaccurate results, and the importance of a sensitive scale cannot be over-estimated. Scales with a graduated side beam are preferable to those that require the use of small weights. Scales sensitive to .01 gram are now on the market, which permit of 20 to 50 grams of butter being weighed out for testing. ${ }^{1}$

103. Fat in butter. The Babcock test can be used with a fair degree of accuracy for estimating the per cent. of fat in butter, by weighing 9 grams of butter into a test bottle graduated to measure 50 per cent. fat. About 10 ce. of hot water is added to the butter, and 17.5 cc. of sulfuric acid of one-half the strength used in milk testing. Mix the butter and acid until the curd is all dissolved, add hot water to bring the fat into the neck of the test bottle and whirl in a centrifuge. When a clear separation of the fat is obtained the test bottle is placed in water of $140^{\circ} \mathrm{F}$. up to near the top of the neck and after standing a few minutes in this water the fat column is read off; the reading multiplied by 2 gives the per cent. of fat.

Accurate results can only be obtained by taking great care in all the manipulations, especially in weighing the butter and in reading the fat at the proper temperature. Small errors in making tests have a marked influence on the results, because the butter fat is such a large per cent. of the butter. Tests should always be made in duplicate. ${ }^{2}$

\footnotetext{
${ }^{1}$ See bull. 154, Wisconsin exp. sta., p. $1 v$.

2 Special bottles for testing butter for its fat content have been put
} 
104. Cheese. Cheese can be easily tested by the Babcock test if a small scale (fig. 34) is at hand for weighing the sample; the results obtained will furnish accurate information as to the amount of fat in the cheese, provided good judgment and exactness are used in sampling and weighing the cheese. The following method of sampling cheese is recommended. ${ }^{1}$

"Where the cheese can be cut, a narrow wedge reaching from the edge to the center of the cheese will more nearly represent the average composition of the cheese than any other sample. This may be cut quite fine, with care to avoid evaporation of water, and the portion for analysis taken from the mixed mass. When the sample is taken with a cheese trier, a plug taken perpendicular to the surface, one-third of the distance from the edge to the center of the cheese, will more nearly represent the average composition than any other. The plug should either reach entirely through or only half way through the cheese.

"For inspection purposes the rind may be rejected, but for investigations, where the absolute quantity of fat in the cheese is required, the rind should be included in the sample. It is well, when admissible, to take two or three plugs on different sides of the cheese and after splitting them lengthwise with a sharp knife, take portions of each for the test."

I05. When a satisfactory sample of the cheese has been obtained, about 5 grams are weighed into a milk test bottle or a larger quantity (say 9 grams) may be used with a cream test bottle. The test bottle is first weighed empty, and again after the pieces of cheese have been added. About 15 cc. of hot water is added to the cheese in the test bottle, and this is shaken occasionally until the cheese softens and forms a creamy emulsion

on the market, e. g., the Wagner Butter Test Bottle and the form suggested by H. R. Wright, given in the 18 th report of the Iowa State Dairy Commissioner, 1904 , p. 40.

1 U. S. Dept. of Agriculture, Chemical Division, bull. 46, p. 37. 
with the water. A few cc of acid will aid in this mixing and disintegration, the process being hastened by placing the bottles in tepid water. When all lumps of cheese have disappeared in the liquid, the full amount of acid is added, and the test completed in the ordinary manner. ${ }^{1}$

The per cent. of fat in the cheese is obtained by multiplying the reading of the fat column by 18 and dividing the product by the weight of cheese. 'The weighing of the cheese and the reading of the fat must be done very carefully, since any error introduced is more than trebled in calculating the per cent. of fat in the cheese.

ro6. Condensed milk. The per cent. of fat in unsweetened condensed milk can be obtained by weighing about 9 grams into a test bottle and proceeding in exactly the same way as given under testing of cheese. It is not necessary to warm the condensed milk in the test bottles, since this is readily dissolved in water. Enough water should ke added to make the total volume of liquid in the bottles 15 to $18 \mathrm{cc}$.

If a scale is not available for weighing the sample, fairly accurate results may be obtained by diluting the condensed milk with water $(1: 3)$, and completing the test in the ordinary manner. When this is done, the results must be corrected for the dilution which the sample received.

Hunziker ${ }^{2}$ recommends adding hot, dilute sulfuric acid solution after the first whirling, in the analysis of sweetened condensed milk (sulfuric acid and water,

${ }^{1}$ See also Sammis, Journ. Ind. and Eng. Chem., I, p. 604.

2 Ind. expt. sta., bull. 134. 
$1: 1$ ). He finds that this aids in giving a clear separation of the fat and obtaining satisfactory results.

ro7. Sweetened condensed milk. The testing of sweetened condensed milk presents peculiar difficulties, whether it is to be tested by the Babcock test or by chemical analysis. It may, however, be readily tested by the Babcock test by introducing certain changes in the manipulation of the test, as worked out by one of us. $^{1}$ A brief description of the method of analysis adopted is here given.

About sixty grams of condensed milk are weighed into a 200 cc graduated flask, to this 100 cc. of water are added and the solution of the condensed milk effected. The flask is then filled to the mark with water and after mixing thoroughly, a 17.6 cc. pipette full is measured into a Babcock test bottle. About three cc. of the sulfuric acid commonly used for testing milk are then added and the milk and acid mixed by shaking the bottle vigorously. The milk is curdled by the acid, and the curd and whey separated somewhat. In order to make this separation complete and to compact the curd into a firm lump, the test bottle is whirled for about six minutes at a rather high speed (1,000 rev.) in a steamheated turbine tester.

The chamber in which the bottles are whirled ought to be heated to abcut $200^{\circ} \mathrm{F}$. This can be done either by the turbine exhaust steam which leaks into the testbottle chamber of some machines, or by means of a valve and pipe which will allow steam to be turned di. rectly into the test bottle chamber. After this first

${ }^{1}$ Wis. exp. station, report XVII, pp. 86-89. 
whirling the bottles are taken from the tester and by being careful not to break the lump of curd nearly all the whey or sugar solution can be poured out of the neck. Ten cc. of water are then poured into the test bottle and the curd is shaken up with it so as to wash out more of the sugar. Three cc. of acid are now added as before and the test bottle whirled a second time. The whey is again decanted and this second washing removes so much of the sugar that what remains will not interfere with testing in the usual way. The curd remaining in the bottle after the second washing is shaken up with ten cc. of water; the water-emulsion of the curd is then cooled; the usual amount, 17.5 cc., of sulfuric acid is added, and the test completed in the same way as when milk is tested. The amount of fat obtained in the neck of the test bottle is calculated to the weight of condensed milk taken. ${ }^{1}$

ro8. Ice cream. Methods for determining the per cent. of fat in ice cream have recently been worked out by $\mathrm{Holm}^{2}$ and Howard. ${ }^{3}$ The former recommends the use of a mixture of equal parts of hydrochloric and glacial acetic acid, in the place of sulfuric acid, as the latter is likely to char the sugar in the ice cream, thus giving difficulty in reading the results. Nine grams of either the frozen or melted sample are weighed into a Babcock milk bottle, which is then filled almost to the neck with the mixture of the two acids given. This is heated for a few minutes until black, when the bottle is

1 The Gottlieb method gives very satisfactory results with both cheese and condensed milk (see 254).

2 Report Dept. of Health, City of Chicago, 1906, p. 50.

sourn. Am. Chem. Soc., 1907, p. 16. 
whirled in the tester and water added to bring the fat column within the graduations of the neck, as in the regular Babcock test. The reading multiplied by two gives the per cent. of fat in the ice cream.

\section{Questions.}

1. Why is it difficult to get accurate tests of skim milk by the Babcock test?

2. Mention at least three precautions that should be taken in testing skim milk.

3. Should more acid be used for full milk than for skim milk, or more for skim milk than for whey? Why?

4. How much fat is probably present in a sample of skim milk which shows no fat on being tested in a skim milk bottle?

5. What per cent. of fat does each division of a doublenecked skimmilk test bottle represent?

6. How can (a) butter, (b) cheese, (c) unsweetened and sweetened condensed milk be tested with the Babcock test?

7. If $8.4 \mathrm{gr}$. cheese give a reading of $12.2 \%$ on the neck of a test bottle, what per cent. of fat does the cheese contain?

8. What is the per cent. of fat in a sample of cheese, of which 4.2 grams contained enough fat to fill the space in the neck of a Babcock milk test bottle from 1.7 to 9.5 mark

9. How can the per cent. of fat in ice cream be determined? 
CHAPTER VI.

\section{THE LACTOMETER AND ITS APPLICATION.}

109. The lactometer is used for determining the specific gravity of milk. The term specific gravity means the weight of a certain volume of a solid or a liquid substance compared with the weight of the same volume of water at $4^{\circ} \mathrm{C}$. $\left(39.2^{\circ}\right.$ Fahr. $)$; for gases the standard of comparison is air or hydrogen. If the milk which a can will hold weighs exactly $103.2 \mathrm{tbs}$., this can will hold a smaller weight of water, say $100 \mathrm{lbs}$., as milk is heavier than water; the specific gravity of this milk will then be $\frac{130.2}{100}=1.032$.

The specific gravity of normal cow's milk will vary in different samples between 1.029 and 1.035 at $60^{\circ} \mathrm{F}$., the average being about 1.032 . The specific gravity of skim milk is about 1.036-1.038, and of sweet cream 1.01 to .95 , according to the per cent. of fat contained therein; average specific gravity 1.0 (see p. 77). ${ }^{1}$

The lactometer enables us to determine rapidly the relative weight of milk and water. Its application rests on well-known laws of physics: When a body floats in a liquid, the weight of the amount of liquid which it replaces is equal to the weight of the body. It will sink further into a light liquid than into a heavy one, be-

\footnotetext{
${ }^{1}$ Since one gallon of milk weighs 8.34 lbs., 1 gal. of milk will weigh $8.34 \times 1.032$ or $8.6 \mathrm{lbs}$.; 1 gal. of skim milk, $8.7 \mathrm{lbs}$, and 1 gal. of cream from 8 to 8.4 lbs., according to its richness.
} 
cause a larger volume of the former will be required to equal the weight of the body. A lactometer will therefore sink deeper into milk of a low specific gravity than into milk of a high specific gravity.

rro. The Quevenne lactometer. This instrument (fig. 39), consists of a hollow cylinder weighted by means of mercury or fine shot so that it will float in milk in an upright position, and provided with a narrow stem at its upper end, inside of which is found a graduated paper scale. In the better forms, like the Quevenne lactometer shown in the figure, a thermometer is melted into the cylinder, with its bulb at the lower end of the lactometer and its stem rising above the lactometer scale.

The scale of the Quevenne lactometer is marked at 15 and 40 , and divided into 25 equal parts, with figures at each five divisions of the scale. The single divisions are called degrees. The 15-degree mark is placed at the point to which the lactometer will sink when lowered into a liquid of a specific gravity of 1.015 , and the 40-degree mark at the point to which it will sink when placed in a liquid of a specific gravity of 1.040 .

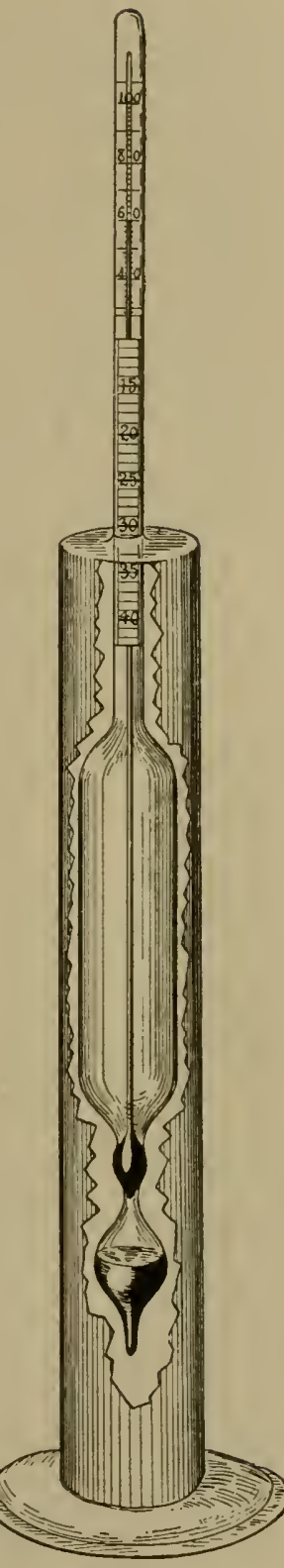

FIG. 39.

Quevenne lactometer floating in milk in a tin cylinder (115). 
The specific gravity is changed to lactometer degrees by multiplying by 1000 and subtracting 1000 from the product.

EXAMPLE: Given, the specific gravity of a sample of milk, 1.0345; corresponding lactometer degree, $1.0345 \times 1000-1000=$ 34.5 .

Conversely, if the lactometer degree is known, the corresponding specific gravity is found by dividing by 1000 and adding 1 to the quotient $(34.5 \div 1000=.0345$; $.0345+1=1.0345)$.

IIx. Influence of temperature. Like most liquids, milk will expand on being warmed, and the same volume will, therefore, weigh less when warm than before; that is, its specific gravity will be decreased. It follows then that a lactometer is only correct for the temperature at which it is standardized. If a lactometer sinks to the 32-mark in a sample of milk of a temperature of $60^{\circ} \mathrm{F}$., it will only sink to, say 33 , if the temperature of the milk is $50^{\circ} \mathrm{F}$., and will sink farther down, e. g., to 31 , if the temperature is $70^{\circ} \mathrm{F}$. Lactometers are generally standardized at $60^{\circ} \mathrm{F}$., and to show the correct specific gravity the milk to be tested should first be warmed (or cooled, as the case may be) to exactly $60^{\circ}$ F. As this is a somewhat slow process, tables have been constructed for correcting the results for errors due to difference in temperature.

II2. As the fat content of a sample of milk has a marked influence on its specific gravity at different temperatures, the co-efficient of expansion of fat differing greatly from that of the milk serum, the table can- 
not give absolutely accurate corrections for all kinds of milk, whether rich or poor. But the error introduced by the use of one table for all kinds of whole milk within a comparatively small range of temperature, like ten degrees above or below $60^{\circ}$, is too small to have any importance outside of exact scientific work, and in such, the specific gravity is always determined by means of a picnometer or a specific-gravity bottle (248), at the exact temperature at which this has been calibrated. In taking the specific gravity of a sample of milk by means of a lactometer, the milk is always warmed or cooled so that its temperature does not vary ten degrees either way from $60^{\circ} \mathrm{F}$.

I13. The temperature correction table for whole milk, given in the Appendix shows that if, e. g., the specific gravity of a sample of milk taken at $68^{\circ} \mathrm{F}$. was found to be 1.034 , its specific gravity would be 1.0352 if the milk was cooled down to $60^{\circ}$. If the specific gravity given was found at a temperature of $51^{\circ}$, the corrected specific gravity of the milk would be 1.0329 .

In practical work in factories or at the farm, suffciently accurate temperature corrections may generally be made by adding .1 to the lactometer reading for each degree above $60^{\circ} \mathrm{F}$., and subtracting .1 for each degree below $60^{\circ}$; e. g., if the reading at $64^{\circ}$ is 29.5 , it will be about $29.5+.4=29.9$ at $60^{\circ} \mathrm{F}$.; and 34.0 at $52^{\circ} \mathrm{F}$. will be about $34.0-.8=33.2$ at $60^{\circ} \mathrm{F}$. The table in the Appendix gives 33.0 as the corrected figure in both cases.

The scale of the thermometer in the lactometer should be placed above the lactometer scale so that the tem- 
perature may be read without taking the lactometer out of the milk; this will give more correct results, and will facilitate the reading.

114. N. Y. Board of Health lactometer. In the East, and among city milk inspectors generally, the so-called New York Board of Health lactometer is often used. This does not give the specific gravity of the milk directly, as is the case with the Quevenne lactometer; but the scale is divided into 120 equal parts, known as Board of Health degrees, the mark 100 being placed at the point to which the lactometer sinks when lowered into milk of a specific gravity of 1.029 (at $60^{\circ} \mathrm{F}$.) ; this is considered the lowest limit for the specific gravity of normal cow's milk. The zero mark on the scale shows the point to which the lactometer will sink in water; the distance between these two marks is divided into 100 equal parts, and the scale is contin-. ued below the 100 mark to 120 . As $100^{\circ}$ on the Board of Health lactometer corresponds to $29^{\circ}$ on the Quevenne lactometer, the zero mark showing in either case a specific gravity of 1 , the degrees on the former lactometer may easily be changed into Quevenne lactometer degrees by multiplying by .29. To further aid in this transposition, Table III is given in the Appen$d i x$, showing the readings of the two scales between $60^{\circ}$ and $120^{\circ}$ on the Board of Health lactometer.

The temperature correction for Board of Health lactometers is as follows: for each degree of temperature above $60^{\circ} \mathrm{F}$. .3 is added to the reading, and for each degree below, .3 is subtracted.

II5. Reading the lactometer. For determining the specific gravity of milk in factories or private dairies, tin or copper cylinders, $11 / 2$ inches in diameter and 10 inches high, with a base about four inches in diameter, are recommended (see fig. 39); another form of specificgravity cylinders, in use in chemical laboratories, is shown in fig. 40. The cylinder is filled with milk of a temperature ranging between $50^{\circ}$ and $70^{\circ} \mathrm{F}$., to within an inch of the top, and the lactometer is slowly lowered therein until it floats; it is left in the milk for about 
half a minute before lactometer and thermometer readings are taken, both to allow the escape of air which has been mixed with the milk in pouring it, preparatory to the specific-gravity determination, and to allow the thermometer to adjust itself to the temperature of the milk. The lactometer should not be left in the milk more than a minute before the reading is taken, as cream will soon begin to rise on the milk, and the reading, if taken later, will be too high, as the bulb of the lactometer will then be floating in partially skimmed milk (23). In reading the lactometer degree, the mark on the scale plainly visible through the upper portion of the meniscus of the milk should be noted. Owing to surface tension the milk in immediate contact with the lactometer stem will rise above the level of the surface in the cylinder,

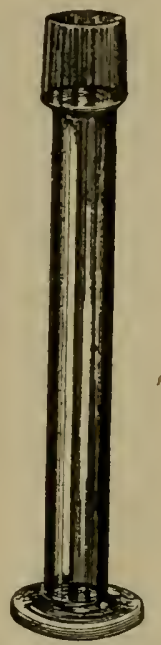

Fig. 40. Specific-gravity and this must be taken into consideration in taking the readings. It is not necessary to read closer than onehalf of a lactometer degree in factory or dairy work.

Ir6. Time of taking lactometer readings. The specific gravity of milk should not be determined until an hour or two after the milk has been drawn from the udder, as too low results are otherwise obtained (Recknagel's phenomenon). ${ }^{1}$ The cause of this phenomenou is not definitely understocd; it may come from the escape of gases in the milk, or from changes occurring in the mechanical condition of the nitrogenous compo-

\footnotetext{
1 Milchztg. 1883, 419 ; bull. 43, Chem. Div., U. S. Dept. of Agriculture, p. 191 ; Analyst, 1894 , p. 76 .
} 
nents of the milk. The results obtained after a couple of hours will, as a rule, come about one degree higher than when the milk is cooled down directly after milking and its specific gravity then determined.

x17. Influence of solid preservatives on lactometer readings. When potassium bi-chromate, corrosive sublimate, etc., is added to milk samples to preserve them from souring (190), the specific gravity of the milk will be increased; with the quantity usually added ( $1 / 2$ gram to a pint of milk) the increase amounts to about 1 lactometer degree, and this correction of lactometer readings should be made with milk samples preserved in this manner. To avoid this error, Dr. Eichloff ${ }^{1}$ recommends the use of a solution of potassium bichromate in water (43 grams to 1 liter), the specific gravity of which is 1.032 , or similar to that of average milk; 5 cc. of this solution is required for a pint of milk. No correction is necessary for the dilution with this small amount of liquid preservative.

II8. Cleaning the lactometer. The lactometer should be cleaned directly after using, by rinsing with cold water; it is then wiped dry with a clean cloth and placed in the case.

II8a. Testing the accuracy of lactometers. The correctness of lactometers may be determined with a fair degree of accuracy by placing them in different salt solutions prepared by dissolving exactly 3,4 , and 5 grams of pure dairy salt in 100 grams (cc.) of water. The specific gravities at $60^{\circ} \mathrm{F}$. of solutions thus ob-

1 Technik der Milchfrüfung, p. 9S. 
tained are 1.022, 1.029, and 1.036, for 3,4 , and 5 per cent. solutions, respectively.

\section{Calculation of Milk Solids.}

119. A number of chemists have prepared formulas for the calculation of milk solids when the fat content and the specific gravity (lactometer reading) of the milk are known. By careful work with milk tester and lactometer it is possible by means of these formulas to determine the composition of samples of milk with considerable accuracy, both outside of and in chemical laboratories. As the complete formulas given by various chemists (Behrend and Morgen, Clausnitzer and Mayer, Fleischmann, Hehner and Richmond, Richmond, Babcock $)^{1}$ are very involved, and require rather lengthy calculations, tables facilitating the figuring have been prepared. The formulas in use at the present time, in this country and abroad, are those proposed by Fleischmann, Hehner and Richmond, or Babcock. Babcock's formula is the one generally taught in American dairy schools and is therefore given here; it forms the foundation for Table VI in the Appendix for calculation of solids not fat.

By the use of these tables the percents of solids not fat may be found, corresponding to lactometer readings from 26 to 36 , and to fat contents from 0 to 6 per cent. The formula, as amended in $1895,{ }^{2}$ is as follows, $S$ being the specific gravity and $f$ the per cent. of fat in the milk.

$$
\text { Solids not } \mathrm{fat}=\left(\frac{100 \mathrm{~S}-\mathrm{Sf}}{100-1.0753 \mathrm{Sf}}-1\right)(100-\mathrm{f}) 2.5
$$

1 Agricultural Sclence, vol. III, p. 139.

${ }^{2}$ Wisconsin experiment station, twelfth report, page 120 . 
The derivation of this formula is explained in the report referred to.

I20. Short formulas. The tables made up from this formula, giving the percentages of solids not fat corresponding to certain per cents. of fat and lactometer readings, are given in the Appendix. A careful examination of the tables will disclose the fact that the per cent. of solids not fat increases uniformly at the rate of .25 , or one-fourth of a per cent. for each lactometer degree, and .02 per cent. for each tenth of a per cent. of fat. This relation is expressed by the following simple formulas :

\section{Solids not $\mathrm{fat}=1 / 4 \mathrm{~L}+.2 \mathrm{f}$ \\ Total solids $=1 / 4 \mathrm{~L}+1.2 \mathrm{f}$,}

$L$ being the lactometer reading at $60^{\circ} \mathrm{F}$. (specific gravity $\times 1000-1000$ ), and $f$ the per cent. of fat in the milk.

Rule: a, To find the per cent. of solids not fat in milk, add two-tenths of the per cent. of fat to one-fourth of the lactometer reading, and

$\mathrm{b}$, To find the per cent. of total solids in milk, add one and two-tenths times the per cent. of fat to one-fourth of the lactometer reading.

These formulas and rules are easily remembered and can be quickly applied without the use of tables. The results obtained by using them do not differ more than .04 per cent. from those of the complete formula for milks containing up to 6 per cent. of fat, and may be safely applied in practical work.

The English dairy chemist Droop Richmond has constructed an ingenious sliding "milk scale" which enables one to readily find the percentages of total solids. 
corresponding to different lactometer readings and fat contents, or the percentage of fat from total solids and lactometer readings. ${ }^{1}$

\section{Adulteration of Milk.}

I21. Methods of adulteration. The problem of determining whether or not a sample of milk is adulterated becomes an important one in the work of milk inspectors and food chemists. Nanagers of creameries and cheese factories are also sometimes interested in ascertaining pcssible adulterations in the case of some patron's milk, although since the general introduction of the Babcock test in factories and the payment for the milk $\mathrm{cn}$ the basis of the amount of butter fat delivered, the temptation to water or skim the milk has been largely removed. In the city milk trade, especially in our larger cities, watered or skimmed milk is still often met with, in spite of the vigilance of their milk inspectors or the officers of the city boards of health.

When the origin of a suspected sample of milk is known, a second sample should always be taken on the premises, if possible, by or in the presence of the inspector, and the composition of the two samples compared. If the suspected sample is considerably lower in fat content than the second, so-called control-sample, and has a normal per cent. of solids not fat, it is skimmed; if the solids not fat are below normal, it is watered; and if both these percentages are abnormally low, the sample is most likely both watered and skimmed (126).

\footnotetext{
${ }^{1}$ Dairy Chemistry, p. 61.
} 
r22. Latitude of variation. In order to determine whether or not a sample of milk is skimmed or watered, or both skimmed and watered, the per cents. of fat and of solids not fat in the sample must be ascertained, and if a control-sample can be secured, these determinations for both samples compared. The proper latitude to be allowed for the natural variation in the composition of milk differs according to the origin of the milk; in case of milk from single cows, the variations in fat content from day to day may exceed one per cent., although under ordinary conditions the per cent. of fat in most cow's milk will not vary that much. The content of solids not fat is more constant, and rarely varies one-half of one per cent. from day to day with single cows. Cows in heat or sick cows may give milk differing considerably in composition from normal milk. ${ }^{1}$

123. Mixed herd milk is of comparatively uniform composition on consecutive days, and as most milk offered for sale or delivered to factories is of this kind, the task of the milk inspector is made considerably easier and more certain on this account. Daily variations in herd milk beyond one per cent. of fat and onehalf per cent. of solids not fat, are suspicious and may be taken as fairly conclusive evidence of adulteration. This is especially true in case the control-sample shows a comparatively low content of fat or solids not fat (159).

r24. Legal standards. Where a control-sample cannot be taken, the legal standards of the various states for fat or solids in milk are used as a basis for calculat-

\footnotetext{
${ }^{1}$ Blythe, Foods, their Composition and Analysis, London, 1903, p. 250 et seq.
} 
ing the extent of adulteration of a sample of milk. A list of legal standards for milk in this country and abroad is given in the Appendix. These standards determine the limits below which the milk offered for sale within the respective states must not fall. Legally it matters not whether a sample of milk offered for sale has been skimmed or watered by the dealer or by the cow; in the latter case, the cows producing the milk are of a breed or a strain that has been bred persistently for quantity of milk, without regard to its quality. In most states the legal standard for the fat content of milk is 3 per cent., and for solids not fat 9 per cent. There are, however, cows which at times produce milk containing only 2.5 to 2.8 per cent. of fat, and less than 8.5 per cent. solids not fat. Such milk cannot therefore be legally sold in most states in the Union, and the farmer offering such milk for sale, even if he does not know the composition of the milk produced by his cows is liable to prosecution just as if he had directly watered the milk. By mixing the milk of several cows, the chances are that the mixed milk will contain more fat and solids not fat than called for by the legal standard; if such should not be the case, cows producing richer milk must be added to the herd so as to raise the quality of the herd milk up to the legal standard, or the cows giving very thin milk must be disposed of.

125. The specific gravity of the milk solids. A calculation of the specific gravity of the milk solids is of considerable assistance in interpreting the results of analyses of suspected milk samples. The milk solids vary but slightly in specific gravity, viz., between 1.25 and 1.34 , the richer milks having sol- 
ids of low specific gravities. The specific gravity of the milk solids is calculated by means of Fleischmann's formula

$$
S=\frac{t}{t-\frac{100 s-100}{s}}
$$

$S$ being the sp. gr. of the milk solids, $s$ that of the milk and $t$ the total solids of the milk.

Example: A sample of milk has been found to contain 13.0 per cent. of solids, sp. gr. 1.032; then $\frac{100 \times 1.032-100}{1.032}=3.101$; $13.0-3.101=9.899 ; \frac{1}{9.3 .0}=1.31$, the specific gravity of the milk solids.

The specific gravity of the solids does not change if the milk is watered, while it is increased when the milk is skimmed. If a sample of milk of the composition given in the preceding example had been watered so as to reduce the solids to 11.7 per cent. and the specific gravity to 1.0291 (as would be the case when 10 per cent. of water was added), we would again have, by calculation as above, $\mathrm{S}=1.31$. If, on the other hand, the milk was skimmed so as to reduce the solids to 11.7 per cent., thereby increasing the specific gravity of the milk to, say 1.035, we would have by substituting these values in the preceding formula, $\mathrm{S}=1.41$, showing conclusively that the milk had been skimmed.

An addition of skim milk to whole milk would have the same effect as skimming, as regards the composition of the latter, and the specific gravity of its solids.

The specific gravity of pure butter fat at $60^{\circ} \mathrm{F}$. is .93 , and of the fat-free milk solids, 1.5847 (Fleischmann). The solids of skim milk have a specific gravity of 1.56. Samples of whole milk, the solids of which have a specific gravity above 1.34 are suspicious, and a specific gravity over 1.40 is conclusive evidence of skimming.

To facilitate the calculation of the specific gravity of milk solids, Table IV is given in the Appendix, showing at a glance the value of $\frac{100 \mathrm{~s}-100}{\mathrm{~s}}$ for specific gravities between 1.019 and 1.0369. An example will readily illustrate the use of the table.

Example: A sample of milk has a specific gravity of 1.0343 and contains 12.25 per cent. solids. In Table IV, we find in the 
norizontal line beginning with 1.034 under the column headed 0.0003 , the figure 3.316 , which is the value for $\frac{100 s-100}{s}$ wen $\mathrm{s}=1.0343$. Introducing this value and that of the total solids in the formula, the calculation is $12.25-3.316=8.934 ; 12.25 \div$ $8.934=1.37$, which is the specific gravity of the solids in this case.

r26. To recapitulate. Adulteration of milk by watering or skimming, or both, may be established by a comparison of the composition of the suspected sample with that of a control-sample, or if none such can be obtained, with the legal standards. If the compcsition of the two samples varies appreciably, the milk has been adulterated, and the character of the aduiteration is shown from the following statement:

If the analysis of the suspected sample shous

the milk is

sp. gr. of milk
fat and solids not fat........ low

sp. gr. of solids normal

sp. gr. of milk and of solids_._._._.
solids not fat.....

fat and soliuls -low

sp. gr. of milk normal

sp. gr. of solids ormal or high \{

fat and solids not fat'.......... low

watered

skimmerl watered and skimmed

The extent of the adulteration is determined as given below.

127. Calculation of extent of adulteration. ${ }^{1}$ In the following formulas, percentages found in the controlsamples, if such are at hand, are always substituted for the legal standards.

a. Skimming.-1. If a sample of milk has been skimmed, the following formula will give the number of pounds of fat abstracted from $100 \mathrm{lbs}$. of milk:

\footnotetext{
${ }^{1}$ Woll, Handbook for Farmers and Dairymen, New York, 1907, pp. $\because 67-8$
} 
Fat abstracted=legal standard for fat-f, . .

$f$ being the per cent. of fat in the suspected sample.

2. The following formula will give the per cent. of fat abstracted, calculated on the total quantity of fat originally found in the milk:

$$
x=100-\frac{f \times 100}{\text { legal standard for fat }} \quad-\quad-
$$

b. Watering.-If a sample is watered, the calculation is most conveniently based on the percentage of solids not fat in the milk. The percentage adulteration may be expressed either on basis of the amount of water present in the adulterated milk, or the amount of water added to the original milk:

1. Per cent. of foreign (extraneous) water in the adulterated milk $=100-\frac{\mathrm{S} \times 100}{\text { legal standard for solids not fat }}$ (III) $\mathrm{S}$ being the per cent. of solids not fat in the suspected sample.

Example: A sample of milk contains 7.5 per cent. solids not fat; if the legal standard for solids not fat is 9 per cent., then $100-\frac{7.5 \times 100}{9}=16.7$, shows the per cent. of extraneous water in the milk.

2. Watering of milk may also be expressed in per cent. of water added to the original milk, by formula IV :

Per cent. of water added to the original milk $=\frac{100 \times \text { leg. stand. for sol. not fat }}{\mathrm{S}}-100$

In the example given above, $\frac{100 \times 9}{7.5}-100=20$ per cent. of water was added to the original milk.

c. Watering and skimming. - If a sample has been both watered and skimmed, the extent of watering is 
ascertained by means of formula (III) or (IV), and the fat abstracted found according to the following formula:

Per cent. fat abstracted $=$

leg. stand. for fat $-\frac{\text { leg. stand. for sol. not fat }}{\mathrm{S}} \times f$. .

Example: A sample of milk contains 2.4 per cent. of fat and 8.1 per cent. solids not fat; then

Extraneous water in milk $=100-\frac{8 \cdot 1 \times 100}{9}=10$ per cent.

Fat abstracted $=3-\frac{9 \times 24}{8.1}=33$ per cent.

$100 \mathrm{lbs}$. of the milk contained $10 \mathrm{lbs}$. of extraneous water and $.33 \mathrm{lb}$. of fat had been skimmed from it.

For methods of detection of other adulterations and of preservatives in dairy products, see Chap. X, 299, et seq.

\section{Questions.}

1. What is the weight of 1000 cc. of (a) water; (b) skim milk; (c) whole milk; (d) cream testing $30 \%$ fat; (e) whey; (f) butter fat?

2. If the sp. gr. of a sample of milk is 1.0325 at $68^{\circ} \mathrm{F}$., what is the lactometer reading at $60^{\circ}$

3.' What effect on the sp. gr. has $1.0 \%$ solids not fat and $1.0 \%$ fat?

4. How can the accuracy of a lactometer be determined

5. If a sample of milk has a sp. gr. of 1.032 and $13.0 \%$ solids, what is the sp. gr. of the milk solids?

6. How can (a) skimmed milk, (b) watered milk, (c) skimmed and watered milk bo detected 9

7. Give lactometer readings and percentages of fat in sam. ples showing (a) watering, (b) skimming, (c) watering and skimming.

8. If one quart of water is added to one quart of milk, what per cent. of water is added, and what per cent. extraneous water does the mixture contain 
9. How many pounds of fat have been removed from 100 pounds of a sample of milk testing $2.6 \%$, and what per cent. of the fat was removed?

10. If a sample of milk contains $7.0 \%$ solids not fat, what per cent. water was added and how much extraneous water did the sample contain?

11. What has probably been done to each of the following samples of milk, that were found to contain (a) 7.2 per cent. solids not fat, 2.6 per cent. fat; (b) 9.0 per cent. solids not fat, 2.5 per cent. fat; (c) 6.5 per cent. solids not fat, $2.4 \%$ fat?

12. What is the per cent. solids not fat and what is the condition of each of the following samples of milk:

\section{Lactometer Reading.}

(a) 32.0 at $58^{\circ} \mathrm{F}$.

(b) 33.5 at $56^{\circ} \mathrm{F}$.

(c) 30.0 at $63^{\circ} \mathrm{F}$.

(d) 28.0 at $54^{\circ} \mathrm{F}$.

(e) 27.4 at $69^{\circ}$.F.
Per Cent Fat.

4.0

2.5

3.5

2.5

2.4 


\section{TESTING THE ACIDITY OF MILK AND CREAM.}

128. Cause of acidity in milk. Even directly after milk is drawn from the udder it will be found to have an acid reaction, when phenolphtalein is used as an indicator. ${ }^{1}$ The acidity of fresh milk is not due to the presence of free organic acids in the milk, like lactic or citric acid, but to acid phosphates, and possibly also in part to free carbonic acid gas in the milk or to the acid reaction of casein. Even in case of so-called sweet milk, nearly fresh from the cow, a certain amount of acidity, viz., on the average about .07 per cent., is therefore found. When the milk is received at the factory it will rarely test less than .10 per cent. of acid, calculated as lactid acid; some patrons bring milk day after day that does not test over .15 per cent. of acid; that of others tests from .20 to .25 per cent., and some lots, although very rarely, will test as high as .3 of one per cent. of acid. It has been found that milk will not usually smell or taste sour or "turned," until it contains .30 to .35 per cent. of acid.

129. The acidity in excess of that found normally in milk as drawn from the udder, is due to other causes than those described. Bacteriological examinations of milk from different sources and of milk of the same origin at different times have shown that there is, roughly speaking, a direct relation between the bacteria found

\footnotetext{
${ }^{1}$ Freshly drawn milk shows an amphoteric reaction to litmus, i. e., it colors blue litmus paper red, and red litmus paper faintly blue.
} 
in normal milk, and its acidity; the larger the number of bacteria per unit of milk, the higher is, in general, the acidity of the milk. The increase in the acidity of milk on standing is caused by the breakingdown of milk sugar in ito lactic acid through the activities of acid-forming bacteria. Since the bacteria get into the milk through a lack of cleanliness during the milking, or careless handling of the milk after the milking, or both, it follows that an acidity test of new milk will give a good clue to the care bestowed in handling the milk. Such tests will show which patrons take good care of their milk and which do not wash their cans clean, or their hands and the udders of the cows before milking, and have, in general, dirty ways in milking and caring for the milk. The acidity test is always higher in summer than in winter, and is generally high in the case of milk kept for more than a day (Monday milk), or delivered after a warm, sultry day or night. The bacteria have had a good chance to multiply greatly in such milk, even if it be kept cooled down to $40^{\circ}-50^{\circ} \mathrm{F}$., and as a result considerable quantities of lactic acid have been formed. The determination of the acidity of fresh milk is explained in detail below (147).

r30. Method of testing acidity. Methods of measuring the acidity or alkalinity of liquids by means of certain chemicals giving characteristic color reactions in the presence of acid or alkaline solutions (so-called volumetric methods of analysis) have been in use for many years in chemical laboratories. They were applied to milk as early as 1872 by Soxhlet, ${ }^{1}$ and the method

\footnotetext{
1 Jour. f. prakt. Chemie, 1872, p. 6, 19.
} 
worked out by Soxhlet and Henkel has since been in general use by European chemists. They measured out 50 cc. of milk to which was added 2 cc. of a 2 per cent. alcoholic solution of phenolphtalein, and this was titrated with a one-fourth normal soda solution ${ }^{1}$ (see below). In this country, Dr. A. G. Manns in 1890 published the results of work done in the line of testing the acidity of milk and cream, ${ }^{2}$ and the method of procedure and apparatus proposed by him has become known under the name of Manns' test, and is being advertised as such by dealers in dairy supplies.

13I. Manns' test. The acid in milk or cream is measured by using an alkali solution of a certain strength, with an indicator which shows by a change of color in the milk when all its acid has been neutralized. Any of the alkalies, soda, potash, ammonia, or lime can be used for making the standard solution, but it requires the skill and apparatus of a chemist to prepare it of the proper strength. A tenth-normal solution ${ }^{3}$ of caustic soda is the alkali solution used most frequently in de-

${ }^{1}$ Fleischmann, Lehrb. d. Milchwirtschaft, 3rd ed., p. 57.

2 Illinois experiment station, bulletin 9 .

${ }^{3}$ Normal solutions, as a general rule, are prepared so that one liter shall contain the hydrogen equivalent of the active reagent weighed in grams (Sutton). Caustic soda $(\mathrm{NaOH}$, is made up of an atom each of sodium (Na), oxygen $(\mathrm{O})$, and hydrogen $(\mathrm{H})$; its molecular weight is therefore

$$
23+16+1=40
$$

$\mathrm{NaOH}$

A normal soda solution then is made by dissolving 40 grams of soda in water, making up the volume to $1000 \mathrm{cs}$ : a one-tenth normal solution will contain one-tenth of this amount of soda, or 4 grams dissolved in one liter. One cubic centimeter of the latter solution will contain .004 gram of soda, and will neutralize .009 gram of lactic acid. The formula for lactic acid is $\mathrm{C}_{3} \mathrm{H}_{6} \mathrm{O}_{2}$ (see p. 16), and its molecular weight is therefore $3 \times 12+6 \times 1+3 \times 16=90$. A tenth-normal solution of lactic acid contains 9 grams per liter, and .009 gram per cubic centimeter. 
termining the acidity of milk, and is the solution labeled Neutralizer of the Manns' test.

The indicator used is a solution of phenolphtalein, a light yellowish powder; its compounds with alkalies are red, in weak alkaline solutions pink colored, while its acid compounds are colorless. The phenolphtalein solution used is prepared by dissolving 10 grams in $300 \mathrm{cc}$. of 90 per cent. alcohol (Mohr).

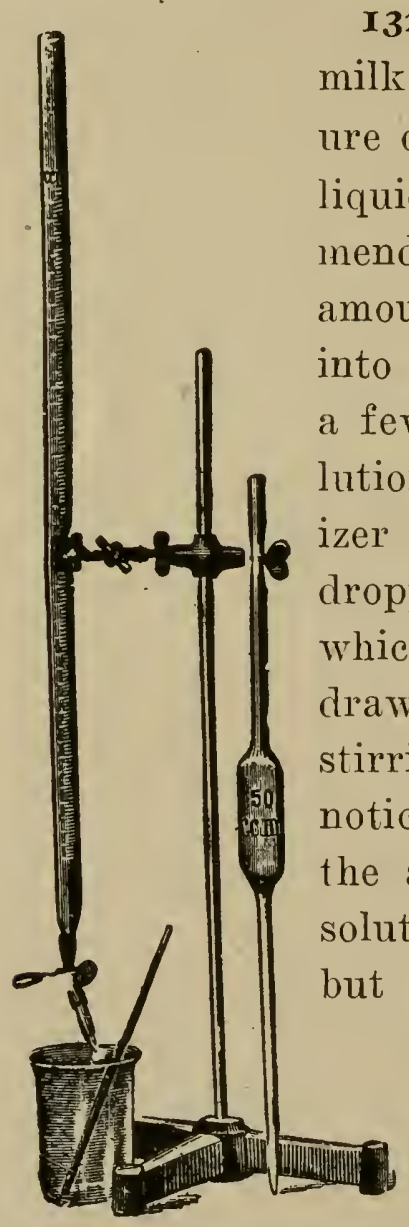

Fig. 41. Apparatus used in Manns' test.

132. In testing the acidity of either milk or cream it is necessary to measure out with exactness the quantity of liquid to be tested; Manns recommended using a 50 cc. pipette. This mount of milk or cream is measured to a clean tin, porcelain or glass cup, few drops of the phenolphtalein solution are added, and the Neutralizer (or alkali solution) is cautiously dropped in from a burette, the point at which the solution stands before any is drawn off being noted. By constant stirring during this operation it will be noticed that the pink color formed by the addition of even a drop of alkali solution will at first entirely disappear, but as more and more of the acid in the sample becomes neutralized, the color will disappear more slowly, until finally a point is reached when the pink color remains permanent for a time. No 
more alkali should be added after the first appearance of a uniform pink color in the sample. This color will fade and gradually disappear again on standing, owing to the effect of the carbonic acid of the air, to which phenolphtalein is very sensitive. The amount of the alkali solution used for the test is then obtained from the reading on the scale of the burette. The per cent. of acid in the sample is calculated by multiplying the number of ce. of alkali solution used, by .009 and dividing the product by the number of cc. of the sample tested, the quotient being multiplied by 100 .

$$
\text { Per cent. acidity }=\frac{\text { c. c. alkali } \times .009}{\text { c. c. } \text { sample tested }} \times 100
$$

If 50 cc. of cream required 32 cc. of alkali solution to produce a permanent pink color, the per cent. of acid in the cream would be $\frac{32 \times .009}{50} \times 100=.58$ per cent. A part of this calculation may be saved by using a factor for multiplying the number of cc. of alkali added in each test. This factor is obtained by dividing .009 (the number of grams of lactic acid neutralized by one cc. of alkali solution) by the number of cc. of sample tested, and multiplying the quotient by 100 . If a 50 cc. pipette is used for measuring the sample to be tested, the factor will be $(.009 \div 50) \times 100=.018$; if a 25 cc. pipette is used, the factor will be $(.009 \div 25) \times 100=$ .036 ; and if a 20 cc. pipette is used, $(.009 \div 20) \times 100=$ .045 will be the factor to be applied in calculating the per cent. of acidity, the number of cc. of alkali used being in all cases multiplied by the particular factor corresponding to the volume of the sample tested. 
133. If a Babcock milk test pipette is used for measuring the milk or cream to be tested for acidity, the factor will be $(.009 \div 17.6) \times 100=.051$. This is so nearly .05 that sufficiently accurate results may be obtained by simply dividing the number of cc. used by two; the result will be the tenths of per cent. of acid in the sample tested, e. g., if 17.6 cc. of cream required 12 cc. of onetenth normal alkali to give a pink color, then the per cent. of acid is $12 \div 2=.6$ per cent. If one-fifth normal alkali is used for testing, the per cent. of acidity is shown directly by the number of cc. used (Vivian). ${ }^{1}$

134. Manns' testing 'outfit. The apparatus (see fig. 41) and chemicals necessary for testing the acidity of milk or cream by the so-called Manns' test include one gallon of a one-tenth normal alkali solution; four ounces of an alcoholic solution of phenolphtalein, a 50 cc. glass burette provided with a pinchcock, a burette stand and a pipette for measuring the sample. This outfit will make about 100 tests and is sold for $\$ 5.00 .^{2}$

I35. The alkaline tablet test. Solid alkaline tablets were proposed by Farrington in 1894, as a substitute for the liquid used in Manns' test. ${ }^{3}$ It was found possible to mix a solid alkali carbonate and coloring matter, and compress the mixture into a small tablet, which would contain an exact amount of alkali. The advantage of the tablets lies in the fact that they

1 Van Norman recommends the use of a 50th normal solution for testing cream (see Purdue exp. sta., bull 104). 37 cc. or a normal soda solution is diluted to $1850 \mathrm{cc}$. in a two-quart bottle, such as is used for mineral waters. Each cc. of this solution represents .01 cc. of acidity when $1 . .6 \mathrm{cc}$. of cream is measured off. The titration is made in the usual manner, using phenolphtalein as an indicator.

${ }^{2}$ Devarda's acidimeter (Milchzeitung 1896, p. 785) is based on the same principle as Manns' test; one-tenth soda solution is added to 100 cc. of milk in a glass-stoppered granulated flask, $\angle$ cc. of a 4 per cent. phenolphtalein solution being used as an indicator. The graduations on the neck of the flask give the "degrees acidity" directly.

'Illinois experiment station, bulletin 32, April, 1894. 
will keep far better than a standard alkali solution, and they can be safely sent by mail; they also require less apparatus and are considerably cheaper than standard alkali solutions; 1000 of these tablets, costing $\$ 2.00$, will make about 400 tests. $^{1}$ Similar alkaline tablets were placed on the market in Europe at about the same time, viz., Stokes' Acidity Pellets in 1893, and Eichler's Säurepillen (acid pills) in $1895 .^{2}$

Two methods of using the tablets have been proposed, one, for the titration (determination of acidity) of ripening cream in the manufacture of sour-cream butter: and the other, for determining the approximate acidity

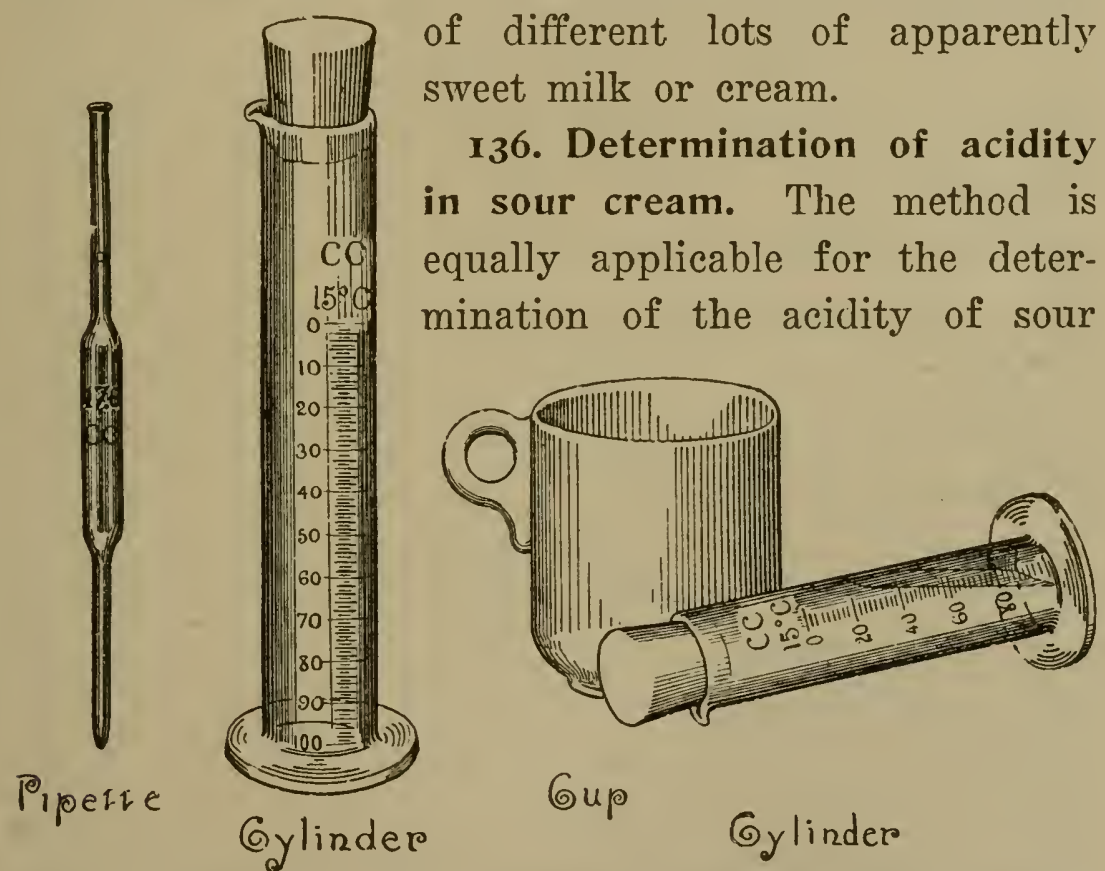

FIG. 42. Apparatus used for determining the acidity of cream or milk. cream, sour milk and buttermilk, but is most frequently employed in testing the acidity of cream, to examine

1 The tablets are sold by dealers in dairy supplies.

${ }^{2}$ Milchzeitung, 1895, pp. 513-16. 
whether or not the ripening process has reached the proper stage for churning the cream. The apparatus used (see fig. 42) is as follows:

1. 17.6 cc. pipette.

1 white cup.

100 ec. graduated cylinders; it is well to provide two or three of these, although only one is strictly necessary.

137. Preparation of the solution. The tablet solution formerly used was prepared by dissolving five tablets in 50 cc. of water; with 20 cc. of cream each cubic centimeter of this solution represents .017 per cent. of acid (lactic acid) in the sample tested. The amount of acid in a given sample is then obtained by multiplying the number of cubic centimeters of the tablet solution used, by .017 .

138. According to a suggestion made by Mr. C. L. Fitch, ${ }^{1}$ the strength of the solution was changed in such a manner that the percentages of acidity are indicated directly by the number of cubic centimeters of tablet solution used in each test.

The 17.6 cc. pipette may be used for measuring the sample for acidity testing, and the results read directly from the graduated cylinder, if the tablet solution is prepared by taking one tablet for every 19.5 cc. of water; five tablets are therefore dissolved in 97 cc. of water.

139. As eream during its ripening process under the conditions present in this country generally has from .5 to .6 per cent. of acid before it is ready to be churned,

\footnotetext{
${ }^{1}$ Hoard's Dairyman, Sept. 3, 1897.
} 
a 50 cc. cylinderful of tablet solution of this strength will not be sufficient to make a test of cream containing over .5 per cent. of acid, although it is enough for testing the cream up to this point during the ripening process. The acid-testing outfit should therefore contain a 100 cc. graduated cylinder, instead of one of 50 cc. capacity, so that cream of any amount of acidity up to 1 per cent. can be tested. A tablet solution of the strength given has not only the advantage over the solution previously recommended ( 5 tablets to 50 ce. of water) ${ }^{1}$ of showing the per cent. of acidity directly, without tables or calculations, but being weaker, the unavoidable errors of determination are decreased by its use.

Since a 17.6 cc. pipette is found in creameries and dairies with the Babcock test outfit, no new apparatus is necessary for making the acidity test in the manner given.

140. The preparation of the standard solution is as follows: Five tablets are placed in the 100 cc. cylinder which is filled to the $97 \mathrm{cc}$. mark with clean soft water. ${ }^{2}$ The cylinder is tightly corked, shaken and laid on its side, as the tablets dissolve more quickly when the cylinder is placed in this position than when left upright with the tablets at the bottom. Several cylinders containing the tablet solution may be prepared at a time; as soon as one is emptied, tablets and water are again added, and the cylinder is corked and placed in a hori-

\footnotetext{
${ }^{1}$ Illinois experiment station, bulletin 32 ; Wisconsin experiment station, bulletin 52 .

2 Condensed steam or rain water should be used, and not hard or alkali water, since the impurities in these affect the strength of the tablet solution,
} 
zontal position. In this way fresh solutions ready for testing are always at hand. The cylinder is kept tightly corked while the tablets are dissolving, so that none of the liquid is lost by the shaking. It is well to put the tablets in the cylinder with water at night; the solution will then be ready for use in the morning. Excepting a flocculent residue of inert matter, "settlings," which will not dissolve, the tablets must all disappear in the solution before this is used. The strength of the tablet solution does not change perceptibly by standing, at least for one week. The only precaution necessary is to avoid evaporation of the solution by keeping the cylinders tightly corked. The solid tablets will not change if kept dry, any more than dry salt changes by age.

I4r. Accuracy of the tablets. The tablets have been repeatedly tested by chemists and found to be accurate and uniform in composition. Tests made with the tablets according to the directions here given can therefore be relied on as correct. The alkali solution is very sensitive, however, and should not be measured in a cylinder which has been previously used for measuring sulfuric acid, as the smallest drop or film of acid from a dish or from the operator's fingers will change the strength of the standard tablet solution.

Powdered sodium carbonate weighed out exactly in the quantity required for making a gallon of tenth normal solution has of late been placed on the market; these "test powders" are cheaper than alkaline tablets and when put out by a reliable firm are equally as accurate as these. 
142. Making the test. The cream to be tested is thoroughly mixed, and $17.6 \mathrm{cc}$. are measured into the cup. The pipette is rinsed once with water, and the rinsings added to the cream in the cup. A few cc. of the tablet solution prepared as given above are now poured from the cylinder into the cream and mixed thoroughly with it by giving the cup a gentle rotary motion. The tablet solution is added in small quantities until a permanent pink color appears in the sample. The number of cc. of tablet solution which has been used to color the cream is now read off on the scale of the cylinder.

In comparing the results of one test with another, the same shade of color should always be adopted. ${ }^{1}$ The most delicate point is the first change from pure white or cream color to a uniform pink which the sample shows when the acid contained therein has been neutralized. This shade of color is easily recognized with a little practice. The pink color is not permanent unless a large excess of the alkaline solution has been added, on account of the influence of the carbonic acid of the air (132), and the operator should not therefore be led to believe by the reappearance of the white color after a time, that the point of neutralization was not already reached when the first uniform shade of pink was observed.

143. Acidity of cream. 17.6 cc. of sweet cream is

${ }_{1}$ A helpful suggestion has been made by the Danish State Dairy lnktructor, Dr. (;. Ellbrecht, for obtaining a uniform color in acidity tests. Strips of pink paper are attached to the cul or glass in which the titration is made, and alkali solution is added, until the color of the milk or cream corresponds to that of the strips. 
generally neutralized by 15 to 20 ce. of this tablet solution, representing from .15 to .20 per cent. of acid. A mildly sour cream is colored by $35 \mathrm{cc}$. tablet solution, and a sour cream ready for churning by about 50 to 60 ce. tablet solution. As the eream ripens, its acidity increases. The rate of ripening depends largely on the temperature at which the cream is kept. Cream containing .5 to .6 per cent. of acid will make such butter as the general American market demands at the present time. Cream showing an acid test of .55 per cent. may not be too sour, but .65 per cent. of acid is very near, if not on the danger line, since such cream is likely to make strong flavored, almost rancid butter. Each lot of cream should be tested as soon as it is ready for ripening, and the result of the test will show whether the cream should 'ue warmed or cooled in order to have it ready for churning at the time desired. Later tests will show the rate at which the ripening is progressing, and the time when the eream has reached the proper acidity for churning.

r44. The influence of the richness of cream on the acid test has been studied by Professor Spillman, ${ }^{1}$ and others. ${ }^{2}$ Since the acidity develops in the cream serum, it follows that an acidity of, say .5 per cent. in a 40 per cent. cream represents a larger acidity than in 20 per cent. of cream, e. g.; in the former case we have .5 gram of acid in 60 grams of serum ( $=83$ per cent. of the serum); in the latter case .5 gram acid is found in 80 grams serum (=.63 per cent. of the serum). There-

\footnotetext{
1 Washington experiment station, bulletin 32 .

${ }^{2}$ Chicago Dairy Produce, April 21, 1900, p. 30 ; Iowa expt. sta., bull. 52.
} 
fore, rich cream need not be ripened to as high a degree of acidity as thin cream. A table is given in the Iowa bulletin referred to, showing the relation between the richness and the acidity of cream.

145. Spillman's cylinder. The graduated cylinder shown in fig. 43 was devised by Professor Spillman for use in testing the acidity of milk and cream with Farrington's alkaline tablets. The following directions are given for making tests with this piece of apparatus: ${ }^{1}$

"All that is needed in addition to the acid-test graduate shown in the accompanying illustration, is a common prescription bottle of six or eight ounce capacity, and a package of Farrington's alkaline tablets. Fill the bottle with water and add one tablet for each ounce of water in the bottle. Shake the bottle frequently to aid in dissolving the tablets.

"Making the test. In making the test, the acid-test graduate is filled to the zero mark with the milk or cream to be tested. The tablet solution is then added, a little at a time, and the graduate shaken after each addition, in order to thoroughly mix the milk and the tablet solution. In shaking the graduate, give it a rotary motion to prevent spilling any of the liquid. adding the tablet solution until a permanent pink used in $\begin{gathered}\text { uning the } \\ \text { macid- }\end{gathered}$ color can be detected in the milk. The level of ity of cream or the liquid in the graduate, measured by the scale on the graduate, will then show the per cent. of the acidity of the milk. It is best to stand the graduate on a piece of white paper, so that the first pink coloration of the milk may be easily detected."

146. The Marschall acid test (see fig. 44) is a convenient apparatus for determining the acidity of milk, cream, or whey. ${ }^{2}$ It is used with a tenth-normal alkaline

1 Washington experiment station, bulltin 24 .

${ }^{2}$ See Wis. exp. sta., bull. 129. 
solution ("Neutralizer"), 9 cc. of milk, cream, etc., being measured out for the test, and alkali solution added from the combined burette and bottle, the former being graduated to two-tenths of one cc. The burette is filled by tipping the bottle and the surplus of the "neutralizer" will flow back, leaving the solution at

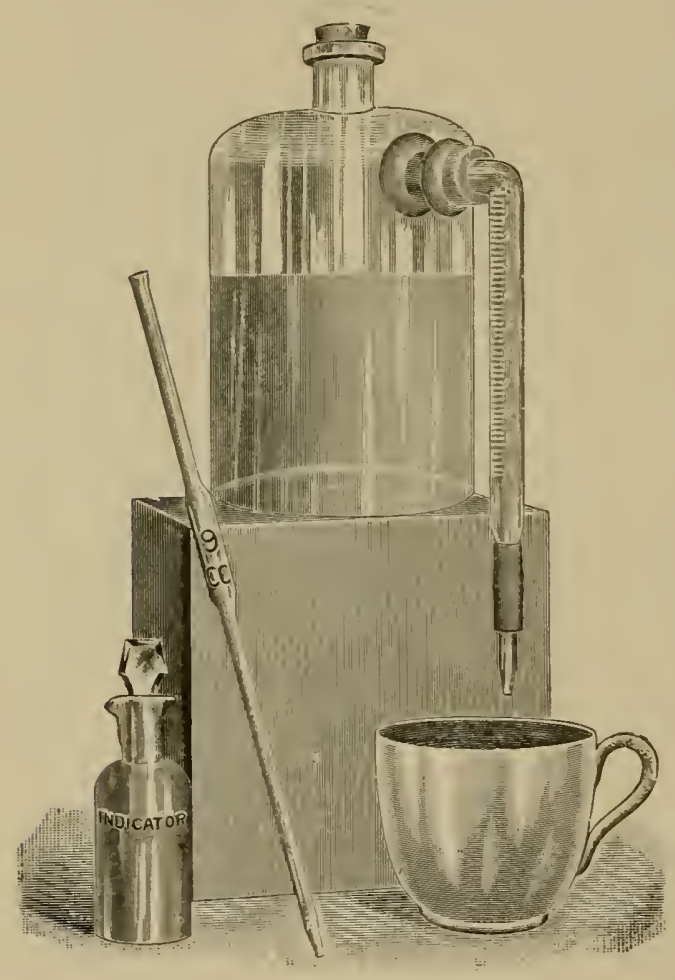

FIG. 44. The Marschall acid test. the zero mark. With the quantity of milk given, the readings obtained represent per cent of acidity direct.

147. Rapid estimation of the acidity of apparently sweet milk or cream. a, Milk. The alkaline tablet method offers a ready means of estimating the acidity of milk or cream that is still sweet to the taste. The selection of the best linds of milk is especially important in pasteurizing milk or cream. As previously noted, milk which gives the highest acid test contains, as a rule, a larger number of bacteria and spores not destroyed by pasteurization than does milk giving a low acid test (129); the acidity test may therefore be used to advantage for the purpose of selecting milk best adapted for pasterrization, 
as well as such as is to be retailed or used in the manufacture of high-grade butter and cheese.

In distinguishing milk fit for pasteurization purposes from that which is doubtful, an arbitrary standard of two-tenths of one per cent. of acid may be taken as the
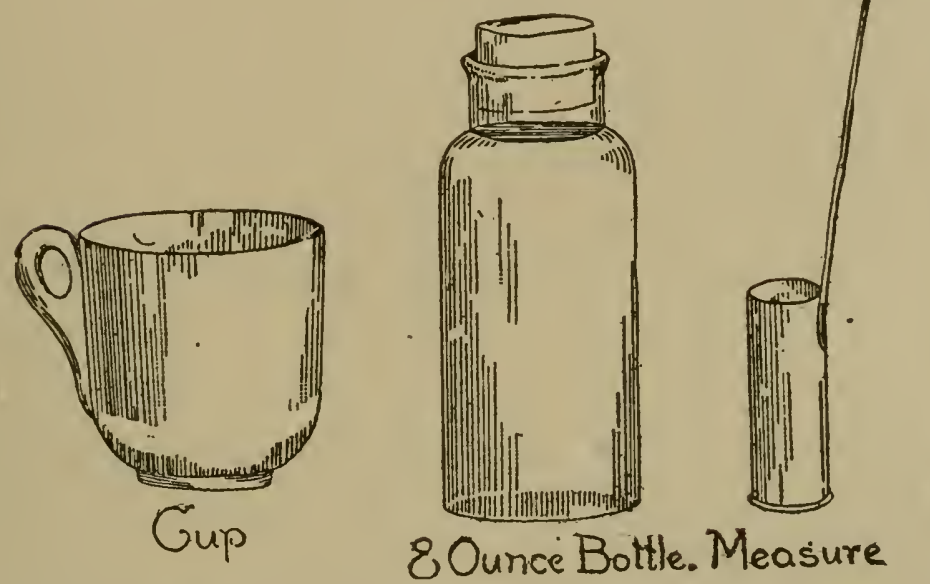

Fig. 45. Apparatus used for rapid estamation of the acidity of ap. parently sweet milk ol cream.

upper limit for milk of the former kind. The apparatus used in making this test is shown in the accompanying illustration (fig. 45), and consists of a white teacup; a four-, six-, or eight-ounce bottle, and a No. 10 brass cartridge shell, or a similar measure. A solution of the tablets in water is first prepared, one tablet being always added to each ounce of water: four tablets in a four-ounce bottle; six, in a six-ounce bottle, etc., the amount of tablet solution prepared depending on the 
number of tests to be made at a time. The bottle is filled up to its neck with clean, soft water, and the solution prepared in the manner previously given (140).

I48. Operating the test. As each lot of milk is brought to the creamery in the morning and poured into the weigh can, a cartridge-shell dipper is filled with milk and this is poured into the white cup. The same or another No. 10 shell is now filled twice with the tablet solution and emptied into the milk in the cup. Instead of dipping twice with one measure or a No. 10 shell, a tin measure can be make holding as much as two No. 10 shells, or the tablet solution may be made of double strength; that is, two tablets to each ounce of water and the same sized measure used for both the milk and the tablet solution. The liquids are then mixed in the cup by giving this a quick, rotary motion, and the color of the mixture noticed. If the milk remains white it contains more than two-tenths of one per cent. of acid and should not be used for pasteurization. If it is colored after having been thoroughly mixed with two - measures of tablet solution, it contains less than this amount of acid and may, as far as acidity goes, be safely used for pasteurization or for any other purpose which requires thoroughly sweet milk. The shade of color obtained will vary with different lots of milk; the sweetest milk will be most highly colored, but a milk retaining even a faint pink color with two measures of tablet solution, or one measure of the double strength solution to one measure of milk, contains less than .2 per cent. of acid. 
By proceeding in the manner described, the man receiving and inspecting the milk at the weigh-can is able to test the acidity of the milk delivered nearly as quickly as he can weigh it; and according to the results of the test he can send the milk to the general delivery vat or to the pasteurization vat, as the weigh-can may be provided with two conductor spouts.

149. Size of measure necessary. It is not necessary to use a No. 10 shell for a measure in working the preceding method; one of any convenient size that can be filled accurately and quickly, will answer the purpose equally well, if a measure of the same size is used for both the sample and the tablet solution. Each measureful of tablet solution made up as directed, will in this case represent one-tenth per cent. of acid in the sample tested. ${ }^{1}$

I50. b, Cream. Cream can be tested in the way already described for testing the acidity of fresh milk, by adding to one measureful of cream in the cup as many measures of tablet solution as are necessary to change the color of the cream when the two liquids are thoroughly mixed. If one measure of tablet solution colors one measure of cream, this contains less than .1 per cent. acid; if five measures of tablet solution are required, the cream contains about .5 per cent. acid, etc. By proceeding in the manner described, the operator can estimate the acidity to within .05 per cent. of acid, if half measures of tablet solution are added. The re-

${ }^{1}$ In European creameries and city milk depots the alcohol test is often applied to every can of muk received; milk that is sufficiently sour to be noticed by the taste, will coagulate when mixed with an equal volume of $70 \%$ alcohol. 
sults thus obtained are sufficiently delicate for all practical purposes.

151. Detection of boracic-acid preservatives in milk. The application of the alkaline tablet test for detecting the boracic acid in milk was first discussed in bulletin No. 52 of Wisconsin experiment station. The acidity of the milk is increased by the addition of boracic acid, but neither the odor nor the taste of the milk is affected thereby. By adding to sweet milk the amount of boracic acid which will keep it sweet 36 hours, its acidity may be increased to .35 per cent., in a sample of milk which previously tested perhaps only .15 per cent. acid.

As before stated, unadulterated milk will usually smell or taste sour or "turned," when it contains $.30-.35$ per cent. acid (121); milk testing as high as this limit, which neither smells nor tastes sour in any way, is therefore in all probability adulterated with some preparation containing boracic acid or a similar compound.

152. "Alkaline tabs." These are not the alkaline tablets, but a substitute which was put on the market by a New York firm. The outfit furnished consisted of four packages of paper dises made of heavy filter pajer, each of about the size of an oldstyle copper cent; two packages of square paper; one glass of abont 10 cc. capacity, and one small glass bottle. An investigation of these "Tabs" soon disclosed the fact that they were entirely inaccurate, and that no dependence could therefore be placed on the results obtained by their use.

\section{Questions.}

1. If 20 ce. eream require 12 ce. $\frac{N}{10}$ alkali for neutralization, what per cent. acid in the sample?

2. If 1 cc. ${ }_{10}^{N}$ alkali nentralize .009 gram lactic acid, what is the per cent. of acid in a sample of cream, which required 12 cc. alkali for 25 cc. of cream?

3. What apparatus and strength of solution must be taken to show per cent. acidity directly from cc. alkali used with Far rington's alkaline tablets?

4. If cream testing $20 \%$ fat has an acidity of $.6 \%$, what will be the corresponding acidity of cream testing $40 \%$ fat? 


\section{CHAPTER VIII.}

\section{TESTING THE PURITY OF MILK.}

153. The Wisconsin curd test. Cheese makers are often troubled with so-called floating or gassy curds which produce cheese defective in flavor and texture. These faults are usually caused by some particular lot of milk containing impurities that cannot be detected by ordinary means of inspection. The Wisconsin curd test is used to detect the source of these defects and thus enable the cheese maker to exclude the milk from the particular farm or cow to which the trouble is traced. This test is similar in principle to tests that have long been in use in cheese-making districts in Europe, notably in Switzerland, ${ }^{1}$ but was worked out independently at the Wisconsin Dairy school in 1895 and is now generally known as the "Wisconsin Curd T'est.' "'

154. Method of making the test. Pint glass jars, thoroughly cleaned and sterilized with live steam, are provided; they are plainly numbered or tagged, one jar being provided for each lot of milk to be tested. The jars are filled about two-thirds full with milk from the varions sources; it is not necessary to take an exact

${ }^{1}$ II erz, Unters, d. Kuhmilch, Berlin, 1889. p. S7 ; Siats, Unters. landw. wicht. Stoffe, 1!0:3, p. 140.

2 Wisconsin experiment station, twelfth report, p. 14s. The apparatus used. for the test was greatly improved in 189S, and a description of the imploved test is given in bulletin No. 67 and the annual report of the Station for 18 s. (fiftecnth report. pl. $47-5.8$ ), from which source the acompanying, illustration is taken (see fig. 46). 
quantity; they are then placed in a water tank, the water of which is heated until the milk in the jars has a temperature of $98^{\circ} \mathrm{F}$. In transferring the thermometer used from one jar to another, special care must be taken to clean it each time in order to prevent contamination of pure lots of milk by impure ones.

When the milk has reached a temperature of $98^{\circ}$, add to each sample ten drops of rennet extract, and mix by giving the jar a rotary motion. The milk is thus curdled, and the curd allowed to stand for about twenty

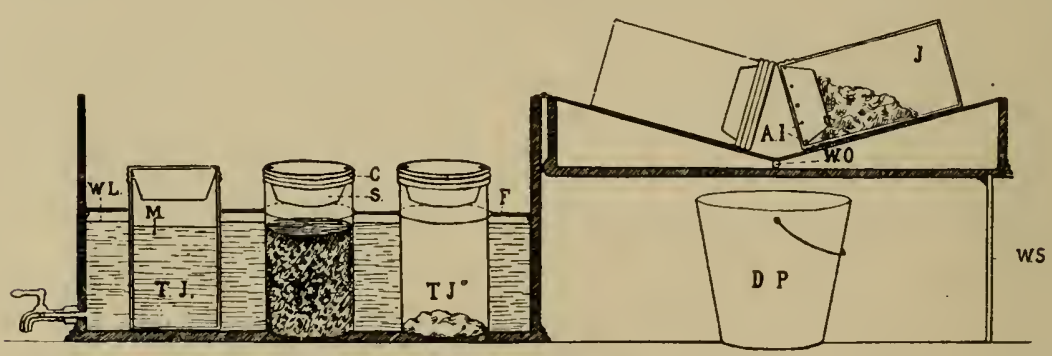

F1G. 46. Cross-section of the Wisconsin curd test, T.J-TJ". testing jars showing different stages of test; Wh, water line; M, milk; $F$, frame; WS, stand to support cover; AI, drain holes; WO, water outlet; DP, drain pail.

minutes until it is firm. It is then cut fine with a case knife, and stirred at intervals for one-half to threequarters of an hour sufficiently to keep the curd from matting under the whey. When the cubes are quite firm the whey is poured off and the curd left to mat at the bottom of the bottles if the old form of apparatus is used. The best tests are made when the separation of the whey is most complete. By allowing the samples to stand for a short time, more whey can be poured off, and the curd thereby rendered firmer. The water around the jars is kept at a temperature of $98^{\circ}$, the vat is cov- 
ered, and the curds allowed to ferment in the sample jars for six to twelve hours.

During this time the impurities in any particular sample will cause gases to be developed in the curds so that by examining these, by smelling of them and cutting them with a sharp knife, those having a bad flavor, or a spongy or in any way abnormal texture may be easily detected, and thus traced to the milk causing the trouble.

Since the curd test was first described, several modifications have been made in the apparatus. In one of these the bottles are held in a covered metal frame so that all of them can be drained at once by inverting the frame.

155. By proceeding in the way described with the milk from the different cows in a herd, the mixed milk of which produced abnormal curds, the source of contamination in the herd may be located. Very often the trouble will be found to come from the cows drinking foul stagnant water or from fermenting matter in the stable. In the former case the pond or marsh must be fenced off, or the cows kept away from it in other ways; in the latter, a thorough cleaning and disinfection of the premises are required. If the milk of a single cow is the source of contamination, it must be kept by itself, until it is again normal; under such conditions the milk from the healthy cows may, of course, safely be sent to the factory.

156. The fermentation test. The Gerber fermentation test (see fig. 47) also furnishes a convenient method for examining the purity of different lots of milk. The test consists of a tin tank which can be heated by means of a small lamp, 
and into which a rack fits, holding a certain number of cylindrical glass tubes; these are all numbered and provided with a mark and a tin cover. In making the test, the tubes are filled to the mark with milk, the number of each tube being recorded in a note book, opposite the name of the patron whose milk was placed therein. The tubes in the rack are put in the tank, which is two-thirds full of water:

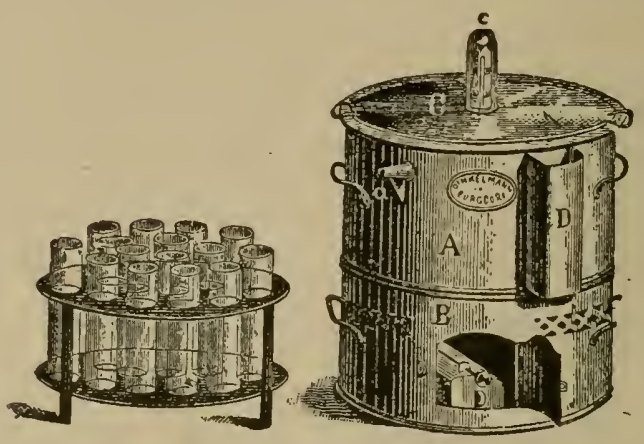

Fir. 4t. The Ferber fermentation test. the temperature of the water is kept at $104-106^{\circ} \mathrm{F}$., for six hours, when the rack is taken out, the tubes gently shaken, and the appearance of the milk, its odor, taste, etc., carefully noted in each case.

The tubes are then again heated in the tank at the same temperature as before, for another six hours, when observations of the appearence of the milk in each tube are once more taken. The tainted milk may then easily be discovered by the abnormal coagulation of the sample. According to Gerber, ${ }^{1}$ good and properly handled milk should not coagulate in less than twelve hours, when kept under the conditions described, and should not show anything abnormal when coagulated. Milk from sick cows and from cows in heat, or with diseased udders, will always coagulate in less than twelve hours. If the milk does not curdle within a day or two, it should be tested for preservatives (299).

I57. The Monrad rennet test is used by cheese makers for determining the ripeness of milk. Fig. 48 shows the apparatus used in the test. 5 cc. of rennet extract is measured into a 50 cc. flask by means of a pipette; the pipette is rinsed with water, and the flask filled to the mark with water. 160 cc. of milk is now measured into the tin basin from the cylinder and slowly heated to exactly $86^{\circ} \mathrm{F}$. 5 cc. of the dilute rennet solution is

${ }^{1}$ Dic praktische Milchpriifung, p. S5. 
then quickly added to the warm milk and the time
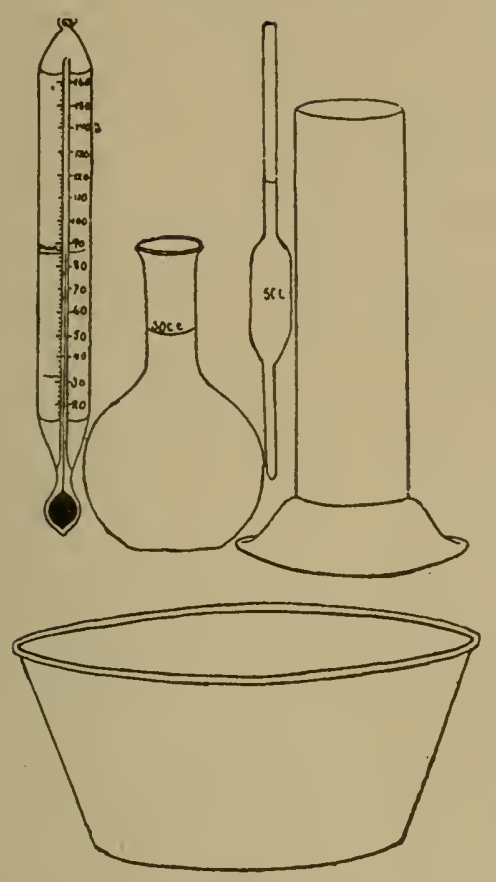
Fis. 48. The Monrad rennet test. water. Fill the cup with milk to the zero mark, add the rennet, mix thoroughly and allow it to stand. The sweeter the milk is, the longer it will take to coagulate, and the more milk will run out of the cup before the point of coagulation is reached, when the flow of milk will cease. The time required for coagulating the milk is shown directly by a scale on the

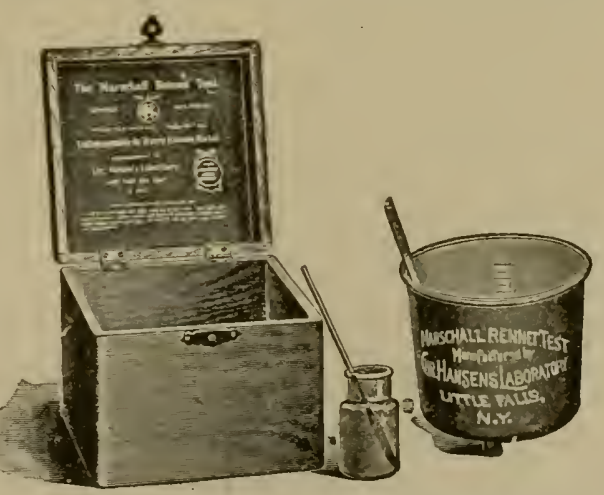

FIG. 49, The Marschall rennet test. inside wall of the cup (see fig. 49).

${ }^{1}$ Decker, Cheese Making, Revised ed., 1909, p. 39. 
CHAP'TER IX.

\section{TESTING MILK ON THE FARM.}

159. Variations in milk of single cows. The variations in the tests of milk of single cows from milking to milking or from day to day, are greater than many cow-owners suspect. There seems to be no uniformity in this variation, except that the quality of the milk produced generally improves with the progress of the period of lactation; even this may not be noticeable, however, except when the averages of a number of tests made at different stages during the lactation period are compared with each other. When a cow gives her maximum quantity of milk, shortly after calving, the quality of her milk is generally poorer (by one per cent. of fat or less) than when she is drying off. Strippers' milk is therefore, as a rule, richer in fat than the milk of fresh cows.

I60. By testing separately every milking of a number of cows through their whole period of lactation, the results obtained have seemed to warrant the following conclusions in regard to the variations in the test of the milk from single cows, and it is believed that these conclusions allow of generalization. ${ }^{1}$

1. Some cows yield milk that tests about the same at every milking, and generally give a uniform quantity of milk from day to day.

1 Illinois experiment station, bulletin 24 . 
2. Other cows give milk that varies in an unexplainable way from one milking to another. Neither the morning nor the evening milking is always the richer, and even if the interval between the two milkings is exactly the same, the quality as well as the quantity of milk produced will vary considerably. Such cows are generally of a nervous, excitable temperament, and are easily affected by changes in feed, drink, or surrounding conditions.

3. The milk of a sick cow, or of a cow in heat, as a rule, tests higher than when the cow is in normal condition; the milk yield generally decreases under such conditions; marked exceptions to this rule have, however, been observed.

4. Half-starved or underfed cows may give a small yield of milk testing higher than when the cows are properly nourished, probably on account of an accompanying feverish condition of the animal. The milk is, however, more generally of an abnormally low fat content, which may be readily increased to the normal per cent. of fat by liberal feeding.

5. Fat is the most variable constituent of milk, while the solids not fat vary within comparatively narrow limits. The summary of the analyses of more than 2400 samples of American milk calculated by Cooke ${ }^{1}$ shows that while the fat content varies from 3.07 to 6.00 per cent., that of casein and albumen varies only from 2.92 to 4.30 per cent., or less than one and one-half per cent.,

${ }^{1}$ Vermont experiment station, report for 1890 , p. 97 ; Woll's Handbook for Farmers and Dairymen, Fifth ed., p. 250. 
and the milk sugar and ash content inereases but little (about .69 per cent.) within the range given.

6. A test of only one milking may give a very erroneous impression of the average quality of a certain cow's milk. A composite sample (see 179) taken from four or more successive milkings will represent the average quality of the milk which a cow produces at the time of sampling.

I6r. The variations that may occur in testing the milk of single cows, are illustrated by the following figures obtained in an experiment made at the Illinois experiment station, ${ }^{1}$ in which the milk of each of six cows was weighed and analyzed daily during the whole period of lactation. Among the cows were pure-bred Jerseys, Shorthorns and Holsteins, the cows being from three to eight years of age and varying in weight from 850 to 1350 lbs. During a period of two months of the year, the cows were fed a heavy grain ration consisting of twelve lbs. of corn and cob meal, six lbs. of wheat bran, and six lbs. of linseed meal, per day per head. This system of feeding was tried for the purpose of increasing, if possible, the richness of the milk. The influence of this heavy grain feed, as well as that of the first pasture grass feed, on the quality and the quantity of the milk produced is shown in the following table, which gives the complete average data for one of the cows (No. 3). The records of the other cows are given in the publication referred to; they were similar to the one here given in so far as variations in quality are concerned.

${ }^{1}$ Bulletin 24. 
Average results obtained in weighing and testing a cow's milk daily during one period of lactation.

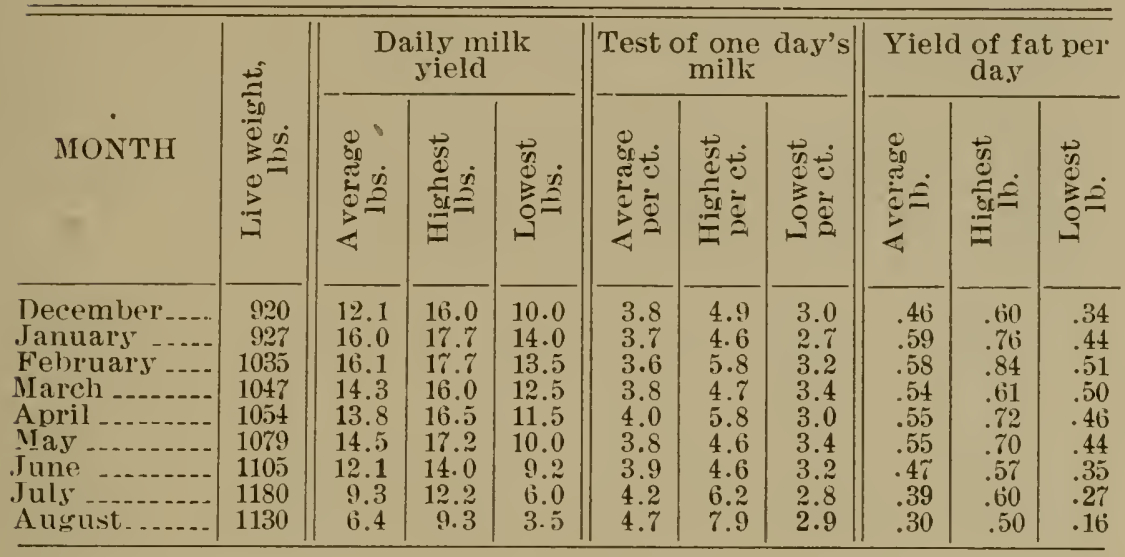

I62. The average test of this cow's milk for her whole period of lactation was 3.8 per cent. of fat (i. e., the total quantity of fat produced - total milk yield $X$ 100); twice during this time the milk of the cow tested as high as 5.8 per cent., and once as low as 2.7 per cent. 'The average weight of milk produced per day by the cow was 14 lbs.; this multiplied by her average test, 3.8 , shows that she produced on the average $.53 \mathrm{lb}$, or about one-half of a pound, of butter fat per day during her lactation period. If, however, her kutter-producing capacity had been judged by the test of her milk for one day only, this test might have been made either on the day when her milk tested 5.8 per cent., or when it was as low as 2.7 per cent. Both of these tests were made in mid-winter when the cow gave about $16 \mathrm{lbs}$. of milk a day. Multiplying this quantity by .058 gives .93 lb. of fat, and by .027 gives $.43 \mathrm{lb}$. of fat. Either 10 
result would show the butter fat produced by the cow on certain days, but neither gives a correct record of her actual average daily performance for this lactation period.

A sufficient number and variety of tests of the milk of many cows have been made to prove that there is no definite regularity in the daily variations in the richness of the milk of single cows. The only change in the quality of milk common to all cows is, as stated, the natural increase in fat content as the cows are drying off, and even in this case the improvement in the quality of the milk sometimes does not occur until the milk yield has dwindled down very materially.

163. Causes of variations in fat content. The quality of a cow's milk is, as a rule, decidedly influenced by the following conditions:

Length of interval between milkings.

Change of feed.

Change of milkers.

Rapidity of milking.

Exposure to rain or bad weather.

Rough treatment.

Unusual excitement or sickness.

164. Disturbances like. those enumerated frequently increase the richness of the milk for one, and sometimes for several milkings, but a decrease in quality follows during the gradual return to normal conditions, and taken as a whole there is a considerable falling off in the total production of milk and butter fat by the cow, on account of the nervous excitement which she has gone through. Aside from changes due to well- 
definable causes like those given above, the quality of some cows' milk will often change considerably without any apparent cause. The dairyman who is in the habit of making tests of the milk of his individual cows at regular intervals will have abundant material for study in the results obtained, and he will soon be able to tell from the tests made, if these are continued for several days, whether or not the cows are in a normal healthy condition or have been subjected to excitement or abuse in any way.

165. Number of tests required during a period of lactation in testing cows. The daily records of the six cows referred to on page 142 furnish data for comparing their total production of milk and butter fat during one period of lactation, as found from the daily weights and tests of their milk, with the total amount calculated from weights and tests made at intervals of $7,10,15$ or 30 days. The averages of all results obtained with each of the six cows show that weighing and testing the milk of a cow every seventh day gave 98 per cent. of the total milk and butter fat, which according to her daily record was the total product. Tests made once in two weeks gave 97.6 per cent. of the total milk, and 98.5 per cent. of the total butter fat, and tests made once a month, or only ten times during the period of lactation, gave 96.4 per cent. of the total milk, and 97 per cent. of the total production of butter fat.

166. The record of one of the cows will show how these calculations are made: It was found from the daily weights and tests that cow No. 1, in one lactation period of 307 days, gave 5,044 lbs. of milk which con- 
tained 254 lbs. of butter fat. Selecting every thirtieth day of her record as testing day, the total production of milk and fat is shown to be as follows:

Production of milk and butter fat per day.

\begin{tabular}{|c|c|c|c|}
\hline Testing day & Weight of Milk & Test of Milk & Yield of butter fat \\
\hline $\begin{array}{ll}\text { Nov. } & 4 \\
\text { Dec. } & 4 \\
\text { Jan. } & 3 \\
\text { Feb. } & 2 \ldots \\
\text { Mar. } & 3 \\
\text { April } & 2 \\
\text { May } & 2 \\
\text { June } 1 & 1 \\
\text { July } 1 & 1 \\
\text { July } 31 & \ldots\end{array}$ & $\begin{array}{l}\text { Lhs. } \\
20.5 \\
18.7 \\
17.7 \\
20.0 \\
18.2 \\
19.5 \\
17.7 \\
13.1 \\
12.2 \\
3.2\end{array}$ & $\begin{array}{c}\text { Per cent. } \\
4.7 \\
4.6 \\
4.9 \\
4.5 \\
4.7 \\
4.4 \\
4.8 \\
5.5 \\
6.2 \\
7.2\end{array}$ & $\begin{array}{l}\text { Lhs. } \\
.96 \\
.86 \\
.86 \\
.90 \\
.86 \\
.81 \\
.85 \\
.72 \\
.76 \\
.23\end{array}$ \\
\hline Total $\ldots \ldots$ & 160.8 lbs. & $\ldots$ & 7. 81 lbs. \\
\hline Average per day - & 16.08 lbs. & 4.85 & $.78 \mathrm{lb}$. \\
\hline
\end{tabular}

The average daily production of the cow, according to the figures given in the preceding table, was about 16 lbs. of milk, containing .78 lb. of butter fat. Multiplying these figures by 307 , the number of days during which the cow was milked, gives $4,912 \mathrm{lbs}$. of milk and $240 \mathrm{lbs}$. of fat. This is $132 \mathrm{lbs}$. of milk and $14 \mathrm{lbs}$. of fat less than the total weights of milk and butter fat, as found by the daily weights and tests, or 2.8 and 5.5 per cent. less, for milk- and fat production, respectively. This is, however, calculated from only ten single weights and tests, while it required over 600 weighings and 300 tests of the milk to obtain the exact amount.

Similar calculations from the records of the other cows gave fully as close results, showing that quite sat- 
isfactory data as to the total production of milk and butter fat of a cow may be obtained by making correct weighings and tests of her full day's milk once every thirty days.

167. When to test a cow. The Vermont experiment station for several years made a special study of the question when a cow should be tested in order to give a correct idea of the whole year's production, when only one or two tests are to be made during the lactation period. ${ }^{1}$ The results obtained may be briefly summarized as follows:

a. As to quality of milk produced. If two tests of each cow's milk are to be made during the same lactation period, it is recommended to take composite samples at the intervals given below.

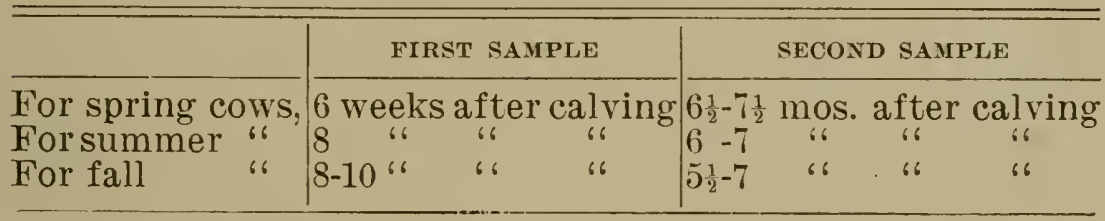

If only one test is to be made, approximately correct results may be obtained by testing the milk during the sixth month from calving, in case of spring cows; during the third to fifth month in case of summer-calving cows, and during the fifth to seventh month for fallcalving cows.

In all cases composite samples of the milk for at least two days should be taken (169). "The test of a single sample, drawn from a single milking or day, will not of necessity, or indeed usually, give trustworthy results."

${ }^{1}$ Sixth report, 1882 , p. 106 ; Ninth report, 1895 , p. 176. 
b. As to quantity of milk produced. The milk may be weighed for two days in the middle of the month, and the entire month's yield obtained with considerable

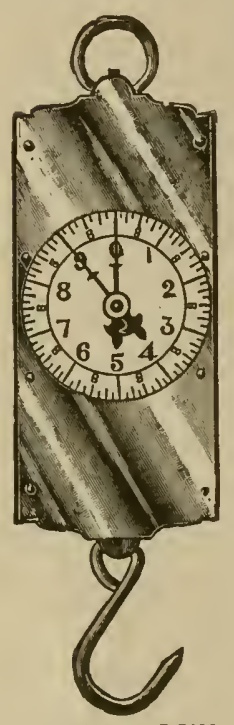

FIG. 50. Milk scale. have not proved satisfactory. ${ }^{1}$

I68. Sampling milk of single cows. In sampling the milk of single cows, all the milk obtained at the milking must be carefully mixed, by pouring it from one vessel to another a few times, or stirring it thoroughly by means of a dipper moved up and down, as well as horizontally, in the pail or can in which it is held; a sample for testing purposes is then taken at once. A correct sample of a cow's milk cannot be obtained by milking directly into a small bottle from one teat, or by filling the bottle with a little milk from each

\footnotetext{
1 The various state experiment stations now conduct official tests of dairy cows for breeders and dairy farmers, in which the production of milk and butter fat by cows is determined accurately by representatives of the stations. - Information concerning these tests may be had by writing to the director of the nearest experiment station.
} 
teat, or by taking some of the first, middle and last milk drawn from the udder. Such samples cannot possibly represent the average quality of the milk of one entire milking, since there is as much difference between the first and the last portions of a milking, as between milk and cream. ${ }^{1}$ Lack of care in taking a fair sample is the cause of many surprising results obtained in testing the milk of single cows.

169. Composite samples. When a cow is to be tested for milk production she should be milked dry the last milking previous to the day when the test is to be made. The entire quantity of milk obtained at each milking is mixed and sampled separately. On account of the variations in the composition of the milk, a number of tests of successive milkings must be made. As this involves considerable labor, the plan of taking composite samples is preferable; the method of composite sampling and testing is explained in detail under the second subdivision of Chapter X (180); suffice it here to say that the method followed in the case of single cow's or herd milk is to take about an ounce of the thoroughly mixed milk of each milking; this is placed in a pint or quart glass jar containing a small quantity of some preservative, preferably about half a gram (8 grains) of powdered potassium bi-chromate. If a number of composite samples of the milk of single cows are taken, each jar should be labeled with the number or name of the particular cow. Composite tests are generally taken for two or four days or for a week. If continued for a week, the jars will

1 Agricultural Science, 6, pp. 540-42. 
contain at the end of this time a mixture of the milk of fourteen milkings. The composite sample is then carefully mixed by pouring it gently a few times from one jar to another, and is tested in the ordinary manner. The result of this test shows the average quality of the milk produced by the cow during the time the milk was sampled.

As the amounts as well as the quality of the milk produced by single cows vary somewhat from day to day and from milking to milking, it is desirable in testing single cows, especially when the test includes only a few days, to take a proportionate part (an aliquot) of each milking for the composite test sample. This is easily done by means of a Scovell sampling tube, the use of which is explained in another place (183), or by a 25 cc. pipette divided into $\frac{1}{10} \mathrm{cc}$; in using the latter apparatus as many cubic centimeters and tenths of a cubic centimeter of milk are conveniently taken each time for the composite sample as the weight of milk in pounds and tenths of a pound produced by the cow. ${ }^{1}$

170. The opinion is sometimes expressed that a considerable error is introduced by' measuring out milk warm from the cow for the Babcock test, since milk expands on being warmed, and a too small quantity is obtained in this manner. By calculation of the expansion of milk between different temperatures it is found that 1 cc. of milk at $17.5^{\circ} \mathrm{C}$. (room temperature) will have a volume of 1.006289 cc. at $37^{\circ} \mathrm{C}$. (blood-heat), i. e., an error of less than .03 per cent. is introduced by measuring out milk of ordinary quality at the latter

${ }^{1}$ Decker, Wis. experiment station, report 16, p. 155. 
temperature. While the temperature has therefore practically no importance, the air incorporated in the milk during the milking process will introduce an appreciable error in the testing, and samples of milk should therefore be left for an hour or more after milking before the milk is measured into the test kottles. By this time the specific gravity of the samples can also be correctly determined (113).

I7I. Size of the testing sample. Four ounces are a sufficient quantity for a sample of milk if it is desired to determine its per cent. of fat only; if the milk is to be tested with a lactometer, when adulteration is suspected, about a pint sample is needed. If this sample of milk is put into a bottle and carried or sent away from the farm to be tested, the bottle should be filled with milk clear up to the neck to prevent a partial churning of butter in the sample during transportation (30).

172. Variations in herd milk. While considerable variations in the quality of the milk of single cows are often met with, a mixture of the milk of several cows, or of a whole herd, is comparatively uniform from day to day; the individual differences tend to balance each other so that variations, when they do occur, are less marked than in case of milk of single cows. There are, however, at times marked variations also in the test of herd milk on successive days; the following figures from the dairy tests conducted at the World's Columbian Exposition in Chicago in 1893 illustrate the correctness of this statement. The tests included twenty-five Jersey and Guernsey cows each and twenty-four Shorthorn cows. 
Tests of herd milk on successive days.

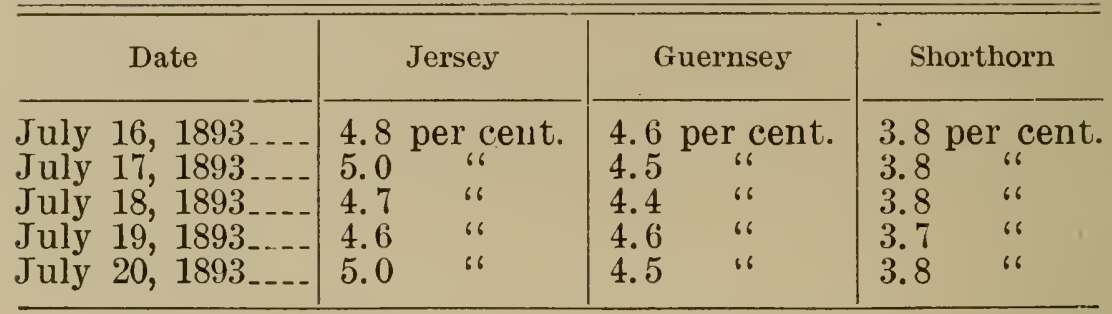

On July 17, 1893, the mixed milk of the Jersey cows tested two-tenths of one per cent. higher than on the preceding day; the Guernsey herd milk tested one-tenth of one per cent. lower, while the Shorthorn milk did not change in composition; comparing the tests on July 19 and 20, we find that the Jersey and Shorthorn milk tested four-tenths and one-tenth of one per cent. higher, respectively, on the latter day than on the former, and the Guernsey milk tested one-tenth of one per cent. lower. There was no change in the method of handling the cows. their feed, etc., on these days.

173. Ranges in variations of herd milk. According to Fleischmann, ${ }^{1}$ the composition of herd milk will vary on single days from the average values for the year, expressed in per cent. of the latter, as follows:

The specific gravity (expressed in degrees) may go above or below the yearly average by more than 10 per cent.

The per cent. of fat may go above or below the yearly average by more than 30 per cent.

The per cent. of total solids may go above or below the yearly average by more than 14 per cent.

The per cent. of solids not fat may go above or below the yearly average by more than 10 per cent.

To illustrate, if the average test of a herd during a whole period of lactation is 4.0 per cent., the test on a single day may

${ }^{1}$ Book of the Dairy, p. 32. 
exceed $4.0+\frac{30}{100} \times 4.0=5.2$, or may go below 2.8 per cent. (viz., $4.0-30-14.0$ ); if the average specific gravity is 1.031 (lactometer degrees, 31) the specific gravity of the milk on a single day may vary between 1.0279 and $1.0341 \quad\left(31+\frac{10}{10} \times 31=34.1\right.$; $31-100 \times 31=27.9$ ).

\section{I74. Influence of heavy grain-feeding on the qual-} ity of milk. If cows are not half-starved or underfed, an increase in the feeding ration will not materially change the richness of the milk produced; this has been shown by numerous careful feeding experiments conducted under a great variety of conditions and in many countries. Good dairy cows will almost invariably give more milk when their rations are increased, so long as they are not overfed, but the milk will remain of about the same quality after the first few days are passed as before this time, provided the cows are in good health and uuder normal conditions. Any change in the feed of cows will usually bring about an immediate change in the fat content of the milk, as a rule increasing it to some extent, but in the course of a few days, when the cows have become accustomed to their new feed, the fat content will again return to its normal amount.

I75. The records of the cows included in the feeding experiment at the Illinois station, to which reference has been made on p. 144, furnish illustrations as to the effect of heavy feeding on the quality of milk. The feed, as well as the milk of the cows, was weighed each day of the experiment. During the month of December each cow was fed a daily ration consisting of $10 \mathrm{lbs}$. of timothy hay, 20 lbs. of corn silage and 2 lbs. of oil meal; the table on p. 145 shows that cow No. 3 produced on

I See page 103. 
this feed an average of 12.1 lbs. of milk, testing 3.8 per cent. of fat. In January the grain feed was gradually increased until the ration consisted of $12 \mathrm{lbs}$. of timothy hay, 8 lbs. of corn and cob meal, 4 lbs. of wheat bran, and 4 lbs. of oil meal. All the cows gained in milk on this feed; cow No. 3 thus gave an average of 4 lbs. more milk per day in January than in December, but the average test of her milk was 3.7 per cent., or one-tenth of one per cent. lower than during the preceding month. The heavy grain-feeding was continued through February and March, when it reached 12 lbs. of timothy hay, 12 llss. of corn and cob meal, 6 lbs. of wheat bran and 6 lhs. of oil meal per day. The records show that the flow of milk kept up to 16 lbs. per day in February in case of this cow, but fell to 14 lbs. in March and April, the average test of the milk being, in February 3.6, in March 3.8, and in April 4.0 per cent. The milk was, therefore, somewhat richer in April than in December, but not more so than is found normally, owing to the progress of the period of lactation.

r76. Influence of pasture on the quality of milk. On May 1, the cows were given luxuriant pasture feed and no grain; a slight increase in the average amount of milk produced per day followed, with a reduction in the test, this being 3.8 per cent., the same as in December.

During all these changes of feed there was, therefore, not much change in the richness of the milk, while the flow of milk was increased by the heavy grain feeding for several months, as well as by the change from grain. 
feeding in the barn to pasture feed with no grain. ${ }^{1}$ As a general rule, the test of the milk will be increased by a few tenths of a per cent. during the first couple of weeks after the cows have been turned out to pasture in the spring. The increase is perhaps due as much to the stimulating influence of out-door life after the confinement in the stable during the winter and spring, as to the change in the feed of the cows. After a brief period the milk will again change back to its normal fat content.

I77. The increase which has often been observed in the amount of butter produced by a cow, as a result of a change in feed, doubtless as a rule comes from the fact that more, but not richer milk is produced. The quality of milk which a cow produces is as natural to her as is the color of her hair and is not materially changed by any special system of normal feeding. ${ }^{2}$

${ }^{1}$ For further data on this point, see Cornell (N. Y.) exp. sta., bulle tins $13,22,36$ and 49 ; N. D. exp. sta., bull. 16 ; Kansas exp. sta., re port, 1Ss8; Hoard's Dairyman, 1896, pp. $924-5$; W. Va. exp. sta., b. 109.

${ }^{2}$ On this point numerous discussions hare taken place in the past in the agricultural press of this and foreign countries, and the subject has been under debate at nearly every gathering of farmers where feed. ing problems have beenconsidered. Many farmers are firm in their be lief that butter fat can be "fed into" the milk of a cow, and would take exception to the conclusion drawn in the preceding. The results of careful investigations by our best dairy authorities point conclusively, however, in the direction stated, and the evidence on this point is overwhelmingly against the opinion that the fat content of the milk can be materially and for any length of time increased by changes in the system of feeding. The most conclusive evidence in this line is perhaps the Danish co-operative cow-feeding experiments, conducted during the nineties with over 2,000 cows in all. The conclusion arrived at by the Copenhagen experiment station, under whose direction the experiments have been conducted, is: that the changes of fecd made in the different lots of cows included on the experiments bad practically no influence on the chemical composition (the fat content) of the milk produced. In 
r78. Method of improving the quality of milk. The quality of the milk produced by a herd can generally be improved by selection and breeding, i. e., by disposing of the cows giving poor milk, say below 3 per cent. of fat, and by breeding to a pure-bred bull of a strain that is known to produce rich milk. This method cannot work wonders in a day, or even in a year, but it is the only certain way we have of improving the quality of the milk produced by our cows.

It may be well in this connection to call attention to the fact that the quality of the milk which a cow produces is only one side of the question; the quantity is another, and an equally important one. Much less dissatisfaction and complaint about low tests among patrons of creameries and cheese factories would arise if this fact was more generally kept in mind. A cow giving 3 per cent. milk should not be condemned because her milk does not test 5 per cent.; she may give twice as much milk per day as a 5 per cent cow, and will therefore produce considerably more butter fat. The point whether or not a cow is a persistent milker is also of primary importance; a production of $300 \mathrm{lbs}$. of butter fat during a whole period of lactation is a rather high dairy standard, but one reached by many herds, as the average for all mature cows in the herd. It should be remembered that a high production of but-

these experiments grain feeds were fed against roots, oil cake, wheat bran or shorts; grain and oil cake were furthermore fed against roots, and roots were given as an additional feed to the standard rations tried,-in all cases with practically negative results so far as changes in the fat contents of the milk produced are concerned. 
ter fat in the course of the whole period of lactation is of more importance than a very high test.

\section{Questions.}

1. How does the test of the milk yielded by a cow generally change with the advance of the period of lactation?

2. Mention at least six causes of variations in the test of a cow's milk.

3. How is an accurate sample taken of a cow's milk?

4. Between which limits is the test of milk of single cows and of a herd likely to vary?

5. Will it introduce any error in the test of a cow's milk to measure out the sample directly after milking? If so, how much?

6. How many times should the milk of a cow be weighed and tested to calculatel the total production of milk and butter fat by the cow during a whole period of lactation?

7. What is an official test of a cow?

8. How does the test, as a general rule, change during the first couple of weleks after the cows are let out on pasture in the the spring?

9. How do.changes in the feed of a cow influence the quan. tity and the quality of her milk? 
CHAPTER X.

\section{COMPOSITE SAMPLES OF MILK.}

179. Shortly after milk testing had been introduced to some extent in creameries and cheese factories, it was suggested by Patrick, then of the Iowa experiment station, ${ }^{1}$ that a great saving in labor, without affecting

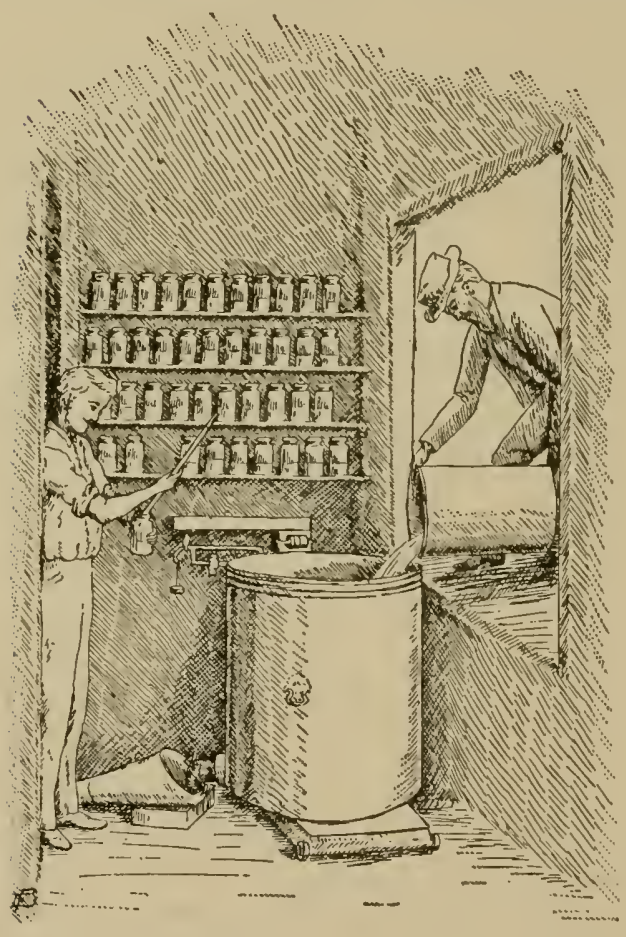

FIG. 51. Taking test samples at in-take. the accuracy of the results, could be obtained by testing a mixture of the daily samples of milk from one source, instead of each one of these samples. Such a mixture is called a composite sample. The usual methods of taking such samples at creameries and cheese factories are as follows :

I80. Methods of t a k in $g$ composite samples. a. Use of tin dipper. Either pint or. quart fruit jars, or milk bot. tles provided with a cover, are used for receiving the daily samples. One of these jars is supplied for each

${ }^{1}$ Bulletin 9, May 1890 . 
patron of the factory and is labeled with his name or number. A small quantity of preservative (bi-chromate of potash, corrosive sublimate, etc., see 190) is added to each jar; these are placed on shelves or somewhere within easy reach of the operator who inspects and weighs the milk as it is received at the factory. When all the milk delivered by a patron is poured into the weighing can and weighed, a small portion thereof, usually about an ounce, is put into the jar labeled with the name or number of the patron. The samples are conveniently taken by means of a small tin dipper holding about an ounce. This sampling is continued for a week, ten days, or sometimes two weeks, a portion of each patron's milk being added to his particular jar every time he delivers milk. A test of these composite samples takes the place of separate daily tests and gives accurate information regarding the average quality of the milk delivered by each patron during the period of sampling. The weight of butter fat which each patron brought to the factory in his milk during this time, is obtained by multiplying the total weight of milk delivered during the sampling period by the test of the composite sample, dividing the product by 100 .

18I. This method of taking composite samples has been proved to be practically correct. It is absolutely correct only when the same weight of milk is delivered daily by the patron. If this is not the case, the size of the various small samples should bear a definite relation to the milk delivered; one sixteen-hundredth, or one two-thousandth of the amount of milk furnished should, for instance, be taken for the composite sample from 
each lot of milk. This can easily be done by means of special sampling devices (see 182 et seq.). As the quantities of the milk delivered from day to day by each patron vary but little, perhaps not exceeding 10 per. cent. of the milk delivered, the error introduced by taking a uniform sample, e. g., an ounce of milk, each time is, however, small and it may not be necessary to take cognizance of it in factory work. This method of composite sampling described is quite generally adopted in separator creameries and cheese factories, where the payment for the milk is based on its quality.

In order to obtain reliable results by composite sampling it is essential that each lot of milk sampled shall be sweet and in good condition, containing no lumps of curdled milk or butter granules. The milk should of course always be evenly mixed before the sample is taken.

182. b. Drip sample. Composite samples are sometimes taken at creameries and cheese factories by collecting in a small dish the milk that drips through a fine hole in the bottom of the conductor spout through which the milk runs from the weighing ean to the receiving vat or tank. A small portion of the drip collected each day is placed in the composite sample jar, or the quantity of drip is regulated so that all of it may be taken. In the latter case the quantity of milk delivered will enter into the composite sampling as well as its quality and the sample from, say $200 \mathrm{lbs}$. of milk, will be twice as large as the sample from 100 lbs. of milk.

Where it is desired to vary the size of samples according to the quantity of milk delivered from day to day, 
it is necessary to adopt the method of collecting drip samples just explained, or to make use of special sampling devices, like the "milk thief," the Scovell, Equity, McKay, and Michels sampling tubes." The principle of these tubes is the same, and it will be sufficient to describe here only a few of them.

I83. c. The Scovell sampling tube. This convenient device for sampling milk ${ }^{2}$ (fig. 52) consists of a drawn copper or brass tube, onehalf to one inch in diameter; it is open at both ends, the lower end sliding snugly in a cap provided with three elliptical openings at the side, through which the milk is admitted. The milk to be sampled is poured into a cylindrical pail, or the factory weighing can, and the tube, with the cap set so that the apertures are left open, is lowered into the milk until it touches the bottom of the can. The tube will be filled instantly to the level of the milk in the can and is then pushed down against the bottom of the can, thereby closing the apertures of the cap and confining within the tube a column of milk representing exactly the quality of the milk sampling in the can and forming an aliquot part thereof.

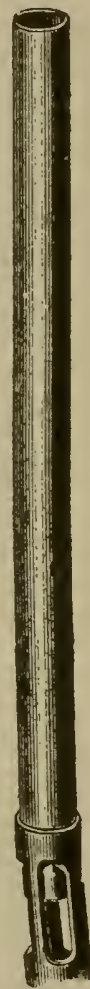
The milk in the sampling tube is then emptied into the composite sample jar by turning the tube upside down.

${ }^{1}$ A recent Wisconsin law (Chap. 99, laws of 1907) provides that in sampling cream or milk from which composite tests are to be made to determine the per cent of butter fat therein, no such sampling shall be lawful, unless a sample be taken from each weighing, and the quantity thus used shall be proportioned to the total weight of cream or milk tested.

${ }^{2}$ Kentucky experiment station, Sth report, pp. xxvi-xxvii. 
184. If the diameter of the sampling pail used is 8 inches, and that of the sampling tube $1 / 2$.inch, the quantity of milk secured in the tube will always stand in the ratio to that of the milk in the pail, of $(1 / 2)^{2}$ to $8^{2},{ }^{1}$ that is, as 1 to 256 , no matter how much or how little milk there is in the pail, the sample will represent $\frac{1}{2} \frac{1}{56}$ part of the milk. For composite sampling of the milk of single cows, this proportion will prove about right; if more milk is wanted for a sub-sample, dip twice, or pour the milk to be sampled into a can of smaller diameter. If the mixed milk from a number of cows is to be sampled, a wider sampling can may be used. By adjusting the diameter of the tube or the can, any desired proportion of milk can be obtained in the sample.

For factory sampling, with a weighing can 26 inches in diameter, a tube three-quarters of an inch in diameter will be found of proper dimensions.

In using these tubes, the milk or cream must in all cases be in cylindrical cans when the sample is drawn.

The sampling tube will furnish a correct sample of the milk in the can, even if this has been left standing for some time; it is better, however, to take out the sample soon after the milk has been poured into the can, as the possible error of cream adhering to the sides of the sampling tube is then avoided.

185. The accuracy of the sampling of milk by means of the Scovell tube was proved beyond dispute in the breed tests conducted at the World's Columbian Expo-

1 The contents of a cylinder are represented by the formula $9 \mathrm{r}^{2} \mathrm{~h}, r$ being the radius of the cylinder, and $h$ its height. The relation between two cylinders of the same height, the radii of which are $R$ and $r$, is therefore as $\pi R^{2} h$ to $\pi r^{2} h$, or as $R^{2}$ to $r^{2}$. 
sition in 1893, in which tests this method was adopted for sampling the milk produced by the single cows and the different herds. ${ }^{1}$ The data obtained in these breed tests also furnish abundant proof of the accuracy of the Babcock test.

r86. d. The McKay sampler (fig. 53), constructed by Professor G. L. McKay, formerly of Iowa experiment

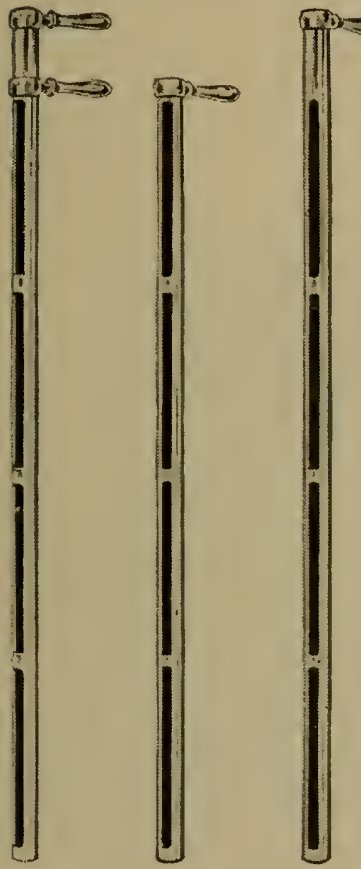

Fig. 53. The McKay sampler. station, consists of two nickel-plated $\rightarrow$ brass tubes that telescope one within the other; both have a milled slot so made that when the handles stand together the slot is open; by turning the handles at right angles the slot is closed. The sampler is made in two lengths, 21 and 18 inches, and has been found very convenient for sampling either milk or cream.

I86a. e. Michels' cream-sampling tube consists of a modified Scovell sampler in a tin jacket. It was invented by Professor John Michels, late of North Carolina agricultural college. This sampler renders possible an accurate and rapid sampling of any cream, regardless of its richness and acidity, without stirring the cream.

\footnotetext{
${ }^{1}$ Kentucky experiment station, 8th report, pp. xxx-xxxi. Another form of a milk sampling tube in use at the Iowa experiment station was described and illustrated by Mr. Eckles in Breeder's Gazette, May $19,1897$.
} 
187. f. Composite sampling with a "one-third sample pipette." Milk is sometimes sampled directly from the weighing can into the Babcock test bottle by means of a pipette holding 5.87 cc., which is one-third the size of the regular pipette. This quantity is measured into the test bottle from three successive lots of milk from the same patron and the test then made in the ordinary manner. In this way one test shows the average composition of the milk delivered during three successive days or deliveries. When this method is adopted, as many test bottles are provided as there are patrons; there is no need of using any preservatives for milk in this case. Fig. 55 shows a convenient rack for holding the test bottles used in composite sampling with a "one-third sample pipette."

Accurate results can be obtained by this method of sampling, if care is taken in measuring out the milk, and if it is not frozen or contains lumps of cream. It is doubtful if the method has any advantage over the usual method of composite sampling. If milk is delivered daily and each lot is sampled with the one-third pipette, twice or three times the number of tests are required as when composite samples are taken in jars and tested once every week or ten days. This method furthermore takes a little more time in the daily sampling than the other, as the quantity of milk must be

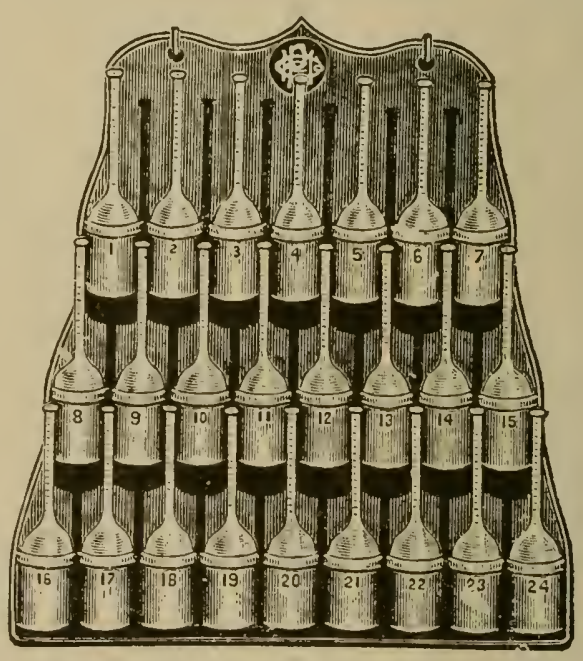

FIG. 55. Test-bottle rack for use in creameries and cheese factories. measured out accurately each time. If a test bottle is accidently broken or some milk spilled, the opportunity of ascertaining the fat content of the milk delivered during the three days is lost; if a similar accident should occur in testing composite samples collected in jars, another test can readily be made. 
188. Accuracy of the described methods of sampling. An experiment made at the Wisconsin Dairy School may here be cited, showing that concordant results will be obtained by the use of the drip sampling method and the Scovell tube. Two composite samples were taken from fifty different lots of milk, amounting to about 6,000 lbs. in the aggregate. One sample was taken of the drip from a hole in the conductor spout through which the milk passed from the weighing can; the other was taken from the weighing can by means of a Scovell sampling tube. The following percentages of fat were found in each of these samples: ${ }^{1}$

\begin{tabular}{l|c|c}
\hline \hline & Babcock test & $\begin{array}{c}\text { Gravimetric } \\
\text { analysis }\end{array}$ \\
${$\cline { 2 - 3 }$} }$ & $\begin{array}{l}4.0 \text { per cent. } \\
\text { Scovell tube composite sample }\end{array}$ & $\begin{array}{l}4.04 \text { per cent. } \\
4.06 \text { per cent. }\end{array}$ \\
\hline
\end{tabular}

Preservatives for Composite Samples.

189. When milk is kept for any length of time under ordinary conditions, it will soon turn sour and become loppered, and further decomposition shortly sets in, which renders the sampling of the milk both difficult and unsatisfactory (19). The period during which milk will remain in an apparently sweet or fresh condition varies with the temperature at which it is kept, and with the cleanliness of the milk. It will not generally remain sweet longer than two days at the outside, at ordinary summer or room temperature.

In order to preserve composite samples of milk in a proper condition for testing, some chemical which will check or prevent the fermentation of the milk must be

${ }^{1}$ See also 199 et seq. 
added to it. A number of substances have been proposed for this purpose.

190. Bi-chromate of potash. This preservative is preferred by many because it is relatively harmless, cheap and efficient. The bi-chromate method for preserving samples of milk was proposed by Mr. J. A. Alen, city chemist of Gothenburg, Sweden, in 1892, and has been generally adopted in dairy regions in this country and abroad. While not perfectly harmless, the bi-chromate is not a violent poison like other chemicals proposed for this purpose, and no accidents are liable to result from its use.

I9r. The quantity of bi-chromate necessary, for preserving half a pint to a pint of milk for a period of one or two weeks is about one-half gram (nearly 8 grains).

According to Winton and Ogden, ${ }^{2}$ a .22-inch pistol cartridge shell $1 / 2$ inch long, or a .32-inch ealiber shell $1 / 4$ inch long, when loosely filled, will hold enough powdered bi-chromate to preserve $1 / 2$ pint, and a .32-inch caliber shell $1 / 2$ inch long will hold enough to preserve one pint. These shells may be conveniently handled by soldering to them a piece of stiff wire which serves as a handle. The amount of bi-chromate placed in each composite sample jar would fill about half the space representing one per cent. in the neck of a Babcock milk test bottle.

I92. The first portions of milk added to the composite sample jars containing the specified amount of

${ }^{1}$ Biedermann's Centralblatt, 1892, p. 549.

${ }^{2}$ Connecticut experiment station, report for 1884 , p. 222. 
bi-chromate will be colored almost red, but as more milk is added day by day, its color will become lighter yellow. The complete sample should have a light straw color; such samples are most easily mixed with acid when tested. If more bi-chromate is used, the solution of the casein in the acid is rendered difficult and requires persistent shaking. Bi-chromate can be bought at drug stores or from dairy supply dealers at about 30 cents a pound. Powdered bi-chromate of potash should be ordered, and not crystals, as the latter dissolve only slowly in the milk. Bi-chromate tablets containing the correct quantity of preservative for a quart or pint sample have lately been placed on the market and will be found convenient.

\section{Corrosive sublimate tablets for composite} samples. During late years corrosive sublimate tablets have come into general use in factories. These contain mercuric chlorid with anilin color (rosanilin). ${ }^{1}$ The coloring matter is added to give a rose color to the sample preserved, thus showing that the milk is not fit for consumption; the bi-chromate giving naturally a yellow color to the milk, renders unnecessary the addition of any special coloring matter.

Compounds containing corrosive sublimate are violent poisons and must always be handled with the greatest care, lest they get into the hands of children or persons not familiar with their poisonous properties; they will preserve the milk longer than bi-chromate when applied in sufficient quantities.

${ }^{1}$ Iowa experiment station, bulletins 9, 11, 32. 
Among other substances recommended for use in butter or cheese factories as milk preservatives for composite samples may be menticned formaldehyde, boracicacid compounds, chloroform, carbon bi-sulfid., ${ }^{1}$ copper ammonium sulfate, sodium fluorid and ammonia glycerin (sp. gr., 1.031).

194. Care of composite samples. The composite sample jars should be kept covered to prevent loss by evaporation, and in a cool, dark place, or at least out of direct sunlight when bi-chromate of potash is used as a preservative; the chromic acid formed by the reducing influence of light on chromate solutions produces a leathery cream which it is difficult to dissolve in sulfuric acid.

A coating of white shellac has been suggested to protect the labels of the composite sample jars. The shellac is applied after the names of the patrons have been written on the labels, and when these have been put on the jars. Gummed labeis, $1 \times 21 / 2$ inches, answer this purpose well.

Numbers are sometimes ground on the sample jar or stamped on brass tags attached to the jars by a wire.

In keeping the milk from day to day, care should be taken that the cream forming $\mathrm{cn}$ the milk does not stick to the sides of the jars in patches above the level of the milk. Unless the daily handling of the jars and the addition of fresh portions of milk be done carefully, the cream will become lumpy and will dry on the sides of the jars. In some cases it is nearly impossible to

1 Delaware experiment station, eighth report, 1896 , which also see for trials with a large number of different preservatives. 
evenly distribute this dried cream through the entire sample at testing time so as to make the composite sample a true representative of the different lots of milk from which it has been taken.

195. Every time a new portion of milk is added to the jar this should be given a gentle horizontal rotary motion, thereby mixing the cream already formed in the jar with the milk and loosening the cream sticking to its side. This manipulation also prevents the surface of the milk from becoming covered with a layer of partially dried leathery cream.

Composite samples having patches of dried cream on the inside of the jar are the result of carelessness or ignorance on the part of the operator. If proper attention is given to the daily. handling of the composite samples, the cream formed in the jars can again be evenly mixed with the milk without difficulty.

196. Fallacy of averaging percentages. A composite sample of milk should represent the average quality of the various lots of milk of which it is made up. This will be true if a definite aliquot portion or fraction of the different lots of milk is taken. If the weights of, say ten different lots of milk, are added together and the sum divided by ten, the quotient will represent the average weight per lot of mill, but an average of the tests of the different lots obtained in this way may not be the correct average test of the entire quantity of milk. The accuracy of such an average figure will depend on the uniformity in the composition and weights of the ten lots of milk. When there is no uniformity, the weights of the different lots of milk as well as their 
tests must be considered. The following example will illustrate the difference between the arithmetical average of a number of single tests and the true average test of the various lots.

\section{Methods of calculating average percentages.}

\begin{tabular}{|c|c|c|c|c|c|c|c|}
\hline \multicolumn{4}{|c|}{ I. Milk varying in weights and tests. } & \multicolumn{4}{|c|}{ II. Milk of uniform weights and tests. } \\
\hline Lot & 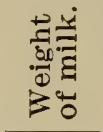 & 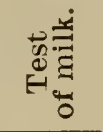 & 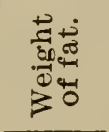 & LOT & 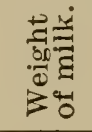 & 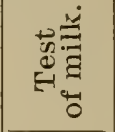 & 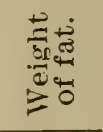 \\
\hline $\begin{array}{l}\text { II } \\
\text { II } \\
\text { III } \\
\text { II } \\
\text { V }\end{array}$ & $\begin{array}{r}\text { lbs. } \\
120 \\
570 \\
360 \\
55 \\
82 \\
\end{array}$ & $\begin{array}{c}\text { per ct. } \\
3.5 \\
5.0 \\
5.2 \\
3.0 \\
4.0 \\
\end{array}$ & $\begin{array}{r}1 \mathrm{bs} . \\
4.2 \\
28.5 \\
18.7 \\
1.6 \\
3.2 \\
\end{array}$ & $\begin{array}{l}\text { II } \\
\text { II } \\
\text { III } \\
\text { IV } \\
\text { IV } \\
\text { V }\end{array}$ & $\begin{array}{r}\text { lbs. } \\
250 \\
225 \\
240 \\
238 \\
234 \\
\end{array}$ & $\begin{array}{c}\text { per ct. } \\
4.2 \\
4.0 \\
4.3 \\
4.1 \\
4.4 \\
\end{array}$ & $\begin{array}{r}\text { lbs. } \\
10.5 \\
9.0 \\
10.3 \\
9.7 \\
10.3 \\
\end{array}$ \\
\hline Total & 1187 & .... & 56.2 & Total $\ldots . . .$. & 1187 & -........ & 49.8 \\
\hline Average .. & 237 & 4.14 & 11.24 & Average .... & 237 & 4.20 & 10.0 \\
\hline $\begin{array}{l}\text { True aver- } \\
\text { age test ... }\end{array}$ & (-........ & $4.73^{*}$ & (...... & $\begin{array}{c}\text { True average } \\
\text { test }\end{array}$ & & $4.22 \dagger$ & \\
\hline & $\frac{2 \times 100}{1187}=$ & & & & $\frac{9.8 \times 100}{1187}$ & 1.22 & \\
\hline
\end{tabular}

197. The figures given in the table show that when the different lots of milk vary in test and weight, as in the first case, the correct average test of the $1187 \mathrm{lbs}$. of milk is not found by dividing the sum of these tests by five, which would give 4.14 per cent.; but the percentage which 56.2 (the total amount of fat in the mixed milk) is of 1187 (the total amount of milk), is 4.73 , and this is the correct average test of the mixed milk made up of the five different lots.

In the second case, the variations in both the weights of the different lots of milk and their tests, are comparatively small, and both methods of calculation give 
therefore practically the same average test; but also in this case, the correct average test is found by dividing the total amount of fat by the total quantity of milk, making 4.22 per cent., instead of 4.20 per cent., which is the arithmetical mean of the five tests. The quantities of milk in the various lots do not enter into the calculation of the latter. ${ }^{1}$

198. The second example represents more nearly than the first one the actual conditions met with at creameries and cheese factories. As a rule, the mixed milk from a herd of cows does not vary more in total weight or tests, within a short period of time like one to two weeks, than the figures given in this example. On account of this fact, samples taken, for instance, with a small dipper may give satisfactory results to all parties concerned. If the different lots of milk varied in weight and test from day to day, as shown in the first case, it would be necessary to use a "milk thief" or one of the sampling tubes for taking the composite samples; the size of each of the samples taken would then represent an exact aliquot portion of the various lots of milk (182).

199. A patron's dilemma. The following incident will further explain the difficulties met with in calculating average tests of different lots of milk.

The weekly composite sample of the milk supplied by a creamery patron from his herd of 21 cows tested 4.0 per cent. fat. One day the farmer brought to the creamery a sample of the morning's milk from each of his cows, and had them tested; after adding the tests together and dividing the sum by 21 , he

\footnotetext{
${ }^{1}$ In the experiment given on p. 148, the arithmetical mean of the tests is 5.15 per cent., while the true average fat content of mills is 4.85 per cent.
} 
obtained an average figure of 5.1 per cent. of fat. From this he concluded that the average test of the milk from his cows ought to be 5.1, instead of 4.0, and naturally asked for an explanation.

The first thing done was to show him that while 5.1 was the sorrect average of the figures representing the tests of his twenty-one cows, it was not a correct average test of the mixed milk from all his cows, as he had not considered, in calculating this average, the quantities of milk yielded by each cow; the following illustration was used:

Cow No. 1, yield 25 lbs. of milk, test 3.6 per cent. $=0.9 \mathrm{lb}$. of butter fat.

Oow No. 2, yield $6 \mathrm{lbs}$. of milk, test 5.0 per cent. $=0.3 \mathrm{lb}$. of butter fat.

$$
\text { Total.....31 lbs. } \quad \frac{2) 8.6}{4.3} \text { per cent. }
$$

The two cows gave $31 \mathrm{lbs}$. of milk containing $1.2 \mathrm{lbs}$. of fat; the test of the mixed milk would therefore not be 4.3 per cent. $\left(\frac{3.6+5.0}{2}\right)$, but $\frac{1.2 \times 100}{31}=3.87$ per cent. If the fat in the mixed milk was calculated by the average figure 4.3 per cent., 1.33 lbs. of fat would be obtained, i. e., $0.13 \mathrm{lb}$. more than the cows produced.

In order to further demonstrate the actual composition of the mixed milk of the twenty-one cows, the milk of each cow was weighed and tested at each of the two milkings of one day. The weights and tests showed that the cows produced the following total number of pounds of milk and of fat:

Morning milking, 113.3 lbs. of milk, containing $5.17 \mathrm{lbs}$. of fat. Night milking, $130.9 \mathrm{lbs}$. of milk, containing $4.98 \mathrm{lbs}$. of fat. The morning milk therefore contained $\frac{5.17 \times 100}{113.3}=4.56$ per cent. of fat, and the night milk, $\frac{4.98 \times 100}{130.9}=3.80$ per cent. of fat.

The sum of the morning and night milkings gave: milk, -244.2 lbs., fat $10.15 \mathrm{lbs}$. The mixed morning and night milk, therefore, contained $\frac{10.15 \times 100}{244.2}=4.1$ per cent. of fat. This is the true average test of the morning and night milkings of these twentyone cows, as found by weighing and testing separately the milk of each cow at both milkings.

The total milk was strained into a large can at the farm, both in the morning and in the evening. A sample of the mixed milk 
was in each case taken with a long-handled dipper as soon as the milkings were finished. When the cans of milk were delivered at the creamery, a sample of each was taken with a Scovell sampling tube. The tests of these four samples are given below, together with the results of the individual tests:

\begin{tabular}{|c|c|c|}
\hline & Morning Milk. & Night Milk. \\
\hline Sample taken at the farm, with dip- & \multirow{4}{*}{$\begin{array}{l}4.4 \text { per ct. } \\
4.5 \text { 66 6 } \\
4.5 \text { 6 } 6\end{array}$} & $38 n$ \\
\hline Sample taken at creamery with Sco- & & 3.8 per ct. \\
\hline Calculated from weights and tests of & & 3. \\
\hline milk from each cow & & 3.8 " 6 \\
\hline
\end{tabular}

The figures given show that practically uniform tests were obtained by the different methods of sampling.

\section{Questions.}

1. What is a composite sample of milk:

2. Describe the proper care of composite samples.

3. Give an example showing that composite samples of milk may be inaccurate when taken with a small dipper.

4. Describe the construction of the following methods of sampling milk or cream, by (a) drip sample, (b) the Scovell, (c) the McKay, and (d) the Michels' sampling tubes.

5. What is the purpose of adding preservatives to milk or cream samples? Mention the more common preservatives used and quantities to be added. 


\section{CHAPTER XI. \\ CREAM TESTING AT CREAMERIES.}

200. The cream delivered at gathered-cream factories is now tested by the Babcock test in many localities, and this has been adopted as a basis of paying for the cream in the same manner as milk is paid for at separator creameries. It has been found to be more satisfactory to both cream buyer and seller than either the oil-test churn or the space (or gauge) systems which were previously used for this purpose.

The details of the application of the Babcock test to the practical work at cream-gathering creameries have been carefully investigated by Winton and Ogden in Connecticut, ${ }^{1}$ Bartlett in Maine, ${ }^{2}$ and Lindsey in Massachusetts, ${ }^{3}$ and we also owe to the labors of these chemists much information concerning the present workings of other systems of paying for the cream delivered at creameries.

201. The space system. Numerous tests have shown that one space or gauge of cream does not contain a definite, uniform amount of fat. In over 100 comparisons made by Winton it was found that one space of cream $^{4}$ contained from .072 to $.170 \mathrm{lb}$. of butter fat, or

1 Conn. experiment station (New Haven), bull. 108 and 119 ; report 1894, pp. 214-244.

${ }^{2}$ Maine experiment station, bull. 3 and 4 (S. S.)

${ }^{3}$ Hatch experiment station, report 1894 , pp. $92-103$; 1895, pp. 67-70.

4 The space is the volume of a cylinder, $81 / 2$ inches in diameter and $6 \frac{19}{4}$ of an inch high. The number of spaces in each can of milk is read off before skimming by means of a scale marked on a strip of glass in the side of the can (Conn. exp. sta., bull. 119). 
on the average $.13 \mathrm{lb}$., and the number of spaces required to make one pound of butter varied from 5.01 to 11.72. It is also claimed that in the winter season when the cream is gathered at long intervals, like once a week, it is necessary for the buyer to accept the seller's statement of the record of the number of cream spaces which he furnishes, since the cream cannot be left in the creaming can for so long a time. These objections to the space system apply only to the method of paying for the cream, and not to the manner in which the cream is obtained.

202. The oil-test churn. As stated in the introduction, the oil-test churn (fig. 56) has been used quite extensively among gathered-cream factories; this system is based on the number of inches of cream which the various patrons deliver to the factory; a creamery inch is the quantity of cream which will fill a can twelve inches wide, one inch high; it contains 113 cubic inches. ${ }^{1}$ This

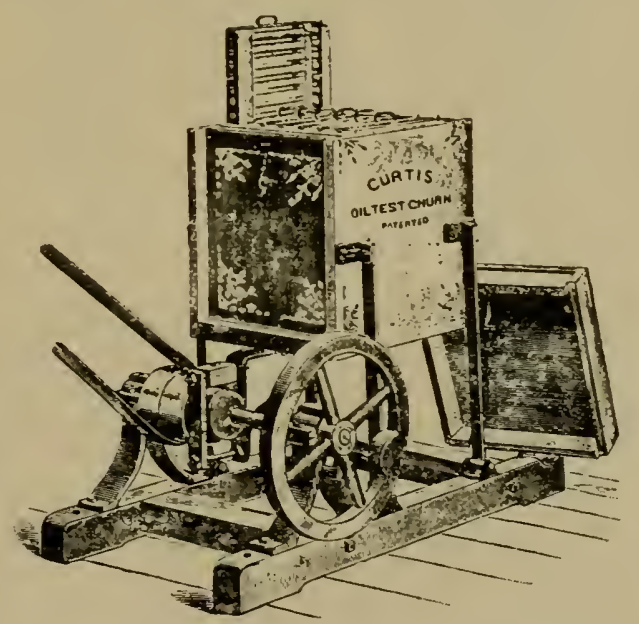

FIG. 56. The oil-test churu. quantity was supposed to make one pound of butter. In using this method the driver pours the patron's cream into his 12-inch gathering pail, measures it with

1 A layer of two inches in an 8-inch pail contains 100.531 cubic inches, two inches in a $8 \%$-inch pail 110.18 cubic incues, rnd two inches in a $81 / 2$-inch pail 113.49 cubic inches.

12 
his rule and records the depth of the cream in the can, in inches and tenths of an inch. The cream is then stirred thoroughly with a ladle or a stout dipper, and sampled by filling a test tube to the graduation mark by means of a small conical dipper provided with a lip. A driver's case contains either two or three "cards," holding fifteen test tubes each (see fig. 57).

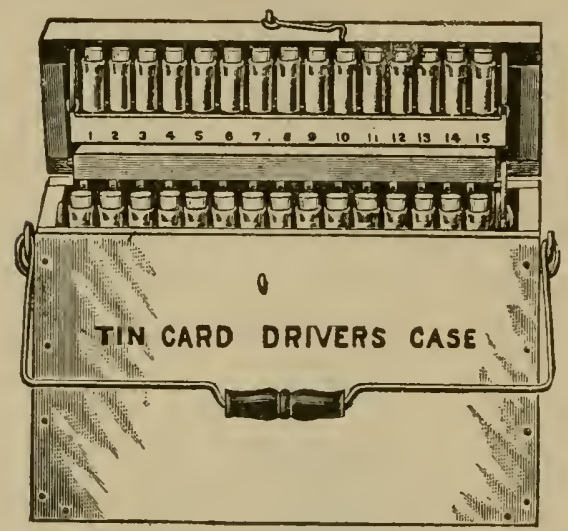

FIG. 57. Cream-gatherer's sample case.

The tubes as filled are placed in the case and the corresponding number in each instance recorded in front of the patron's name, together with the number of inches of cream fur. nished by him.

On the arrival at the creamery the tin cards holding the tubes are placed in a vessel filled with water of the churning temperature (say, $60^{\circ}$ in summer and $65^{\circ}$ to $70^{\circ}$ in winter). When ready for churning they are placed.in the oil-test churn (fig. 56), the cover of the churn put on, and the samples of cream churned to butter. On the completion of the churning, the cards are transferred to water of $175-190^{\circ} \mathrm{Fahr}$., where they are left for at least ten minutes to melt the butter and "cook the butter milk into a curd." The oil will now be seen mixing through the mass. The test tubes are then cooled to churning temperature and churned again, by which process the curd is broken into fine 
particles, which, when the butter is re-melted, will settle to the bottom. The butter is melted after the second churning by placing the tubes in water at $150-175^{\circ}$ F., allowing them to remain therein for at least twenty minutes. Some samples must be churned three or four times before a good separation of oil is obtained. A clear separation of oil is often facilitated by adding a little sulfuric acid to the tubes.

The length of the column of liquid butter fat is determined by means of a special rule for measuring the butter oil; this rule shows the number of pounds and tenths of a pound of butter which an inch of cream will make; the first tenth of a pound on the rule is divided into five equal parts, so that measurements may be made to two-hundredths of a pound. The melted fat is measured with the rule, by raising the tin card holding the bottles to about the height of the eye; the reading is recorded on the driver's tablet under Test per inch, opposite the number of the particular patron. The test multiplied by the inches and tenths of an inch of cream supplied will give the amount of butter in pounds, with which the patron will be credited on the books of the creamery.

203. The objection to this system of ascertaining the quality of cream delivered by different patrons lies in the fact that it determines the churnable fat, and not the total fat of the cream; the amount of the former obtained depends on many conditions beyond the control of the patron, viz., the consistency, acidity and temperature of the cream, the size of the churn or churn- 
ing vessel, etc. ${ }^{1}$ The same reasons which caused the churn to be replaced by methods of determining the total fat of the milk, in the testing of cows among dairymen and breeders, have gradually brought about the abandonment of the oil test in creameries and the adoption of the Babcock test in its place. It may be said, on the other hand, in favor of the use of the oil test in creameries that it is a considerably cheaper method than any fat test, and takes less labor and time on the part of the operators than do the latter methods.

204. The Babcock test for cream. Both the space system and the oil-test churn used for estimating the quality of cream at creameries have now largely been replaced by the Babcock test in the more progressive creameries in this country, and composite samples of cream are collected and tested in a similar manner as is done with milk at separator creameries and cheese factories.

A very satisfactory method of arrangements for working the Babcock test, in use in many eastern creameries, is described by Winton and Ogden in the Connecticut report previously referred to. The cream gatherer who collects the cream in large cream cans is supplied with a spring balance (1, see fig. 58 ), a pail for sampling and weighing the cream (2), sampling tube (3), and collecting bottles (5). At each patron's farm he takes from his wagon the sampling pail and tube, the scales. and one small collecting bottle. He should

1 It follows from this that there can be no definite relation between the results obtained by the Babcock test and the oil-test readings; a reading of 100 in the oil-test is, however, on the average, equivalent to about 23 per cent. of butter fat in the cream. 
find in the dairy of the patron the cans of perfectly sweet cream, kept at a temperature of $40^{\circ}$ to $50^{\circ} \mathrm{F}$.,

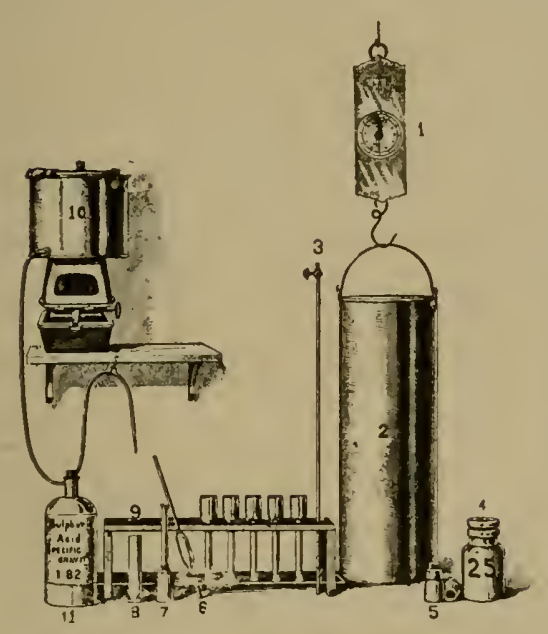

FIG. 58. Outfit for cream testing by the Babcock test at gatheredcream factories.

and protected from dirt and bad odors. Either sour or frozen cream must be rejected. The patron's number should be painted in some conspicuous place near the cream cans in his dairy house. The gatherer hangs the scale on a hook near the cream to be collected; the scale should be made so that the hand of the dial will stand at zero when the empty pail is hung on it. The cream is then poured at least twice from one can to another in order to mix it thoroughly. ${ }^{1}$ 205. When properly mixed, the cream is poured into the weighing pail and is weighed and sampled. The authors give the following description of the cream sampling tube used, and directions for sampling and weighing the cream.

"Sampling Tube.-This tube is of stout brass, about $\frac{1}{32}$ of an inch thick, and a few inches longer than the weighing pail which

1 The necessity of care in mixing the cream is shown by the following illustration given by the authors referred to.

Per cent of fat in cream which stood for 24 hours.

Sample drawn

Surface.

Not mixed 28.00 23.75

Poured

once.
Bottom 5.00

22.00

Poured

twice.

19.25

22.50

22.25 
is used with it. On the upper end, a small brass stop-cock of the same bore is fastened. It should be nickel plated inside and out, to keep the metal smooth and free from corrosion. These tubes may be obtained from less than $\frac{3}{16}$ to over $1 / 4$ inch bore. The greater the diameter of the weighing pail, the wider should be the bore of the tube. For use with pails 8 inches in diameter, a $\frac{3}{1 \overline{6}}$ inch bore sampling tube will serve the purpose, but when the pail has a diameter of 9 or more inches, a tube with a bore of $1 / 4$ inch or more should be used. It must be borne in mindthat doubling the diameter of the pail, or of the sampling tube, increases its capacity fourfold.

"The tube when not in use should be kept in an upright position to permit draining.

"Sampling and Weighing.-Lower the sampling tube, cock and up, with the cock open, to the bottom of the weighing pail which holds the mixed cream. When it is filled raise it out of the liquid and allow it to drain for a few seconds. By this means the tube is rinsed with the cream to be sampled and any traces of cream adhering to the tube from previous use are removed. With the cock still open, slowly lower the sampling tube to the bottom of the cream pail. After allowing a moment for the cream to rise in the tube to the same height as in the pail, close the cock and raise the sampler carefully out of the cream. As long as the cock is closed, the cream in the tube will not flow out, unless the tube is strongly jarred. Allow the cream adhering to the outsidel of the tube to drain off for a few seconds, then put the lower end into the 1 to $1 \frac{1}{2} \mathrm{oz}$. wide-mouth glass collecting bottle which bears the patron's number on its cork, and open the cock. The cream will then flow out of the sampler into the bottle, which is afterwards securely corked and put into the cream gatherer's case. Immediately weigh the cream in the cream pail to the quarter or half pound, as may be judged expedient, and record the weight.

"If the patron has more than one pailful, repeat with each pailful the operation of sampling and weighing, putting all the samples in one and the same bottle. Weigh all cream collected in one and the same sampling pail and draw a sample from each separate portion weighed.", 
206. After sampling and weighing each patron's cream it is poured into the driver's large can, and the sample bottles are carried in a case to the creamery where the contents of each bottle is poured into the composite sample jar of the particular patron. The samples of cream in the small bottles, besides furnishing the means of testing the richness of the cream, give the creamery man an opportunity to inspect the flavor of each lot of cream, and the condition in which it has been kept by the various patrons. Some preservative, usually corrosive sublimate tablets, is placed in the composite sample jars, and these are cared for and tested in the same manner as composite samples of milk (194).

207. The collecting bottles should be cleaned with cold, and afterwards with hot water; as soon as they are emptied, and before a film of cream dries on them. When washed and dried, these bottles are placed in the cases, ready for the next collecting trip. There can be no confusion of bottles since the corks and not the bottles are marked with the numbers of the respective patrons.

208. When this system of testing composite samples is adopted, the patrons are paid for the number of pounds of butter fat contained in their cream, in the same way as milk is paid for at separator creameries. It makes no difference how thick or how thin the cream may be, or how much skim milk is left in the cream when brought to the factory. Eighty pounds of cream containing 15 per cent. of fat is worth no more nor less than 48 pounds of cream testing 25 per cent.; in either case 12 pounds of pure butter fat is 
delivered. This will make the same amount of butter in either case, viz., about 14 lbs., and both patrons should therefore receive the same amount of money.

There is a small difference in the value of the two lots of cream to the creamery owner or the butter maker, in favor of the richer cream, both because its smaller bulk makes the transportation and handling expenses lighter, and because slightly less butter fat will be lost in the butter milk, a smaller quantity of this being obtained from the richer cream. But it is doubtful if the differences thus occurring are of sufficient importance to be noticed under ordinary creamery conditions; the example selected presents an extreme case of variation in the fat content of cream. A trial of this system at five Connecticut creameries, supplied mostly with Cooley cream by over 175 patrons, showed that the average composition of the cream from the different patrons varied only from 16.9 to 19.8 per cent. of fat. The cream of some patrons on certain days contained only 9.5 per cent. of fat, and other patrons at times had as high a test as 30 per cent., but these great differences largely disappeared when the average quality of the cream delivered during a period of time, like a month or more, was considered.

209. Smaller differences in the composition of cream will, however, always occur, even if the same system of creaming the milk, like the centrifugal process, is used and all factors remain as nearly the same as possible at all times. This is due to differences in the composition of the milk and its creaming quality; whether largely from fresh cows or from late milkers; whether kept 
standing for a time before being set, or submerged in the creamer immediately after milking and straining, diameter of creaming cans, etc. Bartlett states ${ }^{1}$ that the percentage of fat in the cream from the same cows may be increased ten per cent. or more by keeping the water at $70^{\circ}$ instead of at $40^{\circ} \mathrm{F}$. The higher temperature will give the richer cream, but the separation will not be so complete, since a richer skim milk is obtained from the milk set at this temperature. Separator cream is not materially influenced by the conditions mentioned, as the separator can be regulated to deliver cream of nearly uniform richness from all kinds of sweet milk.

210. At creameries where both milk and cream are delivered, somewhat of an injustice is done to patrons delivering cream, by paying for the amounts of butter fat furmished by the different patrons. By multiplying the cream fat by $1.03,{ }^{2}$ the value of his products to the creamery is taken into proper account, and justice is done to all parties concerned ${ }^{3}$ (239).

\section{Gathering and sampling hand-separator} cream. On account of the great variation in both the richness and the purity of farm separator cream it has been found in practice that composite samples of cream are not so satisfactory to either buyer or seller as the testing of a sample taken from each lot of cream gathered. A still more satisfactory method is to provide separate cans for each patron, the cream gatherer leav-

${ }^{1}$ Bull. 3 (S. S.), Maine experiment station.

2 Spillman (Dairy and Creamery, Chicago, April 1, 1899) recommends the use of the factor 1.044 .

8 This subject is discussed in detail in the 17 th annual report of Wis. experiment station, pp. $90-92$; see also the 20 th report of that Station, pp. 130-31. 
ing an empty, clean can at each farm and taking a full or partially filled can of cream from the farm to the factory. This makes it necessary for the cream gatherer to carry as many cans as he has patrons to gather cream from, but it gives the factory operator a chance to inspect, weigh and sample the cream from each farm and relieves the cream gatherer of all these details which are often the cause of dissatisfaction.

\section{Questions.}

1. In what ways do the results obtained with the oil-test churn differ from those obtained with the Babcock test?

2. Describe the method of testing cream by the Babcock test at gathered-cream factories.

3. What advantages has the gathering of cream in separate cans over mixing the cream from all the patrons of one route? 
CHAPTER XII.

\section{CALCULATION OF BUTTER-AND CHEESE YIELD}

\section{A.-Calculation of Yield of Butter.}

212. Butter-fat test and yield of butter. The Babcock test shows the amount of pure butter fat contained in a sample of milk, cream or other dairy products. The butter obtained by churning crearn or milk contains, in addition to butter fat, a certain amount of water, salt and curd. While an accurate milk test gives the total quantity of butter fat found in the sample of milk or cream tested, the churn cannot be depended upon either to leave the same amount of butter fat in the butter milk or to include the same amount of water, salt or curd in the butter at each churning.

If a quantity of milk, say 3,000 lbs., be thoroughly mixed in a vat, and then divided into half a dozen equal portions, a Babcock test of the different lots will show the same percentage of butter fat in each portion. If, on the other hand, each of these lots be skimmed, and the cream ripened in different vats and churned separately, the same weight of butter from each lot of 500 lbs. of milk will not be obtained, even by the most expert butter maker, or if all the operations of skimming, cream ripening, churning, salting and butter-working were made as nearly uniform as possible. Careful operators can handle the milk and cream so that very nearly the 
same proportion of fat contained in the milk is recovered in the butter in different churnings, but since the water and salt in butter are held mechanically and are not chemically combined with it, the amounts retained by the butter are quite variable in different churnings.

213. Variations in the composition of butter. As an illustration of the variations in the composition of butter that usually occur, the analyses made in the breed tests at the World's Fair in 1893 may be here cited; the butter was in all cases made by as nearly identical methods and under as uniform conditions as could possibly be obtained by the skilled operators having this work in charge; the average composition of 350 samples of this butter, with upper and lower limits, was as shown in the following table:

Composition of samples of butter, World's Fair, 1893.

\begin{tabular}{|c|c|c|c|c|c|}
\hline & Water & Fat & Curd & $\begin{array}{l}\text { Salt and } \\
\text { ash }\end{array}$ & $\begin{array}{c}\text { Sum of } \\
\text { water, curd, } \\
\text { salt and } \\
\text { ash }\end{array}$ \\
\hline $\begin{array}{l}\text { Average of } 350 \\
\text { analyses }\end{array}$ & $\begin{array}{r}\text { Per cent. } \\
11.57\end{array}$ & $\begin{array}{r}\text { Per cent. } \\
84.70\end{array}$ & $\begin{array}{r}\text { Per cent. } \\
.95\end{array}$ & $\begin{array}{r}\text { Per cent. } \\
2.78\end{array}$ & $\begin{array}{r}\text { Per cent. } \\
15.30\end{array}$ \\
\hline $\begin{array}{l}\text { Lower and up- } \\
\text { per limits }\end{array}$ & $8.63-15.00$ & $76.53-88.26$ & $.50-2.14$ & $1.01-8.58$ & \\
\hline
\end{tabular}

Analyses of fifty samples of creamery butter taken in 1896, from the tubs ready for market at as many Wisconsin creameries, showed that no two of them were exactly alike in composition, but varied within the limits given on the following page : ${ }^{1}$

1 Wisconsin experiment station, bull. 56. 
Summary of analyses of Wisconsin creamery butter.

\begin{tabular}{|c|c|c|c|c|c|}
\hline & Water & Fat & Curd & $\begin{array}{l}\text { Salt and } \\
\text { ash }\end{array}$ & $\begin{array}{c}\text { Sum of } \\
\text { water, curd, } \\
\text { salt and } \\
\text { ash }\end{array}$ \\
\hline $\begin{array}{l}\text { Highest } \\
\text { Lowest ... } \\
\text { A verage }\end{array}$ & $\begin{array}{c}\text { Per cent. } \\
17.03 \\
9.18 \\
12.77\end{array}$ & $\begin{array}{c}\text { Per cent. } \\
87.50 \\
77.07 \\
83.08\end{array}$ & $\begin{array}{c}\text { Per cent. } \\
2.45 \\
.36 \\
1.28\end{array}$ & $\begin{array}{c}\text { Per cent. } \\
4.73 \\
1.30 \\
2.87\end{array}$ & $\begin{array}{c}\text { Per cent. } \\
22.95 \\
12.50 \\
16.92\end{array}$ \\
\hline
\end{tabular}

The preceding analyses show the composition of butter made at one place where every possible effort was taken to produce a uniform product, and of butter made at fifty different creameries, where there was more or less variation in the different operations of manufacture and in the appliances and machinery used. The majcrity of the samples of butter analyzed, in either case, were very near the average composition given, but since there are such wide variations in the composition of the butter made by the uniform methods adopted in the World's Fair breed tests, butter of a more uniform composition cannot be expected from the thousands of different creameries and private dairies which supply the general market with butter.

The analyses of the fifty samples of ereamery butter, given above, show that the content of the butter fat varied from 77 to 87.5 per cent., and according to the average of the analyses, 83 pounds of butter fat was contained in, or made, $100 \mathrm{lbs}$. of butter. There was, therefore, in this case produced 20.5 per cent. more butter than there was butter fat, since

$$
\begin{aligned}
83: 100:: 100: x ; \text { therefore } \\
x=\frac{100 \times 100}{83}=120.5 .
\end{aligned}
$$


214. "Overrun" of churn over test. The yield of butter is not, however, as a rule compared with the amount of butter fat contained in the butter, but with the total butter fat of the whole milk or cream from which it was made. This "increase of the churn over the test" is what is generally called overrun in creameries.

The overrun obtained in different creameries, or even in the same creameries at different times, will be found to vary considerably. When the milk is accurately tested and the butter well worked, this overrun will vary from 10 to 16 per cent.; that is, if a quantity of milk contains exactly $100 \mathrm{lbs}$ of butter fat, as found by the Babcock test or any other accurate method, from 110 to $116 \mathrm{lbs}$. of butter ready for market will be obtained from it. The overrun from cream will be somewhat larrger, 18 to 22 per cent., but will never exceed 25 per cent., unless the butter contains less than 80 per cent. fat (217).

2r5. Factors influencing the overrun from milk. Even under the very best of care and attention to details, variations will occur in the speed of the separator, in the conduct of the ripening and churning processes, and in the condition of the butter when the churn is stopped; hence absolutely uniform losses of fat in skim milk and butter milk, or the same water- and salt contents of the butter, cannot be expected.

The overmun in separator creameries is influenced by two legitimate factors: first, the losses of butter fat sustained in separating the milk and churning the cream, and second, the gain due to the admixture of water, 
salt, etc., in the manufacture of butter. Considering first the losses of fat in skim milk and butter milk, the separator will usually, when run at normal speed and capacity, leave the same per cent. of fat in skim milk, whether rich or poor milk is skimmed. An exception to this may be found in separating rich milk having large fat globules, or milk from fresh milkers, in either of which cases the large size of the fat globules occasions a more complete separation of fat by the centrifugal force. But generally speaking, the statement holds good that the total loss of fat in separator skim milk is a factor of the quantity of milk run through the separator, rather than of its quality.

2I6. The losses from poor, rich and average milk, as received at creameries and cheese factories, can be traced from the following statement; this gives the quantities of fat lost in handling milk of four grades, viz.: 2.5, $3.5,4.0$ and 6.0 per cent., in case of each grade calculated to a standard of $100 \mathrm{lbs}$. of fat in the milk.

To supply $100 \mathrm{lbs}$. of fat would require the following amounts of the different grades of milk:

4000 lbs. of milk testing 2.5 per cent will contain $100 \mathrm{lbs}$. of fat. 2857

2500 66603.5

$66 \quad 66$

100 1666

$66 \quad 66$

666666 666

6.0

$66 \quad 6$

$10066 \quad 66 \quad 66$

Assuming that the skim milk contains .1 per cent. of fat and makes up 85 per cent. of the whole milk, and that the butter milk tests .3 per cent., and forms 10 per cent. of the whole milk, the butter-fat record of the quantities of different grades of milk containing 100 lbs. of fat will be as given in the following table. Cer- 
tain mechanical losses are unavoidable in the creamery, as in all other factory operations, viz., milk and cream remaining in vats and separators, butter sticking to the walls of the churn, etc. These losses have been found to average about 3 per cent. of the total fat in the milk handled, under normal conditions and under good management (219).

Fat available for butter in different grades of milk.

\begin{tabular}{|c|c|c|c|c|c|c|}
\hline Grade of milk & $\begin{array}{l}\text { Whole } \\
\text { milk }\end{array}$ & $\begin{array}{l}\text { Skim } \\
\text { milk }\end{array}$ & $\begin{array}{l}\text { Butter } \\
\text { milk }\end{array}$ & 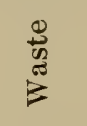 & $\begin{array}{l}\text { Total } \\
\text { loss }\end{array}$ & $\begin{array}{c}\text { Fat } \\
\text { available } \\
\text { for } \\
\text { butter }\end{array}$ \\
\hline 2.5 per cent.... & $\begin{array}{l}4000 \mathrm{lbs} \text {. } \\
2.5 \text { per } \mathrm{ct} \text {. }\end{array}$ & $\begin{array}{l}3400 \mathrm{lbs} \text {. } \\
.1 \text { per ct. }\end{array}$ & $\begin{array}{l}400 \text { lbs. } \\
.3 \text { per ct. }\end{array}$ & Lbs. & Lbs. & Per ct. \\
\hline Fat ... & $100 \mathrm{lbs}$ & $3.4 \mathrm{lbs}$ & $1.2 \mathrm{lbs}$ & 3.0 & 7.6 & 92.4 \\
\hline 3.5 per cent.... & $\begin{array}{r}2857 \mathrm{lbs} \text {. } \\
3.5 \text { per ct. }\end{array}$ & $\begin{array}{l}2429 \text { lbs. } \\
.1 \text { per ct. }\end{array}$ & $\begin{array}{l}286 \mathrm{lbs} \\
.3 \text { per ct. }\end{array}$ & & & \\
\hline Fat ........ & 100 lbs. & $2.4 \mathrm{lbs}$. & $.9 \mathrm{lb}$ & 3.0 & 6.3 & 93.7 \\
\hline 4.0 per cent $\ldots$ & $\begin{array}{l}2500 \mathrm{lbs} \text {. } \\
4 \text { per ct. }\end{array}$ & $\begin{array}{l}2125 \mathrm{lbs} \text {. } \\
.1 \text { per ct. }\end{array}$ & $\begin{array}{l}250 \mathrm{lbs} \text {. } \\
.3 \text { per ct. }\end{array}$ & & & \\
\hline Fat $\ldots . . .$. & $100 \mathrm{lbs}$. & $2.1 \mathrm{lbs}$ & $.7 \mathrm{lb}$ & 3.0 & 5.8 & 94.2 \\
\hline 6.0 per cent.. & $\begin{array}{l}1666^{2} / 3 \mathrm{lbs} . \\
6 \text { per ct. }\end{array}$ & $\begin{array}{l}1417 \text { lbs. } \\
.1 \text { per ct. }\end{array}$ & $\begin{array}{l}167 \mathrm{lbs} \text {. } \\
.3 \text { per ct. }\end{array}$ & & & \\
\hline Fat $\ldots$ & $100 \mathrm{lbs}$. & $1.4 \mathrm{lbs}$ & $.5 \mathrm{lb}$ & 3.0 & 4.9 & 95.1 \\
\hline
\end{tabular}

The table shows that with 2.5 per cent.-milk, there is a loss of $3.4 \mathrm{lbs}$. of fat in the skim milk, a loss of 1.2 lbs. of fat in the butter milk, and of $3.0 \mathrm{lbs}$. in the creamery waste, for every $100 \mathrm{lbs}$. of fat in the whole milk, or a total loss of 7.6 lbs. from these sources. In case of 6 per cent. milk these losses are $1.4 \mathrm{lbs}$., $.5 \mathrm{lb}$. and $3.0 \mathrm{lbs}$. for skim milk, butter milk and waste, respectively; a total loss of $4.9 \mathrm{lbs}$., or $2.7 \mathrm{lbs}$. less than the losses with poor milk. This difference in the losses 
shrinks to only .5 pound of fat in case of 3.5 and 4.0 per cent.-milk, when a quantity containing $100 \mathrm{lbs}$. of fat is handled in both cases.

The overrun from each of the four grades of milk can be calculated for butter containing a certain per cent. of fat. Assuming the fat content of butter to be 83 per cent. on the average (213), the quantity of butter obtained from the $100 \mathrm{lbs}$. of fat, or rather from the portion thereof which is available for butter, in each case will be as follows:

\begin{tabular}{|c|c|c|c|}
\hline $100 \mathrm{lbs}$ of fat from & $\begin{array}{l}\text { Available } \\
\text { fat }\end{array}$ & $\begin{array}{l}\text { Butter cont. } \\
83 \text { per ct. fat }\end{array}$ & Overrun \\
\hline $\begin{array}{l}4,000 \mathrm{lbs} \text {. of } 2.5 \text { per cent. milk } \\
2,857 \mathrm{lbs} \text {. of } 3.5 \text { per cent. milk } \\
2,500 \mathrm{lbs} \text {. of } 4.0 \text { per cent. milk } \\
1,666 \mathrm{lbs} \text {. of } 6.0 \text { per cent. milk }\end{array}$ & $\begin{array}{l}\text { Lbs. } \\
92.4 \\
93.7 \\
94.2 \\
95.1\end{array}$ & $\begin{array}{c}\text { Lbs. } \\
111.3 \\
113.0 \\
113.9 \\
114.6\end{array}$ & $\begin{array}{c}\text { Per ct. } \\
11.3 \\
13.0 \\
13.5 \\
14.6\end{array}$ \\
\hline
\end{tabular}

The overrun figures given above may be increased by saving some of the three pounds of butter fat lost by waste. If it were possible to entirely eliminate this loss there would be three pounds more available fat in each case, viz., 95.4, 96.7, 97.2, 98.1 lbs. These amounts of fat will make 115, 116.5, 117.1, and $118.2 \mathrm{lbs}$. butter: corresponding to an overrun of $15,16.5,17.1$, and $18.2 \%$ from milk of the different fat contents mentioned.

All butter makers should obtain more butter from a certain quantity of milk than the Babcnck test shows it to contain butter fat, but it is impossible to know exactly, except by chemical analysis, how much butter fat is lost in the skim milk and the butter milk, and how much water, salt and curd the butter will contain. A 
butter maker's skill is shown by his ability to reduce the losses by waste in handling the milk, cream and butter, as well as the losses of butter fat in skim milk and butter milk, and his carefulness in weighing, sampling and testing the milk, cream and butter made.

2I7. Overrun from cream. The overrun from cream is, as already stated, larger than from milk because there is no loss of fat in the skim milk to be considered. Rich cream will give a slightly larger overrun than thin cream, for the same reasons as have been shown in the calculations of overrun from milk of different fat contents. If similar calculations are made for cream of different richness as those given above for milk, the fat available for butter-making and the yield of butter per 100 pounds of fat in the cream will be as shown below. A mechanical loss in the process of butter-making amounting to 2 per cent. has been assumed in these calculations:

\begin{tabular}{c|c|c|c}
\hline \hline $\begin{array}{c}100 \text { lbs. fat in } \\
\text { cream }\end{array}$ & Available fat & $\begin{array}{c}\text { Butter of } 83 \\
\text { per ct. fat }\end{array}$ & Overrun \\
\cline { 2 - 4 } Per cent & Lbs. & Lbs. & Per cent \\
20 & 96.8 & 116.6 & 16.6 \\
30 & 97.3 & 117.2 & 17.2 \\
40 & 97.6 & 117.6 & 17.6 \\
\hline
\end{tabular}

We note that the overrun for cream of different quality under the conditions given ranges from 16.6 for 20per cent. cream to 17.6 for 40 -per cent. cream. A somewhat larger overrun would be obtained when the butter made contains less fat and more water than assumed.

If no losses from waste are considered in the account, the figures for fat available for butter will be 98.8, 99.3, 
and $99.6 \mathrm{lbs}$, and the overruns when the butter contains $83 \%$ fat will be $19,19.3$, and 20 per cent. These overruns are higher than will be obtained under ordinary creamery conditions with butter containing 83.7 fat, because it is impossible to appreciably reduce the manufacturing losses in handling the cream and butter below 3 per cent.

217a. Maximum overrun for butter of a legal water content. If we assume that the butter contains the maximum amount of water allowed by law, viz., 16 per cent. (and therefore about 80 per cent fat), the overrun for both milk and cream would be somewhat larger than already given, as shown by the following figures :

\begin{tabular}{|c|c|}
\hline Maximum overrun from milk & Maximum orerrun from cream. \\
\hline 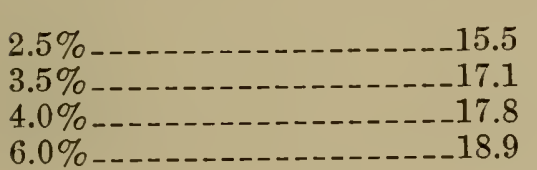 & $\begin{array}{l}20 \% \\
30 \%-21.0 \\
40 \%\end{array}$ \\
\hline
\end{tabular}

This table shows the highest overruns that are likely to be obtained when the butter is to contain no more than the maximum amount of water allowed by law. Larger overruns can only be obtained by reducing the losses of manufacture (which will give but slightly higher figures) or, fraudulently, by inaccurate weighing or testing of the milk, cream or hutter.

218. Calculation of overrun. The nverrun is calculated by subtracting the amount of butter fat contained in a certain quantity of milk or cream, from the amount 
of butter made from it, and finding what per cent. this difference is of the amount of butter fat in the milk.

Example 1: 8000 lbs. of milk is received at the creamery on a certain day; the average test of the milk is 3.8 per cent. By a simple multiplication we find that the milk contained $8000 \times$ $.038=304 \mathrm{lbs}$. of butter fat. $350 \mathrm{lbs}$. of butter was made from this milk, as shown by the weights of the packed tubs. The difference between the weight of butter and butter fat is, therefore, $46 \mathrm{lbs}$; 46 is $\frac{46 \times 101}{304}=15.1$ per cent. of the quantity of the butter fat in the milk; that is, the overrun for the day considered was 15.1 per cent.

The formula for the overrun is as follows:

$$
\mathrm{X}=\frac{(\mathrm{b}-\mathrm{f}) \mathbf{1 0 0}}{\mathrm{f}}
$$

$b$ and $f$ designating the quantities of butter and butter fat, respectively, made from or contained in a certain quantity of milk. In the preceding example, the calculation would be as follows: $\frac{(350-304) \times 100}{304}=15.1$ per cent.

Example 2: $1000 \mathrm{lbs}$. of cream testing 25 per cent. fat contains $1000 \times .25=250 \mathrm{lbs}$. butter fat. If $304 \mathrm{lbs}$. of butter is made, the overrun may be calculated by subtracting the butter fat from the butter, $304-250=54$ lbs., then divide this by the fat in the cream and multiply by 100 ; or $\frac{51 \times 100}{250}=21.8$ per cent., which is the cream overrun.

219. Conversion factor for butter fat. In the ninetyday dairy test at the World's Columbian Exposition, 96.67 per cent. of the fat in the whole milk was recovered in the butter. This butter, on the average, contained 82.37 per cent. butter fat; in other words, 117.3 pounds of butter were made from each 100 pounds of butter fat in the whole milk. ${ }^{1}$ The exact conversion factor

1 When $82.37 \mathrm{lbs}$. of butter fat will make $100 \mathrm{lbs}$. of butter, how wuch butter will 96.67 lbs. of butter fat make? $83.37: 96: 17:: 100: x$, $x=117.3$. 
would be 1.173. As this is an awkward number to use, and as $1 \frac{1}{6}$ is so nearly the same, it was recommended at the time that the approximate equivalent of butter be computed by multiplying the amount of butter fat by $11 / 6$, and this figure has been generally accepted for computing the yield of butter from a certain amount of butter fat in milk.

The figures given are the result of more than ordinary care in skimming, churning and testing, and probably represent the minimum losses of fat in the manufacturing processes. The increase of churn over test represented by one-sixth, or 16 per cent., may therefore be taken as a maximum "overrun" for milk under ordinary factory conditions.

220. Butter yield from milk of different richness. a. Use of butter chart. The approximate yield of butter from milk of different richness is shown in Table XI in the Appendix. This table is founded on ordinary creamery experience and will be found to come near to actual every-day conditions in creameries where modern methods are followed in the handling of the milk and its products. The table has been prepared in the following manner:

It is assumed that the average loss of fat in the skim milk is .20 per cent., and that $85 \mathrm{lbs}$. of skim milk is obtained from each 100 lbs. of whole milk; to this loss of fat is added that from the butter milk; about 10 lbs. of butter milk is obtained per 100 lbs. of whole milk, testing on the average .30 per cent.

If $f$ designate the fat in $100 \mathrm{lbs}$. of milk, then the fat recovered in the butter from 100 lbs. of milk will be

$$
\mathrm{f}-\left(\frac{8.5}{100} \times .20+\frac{10}{100} \times .30\right)=\mathrm{f}-.20
$$


There is, on the other hand, an increase in weight in the butter made, owing to the admixture of non-fatty components therein, principally water and salt. Butter packed and ready for the markat will contain in the neighborhood of 84 per cent. of fat (214), so that the fat recovered in the butter must be increased by ${ }_{84}^{100}=1.19$. If $B$ therefore designate the yield of butter from $100 \mathrm{lbs}$. of milk, the following formula will express the relation between yield and fat content, provided there are no other factors entering into the problem, viz.:

$$
\mathrm{B}=(\mathrm{f}-\mathrm{.20}) 1.19
$$

From this value for $B$, should be deducted the loss due to wastes in the manufacturing processes, amounting to 3 per cent. of the total fat in the milk handled, and we therefore have:

$$
\mathrm{B}=(\mathrm{f}-\mathrm{.20}) \quad 1.16
$$

Since this table is based on a fat content of .2 per cent. in the skim milk, the figures for the overrun are slightly lower than may be obtained in creameries provided with up-to-date cream separators.

221. Table XI in the Appendix, founded on this formula, may be used to determine the number of pounds of butter which mill containing 3 to 5.3 per cent. fat will be likely to make. It presupposes good and careful work in separating and churning and under such conditions will generally show yields of butter varying but little from those actually obtained. It may be conveniently used by the butter maker or the manager to check up the work in the creamery; the average test of the milk received during a certain period is found by dividing the total butter fat received, by the total milk, and multiplying the quotient by 100 ; the amount of butter which the total milk of this average fat content will make, according to the table, is then compared with the actual churn yield. 
Example: A creamery receives 200,000 lbs. of milk during a month; the milk of each patron is tested and the fat contained therein calculated. The sum of these amounts of fat may be $7583 \mathrm{lbs}$; the average test of the milk is then 3.79 per cent. According to Tabla XI, 10,000 lbs. of milk, testing 3.8, will make $418 \mathrm{lbs}$. of butter, and 200,000 lbs., therefore, $8360 \mathrm{lbs}$. of butter. The total quantity of butter made during the month will not vary appreciably from this figure if the work in the cream. ery has been properly done.

222. b. Use of overrun table. The table referred to above gives a definite calculated butter yield for each grade of milk, according to average creamery conditions. As it may be found that this table will give uniformly either too low or too high results, Table XII in the Appendix is included, by means of which the butter yield corresponding to overruns from 10 to 20 per cent. may be ascertained in a similar way as above described.

The total yield of butter is divided by the total num. ber of pounds of fat delivered; the quotient will give the amount of butter made from one pound of fat, and this figure multiplied by the fat delivered by each patron shows the pounds of butter to be credited to each patron. To use the table, find in the upper horizontal line the number corresponding nearest to the number of pounds of butter from one pound of fat. The vertical column in which this falls gives the pounds of butter from $100 \mathrm{lbs}$. of milk containing the per cents. of fat given in the outside columns (Babcock).

\section{B.-Calculation of Yield of Cheese.}

223. a. From fat. The approximate yield of green Cheddar cheese from $100 \mathrm{lbs}$. of milk may be found by multiplying the per cent. of fat in the milk by 2.7 : if $f$ 
designate the per cent. of fat in the milk, the formula will, therefore, be :

Yield of cheese $=2.7 \mathrm{f}$.

The factor 2.7 will only hold good as the average of a large number of cases. In extensive investigations during three consecutive years, Van Slyke ${ }^{x}$ found that the number of pounds of green cheese obtained for each pound of fat in the milk varied from 2.51 to 3.06 , the average figures for the three years 1892-'94, inclusive, being $2.73,2.71$, and $2.72 \mathrm{lbs}$., respectively. The richer kinds of milk will produce cheese richer in fat, and will yield a relatively larger quantity of cheese, pound for pound, than poor milk, for the reason that an increase in the fat content of milk is accompanied by an increase in the other cheese-producing solids of the milk. ${ }^{2}$ The preceding formula would not, therefore, be correct for small lots of either rich or poor milk, but only for milk of average composition, and for large quantities of normal factory milk. For cured cheese the factor will be somewhat lower, viz., about 2.6 , on the average.

224. b. From solids not fat and fat. If the percentages of solids not fat and of fat in the milk are known, the following formula by Babcock will give close results:

Yield of green cheese $=1.58\left(\frac{\mathrm{s}}{3}+.91 \mathrm{f}\right) \quad \ldots \quad$ - (II)

\footnotetext{
${ }^{1}$ N. Y. experiment station (Genera), bulletins 65 and S2.

${ }^{2}$ Investigations as to the relation between the quality of the milk and the yield of cheese have been conducted by a number of experiment stations; the following references give the main contributions published on this point; N. Y. (Geneva) exp. sta., reports 10-13, incl.; Wis. exp. sta., reports 11 and 12, bull. 197 ; Ont. Agr. College, reports 1894-'96, incl.; Minn. exp. sta., b. 19, reports 1892-'?4, incl.; Iowa exp. sta., bull. 21 ; Hoard's Dairyman, 1892, p. 2400.
} 
$s$ being the per cent. of solids not fat in the milk, and $f$ the per cent. of fat. ${ }^{1}$

The solids not fat can be readily ascertained from the lactometer reading and the per cent. of fat as shown in par. 120, by means of Table VI in the Appendix.

Table XIII in the Appendix gives the yield of cheese from $100 \mathrm{lbs}$. of milk containing from 2.5 to 6.0 per cent. fat, the lactometer readings of which range between 26 and 36. By means of this table cheese makers can calculate very closely the yields of cheese which certain quantities of milk will make; as it takes into consideration the non-fatty solids as well as the fat of the milk, the results obtained by the use of this formula will be more correct than those found by means of formula (I). The uncertain element in the formula lies in the factor 1.58, which is based on an average water content of 37 per cent. in the green cheese. This may, however, be changed to suit any particular case, e. g., 35 per cent. $\left(\frac{100}{65}=1.54\right), 40$ per cent. $\frac{100}{60}=1.67$, etc. The average percentages of water in green cheese found by Van Slyke in his investigations referred to above, were for the years 1892-'94, respectively, 36.41, 37.05 and 36.70 per cent.

225. c. From casein and fat. If the percentages of casein and fat in the milk are known, the yield of cheese may be calculated by the following formula, also prepared by Dr. Babcock:

Yield of cheese $=1.1 \mathrm{f}+2.5$ casein . . . . (III).

This formula will give fairly correct results, but no more so than formula (II) ; it is wholly empirical.

\footnotetext{
${ }^{1}$ For derivation of this formula, see Wisconsin experiment station, tweifth report, p. 105.
} 
Questions.

1. What is the average composition of American creamery butter, and between what extremes does the composition of butter vary?

2. What is the difference between the churn yield and the results obtained by the Babcock test?

3. What does the overrun represent?

4. Mention several factors that cause a large overrun.

5. Give an illustration of how the per cent. of increase of churn over test is found, and how the overrun is calculated.

6. Show by an example that butter containing $80 \%$ fat cannot give an overrun of more than $25 \%$.

7. How many pounds of butter containing $80 \%$ fat can be made from 100 lbs. fat?

8. Why is the overrun from cream greater than from milk?

9. What is the overrun when $70.5 \mathrm{lbs}$. of butter are made from $140 \mathrm{lbs}$. of milk, testing 3.15 per cent?

10. What is the overrun in each of the following cases 8 $220 \mathrm{lbs}$. butter from $8000 \mathrm{lbs}$. milk, testing $2.3 \%$ fat. 250 lbs. butter from 4000 lbs. milk, testing $5.8 \%$ fat. $600 \mathrm{lbs}$. butter from $2000 \mathrm{lbs}$. cream, testing $25.0 \%$ fat. $480 \mathrm{lbs}$. butter from $1000 \mathrm{lbs}$. cream, testing $40.0 \%$ fat.

11. How much butter containing (a) $80 \%$ fat, and (b) $82.5 \%$ fat can be made from $3250 \mathrm{lbs}$. milk, testing $4.3 \%$ fat, assuming that the skim milk is $80 \%$ of the whole milk and contains $0.1 \%$ fat, and the butter milk, which is the cream minus the fat, contains $0.25 \%$ fat? What is the overrun in each case?

12. How much butter is obtained from 5800 lbs. milk, testing $3.7 \%$ fat, when the overrun is (a) $12.5 \%$ and (b) $16 \%$ ?

13. Two cows in full milk produce, one $17.5 \mathrm{lbs}$. of milk a day, containing $4.35 \%$ fat; the other, $27.3 \mathrm{lbs}$. of milk, testing $3.4 \%$. If the milk of both is made into butter or cheese, how much butter or cheese may be expected from each one in a week?

14. What is a fair percentage of loss of fat by waste other than in skim milk and butter milk under average creamery conditions in case of milk and cream?

15. How much butter may be made from (a) 15,640 lbs. milk, testing $3.8 \%$ fat, and (b) $35,842 \mathrm{lbs}$. milk, testing $4.1 \%$ fat? (Use Table XI, Appendix.) 


\section{CHAPTER XIII.}

\section{CALCULATING DIVIDENDS.}

\section{A.-Calculating Dividends at Creameries.}

226. The simplest method of calculating dividends at creameries is to base the calculations on the amount of butter fat delivered by the various patrons. Each lot of milk is weighed when delivered at the creamery, and a small quantity thereof is saved for the composite sample, as previously explained under Composite Tests (180). Some creameries test these samples at the end of each week, and others after collecting them for ten days or two weeks. If the four weekly composite samples of a patron's milk tested 3.8, 4.0, 3.9, 4.1 per cent., these four tests are added together, and the sum divided by 4 ; the result, 3.95 per cent., is used as the average test of this milk. By multiplying the total number of pounds of milk delivered by this patron, by his average test, the total weight of butter fat in pounds delivered to the factory during the month is obtained. This weight of fat is then multiplied by the price to be paid by the creamery per pound of butter fat; the product shows the amount of money due this patron for the milk delivered during the time samples were taken.

227. Price per pound of butter fat. The method of obtaining the price to be paid for one pound of butter fat varies somewhat in different creameries, on account of the different ways of paying for the cost of manu- 
facturing the butter. The method to be followed is generally determined by agreement between the manufacturer and the milk producers, in case of proprietary creameries, or among the shareholders, in co-operative creameries. The following methods of paying for the cost of manufacture are at the present time in use in American creameries.

228. I. Proprietary creameries. First.-When the creamery is owned by some one person or company, the owner or owners agree to make the butter for about 3 cents a pound; the difference between the total receipts of the factory and the amount due the owner is then divided between the different patrons, according to the amount of butter fat contained in the milk which they delivered.

The price charged for making butter varies from $21 / 2$ to 4 cents per pound; the larger the amount of milk received at a factory, the lower will naturally be the cost of manufacturing the butter. ${ }^{1}$

Second.-The proprietor of the creamery sometimes agrees to pay a certain price for $100 \mathrm{lbs}$. of milk delivered, according to its fat content, the price of milk containing 4 per cent. of butter fat being the standard. This price may be changed during the different seasons of the year by mutual agreement.

Third.-A creamery owner may offer to pay 1 to 2 cents, usually $11 / 2$ cents, below the average market price of butter, for each pound of butter fat received in the milk.

${ }^{1}$ Bull. 56, p. 26, Wisconsin exp. station; see Report 1S, lowa state Dairy Commissioner, p. 33. 
229. II. Co-operative creameries. Where the creamery is owned by the patrons, one of the stockholders who is elected secretary attends to the details of running the factory and selling the product. His accounts show the amount of money received each month for the butter and other products sold, and the expenses of running the factory during this time. The expenses are subtracted from the receipts, and the balance is divided among the patrons, each one receiving his proportionate share according to the amounts of butter fat delivered in each case, as shown by the total weight and the average test of milk delivered during this time.

In nearly all cases, the farmers receive about eighty pounds of skim milk for each one hundred pounds of whole milk they deliver to the factory, in addition to the amount received for the milk, calculated according to one or the other of the preceding methods.

230. Illustrations of calculation of dividends. In order to illustrate the details of calculating dividends, or the amount to be paid each patron for the milk delivered, when payments are made by each of the four systems given, it will be assumed that a creamery receives 5000 pounds of milk daily during a month, and makes $6650 \mathrm{lbs}$. of butter from the 150,000 lbs. of milk received during this time. The average test of this milk may be found by multiplying the total weight of milk delivered by each patron by his average test, and dividing the sum of these products by the total weight of milk received at the creamery (in the example given, by 150,000), the quotient being multiplied by 100 . Such ealculations may show that, e. g., $5700 \mathrm{lbs}$. of butter fat have been received in all the milk delivered by the different patrons; this multiplied by 100 and divided by 150,000 gives 3.8 as the average test, or the average amount of butter fat in each $100 \mathrm{lbs}$. of milk received during the month.

So far the method of calculation is common for the different systems of payment given above; the manner of procedure now 
differs according to the agreement made between owner and patrons, or among the shareholders, in case of co-operative creameries.

231 I. First.-If the net returns for the $6650 \mathrm{lbs}$. of butter 'sold during the month were $\$ 1197$, and the creamery is to receive 4 cents per pound of butter as the cost of manufacture, etc., the amount due the creamery is $6650 \times .04=\$ 266$, and the patrons would receive $\$ 1197-\$ 266=\$ 931$. This sum, $\$ 931$, is to be paid to the patrons for the 5700 lbs. of butter fat, which, as shown above, was the weight of fat contained in the $150,000 \mathrm{lbs}$. of milk delivered during the month. The price of one pound of butter fat is then easily found: $\$ 931 \div 5700=161 / 3$ cents. This price is paid to all patrons for each pound of butter fat delivered in their milk during the month. The monthly milk record of three patrons may, e. g., be as given in the following table:

\begin{tabular}{|c|c|c|c|c|c|c|c|c|c|c|}
\hline \multirow{2}{*}{ Patron } & \multicolumn{2}{|c|}{$\begin{array}{l}\text { First } \\
\text { week }\end{array}$} & \multicolumn{2}{|c|}{$\begin{array}{l}\text { Second } \\
\text { week }\end{array}$} & \multicolumn{2}{|c|}{$\begin{array}{l}\text { Third } \\
\text { week }\end{array}$} & \multicolumn{2}{|c|}{$\begin{array}{c}\text { Fourth } \\
\text { week }\end{array}$} & \multirow{2}{*}{$\begin{array}{l}\text { Total } \\
\text { Milk }\end{array}$} & \multirow{2}{*}{ 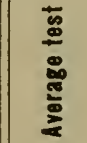 } \\
\hline & Milk & Test & Milk & Test & Milk & Test & Milk & Test & & \\
\hline & Lbs. & $\%$ & Lbs. & $\%$ & Lbs. & $a_{c}$ & Lbs. & $\%$ & Lbs. & $\%$ \\
\hline $\begin{array}{l}\text { No. } 1 \text {... } \\
\text { No. } 2 \ldots\end{array}$ & $\begin{array}{r}3500 \\
700\end{array}$ & $\begin{array}{l}3.6 \\
3.8\end{array}$ & $\begin{array}{r}3000 \\
665\end{array}$ & $\begin{array}{l}3.5 \\
3.8\end{array}$ & $\begin{array}{r}3600 \\
720\end{array}$ & $\begin{array}{l}3.65 \\
3.6\end{array}$ & $\begin{array}{r}3450 \\
750\end{array}$ & $\begin{array}{l}3.45 \\
3.7\end{array}$ & $\begin{array}{r}13,550 \\
2,825\end{array}$ & $\begin{array}{l}3.55 \\
3.73\end{array}$ \\
\hline No. 3 & 2480 & 4.2 & 2000 & 3.8 & 1850 & 4.0 & 1500 & 3.6 & 7,830 & 3.90 \\
\hline
\end{tabular}

Multiplying each patron's total milk by his average test gives the number of pounds of butter fat in his milk, and this figure multiplied by $.16 \frac{1}{3}$ shows the money due for his milk, as given below:

\begin{tabular}{|c|c|c|c|c|c|}
\hline Patron & $\begin{array}{l}\text { Total } \\
\text { milk }\end{array}$ & $\begin{array}{c}\text { Average } \\
\text { test }\end{array}$ & $\begin{array}{c}\text { Butter } \\
\text { fat }\end{array}$ & $\begin{array}{c}\text { Price of fat } \\
\text { per lb. }\end{array}$ & $\begin{array}{c}\text { Amount } \\
\text { due }\end{array}$ \\
\hline $\begin{array}{l}\text { No. } 1 \\
\text { No. } 2 \ldots \\
\text { No, } 3 \ldots\end{array}$ & $\begin{array}{r}\text { Lbs. } \\
13,550 \\
2,835 \\
7,830\end{array}$ & $\begin{array}{c}\text { Per cent } \\
3.55 \\
3.7 \\
3.9\end{array}$ & $\begin{array}{c}\text { Lbs. } \\
481.0 \\
104.5 \\
305.4\end{array}$ & $\begin{array}{l}\text { Cents } \\
16^{1} / 3 \\
16^{1 / 3} \\
16^{1 / 3}\end{array}$ & $\begin{array}{r}\$ 78.56 \\
17.06 \\
48.87\end{array}$ \\
\hline
\end{tabular}

232. Second.-When the proprietor of a creamery agrees to pay a certain price for $100 \mathrm{lbs}$. of 4 per cent. milk, the receipts for butter sold and the price per pound of butter do not enter into the calculation of the amount due each patron for his milk; 
but the weight and the test of each patron's milk are as important as before. If it is agreed to pay 66 cents per $100 \mathrm{lbs}$. of 4 per cent. milk (i. e., milk containing 4 per cent. of butter fat), the price of one pound of butter fat will be $66 \div 4=161 / 2$ cents, and the arnount due each patron is found by multiplying the total weight of butter fat in his milk by this price. To facilitate this calculation, so-called Relative-Value Tables have been constructed, the use of which is explained below (238).

233. Third.-If a creamery agrees to pay for butter fat, say $11 / 2$ cents per pound below the average market price of butter each month, the price of one pound of butter fat is found by averaging the market quotations and subtracting $11 / 2$ cents therefrom. If the four weekly market prices were $171 / 2,17,161 / 2$ and 19 cents, the average of these would be $171 / 2$ cents, and this less $11 / 2$ gives 16 cents as the price per pound of fat to be paid to the patrons; this price is then used in calculating the dividend as in case of first method (231).

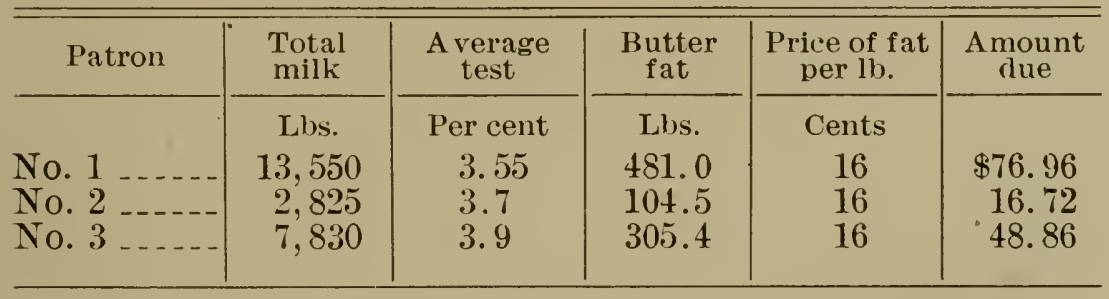

234. II. If the creamery is owned by the farmers, the running expenses for a month are subtracted from the gross returns received for the butter, and the price to be paid per pound of butter fat is found by dividing the amount left by the total number of pounds of butter fat delivered during the month. This price is used for paying each patron for his milk according to the amount of fat contained therein, as already explained under Proprietary Creameries (231).

The monthly running expenses of a co-operative creamery generally include such items as the wages of the butter maker (and manager or secretary, if these officers are salaried), labor (hauling, helper, etc.), cost of butter packages, coal or wood, salt and other supplies, freight and commission on the butter sold, repairs and insurance on buildings, etc. A certain amount is also paid into a sinking fund (say, 5 cents per $100 \mathrm{lbs}$. of milk), 
which represents the depreciation of the property, wear and tear of building and machinery, bad debts, etc. Thesel items are added together, and their sum subtracted from the gross receipts for the butter sold during the month.

235. Assuming the receipts for the butter during the month to be $\$ 1197$, and the running expenses of the factory $\$ 285$, the amount to be divided among the patrons is $\$ 912$; the quantity of butter fat received was 5700 lbs., and the price per pound of butter fat will therefore be 16 cents. The account will then stand as given in (233).

236. Other systems of payment. Besides these four systems of payment, there are various other agreements made between manufacturer and producer, but with them all the one important computation is the price to be paid per pound of butter fat; this forms the basis of calculating the factory dividends, when milk is paid for by the Babcock test.

237. Paying for butter delivered. In some instances patrons desire to receive pay for the quantity of butter which the milk or cream delivered by them would make. This can be ascertained quite satisfactorily from the total receipts and the total weights of both butter fat and butter. The total money to be paid for butter (the net receipts) are divided by the number of pounds of butter sold, to get the price to be paid per pound of butter; the total yield of butter divided by the total amount of butter fat delivered in the milk, gives the amount of butter corresponding to one pound of butter fat, and the number of pounds of fat delivered by each patron is then multiplied by this figure. This method requires more figuring than those given in the preceding, and the dividends are no more accurate, in fact less so, than when calculations are based on the price per pound of fat. 
237a. Making butter "for the overrun." When cream is bought on the basis of paying the market price of butter for each pound of butter fat in the cream, the margin received by the cream buyer, if he makes this cream into butter, is influenced both by the price of butter and the per cent. of overrun he obtains. If the price of butter is 20c. and the overrun is $20 \%$, each pound of butter fat makes $1.2 \mathrm{lbs}$. of butter, and the buyer receives 24 cents for the butter, or 4 cents margin on the $1.2 \mathrm{lbs}$. of butter made, which is equal to $3 \frac{1}{3}$ cents per pound of butter. If the price of butter is 36 cents, and the overrun $20 \%$, the cream buyer receives $1.2 \times 36=43$ cents for the butter, or 7 cents for $1.2 \mathrm{lbs}$. of butter, equivalent to $5.8 \mathrm{c}$. per pound of butter.

238. Relative-value tables. These tables give many of the multiplications used in computing the amount due for various weights of milk of different fat contents. They can easily be constructed by any one as soon as the price of one pound of fat is determined in each case. If the price to be paid per pound of fat is, say 25 cents, the value of each 100 lbs. of milk of different quality is found by multiplying its test by 25 . If the average tests of the different patrons' milk vary from 3 to 5 per cent., the relative-value table would be as follows.

$3.0 \times 25=75 \mathrm{c}$. per $100 \mathrm{lbs}$.
$3.1 \times 25=77.5 \mathrm{c}$.
$3.2 \times 25=80 \mathrm{c}$.
$3.3 \times 25=82.5 \mathrm{c}$.
$3.4 \times 25=85.0 \mathrm{c}$.
$3.5 \times 25=87.5 \mathrm{c}$.

$3.6 \times 25=90$ c. per $100 \mathrm{lls}$.

$3.7 \times 25=92.5 \mathrm{c}$.

$3.8 \times 25=95.0 \mathrm{c}$.

$3.9 \times 25=97.5 \mathrm{c}$.

$4.0 \times 25=100 \mathrm{c}$. etc.

By continuing this multiplication, or adding the multiplier each time for each tenth of a per cent. up to 5 per cent. of fat, a table is made that can be used for 
calculating the amount due per 100 lbs. of milk at the price per pound given, and the weight of milk delivered by each patron is multiplied by the price per $100 \mathrm{lbs}$. of milk shown in the table opposite the figure representing his test.

Example: A patron supplies 2470 lbs. of milk, testing 3.2 per cent. of fat; price per pound of fat, 25 cents; he should then receive $24.70 \times .80=\$ 19.76$ (see above table). Another patron delivering $3850 \mathrm{lbs}$. of milk testing 3.8 per cent. will receive, at the same price per pound of fat, $38.50 \times .95=\$ 35.57$.

The relative-value tables in the Appendix give the price per $100 \mathrm{lbs}$. of milk testing between 3 and 6 per cent. fat, when the price of three per cent. milk varies from 30 to 90c. per 100 lbs. In using the tables, first: find the figure showing the price which it has been determined to pay for $100 \mathrm{lbs}$. of milk of a certain quality, say 3 or 4 per cent.-milk; the figures in the same vertical column then give the price to be paid per 100 lbs. of milk testing between 3 and 6 per cent.

Example 1: It has been decided to pay 90 cents per 100 lbs. of 4 per cent.-milk. The figure 90 is then sought in the table in the same line as 4.0 per cent., and the vertical column in which it is found gives the price per $100 \mathrm{lbs}$. of 3 to 6 per cent.-milk; 3.8 per cent.-milk is thus worth 85 cents per $100 \mathrm{lbs}$. and 4.5 per cent.-nilk, $\$ 1.01$, under the conditions given. The prices of milk of other qualities are found in the same way.

Example 2: In the example referred to under Illustrations of calculating creamery dividends ( $I \mathrm{~b}, 231$ ), the figures for the patrons Nos. 1, 2 and 3, would be as follows:

\begin{tabular}{|c|c|c|c|c|}
\hline Patron & Mill delivered & $\begin{array}{l}\text { A verage } \\
\text { test }\end{array}$ & $\begin{array}{c}\text { Price per } 100 \text { lbs. } \\
\text { of milk }\end{array}$ & Amount \\
\hline $\begin{array}{l}\text { No. } 1 \ldots \\
\text { No. } 2 \\
\text { No. } 3 \ldots \\
\end{array}$ & $\begin{array}{r}\text { Lbs. } \\
13,550 \\
2,825 \\
7,830\end{array}$ & $\begin{array}{l}\text { Per cent } \\
3.55 \\
3.7 \\
3.9\end{array}$ & $\begin{array}{l}\text { Cents } \\
58.5 \\
61.0 \\
64.0\end{array}$ & $\begin{array}{c}\$ 79.26 \\
17.23 \\
50.11\end{array}$ \\
\hline
\end{tabular}


239. Milk- and cream dividends. When cream from farm hand separators or other sources is brought to a factory receiving and skimming whole milk, the cream patron's dividend should be calculated a little differently than that of the milk patron (210).

In one case the dividend is based on the weight and the test of cream and in the other on the weight and the test of milk; the difference between the two being represented by the fat left in the factory skim milk. This skim milk fat is included in the milk patron's dividend and consequently ought also to be allowed for in calculating the amount due the cream patron. Such an allowance can be fairly made by multiplying the cream fat by 1.03. The amounts of fat thus obtained represent very nearly the fat in the milk from which the cream was skimmed and assumes that the fat returned to the milk patron in his skim milk is about three per cent. of the total fat in his whole milk.

Since both milk and cream patron suffer the same manufacturing losses in the butter milk, an equalization of the skimming losses is all that is necessary in order to put both on a uniform basis for calculating dividends.

240. The following illustration will help to make these calculations clearer. Milk patron No. 1 delivers to the creamery during the month $5320 \mathrm{lbs}$. of milk testing 3.8 per cent. fat, which therefcre contains $\left(\frac{5320 \times 3.8}{100}\right)=202$ lbs. butter fat. If the price paid the patrons is $20 \mathrm{e}$., then 202 multiplied by 20 amounts to $\$ 40.40$, the money due this patron for his milk. If another patron sent $485 \mathrm{lbs}$. of cream testing 22.0 per cent. fat to the same factory during the month, the weight of fat in the cream is first found in the same way as in the milk. $\left(\frac{485 \times 22}{100}\right)=106.7 \mathrm{lbs}$. but- 
ter fat. Now, instead of multiplying this butter fat by $20 \mathrm{c}$, as was done for the whole milk patron, it must first be multiplied by 1.03 . $106.7 \times 1.03=109.9$ lbs. butter fat which is now multiplied by 20c. per pound, giving $\$ 21.98$. This is the amount due the cream patron when both milk and cream are received at the same factory and the cream from both patrons is churned together. ${ }^{1}$

24r. The amount of cheese made from a certain quantity of milk depends, as before shown, in a large measure on the richness of the milk in butter fat (223). Rich milk will give more cheese per hundred weight than poor milk, and within the ordinary limits of normal factory milk the increased yields will be nearly, but not entirely, proportional to the fat contents of the different kinds of milk. Since the quality of the cheese produced from rich milk is better than that of cheese made from thin milk and will demand a higher price, it follows that no injustice is done by rating the value of milk for cheese production by its fat content. This subject was discussed frequently during the nineties in experiment station publications and in the dairy press (223). Among others, Babcock has shown that the price of cheese stands in a direct relation to its fat content. ${ }^{2}$ Prof. Robertson, ex-Commissioner of Agriculture of Canada, is authority for the statement that the quality of the cheese made from milk containing 3.0 to 4.0 per cent. of fat was increased in value by one-eighth of a cent per pound for every two-tenths of a per cent. of fat in the milk, ${ }^{3}$ a figure which is fully corroborated by

\footnotetext{
117 th report Wis. exp. station, p. $90 ; 20$ th report, pp. 130-131.

2 Wisconsin exp. station, 11th report, p. 134.

${ }^{3}$ Hoard's Dairyman, Mal'ch 29, 1895.
} 
Dr. Babeock's results. The injustice of the "pooling system," by which all kinds of milk receive the same price, is evident from the preceding; if the milk of a certain patron is richer than that of others, it will make a higher grade of cheese, and more of it per hundredweight; hence a higher price should be paid for it.

Payment on the basis of the fat content of milk is, therefore, the most equitable method of valuing milk for cheese making, and in case of patrons of cheese factories as with creamery patrons, dividends should be calculated on the basis of the results obtained by testing the milk delivered. The testing may be conveniently arranged by the method of composite sampling, in the way already described for creameries (180).

242. Cheese factory dividends. (a) Dividends based on fat test alone. As in the case of creameries. the price to be paid per pound of butter fat must first be ascertained. The factory records should show the number of pounds of cheese made from the total milk delivered to the factory during a certain time, generally one month, and the money received for this cheese. The cost of making the cheese and all other expenses that should be paid for out of the money received for the cheese, are deducted from the total receipts, and the difference is divided among the patrons in proportion to the amounts of butter fat delivered in the milk.

The weights of the milk delivered and the tests of the composite samples furnish data for calculating the quantities of butter fat to be credited to each patron. 
The money to be paid to the patrons is then divided by the total weight of butter fat delivered to the factory and the price of one pound of fat thus obtained. The money due each patron is now found by multiplying the total number of pounds of butter fat in his milk by this price per pound.

(b) Dividends based on fat and solids-not-fat (lactometer readings). A close estimate of the cheese value of each patron's milk may be made, as explained in par. 224, b., by the use of table XIII in the Appendix. When the cheese yield of each patron's milk is found by this method, the money to be distributed among the patrons is divided by the sum of the figures found by these two tests, instead by the total butter fat. The figure thus obtained is the price to be paid each patron per pound cheese that may be made from his milk as shown by both the fat test and the lactometer reading.

(c) Dividends based on "the fat plus two method." The money due patrons for milk delivered at cheese factories may be calculated by adding two to the per cent. of fat in the milk, and otherwise proceeding as explained above under par. 242a. This is the method advocated by Prof. H. H. Dean of Guelph (Ont.) Agricultural college. ${ }^{1}$ It has been adopted at many Canadian cheese factories and at some factories in this country.

(d) Dividends based on the fat and casein tests. The results obtained by the fat test and the Hart casein

${ }^{1}$ Bull. 114, Ont. Agr. College; see also Dean, Canadian Dairying, p. 146. 
test (258) are added together; the pounds of milk delivered by each patron are multiplied by this figure and the product multiplied by the price to be paid for the sum of the fat and the casein. ${ }^{1}$ This price per pound is obtained in the same way as the price per pound of fat. Each patron's milk is multiplied by the sum of the fat and the casein tests and the money to be distributed to the patrons is divided by the sum of these figures obtained for all patrons for the period covered.

The illustrations already given for calculating patrons' dividends at creameries according to the various methods will serve equally well to show the manner in which dividends are calculated at a cheese factory. For the sake of clearness an example is given that applies directly to cheese factories.

244. Illustration of calculation of dividends. It may be assumed that 15,000 $\mathrm{llbs}$. of green cheese is made from 150,000 lbs. of milk delivered to a factory in a month. According to the weighings and the tests made, the milk contained 5,700 lbs. of butter fat. If the cheese sold at an average price of $7 \frac{1}{2}$ cents a pound, the gross receipts would be $\$ 1,125.00$. The amount to be deducted from the gross receipts will depend on the agreement made between the factory operator and the patrons, in case of proprietary cheese factories, or between the shareholders and the maker, when the factory is run on the co-operative plan. As before we shall consider these systems separately.

244. I. Proprietary cheese factories. The owner of the factory generally agrees to make the cheese for a certain price per pound and to pay the patrons what is left after deducting this amount. If the price agreed on is $1 \frac{112}{2}$ cents per pound of green cheese, this would amount to $\$ 225$ in the example given. Subtracting this sum from the gross receipts, $\$ 1,125$, leaves $\$ 900$,

1 Visconsin expt. station, bull. 197. 
which is to be paid the patrons. The total amount of butter fat delivered by the patrons was 5,700 lbs.; hence the price of one pound of butter fat will be $900 \div 5,700=.1577$, or 15.8 cents. Taking the figures for the three patrons already mentioned under Creamery Dividends, we then have:

\begin{tabular}{c|c|c|c|c|c}
\hline \hline Patron & Total milk & $\begin{array}{c}\text { A verage } \\
\text { test }\end{array}$ & Butter fat & $\begin{array}{c}\text { Price per lb. } \\
\text { of fat }\end{array}$ & $\begin{array}{c}\text { Amount } \\
\text { due }\end{array}$ \\
\cline { 2 - 5 } \cline { 4 - 6 } & Lbs. & Per cent & Lbs. & Cents & \\
No. 1 & 13,550 & 3.55 & 481.0 & 15.8 & $\$ 76.00$ \\
No. 2 & 2,825 & 3.7 & 104.5 & 15.8 & 16.51 \\
No. $3 \ldots$ & 7,830 & 3.9 & 305.4 & 15.8 & 48.25 \\
\hline
\end{tabular}

245. II. Co-operative cheese factories. The method of payment at co-operative cheese factories is nearly the same as that already given, except that a certain sum representing the expenses is subtracted from the gross receipts for the cheese, and the balance is divided among the patrons according to the amount of butter fat furnished by each, in the same manner as in the above case, after the price of a pound of fat has been obtained.

The price per $100 \mathrm{lbs}$. of milk can be calculated in the same way as at creameries, by multiplying the test of each lot by the price per pound of fat. ${ }^{1}$

\section{Questions.}

1. How much money is due each of three patrons of a creamery when the following weights of milk are delivered by each:

A- 5750 lb. milk, composite tests, $4.0-4.8-4.2$ per cent.

B- $955 \mathrm{lb}$. milk, composite tests, $4.6-5.0-4.8$ per cent.

C-10,538 lb. milk, composite tests, $3.2-3.5-3.0$ per cent.

(a) When $700 \mathrm{lbs}$. of butter are sold for $\$ 200$, and the cost of making is $31 / 2 \mathrm{c}$. per $\mathrm{lb}$;

(b) When the factory agrees to pay $\$ 1.00$ per 100 lbs. milk, testing $4 \%$ fat;

${ }^{1}$ Suggestions regarding the organization of co-operative creameries and cheese factories will be found in the Appendix, following Table $\mathrm{XV}$. Draft of constitution and by-laws for co-operative factory associations are also given in the Appendix. It is hoped that these will prove helpful to farmers who contemplate forming such associations. 


\section{CHAPTER XIV.}

\section{CHEMICAL ANALYSIS OF MILK AND ITS PRODUCTS.}

246. An outline of the methods followed in determining quantitatively the main components of milk and its products is given in the following for the guidance of advanced dairy students. This work cannot be done outside of a fairly well-equipped chemical laboratory, or by persons who have not been accustomed to handling delicate chemical apparatus and glassware, analytical balances, etc., and who have not a knowledge of; at least, the elements of chemistry and chemical reactions.

\section{A.-Mrrk.}

247. In a complete milk analysis, the specific gravity of the milk is determined, and the following milk components: water, fat, casein and albumen, milk sugar, and ash. The methods of analysis described in the following are those adopted by the Association of Official Agricultural Chemists of North America, which, with but slight modifications, are in general use in the chemical laboratories of all American experiment stations and agricultural colleges. ${ }^{1}$

248. a. Specific gravity is determined by means of a picnometer or specific-gravity bottle, since more ac-

\footnotetext{
1 The complete methods of analysis adopted by the Association of Official Agricultural Chemists are published by the Bur. of Chemistry of the U. S. Department of Agriculture; see Bull. No. 107, pp. 117-128.
} 
curate results will thus be reached than by using an ordinary Quevenne lactometer. A thermoneter is ground into the neck of the specific-gravity bottle so as to form a stopper, and the bottle is provided with a glass-stoppered side-tube, to furnish an exit for the liquid on expanding. A specific-gravity bottle holding 100 grams of water is preferably used. The empty and scrupulously cleaned bottle is first weighed on a chemical balance. The bottle is then filled with recently-boiled distilled water of a temperature below $60^{\circ} \mathrm{F}$. (15.5 $5^{\circ} \mathrm{C}$.) ; the thermometer is inserted, and the bottle is warmed slightly by immersing it for a moment in tepid water and left standing until the thermometer shows $60^{\circ} \mathrm{F}$.; the opening of the side tube is then wiped off and closed with the stopper, and the water on the outside of the bottle and in the groove between its neck and the thermometer is wiped off with filter paper or a clean handkerchief, when the bottle is again weighed. The weight being recorded, the bottle is emptied and dried in a water oven, or if sufficient milk is at hand, the bottle is repeatedly rinsed with the milk, the specific gravity of which is to be determined. It is then filled with milk in a similar manner as in case of water; the temperature of the milk should be slightly below $60^{\circ} \mathrm{F}$. and is slowly brought up to this degree after the bottle has been filled, proceeding in the same way as before with water; the weight of the bottle and milk is then taken.

The weights of water and of milk contained in the specific-gravity bottle are found by subtracting the weight of the empty bottle from the second and the third weights, respectively, and the specific gravity of 
the milk then found by dividing the weight of the milk by that of the water.

Example: Weight of sp. gr. bottle+water...146.9113 grams.

Weight of sp. gr. bottle empty... 46.9423 grams.

Weight of water........ 99.9690 grams.

Weight of sp. gr. bottle+milk.....149.8708 grams.

Weight of sp. gr. bottle empty... 46.9423 grams.

Weight of milk........ $\overline{102.9285}$ grams.

Sp. gr. of milk $=\frac{102.9285}{99.969}=1.0296$.

249. If a plain picnometer without a themometer attached is available, the method of procedure is similar to that described, with the difference that the temperature of the water and of the milk must be bronght to $60^{\circ} \mathrm{F}$. before the picnometer is filled, or the picnometer filled with either liquid is placed in water in a small beaker, which is very slowly warmed to $60^{\circ} \mathrm{F}$. and kept at this temperature for some time so as to allow the liquid in the picnometer to reach the temperature desired; the temperature of the water in the beaker is ascertained by means of an accurate chemical thermometer. The perforated stopper is then wiped off, the picnometer is taken out of the water, wiped and weighed. It is necessary to weigh very quickly if the room temperature is much above $60^{\circ} \mathrm{F}$.: as in such cases the expanding liquid will flow on to the balance pan, with a resultant loss in weight from evaporation.

The weights of specific-gravity bottle or picnometer, empty and filled with water, need only be determined a couple of times, and the averages of these weighings are used in subsequent determinations.

250. Westphal balance. Where only a small amount of milk is available, or in rapid work, the specific gravity may be taken with considerable accuracy by means of a Westphal balance. The arrangement and use of this convenient little apparatus is readily explained verbally. 
For the determination of the specific gravity of loppered milk, see 263.

25I. b. Water. The milk is weighed into a perforated copper tube filled with prepared dry asbestos. The tubes are made from perforated sheet copper, with holes about $.7 \mathrm{~mm}$. in diameter and about $.7 \mathrm{~mm}$. apart; they are $60 \mathrm{~mm}$. long, $20 \mathrm{~mm}$. in diameter and closed at the bottom. The asbestos is prepared from clean fibrous asbestos, which is ignited at low heat in a muffle oven, treated with a little dilute $\mathrm{HCl}(1: 3)$ and then with distilled water till all acid is washed out; it is then torn in loose layers and dried at a low temperature in an air bath; when dry it can be easily shredded in fine strings and is placed in a wide-mouth, glass-stoppered bottle.

About two grams of asbestos are placed in each tube, packing it. rather loosely; the tube is then weighed, a small narrow beaker being inverted over it on the scale pan. 5 cc. of milk are now dropped on to the asbestos from a 5 cc. fixed pipette, the beaker again placed over the tube, and the weight of the 5 cc. of milk delivered +copper tube taken. The weight of the milk is obtained by difference. The tubes are then placed in a steam oven and heated at $100^{\circ} \mathrm{C}$. until they no longer decrease in weight, which will ordinarily take about three hours. Place in a desiccator until cold, and weigh; the difference between the weight of the tube+milk and this last weight gives the water contained in the milk, which is then calculated in per cent. of the quantity of milk weighed out. 
Chemical Analysis of Milk and Its Products. 221

Example: Weight of tube+beaker+milk...2 29.3004 grams. Weight of tube +beaker........ 24.1772 grams.

Milk weighed out....... 5.1232 grams. Weight of tube+beaker+milk... 29.3004 grams. Weight of tube+beaker+milk,dry 24.9257 grams.

Weight of water....... 4.3747 grams.

Per cent. of water in milk $=\frac{4.3747 \times 100}{5.1232}=\$ 539$ per cent.

Note. The per cent. of total solids in milk is often given, instead of that of water; this may be readily obtained by subtracting the weight of the empty tube from that of the tube filled with milk solids, and finding the per cent. of the milk weighed out which this difference makes. In the above example, the weight of milk solids thus is $24.9257-24.1772=.7485$ gram, and the per cent. of total solids in the milk $=14.61$ per cent.

252. Alternate Method. Five cc. of milk are measured out on a weighed flat porcelain dish $(50-60 \mathrm{~mm}$. in diameter; porcelain crucible covers will answer the purpose better than any other vessel on the market, if the handle be broken off or ground off level on an emery wheel); this is weighed rapidly; two or three drops of 30 per cent.-acetic acid are added, and the dish is dried in a steam oven at $100^{\circ}$ C. until no further loss in weight is obtained. After cooling in a desiccator, the weight of the milk solids is obtained, and by calculation as before, the per cent. of water or total solids in the milk.

253. c. Fat. The dried tubes from the water determination are placed in Caldwell extractors and connected with weighed, numbered glass flasks (capacity, 2-3 oz.) ; the extractors are attached to upright Liebig condensers and the tubes extracted with pure ether, free from water, alcohol or acid, until all fat is dissolved; $4-5$ hours' extraction is sufficient for whole milk; in case of samples of skim milk it is well to con- 
tinue the extraction for 8 hours. The ether is then recovered by distillation, and the flasks dried in a steam oven until constant weight; after cooling they are weighed and the amount of fat contained in the quantity of milk originally weighed into the tubes is thus ascertained, and the per cent. present in the milk calculated.

Example: Weight of flask+fat........ 15.8039 grams. Weight of flask........... 15.5171 grams.

Weight of fat......... .2868 gram.

Milk weighed out......... 5.1232 grams.

Per cent. of fat in milk $=\frac{.2868 \times 100}{5.1232}=5.60$ per cent.

254. The Gottlieb method for the determination of fat. ${ }^{1} 10$ cc. of milk are measured into a glass cylinder, $3 / 4$ inch in diameter and about 14 inches long (a 100 cc. burette or a Eudiometer tube will do); 1 cc. conc. ammonia is added and mixed thoroughly with the milk; the following chemicals are next added in the order given: 10 cc. of 92 per cent. alcohol, 25 cc. of washed ether, and 25 cc. petroleum ether (boiling pt., below $80^{\circ}$ C.), the cylinder being closed with a moistened cork stopper and the contents shaken several times after the addition of each chemical. The cylinder is then left standing for six hours or more. The clear fat solution is next pipetted off into a small weighed flask, by means of a siphon drawn to a fine point (see fig. 6, loc. cit.), which is lowered into the fat solution to within $1 / 2 \mathrm{~cm}$. of the turbid bottom layer. After evaporating the ether solution in a hood, the flasks are dried in a steam oven

${ }^{1}$ Landw. Vers. Sta., 40 (1892), pp. 1-27. The method is also spoken of as the Röse-Gootlieb method. 
for two to three hours, and weighed. This method is applicable to new milk, skim milk, butter milk, whey, cream, cheese, condensed milk and milk powder, but has been found of special value for determining fat in skim milk, butter milk, cheese, and condensed milk. In the case of products high in fat, a second treatment with $10 \mathrm{cc}$. each of ether and petroleum is advisable in order to recover the last traces of fat.

255. $d$. Casein and albumen. The sum of these components is generally determined by the Kjeldahl method. ${ }^{1} 5$ cc. of milk are measured carefully into a 800 cc. Jena flask, 20 cc. of concentrated sulfuric acid (C. P.; sp. gr., 1.84) are added, and .7 gram of mercuric oxid (or its equivalent in metallic mercury); the mixture is then heated over direct flame until it is straw-colored or perfectly white; a few crystals of potassium permanganate are now added till the color of the liquid remains green. All the nitrogen in the milk has then been converted into the form of ammonium sulfate. After cooling, 200 cc. of ammoniafree distilled water are added, 20 cc. of a solution of potassium sulfid (containing 40 grams sulfid per liter), and a fraction of a gram of powdered zinc. A quantity of semi-normal HCl-solution, more than sufficient to neutralize the ammonia obtained in the oxidation of the nitrogen in the milk, is now carefully measured out from a delicate burette (divided into $\frac{1}{2} \sigma$ ce.) into a receiving flask and the flask connected with a distillation apparatus. At the other end, the Jena flask containing

\footnotetext{
'Fresenius' Zeitschrift, 22, p. 366 ; O. S. Dept. Agr., Bur. of Chem., Bull. 107, p. 5 .
} 
the watery solution of ammonia sulfate is connected, after adding 50 cc. of a concentrated soda solution (1 pound "pure potash" dissolved in 500 cc. of distilled water and allowed to settle); the contents of the Jena flasks are now heated to boiling, and the distillation is continued for forty minutes to an hour, until all ammonia has been distilled over.

The excess of acid in the Erlenmeyer receiving-flask is then accurately titrated back by means of a tenthnormal standard ammonia-solution, using a cochinealsolution $^{1}$ as an indicator. From the amount of acid used, the per cent. of nitrogen is obtained; and from it, the per cent. of casein and albumen in the milk by multiplying by $6.25 .^{2}$ The amount of nitrogen contained in the chemicals used is determined by blank experiments and deducted from the nitrogen obtained as described.

Example: The weight of 5 cc. of milk (as obtained in determining the water in the milk) was 5.1465 grams. 5 cc. of stand ard $\mathrm{HCl}$ are added to the receiver, and 1.55 ce. of $\frac{10}{\mathrm{~N}}$ alkalisolution are used in titrating back the excess of acid. $1.55 \mathrm{cc}$. of $\frac{\mathrm{N}}{10}$ alkali $=\frac{1.55}{5}=.31$ cc. $\frac{\mathrm{N}}{2}$ acid solution; the ammonia distilled over therefore neutralized $5.00-.31=4.69$ cc. acid. By blank trials it was found that the reagents used furnished an equivalent of $.02 \mathrm{cc}$. acid in the distillate; this quantity subtracted from the acid-equivalent of the nitrogen of the milk leaves 4.67 ce. 1 cc. semi-normal HCl-solution corresponds to 7 milligrams or .007 gram of nitrogen; 4.67 cc. $\frac{\mathrm{N}}{2} \mathrm{HCl}$ therefore represents .03269 gram of nitrogen. The quantity of nitrogen was obtained from the 5.1465 grams of milk measured out; the milk therefore contains $\frac{.03269 \times 100}{5.1465}=.635$ per cent. of nitrogen, and $.635 \times 6.25=3.97$ per cent. of easein and albumen.

\footnotetext{
${ }^{1}$ Sutton, Volumetric Analysis, 4 th edition, p. 31.

2 The factor 6.30 or 6.37 is more correct for the albuminoids of milk, but has not yet been generally adopted (p. 15, foot note).
} 
256. Casein and albumen may be determined separately by Van Slyke's method; 10 grams of milk are weighed out and diluted with about 90 cc. of water at $40^{\circ}-42^{\circ}$ C. 1.5 cc. of a 10 per cent. acetic-acid solution are then added; the mixture is well stirred with a glass rod and the precipitate allowed to settle for 3 to $5 \mathrm{~min}$ utes. The whey is decanted through a filter and the precipitate washed two or three times with cold water. The nitrogen is determined in the filter paper and its contents by the Kjeldahl method; blank determinations with the regular quantities of chemicals and the filter paper used are made, and the nitrogen found therein deducted. The per cent. of nitrogen obtained multiplied by 6.25 gives the per cent. of casein in the milk.

257. Albumen is determined in the filtrate from the casein-precipitate; the filtrate is placed on a water bath and heated to boiling for a period of from ten to fifteen minutes. The washed precipitate is then treated by the Kjeldahl method for the determination of nitrogen; the amount of nitrogen multiplied by 6.25 gives the amount of albumen in the milk. The difference between the total nitrogenous components found by the Kjeldahl method, and the sum of the casein and the albumen, as given above, is due to the presence in milk of a third class of nitrogen compounds (18). ${ }^{2}$

257a. The protein of milk may also be obtained by calculation from the total solids of the milk by the use of the following formula worked out by Mr. Geo. A.

\footnotetext{
${ }^{1}$ Bulletin 107, p. 117, Bur. of Chem., U. S. Dept. of Agriculture.

2 Volumetric determinations of casein in milk have been proposed by Van Slyke and Bosworth (Geneva, N. Y.) expt. station, tech. bull. 10) and by Hart (Wis. expt. station, research bull. 11). 15 !
} 
Olson $^{1}: \quad \mathrm{P}=\mathrm{T}-\frac{\mathrm{T}}{1.34} \quad$ The results obtained by this formula are quite satisfactory. If we assume that .8 of the milk protein is casein, this component can also be obtained from the solids of the milk by a simple calculation by the use of the preceding formula.

258. Hart's test for casein in milk. The following test for casein in milk has been published by the Wisconsin experiment station. ${ }^{2}$

Two cc. of chloroform, 20 cc. of a .25 per cent. solution of acetic acid, and 5 cc. of milk (both these latter of a temperature of about $70^{\circ} \mathrm{F}$.) are measured into small tubes of special construction holding about 35 cc., the lower ends of which are narrow and graduated to .1 ce. The mixture is shaken for 10 to 20 seconds and the tubes then whirled $71 / 2$ or 8 minutes in a centrifuge of 15 inches diameter, making 2000 revolutions per minute. (The use of a metronome is recommended to facilitate the control of the speed.) After whirling, the tubes are taken out of the centrifuge and allowed to stand for 10 minutes, and the percentage of casein read off directly from the scale on the lower end of the tubes, each division of which represents .2 per cent. of casein when 5 cc. of milk are measured out. The test calls for considerable nicety of manipulation, but appears to give reliable results when the directions given are strictly followed. ${ }^{3}$

259. e. Milk sugar is generally determined by difference, the sum of fat, casein and albumen (totalN $\times 6.25$ ),

\footnotetext{
${ }^{1}$ Jouln. Ind. and Eng. Chemistry, I, 1909, p. 253.

2 Report 24, p. 117: "A simple method for the estimation of casein in cow's milk."

${ }^{3}$ See also Circ. 10. Wis. expt. sta., Operating the Casein Test at Cheese Factories.
} 
and ash, being subtracted from the total solids. It may be determined directly by means of a polariscope, or gravimetrically by Fehling's solution; only the former method, as worked out by Wiley, ${ }^{1}$ will be given here.

The specific gravity of the milk is accurately determined, and the following quantities of milk are measured out by means of a 100 cc. pipette graduated to .2 cc. (or a 64 cc. pipette made especially for this purpose, with marks on the stem between 63.7 and $64.3 \mathrm{cc}$.), according to the specific gravities given: $1.026,64.3 \mathrm{cc}$; $1.028,64.15$ ce.; $1.030,64.0$ cc.; $1.032,63.9$ cc.; 1.034 , 63.8 cc.; $1.036,63.7$ cc. These quantities refer to the Schmidt-Haensch half-shadow polariscopes, standardized for a normal weight of 26.048 grams of sugar. The milk is measured into a small flask graduated at $100 \mathrm{cc}$. and $102.6 \mathrm{cc}$; $30 \mathrm{cc}$. of mercuric-iodid solution (prepared from 33.2 grams potassium iodid, 13.5 grams mercuric chlorid, 20 cc. glacial acetic acid and 640 cc. water) are added; the flask is filled to 102.6 cc. mark with distilled water, the contents mixed, filtered through a dry filter, and when the filtrate is perfectly clear, the solution is polarized in a 200 millimeter tube. The reading of the scale divided by 2 , shows the per cent. of lactose (milk sugar) in the milk. Take five readings of two different portions of the filtrate, and average the results.

260. f. Ash. About 20 ce. of milk are measured into a flat-bottom porcelain dish and weighed; about one-half of a cc. of 30 per cent.-acetic acid is added, and the milk first dried on water bath and then ignited in a

\footnotetext{
I Aricultural Analysis, III, p. 275 ; Am. Chem. Jour., 6, p. 289 et seq.
} 
muffle oven at a low red heat. Direct heat should not be applied in determining the ash in milk, since alkali chlorids are likely to be lost at the temperature to which milk solids have to be heated to ignite all organic carbon.

Example: Weight of porcelain dish+milk... 49.0907 grams. Weight of porcelain dish....... 28.3538 grams.

Weight of milk........20.7369 grams. Weight of dish+milk, after ignition 28.5037 grams. Weight of dish............. 28.3538 grams.

Weight of milk ash....... .1499 gram. Per cent. of ash $=\frac{.1499 \times 100}{20.7369}=.72$ per cent.

The residue from the determination of solids by the Alternate Method given (252) may also be used for the ash determination.

B.-Cream, Skim milk, Butter milk, Whey, ConDENSED MILK.

262. The analysis of these products is conducted in the same manner as in case of whole milk, and the same constituents are determined, when a complete analysis is wanted. Skim milk, butter milk, and whey contain relatively small quantities of solids, and especially of fat, and it is, therefore, well to weigh out a larger quantity than in case of whole milk; if possible, toward 10 grams. The acidity of sour milk and butter milk must be neutralized with sodium carbonate previous to the drying and extraction, as lactic acid is soluble in ether and would thus tend to increase the etherextract (fat), if not combined with an alkali previous to the extraction. 
263. Specific gravity of butter milk. The specific gravity of butter milk (as well as of sour or loppered milk) is determined by Weibull's method; a known volume of the milk is mixed with a certain amount (say 10 per cent.) of ammonia of a definite specific gravity, and the specific gravity of the liquid determined after thorough mixing and subsequent standing for an hour. If $A$ designate the volume of butter milk taken, $B$ that of ammonia, and $C$ that of the mixture; and if furthermore $S$ designate the specific gravity of the butter milk, $s_{1}$ that of the ammonia, and $s_{2}$ that of the mixture, we have

$$
\frac{\mathrm{Cs}_{2}-\mathrm{Bs}_{1}}{\mathrm{~A}}
$$

Klein ${ }^{1}$ has modified this method by weighing the liquids, thus securing greater accuracy; 22 to 24 per cent.-ammonia is used, one-tenth as much being taken as the amount of milk weighed out. The results come uniformly .0005 too high, and this correction should always be made. The following formula will give the specific gravity of the milk, which in case of careful work will be accurate to one-half lactometer degree; if the letters given above designate weights (instead of volumes as before) and specific gravities of the liquids, respectively, we have

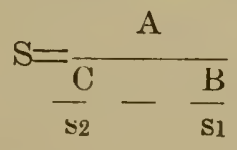

264. Condensed milk. The same methods are, in general, followed in the analysis of condensed milk as with whole milk. Condensed milk is preferably diluted with five times its weight of water prior to the analysis, both because such a solution can be more easily handled than the undiluted thick condensed milk, and the errors of analysis are thereby reduced, and because the fat ${ }^{2}$ is

${ }^{1}$ Milchzeitung, 1896 , p. 656 ; see also De Koningh, Analyst, 1899, p. 142.

${ }^{2}$ A second extraction following leaching and subsequent drying of the tubes is necessary to extract all the fat in condensed milk; see Bull. 104, Bur. of Chem., U. S. Dept. of Agr., p. 102 and 154. 
not readily extracted except when the milk has been diluted. The same ccustituents are determined as in case of whole milk, viz., solids, fat, casein and albumen, ash, milk sugar, and cane sugar (if any has been added to the milk). 'l'he cane sugar is determined by the difference between the solids not fat and the sum of the casein, albumen, milk sugar and ash; if the student has a knowledge of the manipulation of the polariscope and has had experience in gravimetric sugar analysis, the milk sugar is determined gravimetrically, and the cane sugar by the difference between the polariscope reading after inversion and the milk sugar present.

The specific gravity of condensed mill may be determined by a method similar to that of McGill. ${ }^{1} 50 \mathrm{gr}$. of the thoroughly mixed sample are weighed into a tared beaker and washed with warm water into a $250 \mathrm{cc}$. flask, cooled to $60^{\circ}$, filled to the mark and carefully mixed. The specific gravity of this solution $(a)$ is then taken and the original density is calculated by means of the following formula:

Sp. gr. of condensed milk $=\frac{1}{6-5 a}$

Concentration. The extent of concentration of condensed milk may be determined approximately by the formula devised by McGill (loc. cit.) :

\section{Concentration $(c)=\frac{a s}{\theta_{1} s_{1}}$}

where $a$ and $s$ designate the solids not fat and specific gravity, respectively, of the condensed milk, and $a_{1}$ and $s_{1}$ the corresponding data for the milk used. If $s_{1}=$ 1.030 and $a_{1}=9$ per cent., then $c=\frac{\text { as }}{9.27}$ gives the concentration.

\footnotetext{
${ }^{1}$ Bulletin 54, Laboratory Inland Rev. Dept., Ottawa, Canada.
} 
Chemical Analysis of Milk and Its Products. 231

\section{C. - Butter.}

265. Sampling. A four- to eight-ounce sample of butter is melted in a tightly-closed pint fruit jar, shaken vigorously and cooled until the butter is hardened, the jar being shaken vigorously at short intervals during the cooling so as to keep the water of the butter evenly distributed in the mass (102).

266. a. Determination of water. Small pieces of butter (about 2 grams in all) are taken from the sample by means of a steel spatula and placed in glass tubes, seven-eighths of an inch in diameter and two and a half inches long, closed at the bottom by a layer of stringy asbestos, and filled two-thirds full of asbestos prepared as for milk analysis (252). The tubes are dried at $100^{\circ} \mathrm{C}$. in a steam oven, until no further loss in weight takes place, and are then cooled and weighed. The loss in weight shows the per cent. of water present.

267. b. Fat. The tubes are placed in Caldwell extractors and extracted for four hours with anhydrous ether; the ether is then distilled off, and the flasks dried. in the steam bath and weighed, the increase in weight giving the fat in the sample of butter weighed out.

268. c. Casein. 10 grams of butter are weighed into a small beaker provided with a lip, and treated twice with about 50 cc. of gasoline each time; the solution is filtered off, and the residue transferred to a filter and dried; its nitrogen content is then determined by the Kjeldahl method (255). The nitrogen in the filter and the chemicals used is determined by blank trials and deducted. The nitrogen multiplied by 6.25 gives the casein in the butter. 
269. d. Ash. (1) 10 grams of butter are weighed into a porcelain dish and treated twice with gasoline, as in the preceding determination; the solution is filtered through an ash-free (quantitative) filter, and the filter when dry is transferred to the dish. The dish is heated in an air-bath for half an hour and then placed in a muffle oven, where the contents are burnt to a light grayish ash; the dish is now cooled in a desiccator and weighed. The difference between this weight and that of the empty dish gives the amount of ash in the butter weighed out.

270. (2) About two grams of butter are weighed into a small porcelain dish, half filled with stringy asbestos; the dish is dried for half an hour in the water oven, and the fat then ignited with a match, the asbestos fibre serving as a wick. When the flame has gone out. the dish is placed in a muffle oven, and the residue carefully burnt to a grayish ash. After cooling, the dish. is reighed, and the per cent. of ash in the butter calculated as under method 1.

271. Complete analysis of butter in the same sample. About 2 grams of the butter are weighed into a. platinum gooch half filled with stringy asbestos, and dried in a steam oven at $100^{\circ} \mathrm{C}$. to constant weight, cooled and weighed. The difference gives water in the sample. The gooch is then treated repeatedly with small portions of gasoline, suction being applied, and again dried in the water oven, cooled, and weighed; the fat in the sample is obtained from the difference between this and the preceding weight. The gooch is 
then carefully heated at a low red heat until a light grayish ash is obtained; this operation is preferably done in a muffle oven to avoid a loss of alkali chlorids. The loss in weight gives the casein in the sample weighed out, and the increase in the weight of the gooch over that of the empty gooch with asbestos, gives the ash (mainly salt) of the butter. The salt in the ash may be dissolved out by hot water, and the chlorin content of the solution determined by means of a standard silver-nitrate solution, using potassium chromate as an indicator (278).

\section{Creamery methods of estimating water in} butter. A number of different methods have been proposed of late years for the rapid estimation of water in butter, the object sought being to enable a buttermaker to a s e r tain the water content of his butter without much trouble or delay,

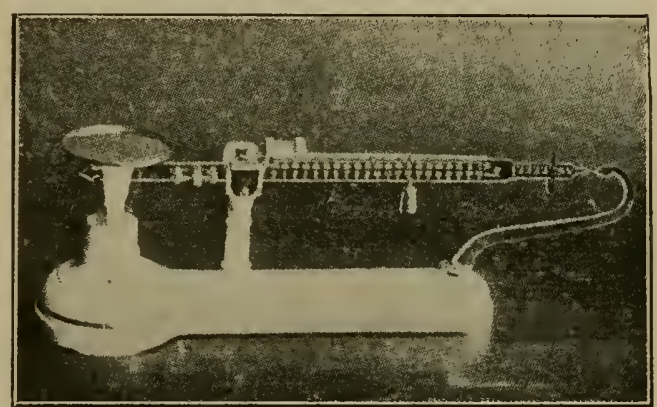

FIG. 58a. Balance for weighing butter for testing.

and by using such simple apparatus as he is likely to have in the creamery or can easily procure at a low price. The subject of controlling the per cent., of water in butter has become more important than was earlier the case, through the passage of the pure-food law, and the promulgation of government food standards in 1906 (305); these measures have rendered the question 
of guarding against an excessive water content in the butter one of the greatest importance to all buttermakers.

Most of the methods suggested for this purpose are essentially the common method of chemical analysis, modified to meet the demands of every-day factory con-

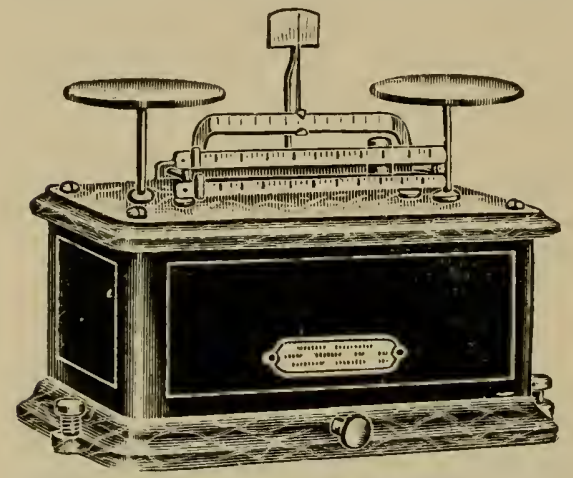

Fig. 58b. Scale for weighing butter for testing. ditions. References to descriptions of the different methods proposed are given below, and a few that are now used in factories and outside of chemical laboratories, are described in detail.

In all these rapid methods of determining the water content in butter, the sample of butter must be prepared so as to accurately represent the lot of butter sampled (see 102), and must be carefully weighed on a delicate scale (see figures $58 \mathrm{a}$ and $\mathrm{b}$ ). The directions, in so far as they are given in detail in the following, therefore, presuppose that a carefully prepared, fair sample has been obtained in all cases.

273. Among the methods proposed for the rapid determination of the per cent. of water in butter that are adapted for use in creameries may be mentioned:

Richmond's method, ${ }^{1}$ Carroll's tester, ${ }^{2}$ Geldard's but-

${ }^{1}$ Daily Chemistry, p. $225:$.

${ }^{2}$ Dept. of Agr., Ottawa, Dairy Com'r Branch, bull. 6, pp. 10-11. 
ter tester, ${ }^{1}$ the Irish "common sense butter and cheese test," Dean's, ${ }^{2}$ Gray's, ${ }^{3}$ Pitrick's, ${ }^{4}$ the Wisconsin high pressure oren method, ${ }^{5}$ the Ames method, ${ }^{6}$ and the Cornell moisture test. ${ }^{\top}$

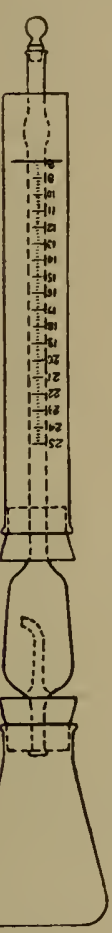

Fig. $5 y$. A pparatus used in Gray's method.

The following four of these methods will be briefly described:

274. a. Gray's method. This method, in. rented by Prof. C. E. Gray, formerly of the Dairy Division of the U. S. Dept. of Agriculture, was published in 1905; the method consists of heating ten grams of butter in a special flask of about 70 cc. capacity (see fig. 59 ) with 6 cc. of "amyl reagent" (five parts of amyl acetate and one part amyl valerianate). The water is boiled out of the butter by heating over direct flame, and together with some of the reagent, is condensed, cooled, and measured in a graduated tube attached to the flask. The accompanying illustration shows the arrangement of the distilling flask and the graduated tube in which the water is measured. For details of manipulation, reference is made to the original publication, or to the files of our dairy press published during 1906-7. ${ }^{\mathrm{s}}$

${ }^{1}$ Dept. of Agr., Ottawa, Dairy Com'r Branch, bull. 14, pp. 6-8.

2 Ontario Agr. College, rept. 1906, p. 120.

${ }^{3}$ Circ. 100, Bur. An. Ind., U. S. Dept. of Agr.

4 Journal Am. Chem. Soc., 28, 1906, p. 1611.

${ }^{5}$ Bull. 154, Wis. experiment station.

- Bull. 97, Iowa experiment station.

' Bull. 281, Cornell experiment station.

${ }^{8}$ E. g., New York Produce Review, Jan. 16, 1907; American Cheese Maker, Jan., 1907. 
A modification of the Gray method has been proposed by Mitchell and Walker of the Kingston (Ont.) Dairy School, and described as the Mitchell-Walker test. ${ }^{1}$

275. Patrick's method. Ten grams of butter are accurately weighed into a 300 cc. aluminum beaker (about 3 inches tall and 2 inches in diameter); this is held by means of a hand clamp over the flame of the alcohol lamp or a gas burner (see fig. 60) and very carefully heated until all the water is expelled. The beaker is then cooled by sinking it to the rim in water of $50^{\circ}$ to $60^{\circ}$, wiped dry, and the loss in weight calculated as water. If ten

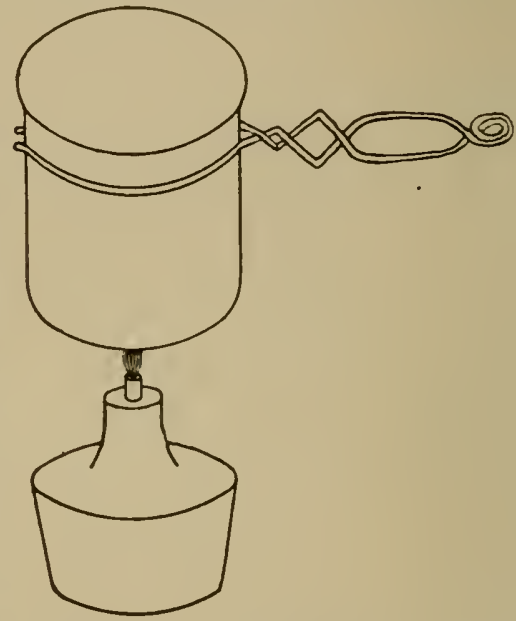
grams of butter weighed 8.45 grams after heating alcohal lamp used in the Patrick the loss in weight of 1.55 grams represents 15.5 per cent. of the weight of the sample, and the butter therefore contained 15.5 per cent. of water. ${ }^{2}$ The results obtained by this method seldom vary more than 2 per cent. from those of chemical analysis, and often less than .1 per cent. when proper care in sampling and weighing has been taken.

A few points need special attention in using this

${ }^{1}$ Bull. 167, Dairy Branch, Ont. Dept. of Agriculture.

$2 \mathrm{~A}$ convenient table showing per cents of moisture in butter direct when 9 to 10.15 grams are weighed out, has been published by the Copcnhagen experiment station (62nd report; see N. Y. Produce Rev.. 190 , p. 530). 
method: First, care must be taken not to heat the beaker too fast so that spattering occurs; there is not so much danger from this source when an alcohol lamp is used as with a gas burner, which easily raises the temperature too high, causing a fine spray of material to be thrown about, and thus giving too high results for water content. Second, it is important to discontinue the heating at the exact point when all the water has been driven off and before burning of the non-fatty solids (casein, milk sugar, and organic acids) occurs, as indicated by a slight darkening in color. It is not necessary to cool the beakers in water, but they can be left to cool in the air. The determination of water in butter by this method can be finished in ten minutes ur less by an experienced operator.

The Irish test is similar to the method described in the preceding, differing from the same mainly in the shape of the aluminum dishes used. Modifications of this test have also been worked out by the Iowa and Cornell experiment stations, which are designed to prevent losses by spattering when the dish is heated. In the Ames method the aluminum dish containing the sample is heated with a paraffine bath, while in the Cornell test a thin sheet of asbestos is placed between the flame and the dish holding the samples.

276. Dean's method. Three cc. of a melted sample of butter are placed in an ordinary "patty-pan" tin dish (about $21 / 2$ inches in diameter and $1 / 2$ inch deep) and accurately weighed; the dish is then placed in a steam oven provided with a pop safety valve, a steam 
pressure gauge, and a thermometer. The oven used by Professor Dean of Guelph (Ont.) Dairy School, the originator of this method, was $6 \times 8$ inches. It was made of galvanized iron by a local tin-smith at a cost of about $\$ 5.00$, exclusive of safety valve and steam gauge, and was made to withstand a pressure of about 10 pounds. After five or six hours' drying in the oven, the samples of butter are ready to be weighed, and the loss gives the amount of water present therein. The average results obtained by this method with nine samples of butter came within .13 per cent. of those found by chemical analyses.

The same method is recommended by the author for determining the per cent. of water in curd or cheese.

277. The Wisconsin high-pressure oven method (see fig. 61). Either 10 or 50 grams of butter are weighed in a flat-bottomed tin or aluminum dish. This is placed in an oven heated by high pressure steam to a temperature of $240^{\circ}$ to $280^{\circ} \mathrm{F}$. The length of time required to expel all the water from the butter will depend on the temperature of the oven and the diameter of the dish in which the butter is heated. If the dish is large enough to permit the butter to spread into a very thin layer and the temperature of the oven reaches $260^{\circ} \mathrm{F}$., the water will be completely expelled in half an hour. Ovens of this construction have now been placed on the market by manufacturers of dairy supplies. A steam pressure of $60 \mathrm{lbs}$. and a temperature of $280^{\circ} \mathrm{F}$. may be obtained in such an oven; by employing the boiler pressure ordinarily used in a 
creamery, temperatures of $240^{\circ}$ to $260^{\circ}$ may be easily obtained. The temperature thus reached is sufficient to dry the butter completely within an hour, provided pans large enough to spread the butter in a thin layer are used.

If 10 grams of butter are used in making tests, a more delicate scale is necessary than when 50 grams are taken. There are other advantages in using as large a quantity as 50 grams of butter for making tests of water. First, a sample can be weighed out directly from a package. Second, ordinary tin basins at least 5 inches in diameter can be used for drying the butter. Third, scales with a graduated side beam and sensitive to .1 gram instead of those with smaller loose weights can be used for weighing the butter. (See figs $58 \mathrm{a}$ and $58 \mathrm{~b}$.)

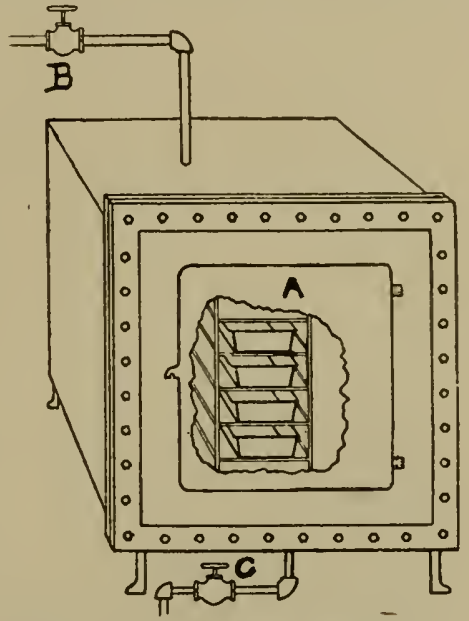

FIG. 61. The Wisconsin high. pressure oven.

278. Creamery methods of estimating salt in butter. T. The ordinary volumetric method used in chemical laboratories for determining the salt content of butter has been adapted for work in the creamery by Prof. Sammis. ${ }^{1} 5.1$ grams of chemically pure nitrate crystals are dissolved in 250 cc. of water. Each cc. of this solution will represent 1 per ct. of salt when 17.6 cc. of the liquid are measured which is obtained by shaking 10 grams of butter with $250 \mathrm{cc}$. of clean, warm water. The silver nitrate solution is added from a 25 or 50 cc. burette divided into tenths of a cubic centimeter. One or two drops of the usual indicator

${ }^{1}$ Circ. 14, Wisconsin expt. station. 
employed ( 1 oz. potassium chromate dissolved in 100 cc. of water) are added prior to the titration.

II. The use of silver nitrate tablets for making standard solutions for volumetric determinations of salt in butter was proposed.by Prof. Vivian and C. L. Fitch in 1901. ${ }^{1}$ During late years the tablets have not been on the market.

\section{Detection of Artificial Butter.}

279. Determination of the specific gravity of the filtered butter fat serves as a good preliminary test. A number of practical methods for the detection of artificial butter have been proposed, but "hey are either worthless for the examination of samples containing a considerable proportion of natural butter, or give satisfactory results only in the hands of experts. The Reichert-Wollny method given in detail below is the standard method the world over, and the results obtained by it are accepted in the courts.

280. Filtering the butter fat. The butter to be examined is placed in a small narrow beaker and kept at $60^{\circ} \mathrm{C}$. for about two hours. The clear supernatant fat is then filtered through absorbent cotton into a $200 \mathrm{cc}$. Erlenmeyer flask, taking care that none of the milky lower portion of the contents of the beaker be poured* on the filter. In sampling the butter fat, it is poured back and forth repeatedly from a small warm beaker into the flask, and the quantity wanted is then drawn off with a warm pipette.

281. Specific gravity. This is generally determined at $100^{\circ} \mathrm{C}$. The method of procedure is similar to that

\footnotetext{
1 Wis. experiment station, report 17, pp. 9S-101; Hoard's Dairyman, February 15, 1901, "Uniform Salting of Butter."
} 
described under milk (248). The picnometer (capacity about 25 cc.) is filled with dry filtered butter fat, free from air bubbles; the fat is heated for 30 minutes in a beaker, the water in which is kept boiling. On cooling, the weight of picnometer and fat is obtained, and by calculation as usual, the specific gravity of the fat.

The specific gravity of pure natural butter fat at $100^{\circ}$ C. ranges between .8650 and .8685 , while artificial butter fat (i. e., fat from other sources than cow's milk) has a specific gravity at $100^{\circ} \mathrm{C}$. of below .8610 , and generally about .85 .

282. Reichert-Wollny method (Volatile Acids.) 5.75 cc. of fat are measured into a strong 250 cc. weighed saponification flask, by means of a pipette marked to deliver this amount, and the flask when cool is weighed again. 20 cc. of a glycerol-soda solution (20 ce. of soda solution ( $1: 1)$ to $180 \mathrm{cc}$. of pure glycerol), are then added to the flask and the flask is heated over a naked flame or hot asbestos plate until complete saponification has taken place, as shown by the mixture becoming perfectly clear. If foaming occur, the flask is shaken gently.

135 cc. of recently-boiled distilled water are now added, drop by drop, at first, to prevent foaming, and when the solution is clear, cooled to about $70^{\circ} \mathrm{C}$.; 5 cc. of dilute sulfuric acid (200 cc. conc. $\mathrm{H}_{2} \mathrm{SO}_{4}$ per liter) are added to the soap solution to decompose the soap into free fatty acids and glycerol. A few pieces of pumice stone (prepared by throwing the pieces at white heat into distilled water and keeping them under water until used) are added, the flask connected with a glass con- 
denser, heated slowly till boiling begins, and the contents then distilled at such a rate as will bring $110 \mathrm{cc}$. of the distillate over in as nearly thirty minutes as possible.

The distillate is mixed thoroughly and filtered through a dry filter; 100 cc. of the filtrate are poured into a 250 cc. beaker and titrated with a deci-normal barium-hydrate solution, half a cubic centimeter of phenolphtalein solution being used as an indicator. A blank test is made in the same manner as described, and the amount of alkali solution used deducted from the results obtained with the samples analyzed. The number of cubic centimeters of barium-hydrate solution used is increased by one-tenth, and the so-called Reichert or Reichert-Meissl number thus obtained.

The Reichert number for pure butter fat will ordinarily come above $24 \mathrm{cc}$. and may go over $30 \mathrm{cc}$; butter fat from stripper cows will have a low Reichert num. ber. Pure oleomargarine will have a Reichert number of 1 to 2 cc.; and mixtures of artificial and natural butter will give intermediate numbers.

Tests for the Detection of Oleomargarine or RenoVATED BUTTER.

283. The boiling test. ${ }^{1}$ A piece of butter of the size of a small chestnut is melted in an ordinary tablespoon (or a small tin dish) at a low heat, stirring with a splinter of wood. The heat is increased until as brisk a boil

1 Patrick, Household tests for the detection of oleomargarine and renovated butter, Farmer's Bulletin, No. 131. For detection and examination of renovated or "process" butter, see also Cochran, Journ. Frankl. Inst., 1899, p. 94 ; Analyst, 1899, p. SS. 
as possible, and after boiling has begun, the melted mass is stirred thoroughly two or three times, always shortly before boiling ceases. Oleomargarine and renovated butter will boil noisily, sputtering like a mixture of grease and water when boiled, and will produce but little or no foam. Renovated butter produces usually a very small amount of foam, while genuine butter boils with less noise and produces an abundance of foam.

284. The Waterhouse test for distinguishing oleomargarine and renovated butter. ${ }^{1}$ Half fill a 100 ce. beaker with sweet skim milk (or distilled water), heat nearly to boiling and add 5 to 10 grams of butter or oleomargarine. Stir with a small wooden stick of about the size of a match until the fat is melted; the beaker is then placed in ice water, and the milk (or water) stirred until the temperature falls sufficiently for the fat to congeal. If oleomargarine, the fat can now be easily collected into one lump by means of the stick, while if genuine or renovated butter, the fat will granulate and can not be so collected. ${ }^{2}$

\section{D.-Cheese.}

For method of sampling, see par. 104.

285. a. Water. Five grams of cheese cut into very thin slices are weighed into a small porcelain dish filled about one-third full with freshly-ignited stringy asbestos; the dish is placed in a water oven and heated for ten hours. The loss in weight is taken to represent water.

1 Farmers' Bulletin No. 131, p. 7 .

2 For tests for artificial coloring matter in oleomargarine, see Circ. 629 , Com. of Internal Rev., Treasury Dept. 
(Śee also Dean's method for determining water in butter, curd and cheese, par. 276.

286. b. Fat. About 5 grams of cheese are ground finely in a small porcelain mortar with about twice its weight of anhydrous copper sulfate, until the mixture is of a uniform light blue color and the cheese evenly distributed throughout the mass. The mixture is transferred to a glass tube of the kind used in butter analysis (263), only a larger size; a little copper sulfate is placed at the bottom of the tube, then the mixture containing the cheese, and on top of it a little extracted absorbent cotton or ignited stringy asbestos; the tube is placed in an extraction apparatus and extracted with anhydrous ether for fifteen hours. The ether is then distilled off, the flasks dried in a water oven at $100^{\circ} \mathrm{C}$. to constant weight, cooled and weighed. The method is apt to give too low results and, therefore, not to be preferred to the Babcock test for cheese (105).

287. c. Casein (total nitrogen $\times 6.25$ ). About 2 grams of cheese are weighed out on a watch glass and transferred to a Jena nitrogen flask, and the nitrogen in the sample determined according to the Kjeldahl method (253); the percentage of nitrogen multiplied by 6.25 gives the total nitrogenous components of the cheese.

288. d. Ash. The residue from the water determination is taken for the ash; it is preferably set fire to, in the same manner as explained under determination of ash in butter (270), before it is placed in the muffle oven and incinerated. The increase in the weight above that of the empty dish+asbestcs, gives the amount of ash in the sample weighed out. 
289. e. Other constituents. The sum of the percentages of water, fat, casein and ash, subtracted from 100, will give the per cent. of other constituents, organic acids, milk sugar, etc., in the cheese.

\section{Detection of Oleomargarine Cheese ("Filled" \\ Chkiese.)}

290. About 25 grams of finely-divided cheese are extracted with ether in a Caldwell extractor or a paper extraction cartridge; the ether is distilled off, and the fat dried in the water oven until there is no further loss in weight. 5.75 cc. of the clear fat are then measured into'a 250 cc. saponification flask and treated according to the Reichert-Wollny method, as already explained under Detection of Artificial Butter (282). ${ }^{1}$

Tesing for Adulteration of Milk and Cream.

29I. Use of the refractometer. The immersion refractometer furnishes a delicate apparatus for the detection of watered milk. ${ }^{2} 100$ cc. of milk and 2 cc. of $25 \%$ acetic acid are heated for twenty minutes at $70^{\circ} \mathrm{C}$. This is then placed on ice for ten minutes and filtered. The refractometer reading of the clear filtrate is then taken at $20^{\circ} \mathrm{C}$. If this reading is above 40 the milk is not watered, while figures below 40 show adulteration by watering.

291a. The nitric acid test may prove useful as cor. roborating evidence that a sample of milk has been watered (126). Normal fresh milk does not contair

${ }^{1}$ See Arb. Kais. Ges.-Amt., 14, 506-598.

${ }^{2}$ Leach, Food Apalysis, 2nd ed., p. 168. 
nitrates, while common well-water, particularly on farms where precautions to guard against contamination of the water supply have not been taken, in general contains appreciable amounts of nitrates, nitrites and ammonia compounds, and watered milk will, therefore, in such cases also contain nitrates. ${ }^{1}$ The method for detection of small amounts of nitrates in milk, as given by Richmond ${ }^{2}$ is as follows: Place a small quantity of diphenylamin at the bottom of a porcelain dish, and add to it about 1 cc. of pure $\mathrm{H}_{2} \mathrm{SO}_{1}$ (conc.) ; allow a few drops of the milk serum (obtained by adding a little acetic acid to the milk and warming) to flow down the sides of the dish and over the surface of the acid. If a blue color develops in the course of ten minutes, though it may be faint, it shows the presence of nitrates ; after ten minutes a reddish-brown color is always developed from the action of the acid on the serum. 'There should be no difficulty in detecting an addition of 10 per cent. of water to the milk by this test, if the water added contained 5 parts of nitric acid, or more, per 100,000 .

The following test for nitric acid is proposed by McKay and Bouska: About 5 cc. of milk is placed in a test tube. Some Kaniss' reagent (about 1 part formaldehyd in 500 cc. C. $\mathrm{P} . \mathrm{H}_{2} \mathrm{SO}_{4}$ ) is poured down the side of the tube so it will form a layer under the milk. If nitrates or nitrites are present, a violet ring will form at the place of contact. This is Hehner's test for formaldehyd reversed, see (304).

${ }^{1}$ Uffelmann, Deutsche Vierteljahresschr. f. öff. Ges.-pfl. 15, p. 663.

2 The Analyst, 1893 , p. 272. 
292. Besides by the methods given in the preceding (pp.121-127), watering or skimming of milk may be detected by determining the specific gravity of $a$, the skim milk, $b$, the milk serum, and $c$, the whey.

a. Specific gravity of skim milk. The milk is set in a flat porcelain or glass dish for $12-24$ hours in a cold room; the layer of cream formed is then skimmed off, and the sp. gr. of the skim milk determined-at $60^{\circ} \mathrm{F}$. Skim milk has a sp. gr. of .002 to .0035 (2 to 3.5 lactometer degrees) above that of the corresponding whole milk; a smaller difference than this indicates that the milk was skimmed. If both skimming and watering had been practiced, the difference given above might be obtained, but the analysis of the milk would in such case easily disclose the adulteration.

b. Specific gravity of the milk serum. To 100 cc. milk 2 ce. of 20 per cent.-acetic acid are added, and the mixture heated in a covered beaker or closed flask for 5-10 min. on a water-bath at $55-65^{\circ} \mathrm{C}$. After cooling, the milk serum is filtered off and its sp. gr. determined at $60^{\circ} \mathrm{F}$. In case of pure milks, the sp. gr. of the milk serum (at $60^{\circ}$ ) will come above 1.0270. Serum from normal milk contains 6.3 to 7.5 per cent. solids and .22 to .28 per cent. fat; by the addition of 10 per cent. of water, the solids in the serum are lowered .3 to .5 per cent., and the sp. gr., $.0005 .^{1}$

c. Specific gravity of whey. 500 ce. of milk are warmed in water of $40-50^{\circ}$ C. until its temperature is $35^{\circ} \mathrm{C}$; ; one-half cc. of rennet extract (12-15 drops) is added, and the milk stirred thoroughly. After allowing the eurd to solidify for 10 minutes, it is cut and the whey filtered off through several layers of cheese cloth. The whey must be clear; it is cooled to $60^{\circ} \mathrm{F}$. and its sp. gr. determined. The sp. gr. of whey from normal milk obtained in the manner given will range between 1.027 and 1.031. A sp. gr. of 1.026 or below indicates watering. An addition of 4 per cent. of water lowers the sp. gr. of the whey about 1 lactometer degree. ${ }^{2}$

${ }^{1}$ König, Menschl. Nahrungsmittel, II, p. 276.

2 Stats. Unters. landw. wicht. Stoffe, p. 88. 
293. Detection of coloring matter. Milk which has been watered or skimmed, or both, is sometimes further adulterated by unscrupulous milk dealers by an addition of a small quantity of cheese color; this will mix thoroughly with the milk, and, if added judiciously, will impart a rich cream color to it. The presence of foreign coloring matter in milk is easily shown by shaking 10 cc. of the milk with an equal quantity of ether; on standing, a clear ether solution will rise to the surface; if artificial coloring matter has been added to the milk, the solution will be yellow colored, the intensity of the color indicating the quantity addded; natural fresh milk will give a colorless ether solution.

A method gived by Wallace ${ }^{1}$ is claimed to detect one part of coloring matter in 100,000 of milk.

Inorganic coloring matter like chromates and bi-chromates have, although fortunately rarely, been used to impart a rich color to adulterated milk or poor cream. Chromates may be detected by the reddish yellow color produced when a little 2 per cent.-silver nitrate solution is added to a few cubic centimeters of the milk.

294. Detection of pasteurized milk or cream. Prof. Storch, of Copenhagen, Denmark, ${ }^{2}$ in 1898, published a simple method for ascertaining whether milk, cream, ol other dairy products have been heated to at least $176^{\circ} \mathrm{F} .\left(80^{\circ} \mathrm{C}.\right)$. The test is made as follows: A teaspoonful of the milk is poured into a test tube, and 1 drop of a weak solution of peroxid of hydrogen (2 per cent.) and 2 drops of a paraphenylenediamin-solu-

1 N. J. Dairy Commissioner, report 1896, p. 36.

240 th report, Copenhagen experiment station. 
tion ( 2 per cent.) are added. The mixture is then shaken; if a dark violet color appears at once, the milk has not been heated, or at any rate not beyond $176^{\circ} \mathrm{F}$. If a sample of butter is to be examined, 25 grams are placed in a small beaker and melted by being placed in water of $60^{\circ} \mathrm{C}$. The clear butter fat is poured off, and the remaining liquid is diluted with an equal volume of water. The mixture thus obtained is examined as in case of milk.

Guaiacum tincture has also been recommended for the detection of pasteurized cream or milk; this solution is easily obtained, keeps well, and is convenient to use (McKay).

295. Boiled milk. The preceding tests will serve to distinguish between raw and boiled milk, and also to ascertain if milk has been adulterated with diluted condensed milk. To what extent such an adulteration can be practiced without being detected by this or similar tests, has not been determined, but if a control test be made at the same time with a sample of milk of known purity, a small admixture of boiled (or diluted condensed) milk can doubtless be detected. ${ }^{1}$

296. Gelatine in cream. This method of adulteration is sometimes practiced in the city cream trade, to impart stiffness and an appearance of richness to the cream. To detect the gelatine, a quantity of the suspected cream is mixed with warm water, and acetic acid is added to precipitate the casein and fat ( 1.5 cc. of 10 per cent.-acetic acid per 10 cc. of cream is sufficient).

1 See also Siats, Unters. landw. wicht. Stoffe, p. 60, and MolkereiZtg. (Hildesheim), 1899, p. 677. 
The precipitate is filtered off, and a few drops of a strong tannin solution are added to the clear filtrate. Pure cream will give a slight precipitate, while in the presence of gelatine a copious precipitate will come down.

The picric-acid method has also been proposed for the detection of small quantities of gelatine in cream. ${ }^{1}$

297. Starch in cream. Starch is mentioned in the dairy literature as an adulterant of milk and cream. It is doubtful, however, if it is ever used for this purpose at the present time. In the case of ice-cream, on the other hand, a small quantity of corn starch is often added to thicken the milk used. It may in such a case be readily detected by means of the iodin reaction. A solution of iodin will produce a deep blue color in the presence of starch; a small amount of iodin is taken up by the cream before the blue coloration appears.

298. Macroscopic impurities (particles of hay, litter, woolen or cotton fibres, dung, etc.). These impurities may be separated by repeated dilution of the milk with pure distilled water, leaving the mixture undisturbed for a couple of hours each time before the liquid is syphoned off. When the milk has been entirely removed in this manner, the residue is filtered off, dried and weighed. A quart of milk or cream should not give any visible sediment on standing for several hours.

A simple and striking method of showing dirt in milk has been suggested by Gerber. About a pint of milk is poured into an inverted bottomless long-necked

1 'The Analyst, 1897 , p. 320. 
bottle, over the mouth of which a piece of cotton is placed. The milk will filter through, leaving the dirt on the cotton, which is then removed and ean be shown to the producer of the milk. ${ }^{1}$

A modification of the apparatus used has recently been described in a publication of the Wisconsin experiment station."

\section{Detection of Preservatives in Dairy Products.}

299. a. Boracic acid (borax, borates, preservaline, etc.). 100 cc. of milk are made alkaline with a soda or potash solution, and then evaporated to dryness and incinerated. The ash is dissolved in water to which a little hydrochloric acid has been added, and the solution filtered. A strip of turmeric paper moistened with the filtrate will be colored reddish brown when dried at $100^{\circ} \mathrm{C}$. on a watch glass, if boracic acid is present.

If a little alcohol is poured over the ash to which concentrated sulfuric acid has been added, and fire is set to the alcohol; this will burn with a yellowish green tint, especially noticeable if the ash is stirred with a glass rod and when the flame is about to go out.

300. The following modification of the first test given is said to show the presence of only a thousandth of a grain of borax in a drop of milk (about .15 per cent.) : ${ }^{3}$

Place in a porcelain dish one drop of milk with two drops of strong hydrochloric acid and two drops of saturated turmeric tincture; dry this on the water bath, cool and add a drop of ammonia by means of a glass rod. A slaty blue color changing to green is produced if borax is present."

${ }^{1}$ Hoard's Dairyman, Nov, 29, 1907.

${ }^{2}$ Bull. 195.

${ }^{3}$ N. J. Dairy Commissioner, report, 1896 p. 36.

${ }^{4}$ Sce also par. 151. 
30I. b. Bi-carbonate of soda. $100 \mathrm{cc}$. of milk to which a few drops of alcohol are added, are evaporated and carefully incinerated; the proportion of carbonic acid in the ash as compared with that of milk of known purity is determined. If an apparatus for the determination of carbonic acid is available, like the Scheibler apparatus, etc., the per cent. of carbonic acid per gram of ash (and quart of milk) can be easily ascertained. Normal milk ash contains only a small amount of carbonic acid (less than 2 per cent.), presumably formed from the citric acid of the milk in the process of incineration.

The following qualitative test is easily made: To 10 cc. of milk add $10 \mathrm{cc}$. of alcohol and a little of a one per cent. rosolic-acid solution. Pure milk will give a brownish yellow color; milk to which soda has been added, a rose red color. A control experiment with milk of known purity should be made.

302. c. Fluorids. 100 cc. of milk are evaporated in a platinum or lead crucible, and incinerated; the ash is made strongly acid with concentrated sulfuric acid. If fluorids are present hydrofluoric acid will be generated on gentle heating and will be apparent from its etching a watch glass placed over the crucible. ${ }^{1}$

303. d. Salicylic acid (salicylates, etc.). 20 cc. of milk are acidulated with sulfuric acid and shaken with ether; the ether solution is evaporated, and the residue treated with alcohol and a little iron-chlorid solution;

\footnotetext{
1 Chromates in dairy products may be readily determined by the use of a silver-nitrate solution, see Molkerei-Ztg. (Berlin) 1899, p. 603.
} 
a deep violet color will be obtained in the presence of salicylic acid.

304. e. Formaldehyde (a forty-per cent. solution in water).

The following method by Hehner is stated to show the presence of one part of formaldehyde in 200,000 parts of milk: the milk is diluted with an equal volume of water, and strong $\mathrm{H}_{2} \mathrm{SO}_{4}$ (sp. gr. 1.82-1.84) is added. A violet ring is formed at the junction of the two liquids if formaldehyde is present; if not, a slight greenish tinge will be seen. The violet color is not obtained with milk containing over .05 per cent. formaldehyde. ${ }^{1}$

The same color reaction is obtained in the Babcock test and is easily recognized by persons familiar with milk testing when their attention has once been called to the characteristic color.

An adulteration of milk with formaldehyde may be readily detected by the following method, which will show the presence of only a trace of formaldehyde in the milk. 5 cc. of milk is measured into a white porcelain dish, and a similar quantity of water added. 10 cc. of $\mathrm{HCl}$ containing a trace of $\mathrm{Fe}_{2} \mathrm{Cl}_{6}$ is added, and the mixture is heated very slowly. If formaldehyde is present, a violet color will be formed.

${ }^{1}$ Chem. News, 1896, No. 71 ; Milchzeitung, 1896, 491; 1897, 40, 667 ; The Analyst, 1895, 152, 154, 157; 1896, 285. 


\section{GOVERNMENT STANDARDS OF PURITY FOR MILK AND ITS PRODUCTS. ${ }^{1}$}

a. MILKS.

1. Milk is the fresh, clean, lacteal secretion obtained by the complete milking of one or more healthy cows, properly fed and kept, excluding that obtained within fifteen days before and ten days after calving, and contains not less than eight and onehalf (8.5) per cent. of solids not fat, and not less than threo and one-quarter (3.25) per cent. of milk fat.

2. Blendid milk is milk modified in its composition so as to have a definite and stated percentage of one or more of its con. stituents.

3. Skim milk is milk from which a part or all of the cream has becn removed and contains not less than nine and one-quarter (9.25) per cent. of milk solids.

4. Pasteurized milk is milk that has been heated below boiling but sufficiently to kill most of the active organisms present and immediately cooled to $50^{\circ} \mathrm{Fehr}$. or lower.

5. Sterilized milk is milk that has been heated at the tem. perature of boiling water or higher for a length of time suffi. cient to kill all organisms present.

6. Condensed mllk, evaporated milk, is milk from which a considerable portion of water has been evaporated and contains not less than twenty-eight (28) per cent. of milk solids, of which not less than twenty-seven and five-tenths (27.5) per cent. is milk fat.

7. Sweetened condensed milk is milk from which a considcrable portion of water has been evaporated and to which sugar (sucrose) has been added, and contains not less than twentyeight (28) per cent of milk solids, of which not less than twenty-seven and five-tenths (27.5) per cent. is milk fat.

\footnotetext{
${ }^{1}$ Circular No. 19, Office of the Secretary, U. S. Dept. of Agriculture, June 26, 1906 .
} 
8. Condensed skim milk is skim milk from which a considerable portion of water has been evaporated.

9. Buttermilk is the product that remains when butter is removed from milk or cream in the process of churning.

10. Goat's milk, ewe's milk, etc., are the fresh, clean, lacteal secretions, free from colostrum, obtained by the complete milking of healthy animals other than cows, properly fed and kept, and conform in name to the species of animal from which they are obtained.

\section{1). CREAM.}

1. Cream is that portion of milk, rich in milk fat, which rises to the surface of milk on standing, or is separated from it by centrifugal force, is fresh and clean and contains not less than eighteen (18) per cent. of milk fat.

2. Evaporated cream, clotted cream, is cream from which a considerable portion of water has been evaporated.

c. MILK FAT OR BUTTER FAT.

1. Milk fat, butter fat, is the fat of milk and has the Reichert-Meissl number not less than twenty-four (24) and a specific gravity of not less than $0.905\left(\frac{40^{\circ} \mathrm{O} .}{40^{\circ} \mathrm{O} .}\right)$

d. BUTTER.

1. Butter is the clean, non-rancid product made by gathering in any manner the fat of fresh or ripened milk or cream into a mass, which also contains a small portion of the other milk constituents, with or without salt, and contains not less than eighty-two and fivertenths (82.5) per cent. of milk fat. By acts of Congress approved August 2, 1886, and May 9, 1902, butter may also contain added coloring matter.

2. Renovated butter, process butter, is the product made by melting butter and reworking, without the addition or use of chemicals or any substances except milk, cream, or salt, and contains not more than sixteen (16) per cent. of water and at least eighty-two and five-tenths (82.5) per cent. of milk fat. 
e. CHEESE.

1. Cheese is the sound, solid, and ripened product made from milk or cream by coagulating the casein thereof with rennet or lactic acid, with or without the addition of ripening ferments and seasoning, and contains, in the water-free substance, not less than fifty (50) per cent. of milk fat. By act of Congress, approved June 6, 1£96, cheese may also contain added coloring matter.

2. Skim milk cheese is the sound, solid, and ripened product, made from skim milk by coagulating the casein thereof with rennet or lactic acid, with or without the addition of ripening ferments and seasoning.

3. Goat's milk cheese, ewe's milk cheese, etc., are the sound, ripened products made from the milks of the animals specified, by coagulating the casein thereof with rennet or lactic acid, with or without the addition of ripening ferments and seasoning.

\section{f. ICE CREAMS.}

1. Ice cream is a frozen product made from cream and sugar, with or without a natural flavoring, and contains not less than fourteen (14) per cent. of milk fat.

2. Fruit ice cream is a frozen product made from cream, sugar, and sound, clean, mature fruits, and contains not less than twelve (12) per cent. of milk fat.

3. Nut ice cream is a frozen product made from cream, sugar, and sound, non-rancid nuts, and contains not less than twelve (12), per cent. of milk fat.

\section{g. MISCELLANEOUS MILK PRODUCTS.}

1. Whey is the product remaining after the removal of fat and casein from milk in the process of cheese-making.

2. Kumiss is the product made by the alcoholic fermentation of mare's or cow's milk. 
STANDARDS FOR BABCOCK GLASSWARE. (Adopted by the Association of Official Agricultural Chemists of North America.)

SEc. 1. The unit of graduation for all Babcock glassware shall be the true cubic centimeter (.998877 gram of water at $4^{\circ}$ C.).

(a) With bottles, the capacity of each per cent. on the scale shall be two-tenths $(0.20)$ cubic centimeter.

(b) With pipettes and acid measures the delivery shall be the intent of the graduation and the graduation shall be read with the bottom of the meniscus in line with the mark.

SEc. 2. The official method for testing bottles shall be calibration with mercury (13.5471 grams of clean, dry mercury at $20^{\circ}$ C., carefully weighed on analytical balances, to be equal to 5 per cent. on the Babcock scale), the bottles being previously filled to zero with mereury.

SEc. 3. Optional methods.-The mercury and cork, alcohol and burette, and alcohol and brass plunger methods may be employed for the rapid-testing of Babcock bottles, but the accuracy of all questionable bottles shall be determined by the official method.

SEC. 4. The official method for testing pipettes and acid measures shall be calibration by measuring in a burette the quantity of water (at $20^{\circ}$ C.) delivered.

SEc. 5. The limits of error.-(a) For Babcock bottles shall be the smallest graduation on the scale, but in no case shall it exceed five-tenths (0.50) per cent., or for skim milk bottles onehundredth (0.01) per cent.

(b) For full-quantity pipettes, it shall not exceed one-tenth (0.10) cubic centimeter, and for fractional pipettes, five-hundredths (0.05) cubic centimeter.

(c) For acid measures it shall not exceed two-tenths $(0.20)$ cubic centimeter. 



\section{APPENDIX.}

Table 1. Composition of milk and its products.

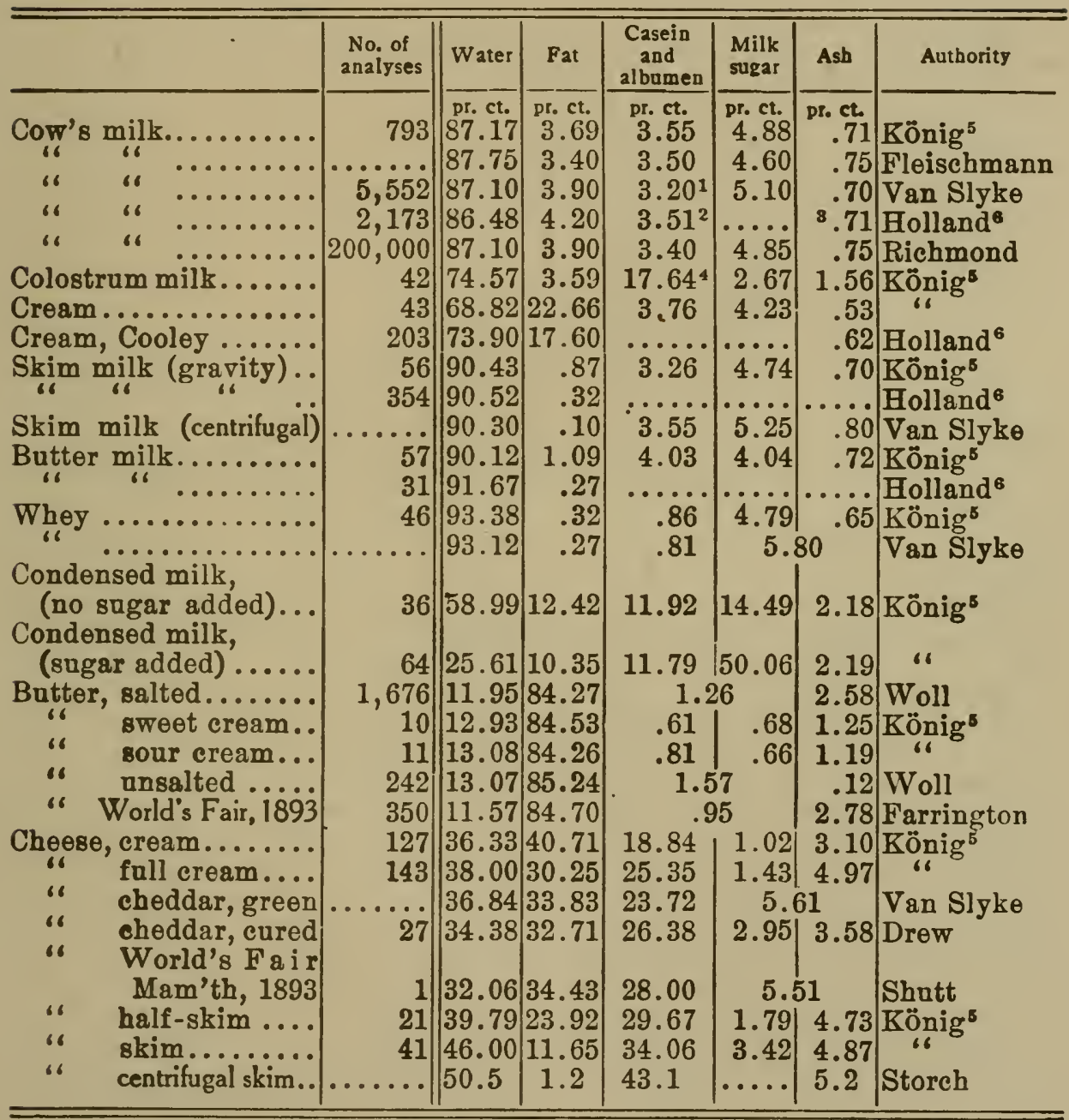

1.70 per cent. albumen.

Forty-two analyses.

8 Elght analyses.
413.60 per cent. albumen.

- Mostly European samples.

6 Massachusetts' samples. 


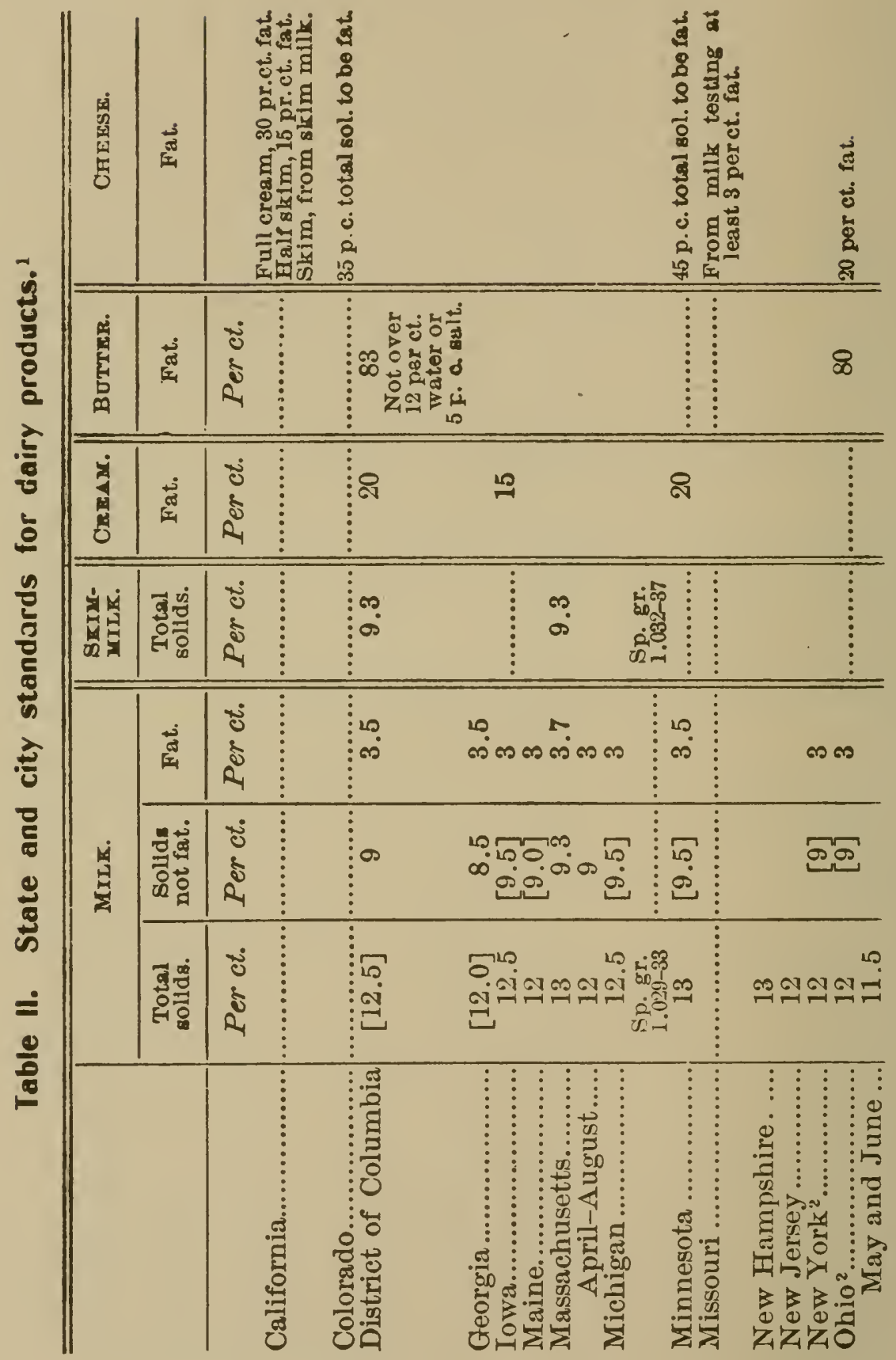




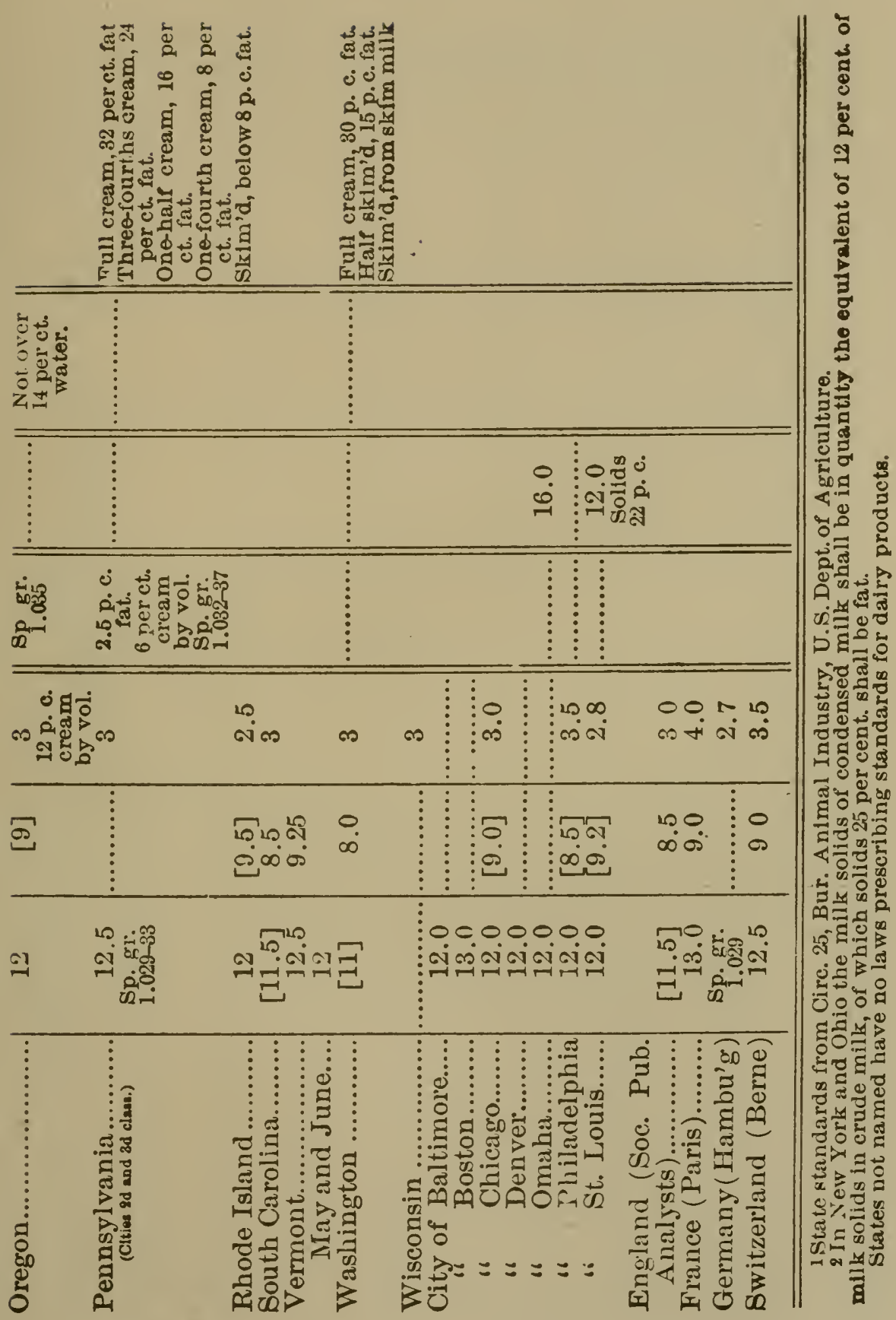


Table III. Quevenne lactometer degrees corresponding to N. Y. Board of Health degrees. (See par. 114)

\begin{tabular}{c|c||c|c||c|c}
\hline $\begin{array}{c}\text { Bd. of Health } \\
\text { degrees. }\end{array}$ & $\begin{array}{c}\text { Quevenne } \\
\text { scale. }\end{array}$ & $\begin{array}{c}\text { Bd. of Health } \\
\text { degrees. }\end{array}$ & $\begin{array}{c}\text { Quovenne } \\
\text { scale. }\end{array}$ & $\begin{array}{c}\text { Bd. of Health } \\
\text { degrees. }\end{array}$ & $\begin{array}{c}\text { Quevenne } \\
\text { scale. }\end{array}$ \\
\hline 60 & 17.4 & 81 & 23.5 & 101 & 29.3 \\
\hline 61 & 17.7 & 82 & 23.8 & 102 & 29.6 \\
62 & 18.0 & 83 & 24.1 & 103 & 29.9 \\
63 & 18.3 & 84 & 24.4 & 104 & 30.2 \\
64 & 18.6 & 85 & 24.6 & 105 & 30.5 \\
65 & 18.8 & 86 & 24.9 & 106 & 30.7 \\
66 & 19.1 & 87 & 25.2 & 107 & 31.0 \\
67 & 19.4 & 88 & 25.5 & 108 & 31.3 \\
68 & 19.7 & 89 & 25.8 & 109 & 31.6 \\
69 & 20.0 & 90 & 26.1 & 110 & 31.9 \\
70 & 20.3 & 91 & 26.4 & 111 & 32.2 \\
71 & 20.6 & 92 & 26.7 & 112 & 32.5 \\
72 & 20.9 & 93 & 27.0 & 113 & 32.8 \\
73 & 21.2 & 94 & 27.3 & 114 & 33.1 \\
74 & 21.5 & 95 & 27.6 & 115 & 33.4 \\
75 & 21.7 & 96 & 27.8 & 116 & 33.6 \\
76 & 22.0 & 97 & 28.1 & 117 & 33.9 \\
77 & 22.3 & 98 & 28.4 & 118 & 34.2 \\
78 & 22.6 & 99 & 28.7 & 119 & 34.5 \\
79 & 22.9 & 100 & 29.0 & 120 & 34.8 \\
80 & 23.2 & & & & \\
\hline
\end{tabular}

Table IV. Value of $\frac{100 s-100}{\mathrm{~s}}$ for sp. gr. from 1.019 to 1.0369 .

\begin{tabular}{c|c|c|c|c|c|c|c|c|c|c}
\hline \hline Sp. gr. (s) $=$ & 0.0000 & 0.0001 & 0.0002 & 0.0003 & 0.0004 & 0.0005 & 0.0006 & 0.0007 & 0.0008 & 0.0009 \\
\hline 1.019 & 1.864 & 1.874 & 1.884 & 1.894 & 1.903 & 1.913 & 1.922 & 1.932 & 1.941 & 1.951 \\
1.020 & 1.961 & 1.970 & 1.980 & 1.990 & 1.999 & 2.009 & 2.018 & 2.028 & 2.038 & 2.047 \\
1.021 & 2.057 & 2.066 & 2.076 & 2.086 & 2.095 & 2.105 & 2.114 & 2.124 & 2.133 & 2.143 \\
1.022 & 2.153 & 2.162 & 2.172 & 2.181 & 2.191 & 2.200 & 2.210 & 2.220 & 2.229 & 2.239 \\
1.023 & 2.249 & 2.258 & 2.267 & 2.277 & 2.286 & 2.296 & 2.306 & 2.315 & 2.325 & 2.334 \\
1.024 & 2.344 & 2.353 & 2.363 & 2.372 & 2.382 & 2.391 & 2.401 & 2.410 & 2.420 & 2.430 \\
1.025 & 2.439 & 2.449 & 2.458 & 2.468 & 2.477 & 2.487 & 2.496 & 2.506 & 2.515 & 2.525 \\
1.026 & 2.534 & 2.544 & 2.553 & 2.563 & 2.573 & 2.582 & 2.591 & 2.601 & 2.610 & 2.620 \\
1.027 & 2.629 & 2.638 & 2.648 & 2.657 & 2.667 & 2.676 & 2.686 & 2.695 & 2.705 & 2.714 \\
1.028 & 2.724 & 2.733 & 2.743 & 2.752 & 2.762 & 2.771 & 2.781 & 2.790 & 2.799 & 2.809 \\
1.029 & 2.818 & 2.823 & 2.837 & 2.847 & 2.856 & 2.865 & 2.875 & 2.884 & 2.893 & 2.903 \\
1.030 & 2.913 & 2.922 & 2.931 & 2.941 & 2.951 & 2.960 & 2.969 & 2.979 & 2.988 & 2.997 \\
1.031 & 3.007 & 3.016 & 3.026 & 3.035 & 3.044 & 3.054 & 3.063 & 3.072 & 3.082 & 3.091 \\
1.032 & 3.101 & 3.110 & 3.120 & 3.129 & 3.138 & 3.148 & 3.157 & 3.166 & 3.176 & 3.185 \\
1.033 & 3.195 & 3.204 & 3.213 & 3.223 & 3.232 & 3.241 & 3.251 & 3.260 & 3.269 & 3.279 \\
1.034 & 3.288 & 3.298 & 3.307 & 3.316 & 3.326 & 3.335 & 3.344 & 3.354 & 3.363 & 3.372 \\
1.035 & 3.382 & 3.391 & 3.400 & 3.410 & 3.419 & 3.428 & 3.438 & 3.447 & 3.456 & 3.466 \\
1.036 & 3.475 & 3.484 & 3.494 & 3.503 & 3.512 & 3.521 & 3.531 & 3.540 & 3.549 & 3.559 \\
\hline \hline
\end{tabular}


Appendix.

Table V. Correction-table for specific gravity of milk.

\begin{tabular}{|c|c|c|c|c|c|c|c|c|c|c|}
\hline \multirow{2}{*}{ 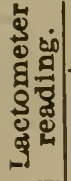 } & \multicolumn{10}{|c|}{ Temperature of milk (in degrees Fahrenheit). } \\
\hline & 51 & 52 & 53 & 54 & 55 & 56 & 57 & 58 & 59 & 60 \\
\hline 20 & 19.3 & 19.4 & 19.4 & 19.5 & 19.6 & 19.7 & 19.8 & 19.9 & 19.9 & 20.0 \\
\hline 21 & & 20.3 & 20.4 & 20.5 & 20.6 & 20.7 & 20.8 & 20.9 & & 21.0 \\
\hline 2.2 & 21.3 & 21.3 & 21.4 & 21.5 & 21.6 & 21.7 & 21.8 & 21.9 & 21.9 & 22.0 \\
\hline 23 & 22.3 & 22.3 & 22.4 & 22.5 & 22.6 & 22.7 & 22.8 & 22.8 & 22.9 & 23.0 \\
\hline 24 & 23.3 & 23.3 & 23.4 & 23.5 & 23.6 & 23.6 & 23.7 & 23.8 & 23.9 & 24.0 \\
\hline 25 & 24.2 & 24.3 & 24.4 & 24.5 & 24.6 & 24.6 & 24.7 & 24.8 & 24.9 & 25.0 \\
\hline 26 & 25.2 & 25.2 & 25.3 & 25.4 & 25.5 & 25.6 & 25.7 & 25.8 & & 26.0 \\
\hline 27 & 26.2 & 26.2 & 26.3 & 26.4 & 26.5 & 26.6 & 26.7 & 26.8 & 26.9 & 27.0 \\
\hline 28 & 27.1 & 27.2 & 27.3 & 27.4 & 27.5 & 27.6 & 27.7 & 27.8 & 27.9 & 28.0 \\
\hline 29 & 28.1 & 28.2 & 28.3 & 28.4 & 28.5 & 28.6 & 28.7 & 25.5 & 28.9 & 29.0 \\
\hline U & 29.1 & 29.1 & 29.2 & 29.3 & 29.4 & 29.6 & 29.7 & 29.8 & 29.9 & 30.0 \\
\hline 31 & 30 . & 30.1 & 30.2 & 30.3 & 30.4 & 30.5 & 30.6 & 30.8 & 30.9 & 31.0 \\
\hline 32 & 31.0 & 31. & 31. & 31.3 & 31.4 & 31.5 & 31.6 & 31.7 & 31.9 & 32.0 \\
\hline 33 & 31.9 & 32.0 & 32.1 & 32.3 & 32.4 & 32.5 & 32.6 & 32.7 & 32.9 & 33.0 \\
\hline 34 & 32.9 & 33.0 & 33.1 & 33.2 & 33.3 & 33.5 & $|33.6|$ & $33.7 \mid$ & 33.9 & 34.0 \\
\hline \multirow[t]{2}{*}{35} & 33.8 & 33.9 & 34.0 & 34.2 & 34.3 & 34.5 & 34.6 & 34.7 & 34.9 & 35.0 \\
\hline & 61 & 62 & 63 & 64 & 65 & 66 & 67 & 68 & 69 & 70 \\
\hline 20 & 20.1 & 20.2 & 20.2 & 20.3 & & 20.5 & 20.6 & 20.7 & 20.9 & 21.0 \\
\hline 21 & 21.1 & 21.2 & 21.3 & 21.4 & 21.5 & 21.6 & 21.7 & 21.8 & & 22.1 \\
\hline 22 & 22.1 & 22 . & 22 . & 23.4 & 22.5 & 22.6 & 22.7 & 22.8 & 23.0 & 23.1 \\
\hline 23 & 23. & 23. & 23 . & 23.4 & 23.5 & 23.6 & 23.7 & 23.8 & & 24.1 \\
\hline 24 & 24 . & 24 . & 24 & 24.4 & 24 & 24.6 & 24.7 & 24.9 & & 25.1 \\
\hline 25 & 25. & 25. & 25. & 25.4 & 25. & 25.6 & 25.7 & & & 26.1 \\
\hline 26 & 26.1 & 26 . & 26 & 26. & 26 & 26.7 & 26.8 & 27.0 & 27 & 27.2 \\
\hline 27 & 27.1 & 27. & 27. & 27.5 & 27. & 27.7 & & & & \\
\hline 28 & 28.1 & 28 . & & 28 & 28.6 & 28.7 & 28. & 29.0 & & 29.2 \\
\hline 29 & 29.1 & 29 . & 29 & 29 & 29.6 & 29 & & & & 30.3 \\
\hline 30 & 30.1 & 30 . & 30 & 30 & 30.7 & 30 . & 30.9 & 31. & & 31.3 \\
\hline 31 & 31. & 31. & 31 & 31. & 31.7 & 31.8 & & & 32.2 & 32.4 \\
\hline 32 & 32. & & 32 & 32. & 32.7 & 32.9 & 33 & & 33.3 & 33.4 \\
\hline 33 & 33. & 33. & 33 & 33. & 33.8 & 33.9 & & & 34.3 & 34.5 \\
\hline 34 & 34.2 & 34. & 34. & 34.6 & 34.8 & 34.9 & 35.0 & 35.2 & & 35 \\
\hline 35 & 35.2 & 35.3 & 35.5 & 35.6 & 35.8 & 35.9 & 36.1 & 36.2 & 36.4 & 36.5 \\
\hline
\end{tabular}

Directions. - Bring the temperature of the milk to within $10^{\circ}$ of $60 \%$ Take the reading of the lactometer and that of the temperature of the milk; find the former in the first vertical column of the table and the latter in the first horizontal row of figures; the figure where the horizontal and vertical columns meet is the corrected lactometer reading; e.g., observed, 31.0 at $67^{\circ} \mathrm{F}$.; corrected reading, 31.9. 
Table VI. Per cent. of solids not fat, corresponding to 0 to 6 per cent. of fat, and lactometer readings of 26 to 36. (See directions for use, par. 120)

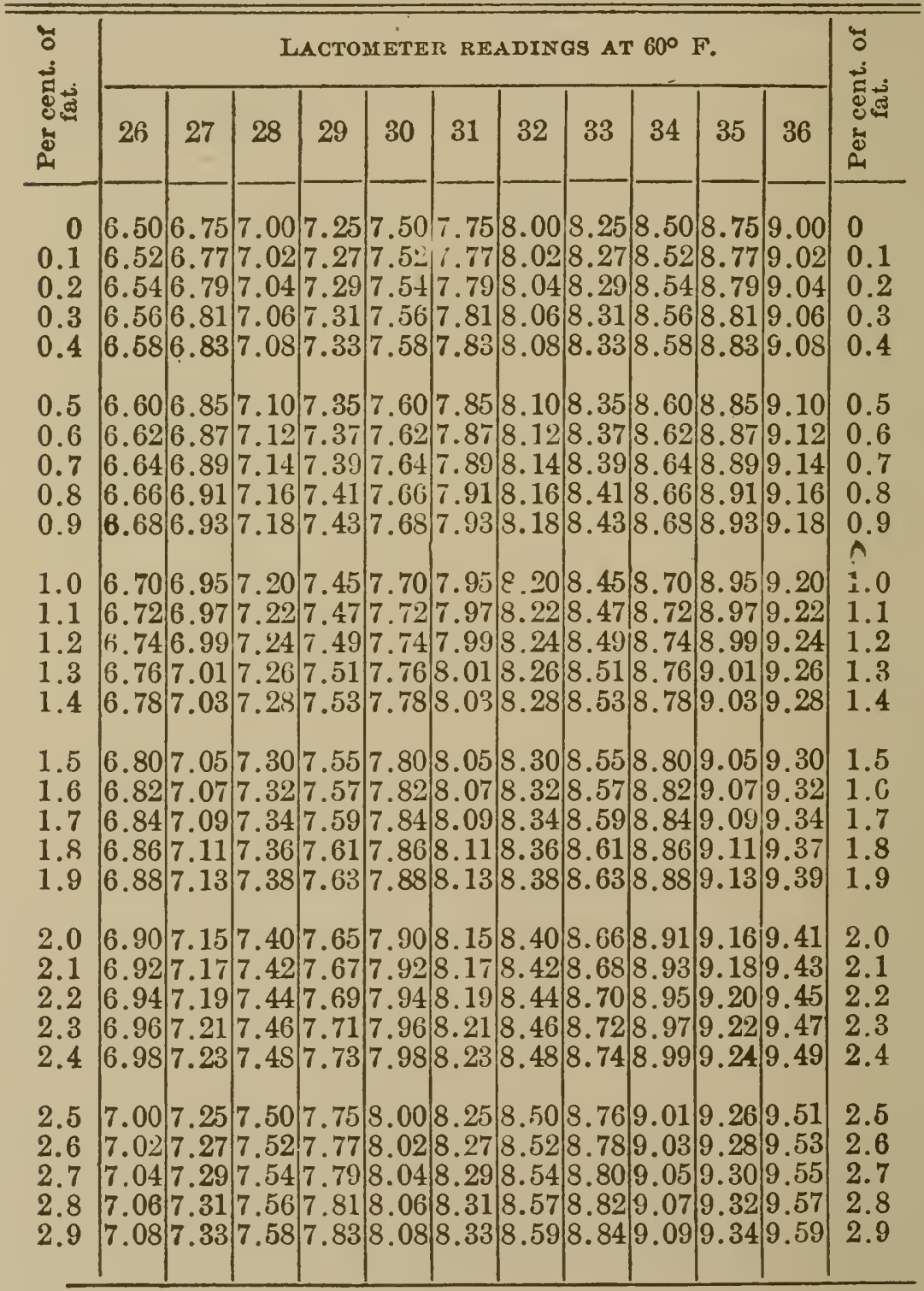


Iable VI. Per cent. of solids not fat (Continuea).

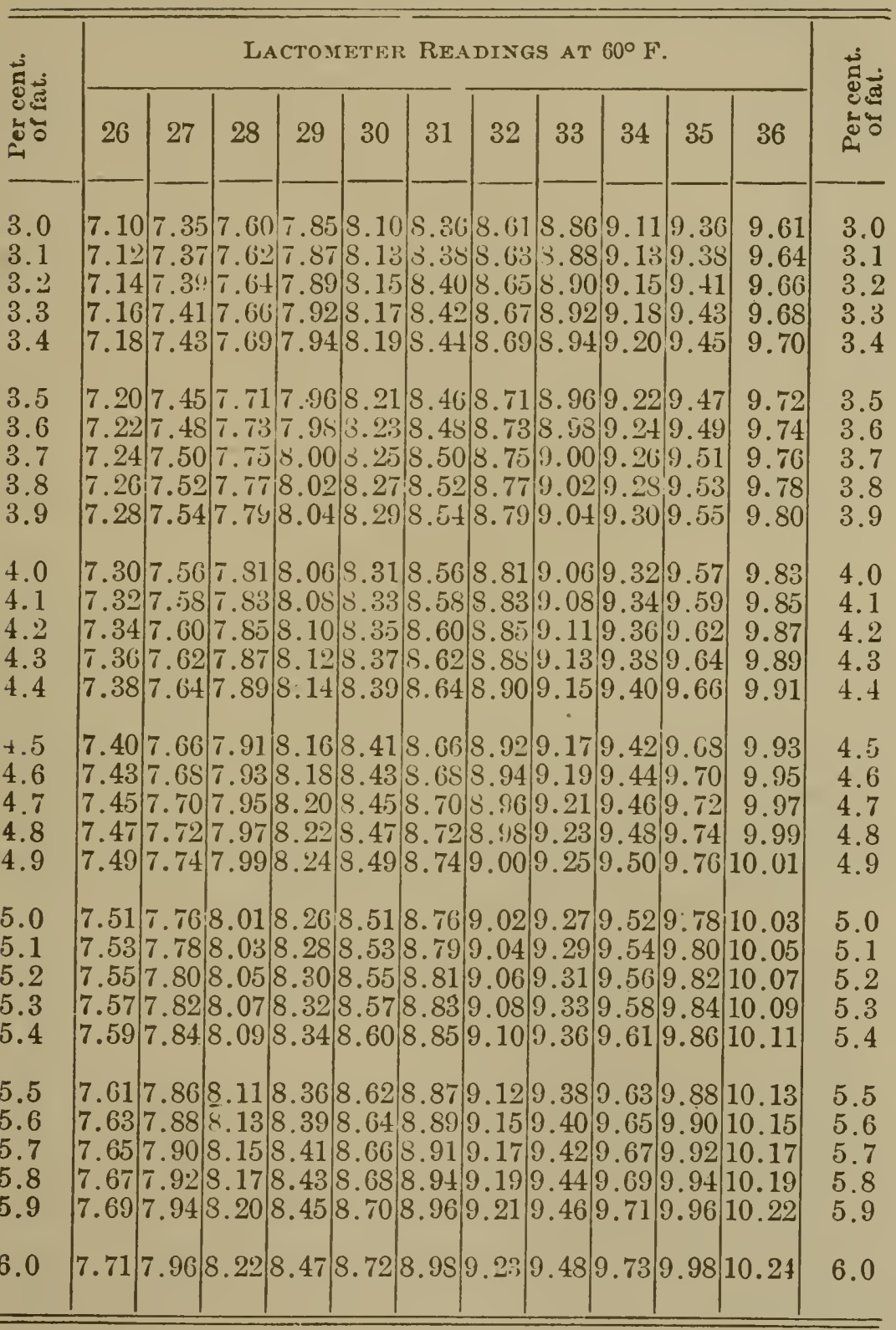




\section{Directions for Use of Tables VII, VIII, IX, and XI.}

TABLES VII, and VIII. Find the test of the milk in table VII or of cream in table VIII; the first or last horizontal row of figures, the amounts of fat in ten thousand, thousands, hundreds, tens, and units of pounds of milk are then given in this vertical column. By adding the corresponding figures for any given quantity of milk or of cream, the total quantity of butter fat contained therein is obtained.

Example: How many ponnds of fat is contained in 8925 lbs. of milk testing 3.65 per cent.? On p. 264, econd column the test 3.65 is found, and by going downward in this culumn we have:

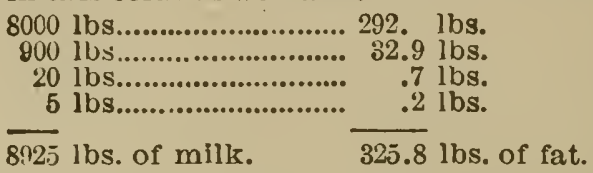

$8925 \mathrm{lbs}$. of milk testing 3.65 per cent., therefore, contains $395.8 \mathrm{lbs}$. of butter fat.

TABLC IX. The price per pound is giren in the outside vertical columns, and the weight of butter fat in the upper and lower horizontal row of figures. The corresponding tens of pounds are found by moving the decimal point one place to the left, the units, by moving it two, and the tenths of a pound, by moving it three places to the left. The use of thi table is, otherwise, as explained above.

Example: How much money is due for $325.8 \mathrm{lbs}$. of butter fat at $151 / 2$ cents per pound $?$ In the horizontal row of figures beginning with $151 / 8$ on p. 247, we find:

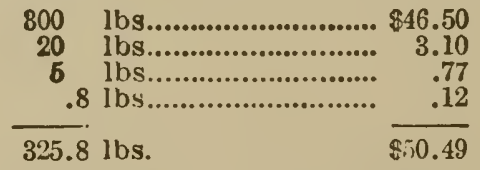

$825.8 \mathrm{lbs}$. of butter fat at $15 \frac{1}{2}$ cents per pound, therefore, is worth $\$ 50.49$.

TABLE XI. Find the test of milk in the upper or lower horizontal row of figures. The amounts of butter likely to be made from ten thousand, thousands, hundreds, tens, and units of pounds of milk are then given in this vertical column. The use of the table is, otherwise, as explained abnve in case of table VII.

Example: How much butter will $5845 \mathrm{lbs}$. of milk testing 3.8 per cent. be apt to make under good creamery conditions? In the column beaded 3.8, we find :

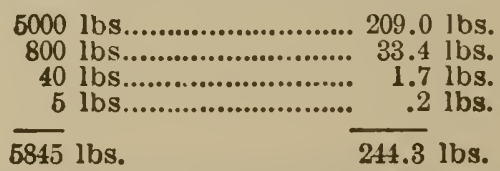

$5845 \mathrm{lbs}$. of milk testing 3.8 per cent. of fat will make about $244.3 \mathrm{lbs}$. of butter, under conditions similar to those explained in par. 220 . 
Appendix.

Table VII. Pounds of fat in I to 10,000 lbs. of milk, testing 3.0 to 5.35 per cent. (See directions for use, p. 265.)

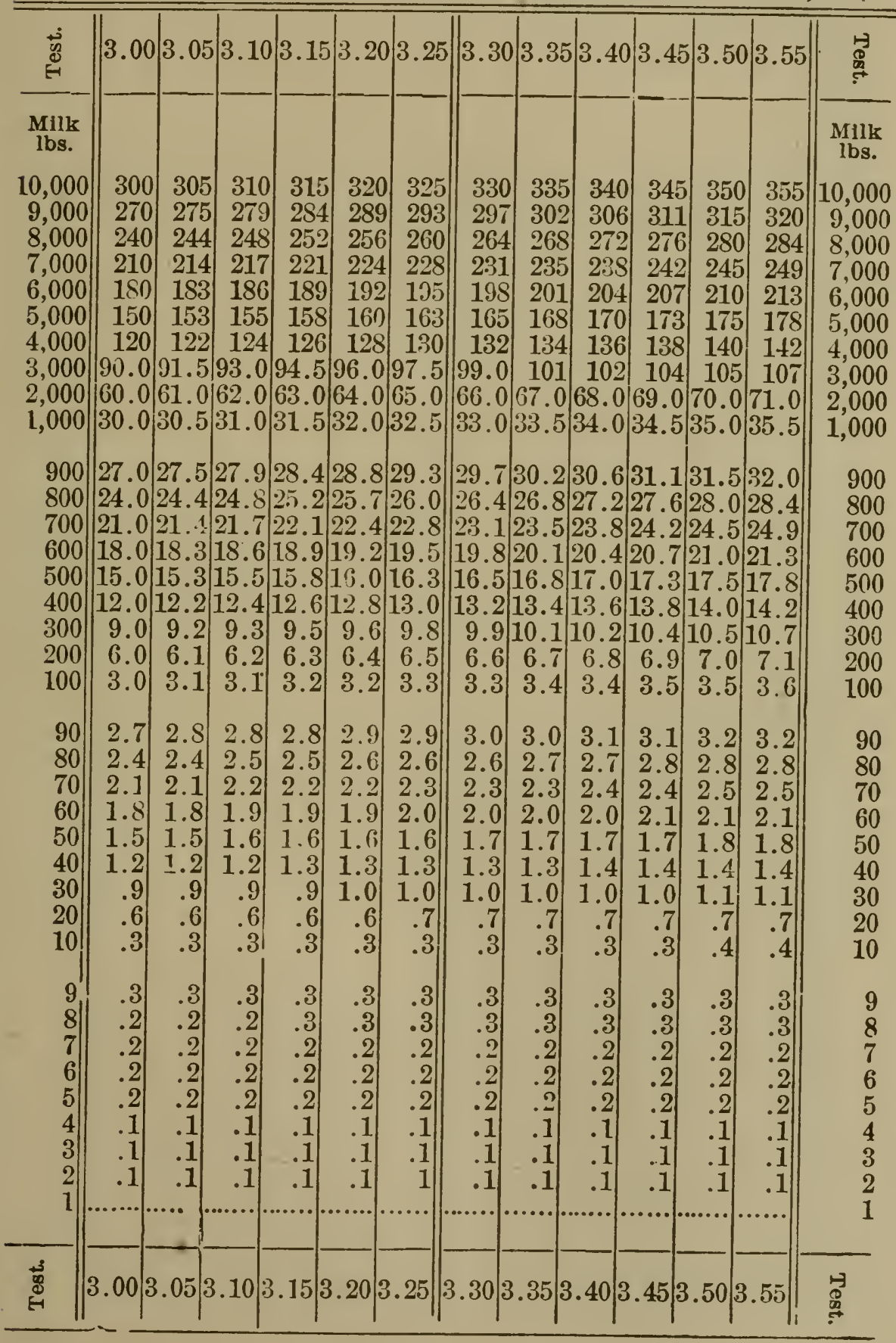


Table Vit. Pounds of fat in I to 10,000 Ibs. of milk (Continued).

\begin{tabular}{|c|c|c|c|c|c|c|c|c|c|c|c|c|c|}
\hline 莕 & 3.60 & 3.65 & 3.70 & 3.75 & 3.80 & 3.85 & 3.90 & 3.95 & 54.00 & 4.05 & 4.10 & 4.15 & 蜜 \\
\hline $\begin{array}{l}\text { Milk } \\
\text { lbs. }\end{array}$ & & & & & & & & & & & & & $\begin{array}{l}\text { Milk } \\
\text { lbs. }\end{array}$ \\
\hline 10,000 & 360 & 365 & 370 & 375 & 380 & 385 & 390 & 395 & 400 & 405 & 410 & 415 & 10,000 \\
\hline 9,000 & 324 & 329 & 333 & 338 & 342 & 347 & 351 & 356 & 360 & 365 & 369 & 374 & 9,000 \\
\hline 8,000 & 288 & 292 & 296 & 300 & 304 & 308 & 312 & 316 & 320 & 324 & 328 & 332 & 8,000 \\
\hline 7.000 & 252 & 256 & 259 & 263 & 266 & 270 & 273 & 277 & 280 & 284 & 287 & 291 & 7,000 \\
\hline 6,000 & 216 & 219 & 222 & 225 & 229 & 231 & 234 & 237 & 240 & 243 & 246 & 249 & 6,000 \\
\hline 5,000 & 180 & 183 & 185 & 188 & 190 & 193 & 195 & 198 & 200 & 203 & 205 & 208 & 5,000 \\
\hline 4,000 & 144 & 146 & 148 & 150 & 1.) 2 & 154 & 156 & 158 & 160 & 162 & 164 & 166 & 4,000 \\
\hline 3,000 & 108 & 110 & 111 & 113 & 114 & 116 & 117 & 119 & 120 & 122 & 123 & 125 & 3,000 \\
\hline 2,000 & 72.0 & 73.0 & 74.0 & 75.0 & 76.0 & 77.0 & 78.0 & 79.0 & 80.0 & 81.0 & 82.0 & 83.0 & 2,000 \\
\hline 1,000 & 36.0 & 36.5 & 37.0 & 37.5 & 38.0 & 38.5 & 39.0 & 39.5 & 40.0 & 40.5 & 41.0 & 41.5 & 1,000 \\
\hline 900 & 32.4 & 32.9 & 33.3 & 33.8 & 34.2 & 34.7 & $\mid 35.1$ & 35.6 & 36.0 & 36.5 & 36.9 & $37.4 \mid$ & 900 \\
\hline 800 & 28.8 & 29.2 & 29.6 & 30.0 & 30.4 & 30.8 & 31.2 & 31.6 & 32.0 & 32.4 & 32.8 & 33.2 & 800 \\
\hline 700 & 25.2 & 25.6 & 25.9 & 26.3 & 26.6 & 27.0 & 27.3 & 27.7 & 28.0 & 28.4 & 28.7 & 29.1 & 700 \\
\hline 600 & 21.6 & 21.9 & 22.2 & 22.5 & 22.8 & 23.1 & 23.4 & 23.7 & 24.0 & 24.3 & 24.6 & 24.9 & 600 \\
\hline 500 & 18.0 & 18.3 & 18.5 & 18.8 & 19.0 & 19.3 & 19.5 & 19.8 & 20.0 & 20.3 & 20.5 & 20.8 & 500 \\
\hline 400 & 14.4 & 14.6 & 14.8 & 15.0 & 15.2 & 15.4 & 15.6 & 15.8 & 16.0 & 16.2 & 16.4 & 16.6 & 400 \\
\hline 300 & 10.8 & 11.0 & 11.1 & 11.3 & 11.4 & 11.6 & 11.7 & 11.9 & 12.0 & 12.2 & 12.3 & 12.5 & 300 \\
\hline 200 & 7.2 & $\begin{array}{r}7.3 \\
\end{array}$ & 7.4 & 7.5 & 7.6 & 7.7 & 7.8 & 7.9 & 8.0 & 8.1 & 8.2 & 8.3 & 200 \\
\hline 100 & 3.6 & 3.7 & 3.7 & 3.8 & 3.8 & 3.9 & 3.9 & 4.0 & 4.0 & 4.1 & 4.1 & 4.2 & 100 \\
\hline 90 & 3.2 & 3.3 & 3.3 & 3.4 & 3.4 & 3.5 & 3.5 & 3.6 & 3.6 & 3.7 & 3.7 & 3.7 & 90 \\
\hline 80 & 2.9 & 2.9 & 3.0 & 3.0 & 3.0 & 3.1 & 3.1 & 3.2 & 3.2 & 3.2 & 3.3 & 3.3 & 80 \\
\hline 70 & 2.5 & 2.6 & 2.6 & 2.6 & 2.7 & 2.7 & 2.7 & 2.8 & 2.8 & 2.8 & 2.9 & 2.9 & 70 \\
\hline 60 & 2.2 & 2.2 & 2.2 & 2.3 & 2.3 & 2.3 & 2.3 & 2.4 & 2.4 & 2.4 & 2.5 & 2.5 & 60 \\
\hline 50 & 1.8 & 1.8 & 1.9 & 1.9 & 1.2 & 1.9 & 2.0 & 2.0 & 2.0 & 2.0 & 2.1 & 2.1 & 50 \\
\hline 40 & 1.4 & 1.5 & 1.5 & 1.5 & 1.5 & 1.5 & 1.6 & 1.6 & 1.6 & 1.6 & 1.6 & 1.7 & 40 \\
\hline 30 & 1.1 & 1.1 & 1.1 & 1.1 & 1.1 & 1.2 & 1.2 & 1.2 & 12 & 1.2 & 1.2 & 1.2 & 30 \\
\hline 20 & .7 & .7 & .7 & .8 & .8 & .8 & .8 & .8 & .8 & .8 & .8 & .8 & 20 \\
\hline 10 & .4 & .4 & .4 & .4 & .4 & .4 & .4 & .4 & .4 & .4 & .4 & .4 & 10 \\
\hline 9 & .3 & .3 & .3 & .3 & .3 & .3 & .4 & .4 & .4 & .4 & .4 & .4 & 9 \\
\hline 8 & .3 & .3 & .3 & 3 & .3 & .3 & .3 & .3 & .3 & .3 & .3 & .3 & 8 \\
\hline 7 & .3 & .3 & .3 & 3 & .3 & .3 & .3 & .3 & .3 & .3 & .3 & .3 & 7 \\
\hline 3 & .2 & .2 & .2 & 2 & .2 & .2 & 2 & .2 & .2 & .2 & .2 & .2 & 6 \\
\hline 5 & .2 & .2 & .2 & .2 & 2 & $\therefore$ & 2 & .2 & .2 & .2 & .2 & .2 & 5 \\
\hline 4 & 1 & .1 & .1 & 2 & .2 & 2 & .2 & .2 & .2 & 2 & .2 & .2 & 4 \\
\hline 8 & .1 & .1 & .1 & 1 & 1 & .1 & .1 & .1 & .1 & .1 & .1 & 1 & 3 \\
\hline 2 & .1 & .1 & .1 & .1 & .1 & 1 & 1 & .1 & .1 & .1 & .1 & .1 & 2 \\
\hline & & & & & & & & & $\cdots$ & & & & 1 \\
\hline 范 & 360 & & $0=0$ & & 3.80 & 3.85 & 3.90 & 3.95 & 4.00 & 4.05 & 4.10 & 4.15 & $\stackrel{4}{8}$ \\
\hline
\end{tabular}


Table VII. Pounds of fat in I to I0,000 lbs. of milk (Continued).

\begin{tabular}{|c|c|c|c|c|c|c|c|c|c|c|c|c|c|}
\hline 苞 & $|4.20|$ & 4.25 & 4.30 & 4.35 & 4.40 & 4.45 & $4.50 \mid$ & 4.55 & 4.60 & $4.65 \mid$ & 4.70 & 4.75 & 勇 \\
\hline $\begin{array}{l}\text { Milk } \\
\text { lbs. }\end{array}$ & & & & & & & & & & & & & $\begin{array}{l}\text { Milk } \\
\text { lbs. }\end{array}$ \\
\hline 10,000 & 420 & 425 & 430 & 435 & 440 & 445 & 450 & 455 & 460 & 465 & 470 & 475 & 10,000 \\
\hline & 378 & 383 & 387 & 392 & 396 & 401 & 405 & 410 & 414 & 419 & 423 & 428 & 9,000 \\
\hline 8,000 & 336 & 340 & $3+4$ & 348 & 352 & 356 & 360 & 364 & 368 & $37 \cdot$ & 376 & 380 & 8,000 \\
\hline 7,000 & 294 & 298 & 301 & 305 & 308 & 312 & 315 & 319 & 322 & 326 & 329 & 333 & 7,000 \\
\hline 6,00 & 252 & 255 & 258 & 261 & 264 & 267 & 270 & 273 & 276 & $|279|$ & 282 & 285 & 6,000 \\
\hline 000 & 210 & 213 & 215 & 218 & 220 & 223 & 225 & $2 \div 8$ & 230 & 233 & 235 & 238 & $5,0<0$ \\
\hline & 168 & 170 & $|172|$ & 174 & 176 & 178 & 180 & $18:$ & 154 & 186 & 188 & 190 & 4,000 \\
\hline 000 & 126 & 128 & 129 & 131 & 132 & 134 & 135 & \begin{tabular}{|l|}
137 \\
\end{tabular} & $\mid 138$ & \begin{tabular}{|l|}
140 \\
\end{tabular} & 141 & 143 & 3,000 \\
\hline 000 & 84.0 & 85.0 & 86.0 & 87.0 & SS.0 & 89.0 & 90.0 & 91.0 & 92.0 & 93.0 & 94.0 & 95.0 & 2,000 \\
\hline 000 & 42.0 & 42.5 & 43.0 & 43.5 & 44.0 & 44.5 & 45.0 & 45.5 & 46.0 & 46.5 & 47.0 & 47.5 & 1,000 \\
\hline & & & 38.7 & 08.2 & 39.0 & $|40.1|$ & 40.5 & 41.0 & 41.4 & 41.9 & 42.3 & 42.8 & 900 \\
\hline 800 & $\mid 33.6$ & $3+.0=$ & $|34.4|$ & & & $|35.6|$ & $36.0 \mid$ & & 36.8 & $37.2 \mid$ & 37.6 & 38.0 & 800 \\
\hline 700 & 29.4 & $29.8:$ & 30.1 & 30.5 & 30.8 & 31.2 & 31.5 & 31.9 & 32.2 & 32.6 & $32.9{ }^{2}$ & 33.3 & 700 \\
\hline 600 & 25.2 & 25.5 & 5.8 & 26.1 & 26.4 & $206.7 \mid$ & $|27.0|$ & 27.3 & $27.6]$ & 27.9 & 28.2 & 28.5 & 600 \\
\hline 500 & $\mid 21.0$ & $21.3:$ & 21.5 & & 22.0 & 22.3 & 22.5 & 22.8 & 23.0 & 23.3 & 23.5 & 23.8 & 500 \\
\hline 400 & || 16.8 & 17.0 & 17.2 & 17.4 & 17.6 & 17.8 & 18.0 & 18.2 & $\mid 18.4$ & 18.6 & 18.8 & 19.0 & 400 \\
\hline & $\mid 12.6$ & & 12.9 & 13.1 & 13.2 & 13.4 & 13.5 & 13.7 & 13.8 & 14.0 & 14.1 & 14.3 & 300 \\
\hline 200 & || 8.4 & 8.5 & 8. & 8.7 & 8.8 & $|8.9|$ & 9.0 & 9.1 & 9.2 & 9.3 & 9.4 & 9.5 & 200 \\
\hline 100 & 4.2 & 4.3 & 4.3 & 4.4 & 4.4 & 4.5 & 4.5 & 4.6 & 4.6 & 4.7 & 4.7 & 4.8 & 100 \\
\hline 90 & 3.8 & 3.8 & 3.0 & 3.9 & 4.0 & 4 & 4.1 & 4.1 & 4.1 & 4.2 & 4.2 & 4.3 & 90 \\
\hline & 3.4 & 3.4 & 3.4 & 3.5 & 3.5 & 3.6 & 3.6 & 3.6 & 3.7 & 3.7 & 3.8 & 3.8 & 80 \\
\hline 70 & 2.9 & 3.0 & 3.0 & 3.0 & 3.1 & 3.1 & 3.2 & 3.2 & 3.2 & 3.3 & 3.3 & 3.3 & 70 \\
\hline 60 & 2.5 & 2.6 & 2.6 & 2.6 & 2.6 & 2.7 & 2.7 & 2.7 & 2.8 & 2.8 & 2.8 & 2.9 & 60 \\
\hline 50 & 2.1 & 2.1 & 2.2 & 2.2 & 2.2 & 2.2 & 2.3 & 2.3 & 2.3 & 2.3 & 2.4 & 2.4 & 50 \\
\hline 40 & 1.7 & 1.7 & 1.7 & 1.7 & 1.8 & 1.8 & 1.8 & 1.8 & 1.8 & 1.9 & 1.9 & 1.9 & 40 \\
\hline 30 & 1.3 & 1.3 & 1.3 & 1.3 & 1.3 & 1.3 & 1.4 & 1.4 & 1.4 & 1.4 & 1.4 & 1.4 & 30 \\
\hline 20 & .8 & .9 & .9 & .9 & .9 & .9 & .9 & .9 & .9 & .9 & .9 & 1.0 & 20 \\
\hline 10 & .4 & .4 & .4 & .4 & .4 & .4 & .5 & .5 & .5 & .5 & .5 & .5 & 10 \\
\hline 9 & .4 & .4 & .4 & .4 & .4 & .4 & .4 & .4 & .4 & .4 & .4 & .4 & 9 \\
\hline & & .3 & .3 & .3 & .4 & .4 & .4 & .4 & .4 & .4 & .4 & & 8 \\
\hline 7 & .3 & .3 & .3 & .3 & .3 & .3 & .3 & .3 & .3 & .3 & .3 & .3 & 7 \\
\hline & & .3 & .3 & .3 & .3 & .3 &.$\hat{j}$ & .3 & .3 & .3 & .3 & .3 & 6 \\
\hline 5 & 2 & .2 & .2 & .2 & .2 & 2 & .2 & .2 & .2 & .2 & .2 & .2 & 5 \\
\hline 4 & .2 & .2 & .2 & .2 & .2 & .2 & .2 & .2 & .2 & .2 & .2 & .2 & 4 \\
\hline & 1 & .1 & .1 & .1 & .1 & .1 & .1 & .1 & .1 & .1 & .1 & .1 & 3 \\
\hline & .1 & .1 & .1 & .1 & .1 & .1 & .1 & .1 & .1 & .1 & .1 & .1 & 2 \\
\hline & & & & & & & & $\cdots$ & $\cdots$ & 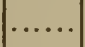 & 1 & & 1 \\
\hline & & & & & .40 & 45 & 4.50 & .55 & 4.61 & 4.65 & .7 & 4.75 & \\
\hline
\end{tabular}


Table VII. Pounds of fat in I to $\mathbf{1 0 . 0 0 0 ~ I b s . ~ o f ~ m i l k ~ ( C o n t i n u e d ) . ~}$

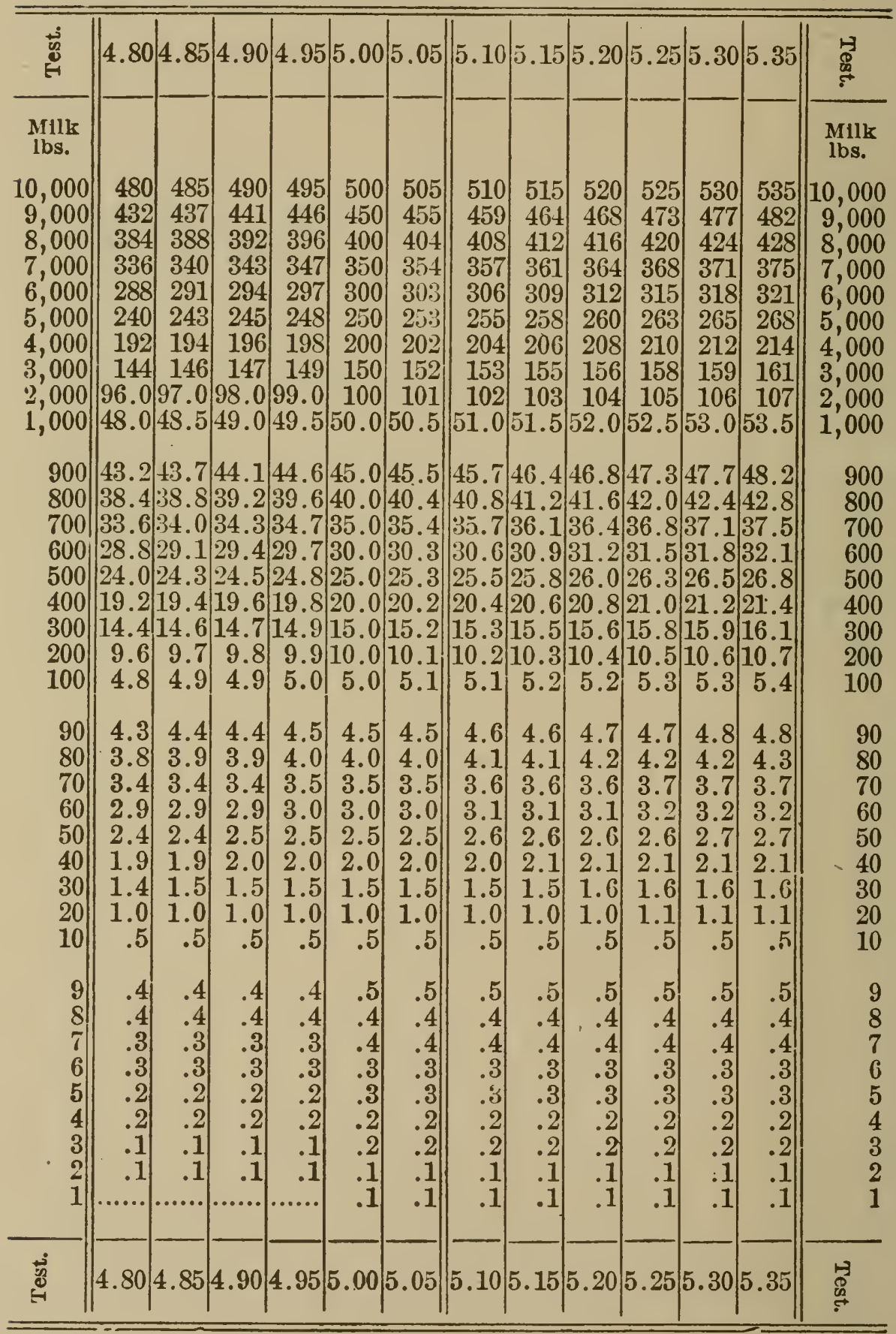


Table VIII. Pounds of fat in I to 1000 lbs. of cream testing $\mathbf{1 2 . 0}$ to $\mathbf{5 0 . 0}$ per cent. fat.

(See directions for use, p. 2(5)

\begin{tabular}{|c|c|c|c|c|c|c|c|c|c|c|c|c|c|c|c|c|c|c|c|}
\hline : & 12 & 13 & 14 & 15 & 16 & 17 & 18 & 19 & 20 & 21 & 22 & 23 & 24 & 25 & 26 & 27 & 28 & 29 & 30 \\
\hline & & & & & & & & & & & & & & & & & & & \\
\hline $\begin{array}{l}000 \\
400 \\
800 \\
700 \\
600 \\
500 \\
400 \\
800 \\
200 \\
100\end{array}$ & $\begin{array}{r}120 \\
108 \\
96 \\
84 \\
72 \\
60 \\
48 \\
36 \\
24 \\
12\end{array}$ & \begin{tabular}{|r|}
180 \\
117 \\
104 \\
91 \\
78 \\
65 \\
52 \\
39 \\
26 \\
13
\end{tabular} & \begin{tabular}{|r|}
140 \\
126 \\
112 \\
98 \\
84 \\
70 \\
56 \\
42 \\
28 \\
14
\end{tabular} & $\begin{array}{r}150 \\
135 \\
120 \\
105 \\
90 \\
75 \\
60 \\
45 \\
80 \\
15\end{array}$ & $\begin{array}{r}160 \\
144 \\
128 \\
112 \\
96 \\
80 \\
64 \\
48 \\
82 \\
16\end{array}$ & $\begin{array}{r}170 \\
153 \\
136 \\
119 \\
102 \\
85 \\
68 \\
51 \\
84 \\
17\end{array}$ & \begin{tabular}{|r|}
180 \\
162 \\
144 \\
126 \\
108 \\
90 \\
72 \\
54 \\
36 \\
18
\end{tabular} & $\begin{array}{r}190 \\
171 \\
15.2 \\
133 \\
114 \\
95 \\
76 \\
57 \\
38 \\
19\end{array}$ & $\begin{array}{r}200 \\
180 \\
160 \\
110 \\
120 \\
100 \\
80 \\
60 \\
40 \\
20\end{array}$ & \begin{tabular}{|r|}
210 \\
18.4 \\
168 \\
147 \\
126 \\
105 \\
84 \\
63 \\
42 \\
21
\end{tabular} & $\begin{array}{r}220 \\
198 \\
176 \\
151 \\
132 \\
110 \\
88 \\
66 \\
44 \\
22\end{array}$ & $\begin{array}{r}230 \\
207 \\
184 \\
161 \\
138 \\
115 \\
92 \\
69 \\
46 \\
23\end{array}$ & \begin{tabular}{|r|}
240 \\
216 \\
192 \\
163 \\
144 \\
120 \\
96 \\
72 \\
48 \\
24
\end{tabular} & $\begin{array}{r}250 \\
225 \\
200 \\
175 \\
150 \\
125 \\
100 \\
75 \\
50 \\
25\end{array}$ & $\begin{array}{c}260 \\
234 \\
208 \\
182 \\
156 \\
130 \\
104 \\
78 \\
52 \\
26\end{array}$ & \begin{tabular}{|r|}
270 \\
243 \\
216 \\
189 \\
162 \\
135 \\
108 \\
81 \\
54 \\
27
\end{tabular} & \begin{tabular}{|c|}
280 \\
252 \\
224 \\
196 \\
168 \\
140 \\
112 \\
84 \\
56 \\
28
\end{tabular} & $\begin{array}{c}290 \\
261 \\
232 \\
203 \\
174 \\
145 \\
116 \\
87 \\
58 \\
29\end{array}$ & $\begin{array}{r}300 \\
270 \\
240 \\
210 \\
180 \\
150 \\
120 \\
90 \\
60 \\
80\end{array}$ \\
\hline $\begin{array}{l}90 \\
80 \\
70 \\
60 \\
50 \\
40 \\
30 \\
20 \\
10\end{array}$ & $\begin{array}{l}9.6 \\
8.4 \\
7.2 \\
6.0 \\
4.8 \\
3.6 \\
2.4 \\
1.2\end{array}$ & $\begin{array}{l}9.1 \\
7.8 \\
6.5 \\
5.2 \\
3.9 \\
2.6 \\
1.3\end{array}$ & \begin{tabular}{l|}
9.8 \\
8.4 \\
7.0 \\
5.6 \\
4.2 \\
2.8 \\
1.4
\end{tabular} & $\begin{array}{r}10.5 \\
9.0 \\
7.5 \\
6.0 \\
4.5 \\
3.0 \\
1.5\end{array}$ & $\begin{array}{r}11.2 \\
9.6 \\
8.0 \\
6.4 \\
4.8 \\
3.2 \\
1.6\end{array}$ & \begin{tabular}{r|}
11.9 \\
10.2 \\
8.5 \\
6.8 \\
5.1 \\
3.4 \\
1.7
\end{tabular} & \begin{tabular}{r|}
16.2 \\
14.4 \\
12.6 \\
10.8 \\
9.0 \\
7.2 \\
5.4 \\
3.6 \\
1.8
\end{tabular} & $\begin{array}{r}17.1 \\
15.2 \\
13.3 \\
11.4 \\
9.5 \\
7.6 \\
5.7 \\
3.8 \\
1.9\end{array}$ & $\begin{array}{r}16.0 \\
14.0 \\
12.0 \\
10.0 \\
8.0 \\
6.0 \\
4.0 \\
2.0\end{array}$ & \begin{tabular}{r|}
14.7 \\
12.6 \\
10.5 \\
8.4 \\
6.3 \\
4.2 \\
2.1
\end{tabular} & $\begin{array}{r}15.4 \\
13.2 \\
11.0 \\
8.8 \\
6.6 \\
4.4 \\
2.2\end{array}$ & \begin{tabular}{r|}
13.8 \\
11.5 \\
9.2 \\
6.9 \\
4.6 \\
2.3
\end{tabular} & \begin{tabular}{r|}
16.8 \\
14.4 \\
12.0 \\
9.6 \\
7.2 \\
4.8 \\
2.4
\end{tabular} & $\begin{array}{r}17.0 \\
15.0 \\
12.5 \\
10.0 \\
7.5 \\
5.0 \\
2.5\end{array}$ & $\begin{array}{r}23.4 \\
20.8 \\
18.2 \\
15.6 \\
13.0 \\
10.4 \\
7.8 \\
5.2 \\
2.6\end{array}$ & \begin{tabular}{r|}
18.9 \\
16.2 \\
13.5 \\
10.8 \\
8.1 \\
5.4 \\
2.7
\end{tabular} & $\begin{array}{r}19.6 \\
16.8 \\
14.0 \\
11.2 \\
8.4 \\
5.6 \\
2.8\end{array}$ & \begin{tabular}{r|}
17.4 \\
14.5 \\
11.6 \\
8.5 \\
5.8 \\
2.9
\end{tabular} & $\begin{array}{r}27.0 \\
24.0 \\
21.0 \\
18.0 \\
15.0 \\
12.0 \\
9.0 \\
6.0 \\
3.0\end{array}$ \\
\hline $\begin{array}{l}9 \\
8 \\
7 \\
6 \\
5 \\
4 \\
8 \\
2 \\
1\end{array}$ & $\begin{array}{r}1.08 \\
.96 \\
.84 \\
.72 \\
.60 \\
.48 \\
.36 \\
.24 \\
.12\end{array}$ & $\begin{array}{r}1.17 \\
1.04 \\
.91 \\
.78 \\
.65 \\
.52 \\
.39 \\
.26 \\
.13\end{array}$ & $\begin{array}{r}1.12 \\
.98 \\
.84 \\
.70 \\
.56 \\
.42 \\
.28 \\
.14\end{array}$ & $\begin{array}{r}1.35 \\
1.20 \\
1.05 \\
.90 \\
.75 \\
.60 \\
.45 \\
.30 \\
.15\end{array}$ & $\begin{array}{l}1.28 \\
1.12 \\
.96 \\
.80 \\
.48 \\
.32 \\
.16\end{array}$ & \begin{tabular}{r|}
1.36 \\
1.19 \\
1.02 \\
.85 \\
.68 \\
.51 \\
.34 \\
.17
\end{tabular} & $\begin{array}{r}1.44 \\
1.26 \\
1.08 \\
.90 \\
.72 \\
.54 \\
.36\end{array}$ & $\begin{array}{r}1.71 \\
1.52 \\
1.33 \\
1.14 \\
.95 \\
.76 \\
.57 \\
.38 \\
.19\end{array}$ & $\begin{array}{r}1.60 \\
1.40 \\
1.20 \\
1.00 \\
.80 \\
.60 \\
.40 \\
.20\end{array}$ & $\begin{array}{l}1.89 \\
1.68 \\
1.47 \\
1.26 \\
1.05 \\
.84 \\
.63 \\
.42 \\
.21\end{array}$ & $\begin{array}{r}1.98 \\
1.76 \\
1.54 \\
1.32 \\
1.10 \\
.88 \\
.66 \\
.44 \\
.22\end{array}$ & $\begin{array}{r}2.07 \\
1.81 \\
1.61 \\
1.38 \\
1.15 \\
.92 \\
.69 \\
.46 \\
.23\end{array}$ & $\begin{array}{c}2.16 \\
1.92 \\
1.68 \\
1.44 \\
1.20 \\
.96 \\
.72 \\
.48 \\
.24\end{array}$ & $\begin{array}{l}2.25 \\
2.00 \\
1.75 \\
1.50 \\
1.25 \\
1.00 \\
.75 \\
.50 \\
.25\end{array}$ & $\begin{array}{l}2.34 \\
2.08 \\
1.82 \\
1.56 \\
1.30 \\
1.04 \\
.78 \\
.52 \\
.26\end{array}$ & $\begin{array}{l}1.43 \\
2.16 \\
1.89 \\
1.62 \\
1.35 \\
1.08 \\
.81 \\
.54 \\
.27\end{array}$ & $\begin{array}{l}2.52 \\
2.24 \\
1.96 \\
1.68 \\
1.40 \\
1.12 \\
.84 \\
.56 \\
.28\end{array}$ & $\begin{array}{l}2.61 \\
2.32 \\
2.03 \\
1.74 \\
1.45 \\
1.16 \\
.87 \\
.58 \\
.29\end{array}$ & $\begin{array}{l}2.70 \\
2.40 \\
2.10 \\
1.80 \\
1.50 \\
1.20 \\
.90 \\
.60 \\
.80\end{array}$ \\
\hline
\end{tabular}


Table VIn. Pounds of fat in 1 to 1000 lbs. of cream (continued).

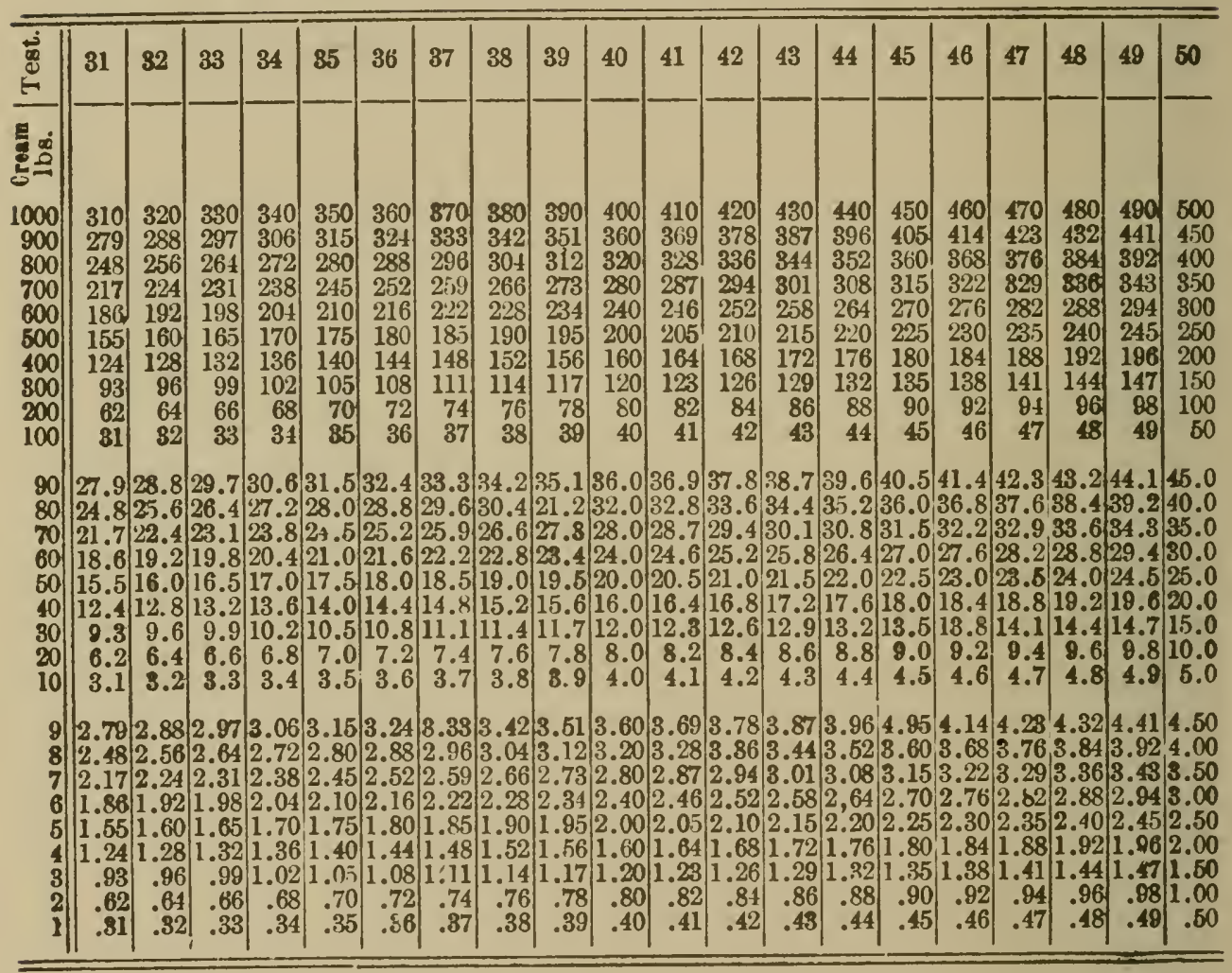


Iable IX. Amount due for butter fat, in dollars and cents, at 12 to 25 cents per pound.

(See directions for use, page 26j.)

\begin{tabular}{|c|c|c|c|c|c|c|c|c|c|c|c|}
\hline & \multicolumn{10}{|c|}{ Pounds of butter fat. } & ד \\
\hline 4 & 1,000 & 900 & 800 & 700 & 600 & 500 & 400 & 300 & 200 & 100 & 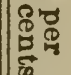 \\
\hline & $\$$ & $\$$ & $\$$ & $\$$ & $\$$ & $\$$ & $\$$ & $\$$ & $\$$ & $\$$ & \\
\hline \begin{tabular}{c|}
12 \\
124 \\
212 \\
$22 \frac{2}{4}$
\end{tabular} & $\begin{array}{l}120.00 \\
122.50 \\
125.00 \\
12.50\end{array}$ & $\left\{\begin{array}{l}108.00 \\
110.25 \\
112.50 \\
114.75\end{array}\right.$ & $\begin{array}{l}96.00 \\
98.00 \\
100.00 \\
102.00\end{array}$ & \begin{tabular}{|l}
84.00 \\
85.75 \\
87.50 \\
89.25
\end{tabular} & $\begin{array}{l}72.00 \\
73.50 \\
75.00 \\
76.50\end{array}$ & $\mid \begin{array}{l}60.00 \\
61.25 \\
62.50 \\
63.75\end{array}$ & $\mid \begin{array}{l}48.00 \\
49.00 \\
50.00 \\
51.00\end{array}$ & \begin{tabular}{|}
36.00 \\
36.75 \\
37.50 \\
38.25
\end{tabular} & $\begin{array}{l}24.00 \\
24.50 \\
25.00 \\
25.50\end{array}$ & $\left|\begin{array}{l}12.00 \\
12.25 \\
12.50 \\
12.75\end{array}\right|$ & $\begin{array}{l}12 \\
12 \\
12 \frac{1}{2} \\
12 \frac{3}{4}\end{array}$ \\
\hline \begin{tabular}{l||}
3 \\
3 \\
3 \\
$3 \frac{1}{2}$ \\
3 \\
$3 \frac{3}{4}$
\end{tabular} & $\begin{array}{l}130.00 \\
132.50 \\
135.00 \\
137.50\end{array}$ & $\left\{\begin{array}{l}17.00 \\
119.25 \\
121.50 \\
123.75\end{array}\right.$ & $\begin{array}{l}104.00 \\
106.00 \\
110.00\end{array}$ & $\begin{array}{l}91.00 \\
92.75 \\
94.50 \\
96.25\end{array}$ & $\begin{array}{l}78.00 \\
79.50 \\
81.00 \\
82.50\end{array}$ & $\begin{array}{l}65.00 \\
66.25 \\
67.50 \\
68.75\end{array}$ & $\begin{array}{l}52.00 \\
53.00 \\
54.00 \\
55.00\end{array}$ & $\begin{array}{l}39.00 \\
39.75 \\
40.50 \\
41.25\end{array}$ & $\begin{array}{l}26.00 \\
26.50 \\
27.00 \\
27.50\end{array}$ & $\left|\begin{array}{|}13.00 \\
13.25 \\
13.50 \\
13.75\end{array}\right|$ & $\mid \begin{array}{l}13 \\
13 \frac{1}{4} \\
13 \frac{1}{2} \\
13 \frac{3}{4}\end{array}$ \\
\hline $\begin{array}{l}4 \\
44 \\
44 \\
4 \frac{1}{2} \\
4 \\
4 \frac{3}{4}\end{array}$ & $\begin{array}{l}140.00 \\
142.50 \\
145.00 \\
147.50\end{array}$ & $\left\{\begin{array}{l}126.00 \\
128.25 \\
130.50 \\
132.75\end{array}\right.$ & $\begin{array}{l}112.00 \\
114.00 \\
116.00 \\
118.00\end{array}$ & $\begin{array}{r}98.00 \\
99.75 \\
101.50 \\
103.25\end{array}$ & $\begin{array}{l}84.00 \\
85.50 \\
87.00 \\
88.50\end{array}$ & $\begin{array}{l}72.25 \\
72.50 \\
73.75\end{array}$ & $\begin{array}{l}56.00 \\
57.00 \\
58.00 \\
59.00\end{array}$ & $\begin{array}{l}42.00 \\
42.75 \\
33.50 \\
44.25\end{array}$ & $\begin{array}{l}29.00 \\
29.50\end{array}$ & $\mid \begin{array}{l}14.00 \\
14.25 \\
14.50 \\
14.50\end{array}$ & $\begin{array}{l}14 \\
14 \pm \\
14 \frac{1}{2} \\
14 \frac{3}{3}\end{array}$ \\
\hline $\begin{array}{l}15 \\
15 \frac{1}{4} \\
15 \frac{1}{2} \\
15 \frac{3}{4}\end{array}$ & $\begin{array}{l}150.00 \\
152.50 \\
155.00 \\
157.50\end{array}$ & $\left\{\begin{array}{l}135.00 \\
337.25 \\
139.50 \\
141.75\end{array}\right.$ & $\begin{array}{l}120.00 \\
122.00 \\
124.00 \\
126.00\end{array}$ & $\begin{array}{l}105.00 \\
106.75 \\
108.50 \\
110.25\end{array}$ & $\begin{array}{l}90.0 \\
91.50 \\
93.00 \\
94.5\end{array}$ & $\begin{array}{l}76.25 \\
7.5 \\
7.75\end{array}$ & $\begin{array}{l}62.00 \\
63.00\end{array}$ & $\begin{array}{l}45.00 \\
45.75 \\
46.50 \\
47.25\end{array}$ & $\begin{array}{l}30.00 \\
30.50 \\
31.00 \\
31.50\end{array}$ & \begin{tabular}{|}
15.00 \\
15.25 \\
15.50 \\
15.75
\end{tabular} \mid & $\mid \begin{array}{l}15 \\
15 \\
15 \frac{1}{2} \\
153\end{array}$ \\
\hline $\begin{array}{l}16 \\
16 \frac{1}{4} \\
16 \frac{1}{2} \\
16 \frac{3}{4}\end{array}$ & $\begin{array}{l}160.00 \\
162.50 \\
165.00 \\
167.50\end{array}$ & $\begin{array}{l}144.00 \\
146.25 \\
148.50 \\
150.75\end{array}$ & $\begin{array}{l}128.00 \\
130.00 \\
132.00 \\
134.00\end{array}$ & $\begin{array}{l}112.00 \\
113.75 \\
115.50 \\
117.25\end{array}$ & $\begin{array}{l}96.00 \\
97.5 \\
99.0 \\
100.5\end{array}$ & $\begin{array}{l}81.2 \\
82.50 \\
83.7\end{array}$ & $\begin{array}{l}66.00 \\
67.00\end{array}$ & $\mid \begin{array}{l}4.00 \\
48.75 \\
49.50 \\
50.25\end{array}$ & $\mid \begin{array}{l}33.00 \\
33.50\end{array}$ & $\left|\begin{array}{l}16.00 \\
16.25 \\
16.50 \\
16.75\end{array}\right|$ & $\mid \begin{array}{l}16 \\
16 \pm \\
16 \frac{1}{2} \\
16\end{array}$ \\
\hline $\begin{array}{l}17 \\
171 \\
17 \frac{1}{2} \\
17 \frac{3}{4}\end{array}$ & $\begin{array}{l}170.00 \\
172.50 \\
175.00 \\
177.50\end{array}$ & $\begin{array}{l}153.00 \\
55.25 \\
157.50 \\
159.75\end{array}$ & $\begin{array}{l}136.00 \\
138.00 \\
140.00 \\
142.00\end{array}$ & $\begin{array}{l}119.00 \\
120.75 \\
122.50 \\
124.25\end{array}$ & $\begin{array}{l}102.0 \\
103.5 \\
105.0 \\
106.5\end{array}$ & $\begin{array}{l}87.5 \\
87.7\end{array}$ & $\begin{array}{l}70.00 \\
71.00\end{array}$ & $\mid \begin{array}{l}51.75 \\
55.50 \\
53.25\end{array}$ & 35.00 & $\begin{array}{l}17.00 \\
17.25 \\
17.50 \\
17.75\end{array}$ & $\mid \begin{array}{l}17 \\
17 \\
17 \frac{1}{2} \\
17\end{array}$ \\
\hline $\begin{array}{l}18 \\
18 \\
88\end{array}$ & $\begin{array}{l}180.00 \\
182.50 \\
185.00 \\
187.50\end{array}$ & $\begin{array}{l}162.00 \\
164.25 \\
168.50\end{array}$ & $\begin{array}{l}144.00 \\
146.00 \\
148.00 \\
150.00\end{array}$ & $\begin{array}{l}126.00 \\
127.75 \\
129.50 \\
131.25\end{array}$ & & 93.5 & \begin{tabular}{|l}
72.00 \\
73.00 \\
74.00 \\
75.00
\end{tabular} & $\begin{array}{l}54.00 \\
5.75 \\
55.50 \\
56.25\end{array}$ & $\begin{array}{l}36.50 \\
37.00 \\
37.50\end{array}$ & $\begin{array}{l}18.00 \\
18.25 \\
18.50 \\
18.75\end{array}$ & $\mid \begin{array}{l}18 \\
18 \frac{1}{2} \\
18 \frac{1}{2} \\
18 \frac{3}{4}\end{array}$ \\
\hline & 1,000 & 900 & 800 & 700 & 600 & 500 & 400 & 300 & 200 & 100 & \\
\hline
\end{tabular}


lable IX. Amount due for butter fat (Continued).

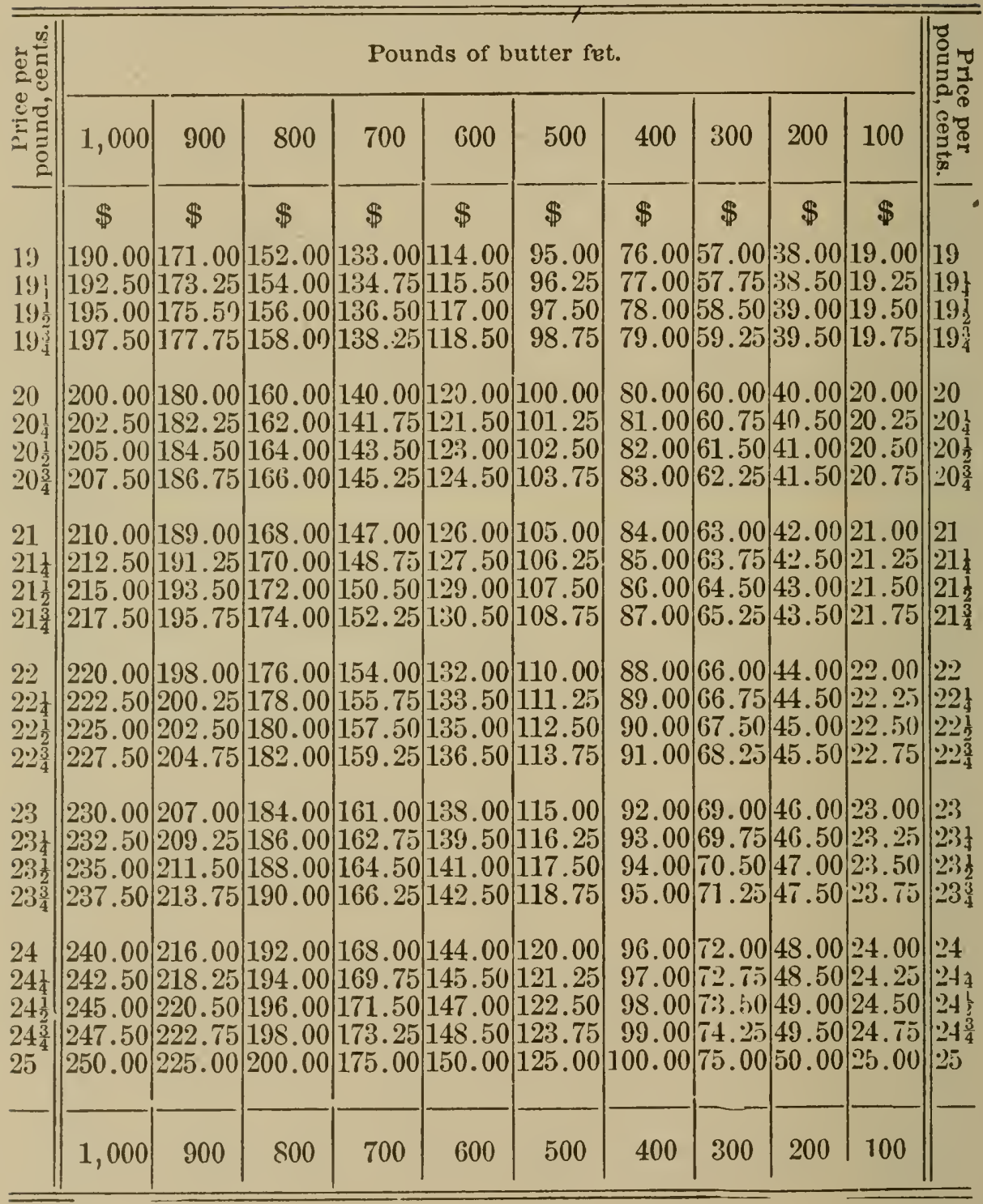


Table $X$. Relative-value tables.

(See directions for use, par. 238.)

\begin{tabular}{|c|c|c|c|c|c|c|c|c|c|c|c|}
\hline 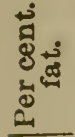 & \multicolumn{11}{|c|}{ Price of milk per 100 pounds, in dollars and cents. } \\
\hline 3.0 & .30 & .31 & .33 & .34 & .36 & .37 & .39 & .40 & .42 & .43 & \\
\hline 3.1 & .31 & .33 & .34 & .36 & .37 & .39 & .40 & .42 & .43 & .45 & \\
\hline 3.2 & .32 & .34 & .35 & .37 & .38 & .40 & .42 & .43 & .45 & .46 & \\
\hline 3.3 & .33 & .35 & .36 & .38 & .40 & .41 & .43 & .45 & .46 & .48 & .48 \\
\hline 3.4 & .34 & .36 & .37 & .39 & .41 & .42 & .44 & .46 & .48 & .49 & .51 \\
\hline 3.5 & .35 & .37 & .38 & .40 & .42 & .44 & .45 & .47 & .49 & .51 & $.5 z$ \\
\hline 3.6 & .36 & .38 & .40 & .41 & .43 & .45 & .47 & .49 & .50 & .52 & .54 \\
\hline 3.7 & .37 & .39 & .41 & .43 & .44 & .46 & .48 & .50 & .52 & .54 & .5 \\
\hline 3.8 & .38 & .40 & .42 & .44 & .46 & .47 & .49 & .51 & .53 & .55 & .5 \\
\hline 3.9 & .39 & .41 & .43 & .45 & .47 & .49 & .51 & .53 & .55 & .57 & .58 \\
\hline 4.0 & .40 & .42 & .44 & .46 & .48 & .50 & .52 & .54 & .56 & .58 & $.6 \mathrm{C}$ \\
\hline 4.1 & .41 & .43 & .45 & .47 & .49 & .51 & .53 & .55 & .57 & .59 & .61 \\
\hline .2 & .42 & .44 & .46 & .48 & .50 & .52 & .55 & .57 & .59 & .61 & $.6 E$ \\
\hline 4.3 & .43 & .45 & .47 & .49 & .53 & .54 & .56 & .58 & .60 & .62 & .64 \\
\hline 4.4 & .44 & .46 & .48 & .51 & .53 & .55 & .57 & .59 & .62 & .64 & .66 \\
\hline 4.5 & .45 & .47 & .49 & .52 & .54 & .56 & .58 & .61 & .63 & .65 & .67 \\
\hline & .46 & .48 & .51 & .53 & .55 & .57 & .60 & .62 & .64 & .67 & .66 \\
\hline 7 & .47 & .49 & .52 & .54 & .56 & .59 & .61 & .63 & .66 & .68 & $.7 C$ \\
\hline 4.8 & .48 & .50 & .53 & .55 & 58 & .60 & .62 & .65 & .67 & .70 & .72 \\
\hline 4.9 & .49 & .51 & .54 & .56 & .59 & .61 & .64 & .66 & .69 & .71 & .73 \\
\hline 5.0 & .50 & .52 & .55 & .57 & .60 & .62 & .65 & .67 & .70 & .72 & .75 \\
\hline 5. & .51 & .54 & .56 & .59 & .61 & .64 & .66 & .69 & .71 & .74 & $.7 €$ \\
\hline 5.2 & .52 & .55 & .57 & .60 & .62 & .65 & .68 & .70 & .73 & .75 & ع $7 \varepsilon$ \\
\hline 5.3 & .53 & .56 & .58 & .61 & .64 & .66 & .69 & .72 & .74 & .77 & .78 \\
\hline 5.4 & .54 & .57 & .59 & .62 & .65 & .67 & .70 & .73 & .76 & .78 & .81 \\
\hline 5.5 & .55 & .58 & .60 & .63 & .66 & .69 & .71 & .74 & .77 & .80 & $.8 \leftarrow$ \\
\hline 5.6 & .56 & .59 & .62 & .64 & .67 & .70 & .73 & .76 & .78 & .81 & .84 \\
\hline 5.7 & .57 & .60 & .63 & .66 & .68 & .71 & .74 & .77 & .80 & .83 & .85 \\
\hline 5.8 & .58 & .61 & .64 & .67 & .70 & .72 & .75 & .78 & .81 & .84 & .87 \\
\hline 5.9 & .59 & .62 & .65 & .68 & .71 & .74 & .77 & .80 & .83 & .86 & .88 \\
\hline 6.0 & .60 & .63 & .66 & .69 & .72 & .75 & .78 & .81 & .84 & .87 & .80 \\
\hline
\end{tabular}


Table X. Relative-value tables (Continued).

\begin{tabular}{|c|c|c|c|c|c|c|c|c|c|c|}
\hline ¿ే. & \multicolumn{10}{|c|}{ Price of millk per 100 pounds, in dollars and cents. } \\
\hline 3.0 & 46 & .48 & .49 & .51 & .52 & .54 & .55 & .57 & .58 & .60 \\
\hline 3.1 & .48 & .50 & .51 & .53 & .54 & .56 & .57 & .59 & .60 & .62 \\
\hline 3.2 & .50 & .51 & .53 & .54 & .56 & .58 & .59 & .61 & .62 & .64 \\
\hline 3.3 & .51 & .53 & .54 & .56 & .58 & .59 & .61 & .63 & .64 & .66 \\
\hline 3.4 & .53 & .54 & .56 & .58 & .59 & .61 & .63 & .65 & .66 & .68 \\
\hline 3.5 & .54 & .56 & .58 & .69 & .61 & .63 & .65 & .66 & .68 & .70 \\
\hline 3.6 & .56 & .68 & .59 & .01 & .63 & .65 & .67 & .68 & .70 & .72 \\
\hline 8.7 & .57 & .59 & .61 & .69 & .65 & .67 & .68 & .70 & .72 & .74 \\
\hline 3.8 & .59 & .61 & .63 & .65 & .66 & .68 & .70 & .72 & .74 & .76 \\
\hline 8.9 & .60 & .62 & .64 & .66 & .68 & .70 & .72 & .74 & .76 & .78 \\
\hline 4.0 & .62 & .64 & .66 & .68 & .70 & .72 & .74 & .76 & .78 & .80 \\
\hline 4.1 & .64 & .66 & .68 & .70 & .72 & .74 & .76 & .78 & .80 & .82 \\
\hline 4.2 & .65 & .67 & .69 & .71 & .73 & .76 & .78 & .80 & .82 & .84 \\
\hline 4.3 & .67 & .69 & .71 & .73 & $: 75$ & .77 & .80 & .82 & .84 & .86 \\
\hline 4.4 & .68 & .70 & .73 & .75 & .77 & .79 & .81 & .84 & .86 & .88 \\
\hline 4.5 & .70 & .72 & .74 & .76 & .79 & .81 & .83 & .85 & .88 & .90 \\
\hline 4.6 & .71 & .74 & .76 & .78 & .80 & .83 & .85 & .87 & .90 & .92 \\
\hline 4.7 & .73 & .75 & .78 & .80 & .82 & .85 & .87 & .89 & .92 & .94 \\
\hline 4.8 & .74 & .77 & .79 & .82 & .84 & .86 & .89 & .91 & .94 & .96 \\
\hline 4.9 & .76 & .78 & .81 & .83 & .86 & .88 & .91 & .93 & .96 & .98 \\
\hline 5.0 & .77 & .80 & .82 & .85 & .87 & .90 & .92 & .95 & .97 & 1.00 \\
\hline 5.1 & .79 & .82 & .84 & .87 & .89 & .92 & .94 & .97 & .99 & 1.02 \\
\hline 5.2 & .81 & .83 & .86 & .88 & .91 & .94 & .96 & .99 & 1.01 & 1.04 \\
\hline 5.3 & .83 & .85 & .87 & .90 & .93 & .95 & .98 & 1.01 & 1.03 & 1.06 \\
\hline 5.4 & .84 & .86 & .89 & .92 & .94 & .97 & 1.00 & 1.03 & 1.05 & 1.08 \\
\hline 5.5 & .85 & .88 & .91 & .93 & .96 & .99 & 1.02 & 1.04 & 1.07 & 1.10 \\
\hline 5.6 & .87 & .90 & .92 & .95 & .98 & 1.01 & 1.04 & 1.06 & 1.09 & 1.12 \\
\hline 5.7 & .88 & .91 & .94 & .97 & 1.00 & 1.03 & 1.05 & 1.08 & 1.11 & 1.14 \\
\hline 5.8 & .90 & .93 & .96 & .99 & 1.01 & 1.04 & 1.07 & 1.10 & 1.13 & 1.16 \\
\hline 5.9 & .91 & .94 & .97 & 1.00 & 1.03 & 1.06 & 1.09 & 1.12 & 1.15 & 1.18 \\
\hline 6.0 & .93 & .96 & .99 & 1.02 & 1.05 & 1.08 & 1.11 & 1.14 & 1.17 & 1.20 \\
\hline
\end{tabular}


Appendix.

Table X. Relative-value tables (Continued).

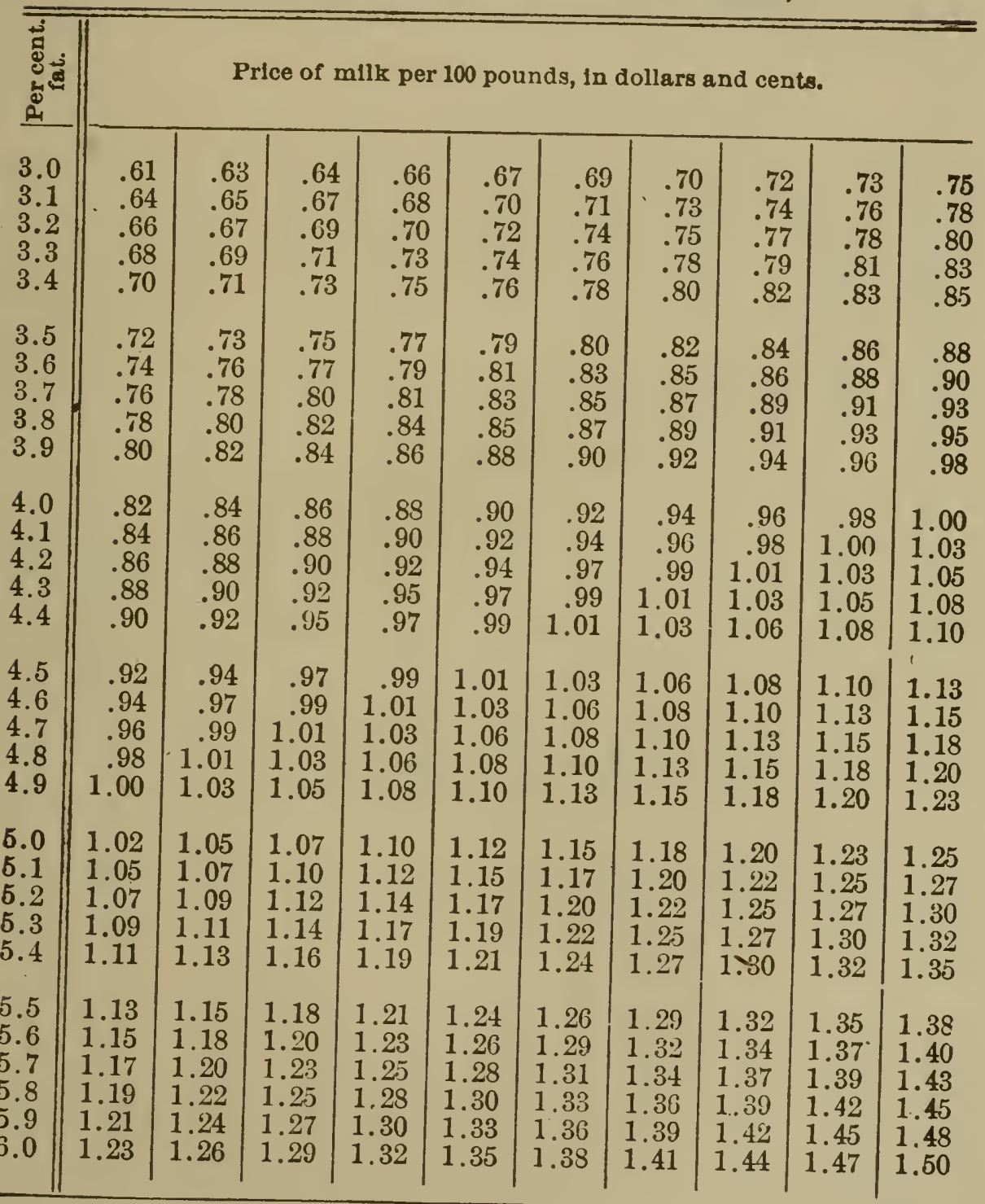


Table X. Relative-value tables (Continued).

\begin{tabular}{|c|c|c|c|c|c|c|c|c|c|c|}
\hline 0 & \multicolumn{10}{|c|}{ Price of milk por 100 pounds, in dollars and cents. } \\
\hline & .76 & .78 & .79 & .81 & .82 & .84 & .85 & .87 & .88 & .90 \\
\hline 1 & .79 & .81 & .82 & .84 & .85 & .87 & .88 & .90 & .91 & .93 \\
\hline & .82 & .83 & .85 & .86 & .88 & .90 & .91 & .93 & .94 & .96 \\
\hline 3 & .84 & .86 & .87 & .89 & .91 & .92 & .94 & .96 & .97 & .99 \\
\hline & .87 & .88 & .90 & .92 & .93 & .95 & .97 & .99 & 1.00 & 1.02 \\
\hline & .89 & .91 & .93 & .94 & .96 & .98 & 1.00 & 1.01 & 1.03 & 1.05 \\
\hline & .92 & .94 & .95 & .97 & .99 & 1.00 & 1.03 & 1.04 & 1.06 & 1.08 \\
\hline & .94 & .96 & .98 & 1.00 & 1.02 & 1.03 & 1.05 & 1.07 & 1.09 & 1.11 \\
\hline & .07 & .99 & 1.01 & 1.03 & 1.04 & 1.06 & 1.08 & 1.10 & 1.12 & 1.14 \\
\hline 3.9 & .99 & 1.01 & 1.03 & 1.05 & 1.07 & 1.09 & 1.11 & 1.13 & 1.15 & 1.17 \\
\hline & 1.02 & 1.04 & 1.06 & 1.08 & 1.10 & 1.12 & 1.14 & 1.16 & 1.18 & 1.20 \\
\hline & 1.05 & 1.07 & 1.09 & 1.11 & 1.13 & 1.15 & 1.17 & 1.19 & 1.21 & 1.23 \\
\hline & 1.07 & 1.09 & 1.11 & 1.13 & 1.15 & 1.18 & 1.20 & 1.22 & 1.24 & 1.26 \\
\hline & 1.10 & 1.12 & 1.14 & 1.16 & 1.18 & 1.20 & 1.23 & 1.25 & 1.27 & 1.29 \\
\hline 4 & 1.12 & 1.14 & 1.17 & 1.19 & 1.21 & 1.23 & 1.25 & 1.28 & 1.30 & 1.32 \\
\hline 4 & 1.15 & 1.17 & 1.19 & 1.21 & 1.24 & 1.26 & 1.28 & 1.30 & 1.33 & 1.35 \\
\hline & 1.17 & 1.20 & 1.22 & 1.24 & 1.26 & 1.29 & 1.31 & 1.33 & 1.36 & 1.38 \\
\hline & 1.20 & 1.22 & 1.25 & 1.27 & 1.29 & 1.32 & 1.34 & 1.36 & 1.39 & 1.41 \\
\hline & 1.22 & 1.25 & 1.27 & 1.30 & 1.32 & 1.34 & 1.37 & 1.39 & 1.42 & 1.44 \\
\hline & 1.25 & 1.27 & 1.30 & 1.32 & 1.35 & 1.37 & 1.40 & 1.42 & 1.45 & 1.47 \\
\hline & 1.27 & 1.30 & 1.32 & 1.35 & 1.37 & 1.40 & 1.42 & 1.45 & 1.47 & 1.50 \\
\hline & 1.30 & 1.33 & 1.35 & 1.38 & 1.40 & 1.43 & 1.45 & 1.48 & 1.50 & 1.53 \\
\hline & 1.33 & 1.35 & 1.37 & 1.40 & 1.43 & 1.46 & 1.48 & 1.51 & 1.53 & 1.56 \\
\hline & 1.35 & 1.38 & 1.40 & 1.43 & 1.46 & 1.48 & 1.51 & 1.54 & 1.56 & 1.59 \\
\hline & 1.38 & 1.40 & 1.43 & 1.46 & 1.48 & 1.51 & 1.54 & 1.57 & 1.59 & 1.62 \\
\hline & 1.40 & 1.43 & 1.46 & 1.48 & 1.51 & 1.54 & 1.57 & 1.60 & 1.62 & 1.65 \\
\hline & 1.43 & 1.46 & 1.48 & 1.51 & 1.54 & 1.57 & 1.60 & 1.62 & 1.65 & 1.68 \\
\hline & 1.45 & 1.48 & 1.51 & 1.54 & 1.57 & 1.60 & 1.62 & 1.65 & 1.68 & 1.71 \\
\hline & 1.48 & 1.51 & 1.54 & 1.57 & 1.59 & 1.62 & 1.65 & 1.68 & 1.71 & 1.74 \\
\hline & 1.50 & 1.53 & 1.56 & 1.59 & 1.62 & 1.65 & 1.68 & 1.71 & 1.74 & 1.77 \\
\hline 6. & 1.53 & 1.56 & 1.59 & 1.62 & 1.65 & 1.68 & 1.71 & 1.74 & 1.77 & 1.80 \\
\hline
\end{tabular}


Iable XI. Butter chart, showing calculated yield of butter (in Ibs.) from I to 10,000 lbs. of milk, testing 3.0 to 5.3 per cent. (See directions for use, p. 265.)

\begin{tabular}{|c|c|c|c|c|c|c|c|c|c|c|c|c|c|}
\hline 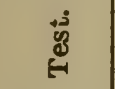 & .00 & 3.10 & 3.20 & 3.30 & 3.40 & 3.50 & 3.60 & 3.70 & 3.80 & 3.90 & 4.00 & 4.10 & $\begin{array}{l}\text { 悤 } \\
\stackrel{8}{*}\end{array}$ \\
\hline $\begin{array}{c}\text { Milk, } \\
\text { lbs. }\end{array}$ & & & & & & & & & & & & & $\begin{array}{l}\text { Milk, } \\
\text { lbs. }\end{array}$ \\
\hline 0,000 & 325 & 336 & 348 & 360 & 371 & 383 & 394 & 406 & 418 & 429 & 441 & 452 & 10,000 \\
\hline 000 & 293 & 302 & 313 & 324 & 334 & 345 & 355 & 365 & 376 & 386 & 397 & 407 & 9,0 \\
\hline 00 & 260 & 269 & 278 & 288 & 297 & 306 & 315 & 325 & 334 & 343 & 353 & 362 & \\
\hline & 228 & $2: 35$ & 244 & 252 & 260 & 268 & 276 & 284 & 293 & 300 & 309 & 316 & \\
\hline 6,000 & 195 & 202 & 209 & 216 & 223 & 230 & 236 & 244 & 251 & 257 & 265 & 271 & \\
\hline 000 & 163 & 168 & 174 & 180 & 186 & 192 & 197 & 203 & 209 & 215 & 221 & 226 & \\
\hline 4,000 & 130 & 134 & 139 & 144 & 148 & 153 & 158 & 162 & 167 & 172 & 176 & 181 & \\
\hline 3,000 & 97.5 & 101 & 104 & 108 & 111 & 115 & 118 & 122 & 125 & 129 & 132 & 136 & \\
\hline 2,000 & 65.0 & 67.2 & 69.6 & 72.0 & 74.2 & 76.6 & 78.8 & 81.2 & 83.6 & 85.8 & 88.2 & 90.4 & 2, \\
\hline 1,000 & 32.5 & 33.6 & 34.8 & 36.0 & 37.1 & 38.3 & 39.4 & 40.6 & 41.8 & 43.9 & 44.1 & 45.2 & 1,000 \\
\hline 900 & 9.3 & 30.2 & 31.3 & $32.4 \mid$ & $|33.4|$ & 34.5 & $35.5 \mid$ & 36.5 & $\mid 37.6$ & 38.6 & 39.7 & 40.7 & 900 \\
\hline 800 & 26.0 & 26.9 & 27.8 & 28.8 & 29.7 & 30.6 & 31.5 & 32.5 & 33.4 & 34.3 & 35.3 & 36.2 & 300 \\
\hline 700 & 22.8 & 23.5 & 24.4 & 25.2 & 26.0 & 26.8 & 27.6 & 28.4 & 29.3 & 30.0 & 30.9 & 31.6 & 00 \\
\hline 600 & 19.5 & 20.2 & 20.9 & 21.6 & 22.3 & 23.0 & 23.6 & 24.4 & 25.1 & 25.7 & 26.5 & 27.1 & 600 \\
\hline 500 & 16.3 & 16.8 & 17.4 & 18.0 & 18.6 & 19.2 & 19.7 & 20.3 & 20.9 & 21.5 & 22.1 & 22.6 & 500 \\
\hline 400 & 13.0 & 13.4 & 13.9 & 14.4 & 14.8 & 15.3 & 15.8 & 16.2 & 16.7 & 17.2 & 17.6 & 18.1 & 100 \\
\hline 300 & 9.7 & 10.1 & 10.4 & 10.8 & 11.1 & 11.5 & 11.8 & 12.2 & 12.5 & 12.9 & 132 & 13.6 & 300 \\
\hline 200 & 6.5 & 6.7 & 6.9 & 7.2 & 7.4 & 7.6 & 7.9 & 8.1 & 8.3 & 8.6 & 8.8 & 9.0 & 200 \\
\hline 100 & 3.2 & 3.4 & 3.5 & 3.6 & 3.7 & 3.8 & 3.9 & 4.1 & 4.2 & 4.3 & 4.4 & 4.5 & 100 \\
\hline 90 & 2.9 & 3.0 & 3.1 & 3.2 & 3.3 & 3.4 & 3.5 & 3.6 & 3.7 & 3.8 & 3.9 & & 90 \\
\hline 80 & 2.6 & 2.7 & 2.8 & 2.9 & 3.0 & 3.1 & 3.2 & 3.3 & 34 & 34 & 35 & Q & 80 \\
\hline 70 & 2.3 & 2.3 & 2.4 & 2.5 & 2.6 & 2.7 & 28 & 28 & 29 & 30 & 31 & 32 & 70 \\
\hline 60 & 1.9 & 2.0 & 2.1 & 2.2 & 2.2 & 23 & 2.4 & 24 & 25 & 26 & 27 & 27 & 60 \\
\hline 50 & 1.6 & 1.7 & 1.7 & 1.8 & 1.9 & 1.8 & 2.0 & 2.0 & 2.1 & 2.2 & 22 & 23 & 50 \\
\hline 40 & 1.3 & 1.3 & 1.4 & 1.4 & 1.5 & 1.5 & 1.6 & 1. & 1.7 & 1.7 & 1.8 & 1.8 & 40 \\
\hline 30 & 1.0 & 1.0 & 1.0 & 1.1 & 1.1 & 1.2 & 1.2 & 1.2 & 1.3 & 1.3 & 1.3 & 1.4 & 30 \\
\hline 20 & .6 & .7 & .7 & .7 & .7 & .8 & .8 & .8 & .8 & .9 & .9 & .9 & 20 \\
\hline 10 & 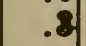 & & .4 & .4 & .4 & .4 & .4 & .4 & .4 & .4 & .4 & .5 & 10 \\
\hline 8 & .8 & .3 & .3 & .3 & .3 & .3 & .4 & .4 & .4 & .4 & .4 & .4 & 9 \\
\hline 8 &. & .3 & .3 & .3 & .3 & .3 & .3 & .3 & .3 & .3 & .4 & .4 & 8 \\
\hline 7 & .2 & .2 & .2 & .3 & .3 & .3 & .3 & .3 & .3 & .3 & .3 & .3 & \\
\hline 6 & .2연 & .2 & .2 & .2 & .2 & .2 & .2 & .2 & .3 & .3 & .3 & .3 & \\
\hline 5 & .2 & .2 & .2 & .2 & .2 & .2 & .2 & .2 & .2 & .2 & .2 & .2 & 5 \\
\hline 4 & 1 & 1 & 1 & .2 & .2 & .2 & .2 & .2 & .2 & .2 & .2 & .2 & 4 \\
\hline 8 & .1 & 1 & .1 & .14 & .1 & .1 & .1 & .1 & 1 & .1 & 1 & .1 & 8 \\
\hline 2 & .1 & .1 & .1 & .1 & .1 & .1 & .1 & .1 & .1 & .1 & .1 & 1 & \\
\hline & & & & & & & & & & & & & \\
\hline$\vec{z}$ & & & & & 40 & 50 & 3.60 & 3.70 & & 3.90 & 4.00 & 4.10 & \\
\hline
\end{tabular}


Tabie il. Butter chart (Continued).

\begin{tabular}{|c|c|c|c|c|c|c|c|c|c|c|c|c|c|}
\hline 莒 & 4.20 & 4.30 & 4.40 & 4.50 & 4.60 & 4.70 & 4.80 & 4.90 & 5.00 & 5.10 & 5.20 & 5.30 & $\begin{array}{l}{ }_{0} \\
\mathbb{0} \\
\mathfrak{L}^{+}\end{array}$ \\
\hline $\begin{array}{l}\text { Milk } \\
\text { lbs. }\end{array}$ & & & & & & & & & & & & & $\begin{array}{l}\text { Milk } \\
\text { lbs. }\end{array}$ \\
\hline 10,000 & 464 & 476 & 487 & 499 & 510 & 522 & 534 & 545 & 557 & 568 & 580 & 592 & 10,000 \\
\hline 9,000 & 418 & 428 & 438 & 449 & 459 & 470 & 481 & 491 & 501 & 511 & 522 & 533 & 9,000 \\
\hline 8,000 & 371 & 381 & 390 & 399 & 408 & 418 & 427 & 436 & 446 & 454 & 464 & 474 & 8,000 \\
\hline 7,000 & 325 & 333 & 341 & 349 & 357 & 365 & 374 & 382 & 390 & 398 & 406 & 414 & 7,000 \\
\hline 6,000 & 278 & 286 & 292 & 299 & 306 & 313 & 320 & 327 & 334 & 341 & 348 & 355 & 6,000 \\
\hline 5,000 & 232 & 238 & 244 & 250 & 255 & 261 & 267 & 273 & 279 & 284 & 290 & 296 & 5,000 \\
\hline 4,000 & 186 & 190 & 195 & 200 & 204 & 209 & 214 & 218 & 223 & 227 & 232 & 237 & 4,000 \\
\hline 3,000 & 139 & 143 & 146 & 150 & 153 & 157 & 160 & 164 & 167 & 170 & 174 & 178 & 3,000 \\
\hline 2,000 & $92.8 \mid$ & 95.2 & 97.4 & 99.8 & 102 & 104 & 107 & 109 & 111 & 114 & 116 & 118 & 2,000 \\
\hline 1,000 & 46.4 & 47.6 & 48.7 & 49.9 & 51.0 & 52.2 & 53.4 & 54.5 & 55.7 & 56.8 & 58.0 & 59.2 & 1,000 \\
\hline 900 & 41.8 & 42.8 & 43.8 & 44.9 & 45.9 & 47.0 & 48.1 & 49.1 & 50.1 & $|51.1|$ & 52.2 & ว3.3 & 900 \\
\hline 800 & 37.1 & 38.1 & 39.0 & 39.9 & 40.8 & 41.8 & 42.7 & 43.6 & 44.6 & 45.4 & 46.4 & 47.4 & 800 \\
\hline 700 & 32.5 & 33.3 & 34.1 & 34.9 & 35.7 & 36.5 & 37.4 & 38.2 & 39.0 & 39.8 & 40.6 & 41.4 & 700 \\
\hline 600 & $27.8 \mid$ & 28.6 & 29.2 & 29.9 & 30.6 & 31,3 & 32.0 & 32.7 & 33.4 & 34.1 & 34.8 & 35.5 & 600 \\
\hline 500 & $\mid 23.2$ & 23.8 & 24,4 & 25.0 & 25.5 & 26.1 & 26.7 & 27.3 & 27.9 & 28,4 & 29.0 & 29.6 & 500 \\
\hline 400 & 18.6 & 19.0 & 19.5 & 20.0 & 20.4 & 20.9 & 21.4 & 21.8 & 22.3 & 22.7 & 23.2 & 23.7 & 400 \\
\hline 300 & $|13.9|$ & 14.3 & 14.6 & 15.0 & 15.3 & 15.7 & $|16.0|$ & $|16.4|$ & 16.7 & 17.0 & 17.4 & 17.8 & 300 \\
\hline 200 & 9.3 & 9.5 & 9.7 & 10.0 & 10.2 & 10.4 & 10.7 & 10.9 & 11.1 & 11.4 & 11.6 & 11.8 & 200 \\
\hline 100 & 4.6 & 4.8 & 4.9 & 5.0 & 5.1 & 5.2 & 5.3 & 5.5 & 5.6 & 5.7 & 5.8 & 5.9 & 100 \\
\hline 90 & 4.2 & 4.3 & 4.4 & 4.5 & 4.6 & 4.7 & 4.8 & 4.9 & 5.0 & 5.1 & 5.2 & 5.3 & 90 \\
\hline 80 & 3.7 & 3.8 & 3.9 & 4.0 & 4.1 & 4.2 & 4.3 & 4.4 & 4.5 & 4.5 & 4.6 & 4.7 & 80 \\
\hline 70 & 3.3 & 3.3 & 3.4 & 3.5 & 3.6 & 3.7 & 3.7 & 3.8 & 3.9 & 4.0 & 4.1 & 4.1 & 70 \\
\hline 60 & 2.5 & 2.9 & 2.9 & 3.0 & 3.1 & 3.1 & 3.2 & 3.3 & 3.3 & 3.4 & 3.5 & 3.6 & 60 \\
\hline 50 & 2.3 & 2.4 & 2.4 & 2.5 & 2.6 & 2.6 & 2.7 & 2.7 & 2.8 & 2.8 & 2.9 & 3.0 & 50 \\
\hline 40 & 1.9 & 1.9 & 2.0 & 2.0 & 2.0 & 2.1 & 2.1 & 2.2 & 2.2 & 2.3 & 2.3 & 2.4 & 40 \\
\hline 30 & 1.4 & 1.4 & 1.5 & 1.5 & 1.5 & 1.6 & 1.6 & 1.6 & 1.7 & 1.7 & 1.7 & 1.8 & 30 \\
\hline 20 & .9 & 1.0 & 1.0 & 1.0 & 1.0 & 1.0 & 1.1 & 1.1 & 11 & 1.1 & 1.2 & 1.2 & 20 \\
\hline 10 & .5 & .5 & .5 & .5 & .5 & .5 & .5 & .6 & .6 & .6 & .6 & .6 & 10 \\
\hline 9 & .4 & .4 & .4 & .5 & .5 & .5 & .5 & .5 & .5 & .5 & .5 & .5 & 9 \\
\hline 8 & .4 & .4 & .4 & .4 & .4 & .4 & .4 & .4 & .5 & .5 & .5 & .5 & 8 \\
\hline 7 & .3 & .3 & .3 & .4 & .4 & .4 & .4 & .4 & .4 & .4 & .4 & .4 & 7 \\
\hline 6 & .3 & .3 & .3 & .3 & .3 & .3 & .3 & .3 & .3 & .3 & .4 & .4 & 6 \\
\hline 5 & .2 & .2 & .2 & .3 & .3 & .3 & .3 & .3 & .3 & .3 & .3 & .3 & 5 \\
\hline 4 & .2 & .2 & .2 & .2 & .2 & .2 & .2 & .2 & .2 & .2 & .2 & .2 & 4 \\
\hline 3 & .1 & .1 & .2 & .2 & .2 & .2 & .2 & .2 & .2 & .2 & .2 & .2 & 3 \\
\hline 2 & .1 & .1 & .1 & .1 & .1 & .1 & .1 & .1 & .1 & .1 & .1 & .1 & 2 \\
\hline 1 & .1 & .1 & .1 & .1 & .1 & .1 & .1 & .1 & .1 & 1 & .1 & .1 & 1 \\
\hline 泀 & 4.20 & 4.30 & 4.40 & 4.50 & 4.60 & 4.70 & 4.80 & 4.90 & $5 . C 0$ & 5.10 & 5.20 & 5.30 & 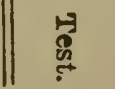 \\
\hline
\end{tabular}


Table XII. Overrun table, showing pounds of butter from one hundred lbs. of milk. (See directions for use, par. 222.)

\begin{tabular}{|c|c|c|c|c|c|c|c|c|c|c|c|c|}
\hline $\begin{array}{l}\text { Per } \\
\text { cent. } \\
\text { fat. }\end{array}$ & 1.10 & 1.11 & 1.12 & 1.13 & 1.14 & 1.15 & 1.16 & 1.17 & 1.18 & 1.19 & 1.20 & $\begin{array}{c}\text { Per } \\
\text { cent. } \\
\text { fat. }\end{array}$ \\
\hline $\begin{array}{l}3.0 \\
3.1 \\
3.2 \\
3.3 \\
3.4\end{array}$ & $\begin{array}{l}3.30 \\
3.41 \\
3.52 \\
3.63 \\
3.74\end{array}$ & $\begin{array}{l}3.33 \\
3.44 \\
3.55 \\
3.66 \\
3.77\end{array}$ & $\left|\begin{array}{l}3.36 \\
3.47 \\
3.58 \\
3.70 \\
3.81\end{array}\right|$ & $\left|\begin{array}{l}3.39 \\
3.50 \\
3.62 \\
3.73 \\
3.84\end{array}\right|$ & $\left|\begin{array}{l}3.42 \\
3.53 \\
3.65 \\
3.76 \\
3.85\end{array}\right|$ & $\begin{array}{l}3.45 \\
3.57 \\
3.65 \\
3.50 \\
3.91\end{array}$ & $\begin{array}{l}3.48 \\
3.60 \\
3.71 \\
3.83 \\
3.94\end{array} \mid$ & $\begin{array}{l}3.51 \\
3.63 \\
3.74 \\
3.86 \\
3.98\end{array}$ & $\begin{array}{l}3.54 \\
3.66 \\
3.78 \\
3.89 \\
4.01\end{array}$ & $\mid \begin{array}{l}3.57 \\
3.68 \\
3.81 \\
3.93 \\
4.05\end{array}$ & $\begin{array}{l}3.60 \\
3.72 \\
3.84 \\
3.96 \\
4.08\end{array}$ & $\begin{array}{l}3.0 \\
3.1 \\
3.2 \\
3.3 \\
3.4\end{array}$ \\
\hline $\begin{array}{l}3.5 \\
3.6 \\
3.7 \\
3.8 \\
3.9\end{array}$ & $\begin{array}{l}3.85 \\
3.96 \\
4.07 \\
4.18 \\
4.29\end{array}$ & $\begin{array}{l}3.89 \\
4.00 \\
4.11 \\
4.22 \\
4.33\end{array}$ & $\mid \begin{array}{l}4.03 \\
4.14 \\
4.26 \\
4.37\end{array}$ & $\begin{array}{l}3.96 \\
4.07 \\
4.15 \\
4.29 \\
4.41\end{array}$ & $\begin{array}{l}3.99 \\
4.10 \\
4.22 \\
4.33 \\
4.45\end{array}$ & $\begin{array}{l}4.03 \\
4.14 \\
4.26 \\
4.2 \\
4.49\end{array}$ & $\begin{array}{l}4.06 \\
4.18 \\
4.28 \\
4.41 \\
4.52\end{array} \mid$ & $\mid \begin{array}{l}4.10 \\
4.21 \\
4.33 \\
4.45 \\
4.56\end{array}$ & $\begin{array}{l}4.13 \\
4.25 \\
4.37 \\
4.48 \\
4.60\end{array}$ & $\begin{array}{l}4.17 \\
4.28 \\
4.40 \\
4.52 \\
4.64\end{array}$ & $\begin{array}{l}4.20 \\
4.32 \\
4.44 \\
4.56 \\
4.68\end{array}$ & $\begin{array}{l}3.5 \\
3.6 \\
3.7 \\
3.8 \\
3.9\end{array}$ \\
\hline $\begin{array}{l}4.0 \\
4.1 \\
4.2 \\
4.3 \\
4.4\end{array}$ & $\begin{array}{l}4.40 \\
4.51 \\
4.62 \\
4.73 \\
4.84\end{array}$ & $\begin{array}{l}4.44 \\
4.55 \\
4.66 \\
4.77 \\
4.88\end{array}$ & $\begin{array}{l}4.48 \\
4.59 \\
4.70 \\
4.82 \\
4.93\end{array}$ & $\begin{array}{l}4.52 \\
4.63 \\
4.75 \\
4.86 \\
4.97\end{array}$ & $\begin{array}{l}4.56 \\
4.67 \\
4.79 \\
4.90 \\
5.02\end{array}$ & $\begin{array}{l}4.60 \\
4.72 \\
4.83 \\
4.9 \overline{2} \\
5.06\end{array}$ & $\begin{array}{l}4.64 \\
4.76 \\
4.87 \\
4.99 \\
5.10\end{array}$ & $\mid \begin{array}{l}4.68 \\
4.80 \\
4.91 \\
5.03 \\
5.15\end{array}$ & $\begin{array}{l}4.72 \\
4.84 \\
4.96 \\
5.07 \\
5.19\end{array}$ & $\mid \begin{array}{l}4.76 \\
4.88 \\
5.00 \\
5.12 \\
5.24\end{array}$ & $\begin{array}{l}4.80 \\
4.92 \\
5.04 \\
5.16 \\
5.28\end{array}$ & $\begin{array}{l}4.0 \\
4.1 \\
4.2 \\
4.3 \\
4.4\end{array}$ \\
\hline $\begin{array}{l}4.5 \\
4.6 \\
4.7 \\
4.8 \\
4.9\end{array}$ & $\begin{array}{l}4.95 \\
5.06 \\
5.17 \\
5.28 \\
5.39\end{array}$ & $\begin{array}{l}5.11 \\
5.22 \\
5.33 \\
5.44\end{array}$ & $\mid \begin{array}{l}5.15 \\
5.26 \\
5.38 \\
5.49\end{array}$ & $\begin{array}{l}5.20 \\
5.31 \\
5.42 \\
5.54\end{array}$ & $\begin{array}{l}5.13 \\
5.24 \\
5.36 \\
5.47 \\
5.59\end{array}$ & $\begin{array}{l}5.29 \\
5.41 \\
5.5 \circ \\
5.64\end{array}$ & $\begin{array}{l}5.22 \\
5.34 \\
5.45 \\
5.57 \\
5.68\end{array}$ & $\begin{array}{l}5.38 \\
5.49 \\
5.62 \\
5.73 \\
5.73\end{array}$ & $\begin{array}{l}5.31 \\
5.43 \\
5.55 \\
5.66 \\
5.78\end{array}$ & $\mid \begin{array}{l}5.36 \\
5.47 \\
5.59 \\
5.71 \\
5.83\end{array}$ & $\begin{array}{l}5.40 \\
5.52 \\
5.64 \\
5.76 \\
5.88\end{array}$ & $\begin{array}{l}4.5 \\
4.6 \\
4.7 \\
4.8 \\
4.9\end{array}$ \\
\hline $\begin{array}{l}5.0 \\
5.1 \\
5.2 \\
5.3 \\
5.4\end{array}$ & $\begin{array}{l}5.50 \\
5.61 \\
5.72 \\
5.83 \\
5.84\end{array}$ & $\begin{array}{l}5 \\
5 \\
5.65 \\
5.77 \\
5.88 \\
5.99\end{array}$ & $\mid \begin{array}{l}5.60 \\
5.71 \\
5.82 \\
5.94 \\
6.05\end{array}$ & \begin{tabular}{|l}
5.65 \\
5.76 \\
5.88 \\
5.99 \\
6.10
\end{tabular} & $\mid$\begin{tabular}{|}
5.70 \\
5.81 \\
5.93 \\
6.04 \\
6.16
\end{tabular} & $\begin{array}{l}5.75 \\
5.87 \\
5.95 \\
6.10 \\
6.21\end{array}$ & $\mid \begin{array}{l}5.80 \\
5.92 \\
6.03 \\
6.15 \\
6.26\end{array}$ & $\mid \begin{array}{l}5.85 \\
5.97 \\
6.08 \\
6.20 \\
6.32\end{array}$ & $\mid \begin{array}{l}5.90 \\
6.02 \\
6.14 \\
6.25 \\
6.37\end{array}$ & $\begin{array}{l}5.95 \\
6.07 \\
6.19 \\
6.31 \\
6.43\end{array}$ & $\begin{array}{l}6.00 \\
6.12 \\
6.24 \\
6.36 \\
6.48\end{array}$ & $\begin{array}{l}5.0 \\
5.1 \\
5.6 \\
5.3 \\
5.4\end{array}$ \\
\hline $\begin{array}{l}5.5 \\
5.6 \\
5.7 \\
5.8 \\
5.9 \\
6.0\end{array}$ & $\begin{array}{l}6.05 \\
6.16 \\
6.27 \\
6.38 \\
6.49 \\
6.6 \mathrm{C}\end{array}$ & $\begin{array}{l}6.11 \\
6.22 \\
6.33 \\
6.44 \\
6.55 \\
6.66\end{array}$ & $\left|\begin{array}{l}6.16 \\
6.27 \\
6.38 \\
6.50 \\
6.61 \\
6.7 \cdot 2\end{array}\right|$ & $\begin{array}{l}6.22 \\
6.33 \\
6.44 \\
6.55 \\
6.67 \\
6.75\end{array}$ & $\left|\begin{array}{l}0.35 \\
6.50 \\
6.61 \\
6.73 \\
6.84\end{array}\right|$ & $\begin{array}{l}6.41 \\
6.56 \\
6.67 \\
6.79 \\
6.90\end{array}$ & $\left|\begin{array}{l}6.38 \\
6.50 \\
6.61 \\
6.73 \\
6.84 \\
6.96\end{array}\right|$ & $\mid \begin{array}{l}6.44 \\
6.55 \\
6.67 \\
6.79 \\
6.90 \\
7.002\end{array}$ & $\left|\begin{array}{l}6.49 \\
6.61 \\
6.73 \\
6.84 \\
6.96 \\
7.08\end{array}\right|$ & $\left|\begin{array}{l}6.55 \\
6.66 \\
6.78 \\
6.90 \\
7.02 \\
7.14\end{array}\right|$ & $\mid \begin{array}{l}6.60 \\
6.72 \\
6.84 \\
6.96 \\
7.08 \\
7.20\end{array}$ & $\begin{array}{l}5.5 \\
5.6 \\
5.7 \\
5.8 \\
5.9 \\
6.0\end{array}$ \\
\hline
\end{tabular}


Table XIII. Yield of Cheese from $100 \mathrm{lbs}$. milk with 2.5 to 6 per cent of fat, and lactometer readings from 26 to 30. (Ste par. 224)

\begin{tabular}{|c|c|c|c|c|c|c|c|c|c|c|c|c|}
\hline \multirow{2}{*}{ 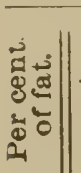 } & \multicolumn{11}{|c|}{ 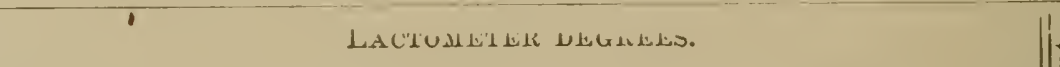 } & \multirow{2}{*}{ 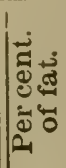 } \\
\hline & 26 & 27 & 28 & 29 & 30 & 31 & 32 & 33 & 34 & 35 & 36 & \\
\hline & 7.28 & 7.41 & 7.54 & 7.67 & 7.81 & 7.94 & 8.07 & 8.20 & 8.33 & 8.47 & 8.60 & 2.5 \\
\hline & 7.44 & 7.57 & 7.70 & 7.83 & 7.96 & 8.119 & 8.22 & 8.35 & 8.49 & 8.62 & 8.76 & 2.6 \\
\hline 2.7 & 7.59 & 7.72 & 7.85 & 7.99 & $8.1 \%$ & 8.25 & 8.38 & 8.51 & 8.64 & 8.77 & 8.91 & 2.7 \\
\hline 2.8 & 7.74 & 7.87 & 8.00 & 8.14 & 8.27 & $8.4(1)$ & 8.53 & 8.67 & 8.80 & 8.94 & 9.07 & 2.8 \\
\hline 2.9 & 7.90 & 8.03 & 8.16 & 8.30 & 8.44 & 8.56 & 8.69 & 8.82 & 8.95 & $9 .(49$ & 9.22 & 2.9 \\
\hline 3.0 & 8.05 & 8.18 & 8.31 & 8.45 & 8.58 & 8.71 & 8.84 & 8.97 & 9.11 & 9.24 & y. 37 & 3.0 \\
\hline 3.1 & 8.21 & 8.34 & 8.47 & 8.60 & 8.74 & 8.87 & 9.00 & 9.13 & 9.26 & 9.39 & 9.53 & 3.1 \\
\hline 3.2 & 8.36 & 8.44 & 8.62 & 8.75 & 8.89 & 9.02 & 9.15 & 9.28 & 9.42 & 9.55 & 9.68 & 2 \\
\hline 3.3 & 8.52 & 8.65 & 8.78 & 8.91 & 9.05 & 9.18 & 9.31 & 9.44 & 9.57 & 9.70 & 9.84 & .3 \\
\hline 3.4 & 8.67 & 8.80 & 8.93 & 9.06 & 9.20 & 9.33 & 9.46 & 9.59 & 9.73 & 9.86 & 9.99 & .4 \\
\hline 3.5 & 8.82 & 8.96 & 9.09 & 9.22 & 9.35 & 9.48 & 9.62 & 9.75 & 9.88 & 10.01 & 10.15 & 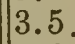 \\
\hline .6 & 8.98 & 9.11 & 9.24 & 9.37 & 9.50 & 9.63 & 9.77 & 9.90 & 10.03 & 17 & .30 & 3.6 \\
\hline 3.7 & 9.13 & 9.26 & 9.39 & 9.52 & 9.65 & 9.78 & 9.92 & $18 .(15$ & 10.19 & 32 & 46 & 3.7 \\
\hline $3 . \succ$ & 9.29 & 9.42 & 9.55 & 9.68 & 9.81 & 9.94 & $111.0 n$ & $10.2 \mathrm{~N}$ & 10.34 & & 61 & .8 \\
\hline 3.9 & 9.44 & $9.5 i$ & 9.70 & 9.84 & 9.97 & 10.10 & 10.23 & 10.36 & 50 & & & .9 \\
\hline 4. & 9.60 & 9.73 & 9.86 & 10.00 & 10.13 & 10.26 & 10.39 & 10.53 & 66 & & .93 & .0 \\
\hline & 9.75 & 9.88 & 10.02 & 0.1 & 10.28 & 10.39 & 10.54 & 10.68 & .81 & 10. & .08 & .1 \\
\hline & & 10.03 & 10.17 & 10.30 & 10.43 & 10.57 & 10.70 & 10.84 & 10.97 & 11.10 & 11.24 & .2 \\
\hline & & & 10. & & & .72 & 10.8 & 10.99 & 11.12 & 11.25 & .39 & .3 \\
\hline & & & 10.4 & & 4 & 0.87 & 11. & 11.14 & 11.27 & 11.41 & 1.55 & .4 \\
\hline & & & & & & & 11.16 & 11.29 & 11.42 & 11.56 & 11.70 & .5 \\
\hline & & & & & & 11.18 & 11. & 11.45 & 11.58 & 11.71 & 11.85 & 4.6 \\
\hline & & & & & 20 & 11.34 & 11.47 & 11.60 & 11.73 & 11.87 & 12.01 & 4.7 \\
\hline & & & & & 11.3 & 11.49 & 11. & 11.76 & 89 & 12.02 & 12.16 & 4.8 \\
\hline & & & & & 11.51 & 11.65 & 11.78 & 11.91 & & .18 & 12.32 & 4.9 \\
\hline & & & & 11. & 11.6 & 11.80 & 11.93 & 12.07 & 12.20 & 12.34 & 12.48 & ). 0 \\
\hline & & & & & 11.82 & 11.96 & 12. & 12.23 & 12.36 & 12.49 & 12.63 & $\mid 5.1$ \\
\hline & & & & & 11.98 & 12.1 & 12.24 & 12.38 & 12.52 & 12.66 & 12.80 & 5.2 \\
\hline & & & & 11 & .13 & 12.27 & 12.40 & 12.53 & 12.67 & 12.71 & 12.85 & 5.3 \\
\hline . & & & & 12 & 12.29 & 12.42 & 12.55 & 12.69 & 12.83 & 12.97 & 13.01 & 5.4 \\
\hline & & & & 12.31 & 12.44 & 12.58 & & 12.85 & 12.99 & 13.12 & 13.25 & 5.5 \\
\hline & & & 12 & 12 & 12. & 12.73 & & 13.00 & 13.14 & 13.28 & 13.41 & 5.6 \\
\hline & & & & 12.62 & 12.75 & 12.89 & 13.02 & 13.16 & 13.30 & 13.44 & 13.57 & 5.7 \\
\hline & & & & & 12.91 & 13.05 & 13. & 13.31 & & 13.59 & 13.72 & 5.8 \\
\hline & & & & & & 13.19 & 13.33 & & & & 13.87 & 5.9 \\
\hline i. 1 & $12.0^{3} y$ & & 12.95 & 13.09 & 13.22 & 13.35 & 13.49 & 13.62 & 13.75 & 13.89 & 14.02 & 6.0 \\
\hline
\end{tabular}


Iable XIV. Comparisons of Fahrenheit and Centigrade (Celsius) thermometer scales.

\begin{tabular}{|c|c|c|c|c|c|}
\hline $\begin{array}{l}\text { Fahren- } \\
\text { heit. }\end{array}$ & $\begin{array}{l}\text { Centi- } \\
\text { grade. }\end{array}$ & $\begin{array}{c}\text { Fahren- } \\
\text { heit. }\end{array}$ & $\begin{array}{c}\text { Centl- } \\
\text { grade. }\end{array}$ & $\begin{array}{l}\text { Fahren- } \\
\text { heit. }\end{array}$ & $\begin{array}{l}\text { Centi- } \\
\text { grado. }\end{array}$ \\
\hline+212 & +100 & +176 & +80 & +140 & +60 \\
\hline 211 & 99.44 & 175 & 79.44 & 139 & 59.44 \\
\hline 210 & 98.89 & 174 & 78.89 & 138 & 58.89 \\
\hline 209 & 98.33 & 173 & 78.33 & 137 & 58.33 \\
\hline 208 & 97.78 & 172 & 77.78 & 136 & 57.78 \\
\hline 207 & 97.22 & 171 & 77.22 & 135 & 57.22 \\
\hline 206 & 96.67 & 170 & 76.67 & 134 & 56.67 \\
\hline 205 & 96.11 & 169 & 76.11 & 133 & 56.11 \\
\hline 204 & 95.55 & 168 & 75.55 & 132 & 55.55 \\
\hline 203 & 95 & 167 & 75 & 131 & 55 \\
\hline 202 & 94.44 & 166 & 74.44 & 130 & 54.44 \\
\hline 201 & 93.89 & 165 & 73.89 & 129 & 53.89 \\
\hline 200 & 93.33 & 164 & 72.33 & 128 & 53.33 \\
\hline 199 & 92.78 & 163 & 72.78 & 127 & 52.78 \\
\hline 198 & 92.22 & 162 & 71.22 & 126 & 52.22 \\
\hline 197 & 91.67 & 161 & 71.67 & 125 & 51.67 \\
\hline 196 & 91.11 & 160 & 71.11 & 124 & 51.11 \\
\hline 195 & 90.55 & 159 & 70.55 & 123 & 50.55 \\
\hline 194 & 90 & 158 & 70 & 122 & 50 \\
\hline 193 & 89.44 & 157 & 69.44 & 121 & 49.44 \\
\hline 192 & 88.89 & 156 & 68.89 & 120 & 48.89 \\
\hline 191 & 88.33 & 155 & 68.33 & 119 & 48.33 \\
\hline 190 & 87.78 & 154 & 67.78 & 118 & 47.78 \\
\hline 189 & 87.22 & 153 & 67.22 & 117 & 47.22 \\
\hline 188 & 86.67 & 152 & 66.67 & 116 & 46.67 \\
\hline 187 & 86.11 & 151 & 66.11 & 115 & 46.11 \\
\hline 186 & 85.55 & 150 & 65.55 & 114 & 45.55 \\
\hline 185 & 85 & 149 & 65 & 113 & 45 \\
\hline 184 & 84.44 & 148 & 64.44 & 112 & 44.44 \\
\hline 183 & 83.89 & $1 \div 7$ & 63.89 & 111 & 43.89 \\
\hline 182 & 83.33 & 146 & 63.33 & 110 & 43.33 \\
\hline 181 & 82.78 & 145 & 62.78 & 109 & 42.78 \\
\hline 180 & 82.22 & 144 & 62.22 & 108 & 42.22 \\
\hline 179 & 81.67 & 143 & 61.67 & 107 & 41.67 \\
\hline 178 & 81.11 & 142 & 61.11 & 106 & 41.11 \\
\hline 177 & 80.55 & 141 & 60.55 & 105 & 40.55 \\
\hline
\end{tabular}


Table XIV. Comparisons of thermometer scales (Continued.)

\begin{tabular}{|c|c|c|c|c|c|}
\hline $\begin{array}{l}\text { Fahren- } \\
\text { heit. }\end{array}$ & $\begin{array}{l}\text { Centi- } \\
\text { grade. }\end{array}$ & $\begin{array}{c}\text { Fahren- } \\
\text { heit. }\end{array}$ & $\begin{array}{l}\text { Centi- } \\
\text { grade. }\end{array}$ & $\begin{array}{c}\text { Fahren- } \\
\text { heit. }\end{array}$ & $\begin{array}{l}\text { Cent1- } \\
\text { grade. }\end{array}$ \\
\hline+104 & +40 & +68 & +20 & +32 & +0 \\
\hline 103 & 39.44 & 67 & 19.44 & 31 & -0.55 \\
\hline 102 & 38.89 & 66 & 18.89 & 30 & 1.11 \\
\hline 101 & 38.33 & 65 & 18.33 & 29 & 1.67 \\
\hline 100 & 37.78 & 64 & 17.78 & 28 & 2.22 \\
\hline 99 & 37.22 & 63 & 17.22 & 27 & 2.78 \\
\hline 98 & 36.67 & 62 & 16.67 & 26 & 3.33 \\
\hline 97 & 36.11 & 61 & 16.11 & 25 & 3.89 \\
\hline 96 & 35.55 & 60 & 15.55 & 24 & 4.44 \\
\hline 95 & 35 & 59 & 15 & 23 & 5 \\
\hline 94 & 34.44 & 58 & 14.44 & 22 & 5.55 \\
\hline 93 & 33.89 & 57 & 13.89 & 21 & 6.11 \\
\hline 92 & 33.33 & 56 & 13.33 & 20 & 6.67 \\
\hline 91 & 32.78 & 55 & 12.78 & 19 & 7.22 \\
\hline 90 & 3222 & 54 & 12.22 & 18 & 7.78 \\
\hline 89 & 31.67 & 53 & 11.67 & 17 & 8.33 \\
\hline 88 & 31.11 & 52 & 11.11 & 16 & 8.89 \\
\hline 87 & 30.55 & 51 & 10.55 & 15 & 9.44 \\
\hline 86 & 30 & 50 & 10 & 14 & $10^{.7 x}$ \\
\hline 85 & 29.44 & 49 & 9.44 & 13 & 10.55 \\
\hline 84 & 28.89 & 48 & 8.89 & 12 & 11.11 \\
\hline 83 & 28.33 & 47 & 8.33 & 11 & 11.67 \\
\hline 82 & 27.78 & 46 & 7.78 & 10 & 12.22 \\
\hline 81 & 27.22 & 45 & 7.22 & 9 & 12.78 \\
\hline 80 & 26.67 & 44 & 6.67 & 8 & 13.33 \\
\hline 79 & 26.11 & 43 & 6.11 & 7 & 13.89 \\
\hline 78 & 25.55 & 42 & 5.55 & 6 & 14.44 \\
\hline 77 & 25 & 41 & 5 & 5 & 15.00 \\
\hline 76 & 24.44 & 40 & 4.44 & 4 & 15.55 \\
\hline 75 & 23.89 & 39 & 3.89 & 3 & 16.11 \\
\hline 74 & 23.33 & 38 & 3.33 & 2 & 16.67 \\
\hline 73 & 22.78 & 37 & 2.78 & 1 & 17.22 \\
\hline 72 & 22.22 & 36 & 2.22 & 0 & 17.78 \\
\hline 71 & 21.67 & 35 & 1.67 & -1 & 18.33 \\
\hline 70 & 21.11 & 34 & 1.11 & 2 & 18.89 \\
\hline 69 & 20.55 & 33 & 0.55 & 3 & 19.44 \\
\hline
\end{tabular}

To convert deg. Fahrenheit to corresponding deg. Centigrade: Subtract 32 , multiply difference by 5 , and divide by 9 .

Example: Which degree Centigrade corresponds to $110^{\circ} \mathrm{F} . ? 110-82=$ $78 ; 78 \times 5=390 ; 390+9=43.33$.

To convert deg. Centigrade to corresponding deg. Fahrenheit:

Multiply by 9 , divide product by 5 , and add 32 to quotient.

Example: Which degree Fahrenheit corresponds to $95.5^{\circ}$ C.? $95.5 \times 9=$ 859.5; $859.5+5=171.9 ; 171.9+32=203.6$. 
Table XV. Comparison of metric and customary weights and measures.

\begin{tabular}{|c|c|c|c|}
\hline $\begin{array}{l}\text { Customary } \\
\text { weights and } \\
\text { measures. }\end{array}$ & $\begin{array}{l}\text { Equivalents in } \\
\text { metric system. }\end{array}$ & $\begin{array}{l}\text { Metric weights } \\
\text { and } \\
\text { measures. }\end{array}$ & $\begin{array}{l}\text { Equivalents in } \\
\text { customary system. }\end{array}$ \\
\hline 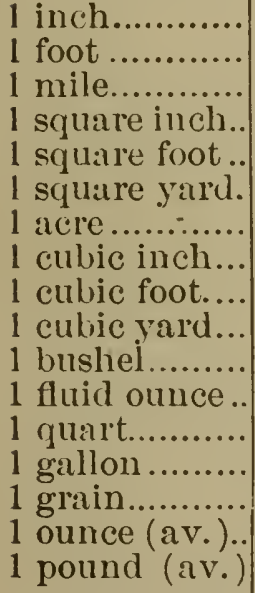 & $\begin{array}{l}2.54 \text { centimeters. } \\
.30+8 \text { meter. } \\
1.6094 \text { lilometers. } \\
6.452 \text { sq. centimeters. } \\
9.29 \text { sq. lecimeters. } \\
.836 \text { sq. meter. } \\
.4047 \text { hectare. } \\
16.387 \text { cc. } \\
.0283 \text { cub. meter. } \\
.765 \text { cub. meter } \\
.3552 \text { hectolites } \\
29.57 \text { ce. } \\
.9464 \text { liter. } \\
3.7854 \text { liters. } \\
64.8 \text { milligrans. } \\
28.35 \text { grams. } \\
.4536 \text { kilogram. }\end{array}$ & 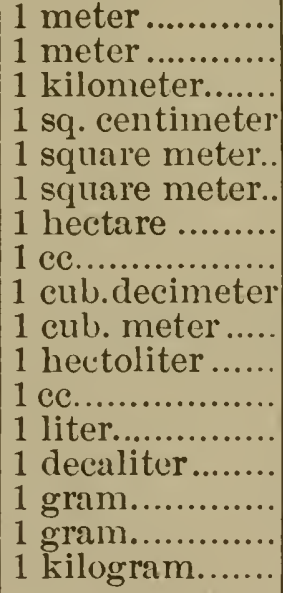 & $\begin{array}{l}39.37 \text { inches. } \\
1.0936 \text { yards. } \\
.6214 \text { mile. } \\
.155 \text { sq. inch. } \\
10.764 \text { sq. feet. } \\
1.196 \text { sq. yards. } \\
2.471 \text { acres. } \\
.061 \text { cubic inch. } \\
61.023 \text { cubic inches. } \\
35.314 \text { cub. feet. } \\
2.8377 \text { bushels. } \\
.0338 \text { fluid ounce. } \\
1.0567 \text { quarts. } \\
2.6417 \text { quarts. } \\
15.43 \text { grains. } \\
.035274 \text { ounce. } \\
2.2046 \text { pounds (av. }\end{array}$ \\
\hline
\end{tabular}




\section{SUGGESIIONS regarding the organization of co-operative creameries and cheese factories.}

When the furmers of a neighborhood are considering the establishment of a creamery or cheese factory, they should first of all make an accurate canvas of the locality to ascertain the number of cows that can be depended on to supply the factory with milk. The area which may be drawn from will vary according to the kind of factory which it is desired to operate. A successful separator creamery will need at least 400 cows within a radius of four to five miles from the proposed factory. ${ }^{1}$ Small cheese factories can be operated with less milk, and gathered-cream and butter factories generally cover a much laryer territory than that meutioned. In all cases, however, the question of the number of cows contributing to the enterprise must be fully settled before further steps are taken, since this is a point upon which success will largely depend.

Methods of organization. The farmers should form their own organization, and not accept articles of agreement proposed by traveling agents. An agreement to supply milk from i stated number of cows should be signed by all expecting to join the association. When a sufficient number of cows has been pledged to insure the successful operation of a factory, the farmers agreeing to supply milk should meet and form an organization. This may be done according to either of the following plans which have been known to give good satisfaction.

\section{Raising money for building and equipment.}

First.-Each member will sign an agreement to pay on or before a given date for a certain number of shares in the company at.........dollars per share; or,

S'econd.-An elected board of directors may be authorized to borrow a sum of money not exceeding.........thousand dollars on their individual responsibility, and the sum of........cents, (usually five cents) per hundred pounds of milk received at the factory shall be reserved for the payment of this borrowed money.

1 Bull. 56, Wisconsin experiment station. 
Constitution and by-laws of a co-operative association are drawn up and signed by the prospective members of the association when it has been determined to form such an association. It is impossible to include in an illustration all the articles and rules that may be found useful in each particular instance; the following suggestions in regard to some of the points to be included in the documents are given as a guide only. It may be found advisable to modify them in various ways to meet the needs of the organization to be formed.

After the constitution and by-laws have been drawn up and made plain to all the members of the association, they should be printed and copies distributed to all parties interested.

\section{Constitution}

OR

ARTICles of AGREEMENT OF THE.................Association. ${ }^{1}$

1. The undersigned, residents within the Counties of.........., State of............, hereby agree to become members of the Co-operative Association, which is formed for the purpose of manufacturing butter or cheese from whole milk.

2. The regular meetings of the association shall be held annually on the.............day of the month of................. Special meetings may be called by the president, or on written request of one-third of the members of the association, provided three day's notice of such meeting is sent to all members.

Meetings of the board of directors may be called in the same way, either by the president or by any two members of the board of directors.

3. Ten members of the association, or three of the board of directors, shall constitute a quorum for the transaction of business.

4. The officers of the association shall include president, secretary, treasurer, one of whom is also elected manager, and these officers together with three other members of the associa-

1 The following publications have been freely used in preparing this constitution and by-laws: Woll, Handbook f. Farmers and Dairymen; Minn. experiment station, bull. No. 35; Ontario Agriculture College, spec
tal bulletin, May 1897 . 
tion shall constitute the board of directors. Each of these six officers shall be elected at the annual meeting and hold office for one year, or until their successors have been elected and qualified. Any vacancies in the board of directors may be filled by the directors until the next annual meeting of the association.

5. The duties of the president shall be to preside at all meetings of the association, and perform the usual duties of such presiding officers. He shall sign all drafts and documents of any kind relating to the business of -the association, and pay all money which comes into his possession by virtue of his office, to the treasurer, taking his receipt therefor. He shall call special meetings of the association when deemed necessary.

In the absence of the president, one of the board of directors shall temporarily fill the position.

6. The secretary shall attend all business meetings of the association and of the board of directors and shall keep a careful record of the minutes of the meetings. He shall also give notices of all meetings and all appointments on committees, etc. He shall sign all papers issued, conduct the correspondence and general business of the association, and keep a correct financial account between the association and itsmembers. He shall have charge of all property of the association not otherwise disposed of, give bonds for the faithful performance of his duties, and receive such compensation for his servizes as the board of directors may determine.

7. The treasurer shall receive and give receipt for all money belonging to the assuciation, and pay out the same upon orders signed by the president and the secretary. He shall give such bonds as the board of directors may require.

8. The board of directors shall audit the accounts of the association, invest its funds, appoint agents, and determine all compensations. They shall prescribe and enforce the rules and regulations of the factory. They shall cause to be kept a record of the weights and tests of the milk or cream received from each patron, ${ }^{6}$. . shall divide among the patrons the money due them each month. They shall also make some provision for the with- 
drawal of any member from the association, and make a report in detail to the association at the annual meeting. Such report shall include the gross amount of milk handled during the year, the receipts from products sold, and all other receipts, the amounts paid for milk and for running expenses, and a complete statement of all other matters pertaining to the business of the association.

9. Among the rules and regulations to be enforced by the board of directors may be included some or all of the following:

a. Patrons shall furnish all the milk from all the cows promised at organization of the association.

b. Only sweet and pure milk will be accepted at the factory, and any tainted or sour milk shall be refused.

c. The milk of each patrou shall be tested at least three times a month.

d. Any patron proved to be guilty of watering, skimming or otherwise adulterating the milk sent to the factory, or by taking more than 80 pounds of skim milk or whey for every 100 pounds of whole milk delivered to the factory, shall be fined as agreed by the association.

e. A partron's premises may be inspected at any time by the board of directors, or their authorized agent, for the purpose of suggesting improvements in the methods of caring for the milk or the cows, in draiuage and general cleanliness; or to secure samples of the milk of his cows for examination when it is deemed necessary.

10. Any changes or amendments to the by-laws or constitution of the association must be made in writing by the parties proposing the same, and posted prominently in a conspicuous place at the creamery, at least two weeks previous to their being acted upon. Such changes to be in force must be adopted by $a$ two-thirds vote of the stockholders.

11. In voting at any annual or special meeting of the association, the members shall be entitled to one vote for each cow supplying milk to the factory, or for each share of the stock owned by them, as agreed upon. 



\section{INDEX}

The figures refer to pages in the book.

Acid bottle, Swedish, 47 .

Acid measures, $31,46,54$.

Acid tester, Swedish, 67.

Acidimeter, Devarda's, 124.

Acidity of cream, 125,129 ; estimation of, 135.

Acidity of milk, cause of, 119 ; determination of, 132 ; methods of testing, 120.

Acidity pellets, 125 .

Adulteration of milk, 111, 115 , 244 ; calculation of, 115 .

Adulterated butter, 240, 242 ; cheese, 245.

Albumen, 14 ; determination of, in milk, 225.

Albuminoids, 13.

Albumose, 14.

Alkaline tablet test, 124 ; standard solution of, 126 ; accuracy, 128.

Alkaline tabs, 136.

American cheddar cheese, 21.

Ames method for determining water in butter, 237.

Amphoteric reaction of milk, 119. Amyl alcohol, use in cream testing, 88 .

Analysis, chemical, of butter, 231 ; butter milk, 228; cheese, 243 ; condensed milk, 229; cream, 228 ; milk, 217 ; skim milk, 228 ; whey, 228.

Appendix, 258.

Artificial butter, detection of, 240 .

Ash, determination of, in butter, 232 ; in cheese, 244; in milk, 227.
Babcock test, the, 4, 28; Bartlett's modification, 72; directions for, 23; discussion of details, 37 ; for butter, 96 ; for butter milk, 94 ; for cheese, 97 : for condensed milk, 98 ; for cream, 75, 180; for ice-cream, 100 ; for skim milk, 90 ; for whey, 94; glassware used in, 37 ; modifications of, 71 ; scales for weighing cream, cheese, etc., S0, 233 ; water to be used in, 68 . Babcock testers, 54 ; electrical, 63 ; hand testers, 60 ; power testers, 61 ; steam turbine, 61.

Bartlett's modification of Babcock test, 48, 72 .

Bausch and Lomb centrifuge, 72 . Beimling test, 5 .

Bi-carbonate of soda, detection of, 251.

Bi-chromate of potash, 108, 168; solution of, 108.

Blended milk, 254.

Board of health degrees, 106.

Boiled milk, detection of, 249 .

Boiling test, the, 242.

Boracic acid in dairy products, 136, 251.

Borax in dairy products, 251.

Butter, artificial, 12 ; detection of, 240.

Butter chart, 278 ; use of, 197.

Butter, 20 ; Babcock test for, 196 ; chemical analysis of, 231 ; complete analysis in same sample, 232 ; composition of, 21, 258; creamery methods of estimating 
water in, 233 ; definition, 255 ; determination of ash, 232 ; casein, 231 ; fat, 231 ; salt, 233,239 ; water, 231 ; rapld estimation of water, 233; Ames method, 237 ; Cornell test, 237 ; Dean's method, 237 ; Gray's method, 235 ; Irish test, 237 ; Mitchell-Walker test, 236; Patrick's method, 236; Wisconsin high-pressure oven method, 238 ; process, 255 : renovated, 255; sampling for analysis, 95,231 ; scales for weighing, 81, 233; standard, 255 ; variations in composition, 188 ; yield, calculation of, 187.

Butter fat, amount due, at 12-25 cents per lb., 272 ; conversion factor for, 196 ; definition, 255 ; determination of specific gravity, 240 ; volatile fatty acids, 241 ; expansion coefficient, 36 ; specific gravity, 38; standard, 255 ; test and yield of butter, 187.

Butter making, quantities of products obtained in, 21.

Butter milk, 21 ; Babcock test for, 94 ; chemical analysis of, 228 ; composition, 258; definition, 255 ; specific gravity, 229.

Calculation of adulteration of milk, 115; of concentration of condensed milk, 230; of milk solids, 109 ; of overrun, 195 ; of sp. gr. of milk solids, 113 ; of yield of butter, 187, 196, 197; of cheese, 199 ; of dividends at creameries, 203 ; at cheese factories, 213 ; of percentages, 172 .

Calibration of glassware, 48 ; Trowbridge method, 51.

Carbohydrates, 15.

Casein, 13; determination of, in butter, 231 ; in cheese, 243 ; in milk, 3, 226; Hart's method, 226.

Centrifugal machines, 54 .
Chamberiand filters, 14.

Cheddar cheese, American, 21 ; composition, 258.

Cheese, 21 ; Babcock test for, 97 ; calculating yield of, from casein and fat, 201; from fat, 199 ; from solids not fat and fat, 200 ; composition, 21, 258; chemical analysis of, 243 ; definitions, 256 ; determination of ash, 244 ; casein, 243 ; fat, 243 ; water, 243 ; "filled," detection of, 244 ; quality of, from milk of different richness, 211 ; sampling, 97 ; standard, 256 ; yield, calculation of, 199 ; yield of, from milk with 2.5 to 6 per cent. fat and lactometer readings from 26 to 36,281 ; yield of, and quality of milk, relation between, 200 .

Cheese factories, calculating dividends at, 213 ; co-operative, 216 , 285 ; proprietary, 215.

Cholesterin in milk, 18.

Citric acid in milk, 18.

Cleaning solution for test bottles, 43.

Cleaning test 'bottles, 40 ; apparatus for, 41,44 .

Cochran's test, 5 .

Coloring matter in milk, detection of, 247.

Colostrum milk, 18 ; composition of, 258.

Combined acid bottle, 47 .

Composite samples, 151 ; care of, 170 ; case for holding, 166 ; methods of taking, 160 ; preservatives for, 167.

Composite sampling, accuracy of, 167 ; use of drip sample, 162 ; McKay sampler, 165; Michels' cream sampling tube, 165 ; onethird sample pipette, 166 ; Scovell sampling tube, 163 ; tin dipper, 160 .

Composition of butter, 258 ; butter milk, 258; cheese, 258; colostrum milk, 258; condensed 
milk, 258 ; cream, 258 ; milk, 18 , 258 ; milk ash, 17 ; skim milk, 258 ; whey, 258.

Condensed milk, 22 ; analysis of, 229 ; composition of, 258 ; determination of concentration, 230 ; of sp. gr. of, 230 ; testing of, 98 .

Control samples of milk, 111 .

Conversion factor for butter fat, 196.

Conversion tables for thermometer scales, 282 ; for weights and measures, 284.

Cornell test for determining water in butter, 237.

Cows, number of tests required in testing, 147 ; single, sampling milk of, 150 ; when to test, 149.

Cream, 19, 228 ; acidity of, 125 , 129 ; Babcock test for, 75,180 ; bottles, 79 ; care in sampling, necessity of, 182 ; clotted, 255 ; definition, 255 ; determination of acidity of, 125,135 ; errors of measuring in testing, 76 ; evaporated, 255 ; fat in 1 to 1000 lbs., testing 12 to 50 per cent., 270 ; gelatin in, detection of, 249 ; overrun, 194 ; pasteurized, detection of, 248 ; scales, 80 ; separator, 19; gathering and sampling, 185; separation of, influence of temperature, 185 ; sour, determination of acidity, 125 ; spaces, 176 ; specific gravity, 77, 102; standard, 255 ; starch in, 250; testing, 75; eliminating meniscus in, 87; testing outfit, 181 ; testing at creameries, 176 ; tests, correct readings of, 85,87 ; use of 5 cc. pipette in sampling, 86 ; use of milk test bottles, 84 ; test bottles, 79 ; weight of, delivered by 17.6 cc. pipette, 77.

Creameries, calculating dividends at, 203 ; co-operative, 205, 285 . cream testing at, 176 ; proprietary, 204.

Creamery inch, 1, 177.

Curd test, the Wisconsin improved, 137.

Dean's method for determining water in butter, 237 .

Definitions of milk and its products, 254.

DeLaval's butyrometer, 8 .

Devarda's acidimeter, 124.

Diameter of tester and speed required, relation between, 57 .

Dividends, calculating at cheese factories, 215 ; at creameries, 205 ; of both milk and cream at the same factory, 211.

Dividers, use of, 37 .

Double-necked test bottles, 92 ; value of divisions of, 93 .

Draining-rack for test bottles, 42 .

Eichler's Säurepillen, 125.

Expansion coefficient of butter fat. 36.

Failyer and Willard's test, 4.

Farrington's alkaline tablet test, 124.

Fat, 11; color of, an index to strength of acid used, 67 ; content, causes of variation in, 146 ; determination of, in butter, 231 ; in cheese, 244; in milk, 221; globules, 11; Gottlieb's method for determining, 222 ; influence of temperature on separation of, 69 ; measuring of, in cream testing, 86 ; in milk testing, 35 ; pounds in 1-10,000 lbs. of milk, testing, 3 to 5.35 per cent., 266 ; speed required for complete sep aration of, 59 .

Fat-saturated alcohol, use in cream testing, 88 .

Fermentation test, the, 139 .

Filled cheese, detection of, 245 .

"Fitch's Salt Analysis," 239. 
Fjord's centrifugal cream test, 8 . Fluorids, detection of, 252.

Food, influence of on quality of milk, 144, 146, 150.

Food standards, Government, 254.

Fool pipettes, 46.

Formaldehyd, detection of, 252.

Frozen milk, sampling of, 27.

Gauges of cream, 176.

Gelatine in cream, detection of, 249.

Gerber's acid-butyrometer, 7 ;- fermentation test, 139.

Glassware used in the Babcock test, 37 ; calibration of, 48 .

Globulin, 15.

Glycerids of fatty acids, 13 .

Glymol, use in cream testing, 88.

Goat cheese, 14.

Gottlieb method, the, 222.

Government food standards, 254.

Gray's test for water in butter, 235.

Grain-feeding, heavy, influence of, on quality of milk, 155.

Hand separator cream, gathering and sampling, 185.

Hand testers, 60.

Hart's test for casein in milk, 226.

Hemi-albumose, 14.

Herd milk, variations in, 153 ; ranges in variations of, 154 .

Hydrostatic balance, 82.

Iypoxanthin, 18.

Ice-cream, test of, 100 ; definitions, 255.

Immersion refractometer, use of for detection of watered milk, 245.

Introduction, 1.

Iowa station test, $\mathbf{5}$.

Irish test for water in butter, 237 . Kumiss, 256.

Lactic acid in milk, 16.
Lactocrite, 5,7 .

Lactose, 15.

Lactochrome, 18.

Lactometer, the, and its application, 102 ; bi-chromate, influence on, 108; cleaning of, 108 ; degrees, 101 ; N. Y. board of health, 106, 261; Quevenne, 103 ; reading the, 106 ; testing accuracy of, 108; time of taking readings, 107 .

Lecithin in milk, 18.

Leffmann and Beam test, 5 .

Legal standards for milk, 112, 259.

Liebermann's metbod, 5 .

Macroscopic impurities in milk, 250.

Manns' test, 121; testing outfit, 124.

Marschall acid test, 131; rennet test, 141.

McKay sampling tube, 165.

Measuring fat column in testing cream, 86,87 ; in testing milk, 35.

Mercury, calibration with, $\overline{5}$; cleaning, 52.

Metric and customary systems of weights and measures, comparison of, 284.

Michels' cream sampling tube, 165.

Milk, acidity of, 119,132 ; albumen in, 12 ; adulteration - of, 111; amphoteric reaction of, 119 ; ash, composition of, 17 ; blended, definition, 254 ; boiled, detection of, 249 ; casein in, 13 ; chemical analysis of, 217 ; cholesterin in, 18; churned, sampling of, 24 ; citric acid in, 18 ; colostrum, 18; composition of, $10,18,258$; composite sampling of, 160 ; condensed, 22 , 98 , 258; correction table for specific gravity, 262; definitions, 254; detection of coloring matter, 247 ; of preservatives, 135,251 ; determination of acid- 
ity, 132; of ash, 227 ; of casein and albumen, 223 ; of fat, 221 ; of milk sugar, 226 ; of solids, 221 ; of specific gravity, 21T; of water, 220 ; fat in, 11 ; from cows in heat, 112 ; from sick cows, 112; from single cows, sampling of, 150 ; variations in, 142 ; frozen, sampling of, 27 ; gases, 18 ; hypoxanthin, 18 ; lactochrome, 18 ; lactose, 15 ; lecithin, 18; legal standards, 112, 259; macroscopic impurities, 250 ; mineral components, 17; partially churned, sampling of, 24 ; pasteurized, detection, 248 ; preservatives, detection, $\mathbf{1 3 6}$, 251; quality of, influence of food, 155 ; of heavy grain feeding, 155 ; of pasture, 156 ; method of improving, 158; sampling, 23, 29 ; scale, Richmond's, 110 ; scales, 150 ; serum, 10 ; skimming, 116 ; solid, 10 : calculation of, 109 ; specific gravity of, 113 ; souring of, 15 ; sour, sampling of, 26 ; standards, 112 , 254 ; sugar, 15 ; tests for adulteration: nitric acid test, 245 ; sp. gr. of skim milk, milk serum, or whey, 247; testing on the farm, 142; testing purity of, 137 ; urea, 18 ; water, 11 ; watering of, 116 ; detection of, by refractometer, 245 ; watering and skimming, 117.

Milk test, a practical, need of, 1 ; requirements of, 6 ; bottle, use of, in testing cream, 84 ; Russian, 71 .

Milk tests, Babcock, 4, 6; Beimling (Leffmann and Beam), 5 ; Cochran, 5; DeLaval butyrometer, 8; Failyer and Willard, 4 ; Fjord, 8; foreign, 7 ; Gerber acid-butyrometer, 7 ; introduction of, 4 ; lactocrite, 5,7 ; Liebermann, 5 ; Lindstrom, 9 ; Nahm, 5; Parson, 4; Patrick
(Iowa station test), 5; RüseGottlieb, 5, 222 ; sal-method, 5 ; Schmied, 5 ; Short, 4 ; sin-acid, 7 ; Thörner, 5 ; Wollny refractometer, 9.

Milk products, composition of, 19 , 258.

Monrad rennet test, the, 140.

Milk testing, 28 ; on the farm, 142. Mitchell-Walker test, 236.

Nahm's test, 5.

N. Y. board of health lactometer, 105; degrees corresponding to Quevenne lactometer degrees, 261.

Nitric acid test for adulteration of milk, 245.

Non-fatty milk solids, 10.

Normal solutions, 121.

Nuclein, 14.

Official tests of cows, 150.

Oil-test churn, 2, 177.

Oleomargarine, detection of, 240 , 242 ; cheese, detection of, 245 ; tests for artificial coloring matter in, 243.

One-third sampling pipette, use of, 166.

Organization of co-operative creameries and cheese factories, suggestions concerning, 285.

Overrun, 190 ; calculation of, 195 ; factors influencing, 190 ; table, 199, 280; from cream, 194; from milk, 190.

Parsons' test, 5.

Pasteurized milk or cream, detection of, 248.

Pasture, influence on quality of milk, 156.

Patrick's test, 5 ; method for determining water in butter, 236 .

Percentages, average, methods of calculation, 172 ; fallacy of averaging, 171.

Phenolphtalein, 122. 
Physician's centrifuge, use of, in milk testing, 72 .

Pipettes, 30 . 45 ; proper construction of points, 45 ; proper method of emptying, 31 ; calibration, 54.

Pooling system, 3 .

Potassium bi-chromate, 168.

Power testers, 61.

Preservaline, 135, 251; detection of in milk, 135, 251.

Preservatives, for composite samples, 167 ; in milk, detection of, $135,251$.

Primost, 14.

Process butter, detection of, 242.

Proteose, 14.

Quevenne lactometer, the, 106 ; degrees corresponding to scale of N. Y. board of health lactometer, 106, 261.

Readings of cream tests, 86 ; of milk tests, 35 .

Recknagel's phenomenon, 107.

Refractometer, Wollny, 9 ; immersion, use of for detection of watered milk, 245.

Reichert number, 242.

Reichert-Wollny method, 241.

Relative-value tables, 209, 274.

Rennet tests, 140.

Renovated butter, detection of, 242 ; boiling test, 242 ; Waterhouse test, 242.

Reservoir for water in Babcock test, 70.

Richmond's milk scale, 110.

Röse-Gottlieb's method, 5, 222.

Russian milk test, the, 71 .

Salicylic acid, detection of, 252.

Salt, estimation in butter, 239.

Sampling cheese, 97 ; milk, 23, 29 ; milk from single cors, 150.

Sampling tube, for cream, 181; McKay, 165 ; Michels, 165 ; Sco-

Scales for weighing cream, 80 ; milk, 150.
Schmied method, the, 5 .

Scovell sampling tube, 163.

Serum solids, 10.

Short's test, 4.

Siegfeld's modification of Babcocts test, 72 .

Sinking fund, 209.

Separator cream, 19.

Skimming of milk, detection of, 116.

Skim milk, 19 ; Babcock test for, 90 ; chemical analysis of, 228 ; composition of, 258 ; condensed, 255 ; definition, 255 ; sp. gr., 102 ; test bottles, 92 .

Solids not fat, 10 ; formulas for calculating 110 ; tables showing, corresponding to $0-6$ per cent. fat and 26-36 lactometer degrees, 263.

Sour milk, sampling, 26 ; analysis, 228.

Space system, the, 176 .

Specific gravity, 102; cylinders, 103,107 ; influence of temperature, 104; of butter fat, determination of, 240 ; of butter milk, 229 ; of condensed milk, 230 ; of milk, 217 ; of milk solids, 113 ; of sour milk, 229 ; temperature correction table, 262.

Speed required for complete separation of fat, 57; ascertaining necessary speed in Bagcock test, 59.

Spillman's cylinder, 131.

Standard measure for calibrating test bottles, 50 .

Standards of purity, Government, for milk and its products, 254.

Starch in cream, 250.

Steam turbine testers, 61 .

Stokes' acidity pellets, 125.

Storch's test, 248.

Sulfuric acid, 64; table showing strength of, 67 ; testing strength of, 65 .

Sweetened condensed milk, Babcock test for, 99 . 
Swedish acid bottle, 47.

Swedisb acid tester, 67 .

Tank for cleaning test bottles, 43 .

Temperature of turbine testers, 36 ; of fat when tests are read, 36.

Test bottles, 30,37 ; apparatus for cleaning, 41, 44; calibration, 48; cleaning, 40 ; cream, 79 ; draining-rack for, 42 ; marking, 39 ; skim milk, 92 ; rack for use in creameries and cheese factories, 166 ; tank for cleaning, 43.

Testers, 54 ; ascertaining speed of, 58 ; electrical, 63 ; hand, 60 ; power, 61 .

Testing cows, number of tests required during a period of lactation, 147.

Testing milk and its products, 1 ; on the farm, 142.

Test sample, size of, 153 .

Tests of corrs, official, 150.

Thermometer scales, comparison of, 282.

Thörner's method, 5 .

Total solids in milk, 10 ; determination, 221.

Trowbridge method of calibration, 49.

Turbine testers, 61 ; hot, errors in, 36.

Volatile acids in butter fat, determination, 241.

Wagner skim milk bottle, 94 .

Waste acid jar, 40.
Water, calibration with, 48 ; determination of, in butter, 231, 233 ; in cheese, 243 ; in milk, 220 ; reservoir for, 70 ; to be used in the Babcock test, 69 .

Waterhouse test, 243.

Watering of milk, detection of, 116 ; watering and skimming, detection of, 117.

Weights and measures, comparison of metric and customary, 284.

Westphal balance, 219 .

Whey, 22 ; Babcock test for, 94 ; chemical analysis, 228 ; composition, 258 ; definition, 256.

Winton cream bottle, the, 79 .

Wisconsin creamery butter, summary of analyses, 189.

Wisconsin curd test, the improved, 137.

Wisconsin high-pressure oven test, for water in butter, 238.

Wollny refractometer, 9.

World's Fair breed tests, composition of butter from, 188 ; variation in quality of milk, 153 .

Yield of butter, calculation of, 187, and butter fat test, 187 ; from different grades of milk, 192 ; table showing, from 1 to $10,000 \mathrm{lbs}$. of milk, testing 3 to 5.3 per cent., 278.

Yield of cheese, calculation of, 199 ; relation between, and quality of milk, 200 ; table showing, corresponding to 2.5 to 6 per cent. of fat, with lactometer readings of 26 to 36,281 . 


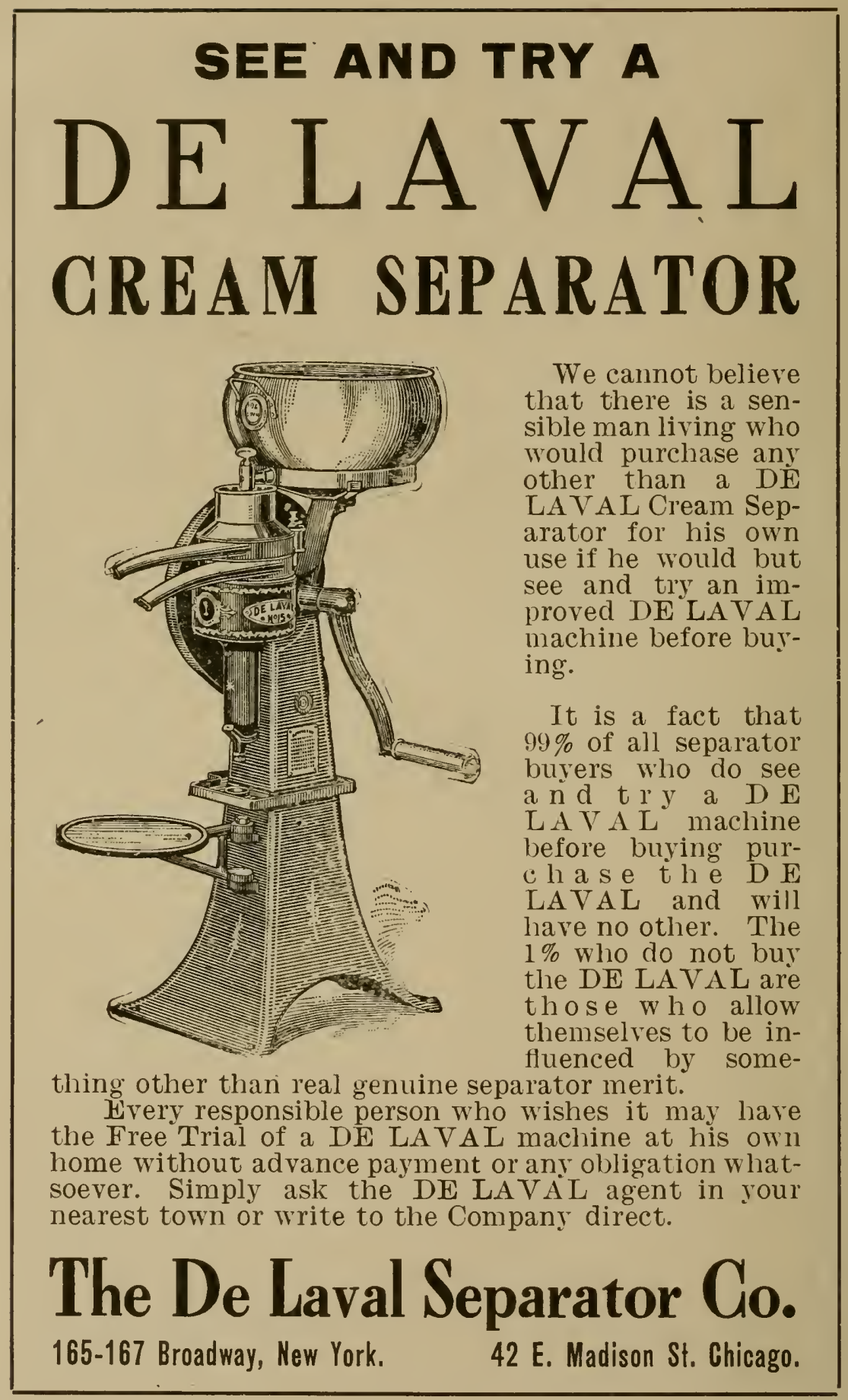




\section{THE "FACILE"}

\section{Iron Frame Babcock Milk Testers}

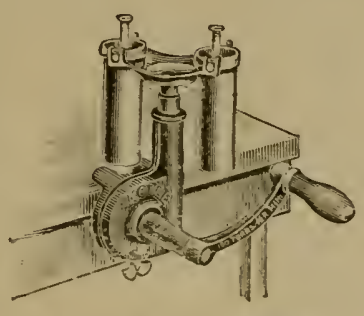

FACILE JR. TESTER

Two Bottle

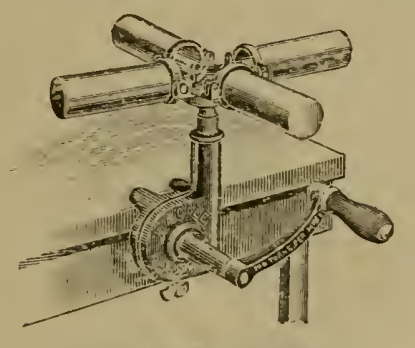

FACILE JR. TESTER

Four Bottle

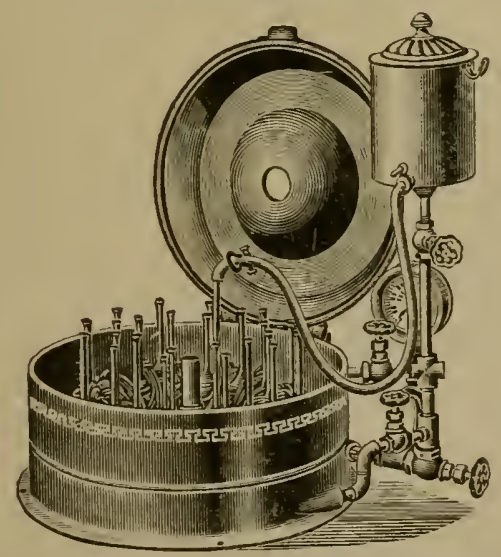

FACILE STEAM TURBINE TESTER 24 Bottle

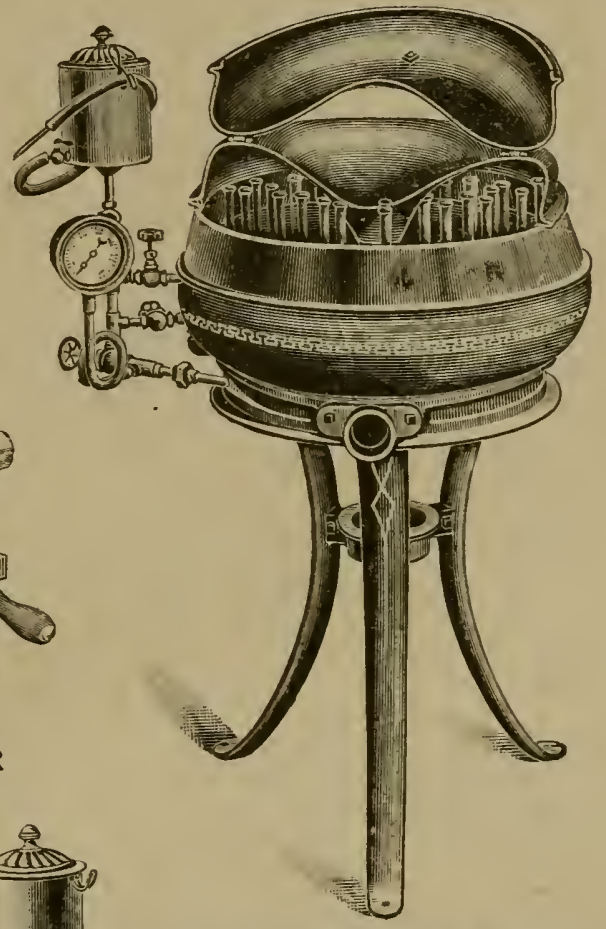

FACILE STEAM TURBINE TESTER 36 Bottle

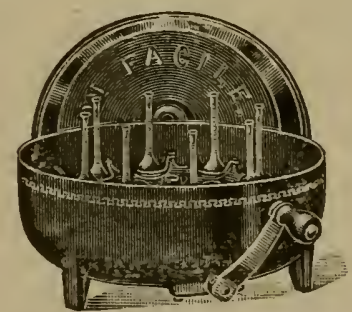

FACILE HAND TESTER Sizes $6,8,10$ and 12 Bottle

\section{H. Burrell \& Co., Little Falls, N. Y.} Creamery, Cheese Factory and Dairy Apparatus and Supplies SEND FOR CATALOGUE 


\section{Testing Specialties}

We furnish everything for the commercial testing of milk and its products. No other concern makes so varied a line. Among our specialties we describe the following:

\section{Wizard Turbine Babcock Tester}

Made for factory use. Enclosed case; top turbine 7 inches in diameter in separate compartment; center spindle has bearings at both ends ; friction brake for stopping; all wearing parts renewable. Made for six and nine inch bottles, 24,32 and 40 bottles in each size, six sizes in all. Cast iron stand furnished if wanted.

\section{0th Century Hand Babcock Tester}

For dairy use and where steam pressure is not available; enclosed case; long center spindle with two bearings; top drive; wearing parts renewable ; noiseless cut gears; made in 6, 8, 10 . 12, and 24 bottle sizes for 6 in. (regular bottles) and in 24 bottle size for 9 in, bottles. We also furnish this tester in aluminum for triaveler's' use.

\section{Electric Drive Babcock Tester}

Same as Wizard and 20th Century tester except that it is driven by electric motor mounted on top of case Furnished in any regular size, and vith motor as required according to the current used.

\section{"Official" Hand Babcock Tester}

For dairymen and travelers. Made to clamp to table or bench or may be permanently fastened with screws; bottles set in deep brass pockets; noiseless cut spur and worm drive. Made in two and four bottle sizes.

\section{Hart Casein Test}

Hand power, similar to 20th Century tester; made for six and twelve tubes. Furnished with all necessary equipment.

\section{Farrington Moisture Test}

Wisconsin High Pressure Oven principle. Two styles of oven furnished; the Farrington tests from two samples upward at one time; Farrington Junior tests a single sample.

\section{The Ames Moisture Test}

Paraffine method. Complete outfit consists of jacketed paraffine container, aluminum sample cup; high reading thermometer and stand.

\section{P. Salt Test}

A simple, practical and accurate test for the per cent. of salt in butter. Furnished complete with full directions for use.

For circulars and prices on the above and other testing apparatus mention this bonk and address

\section{The Creamery Package Mfg. Co. 61.67 West Kinzie Street - Chicago, Illinois}


TORSION BALANGE ENGLOSED BUTTER PRINT SGALE FOR VERIFYING BUTTER PRINTS

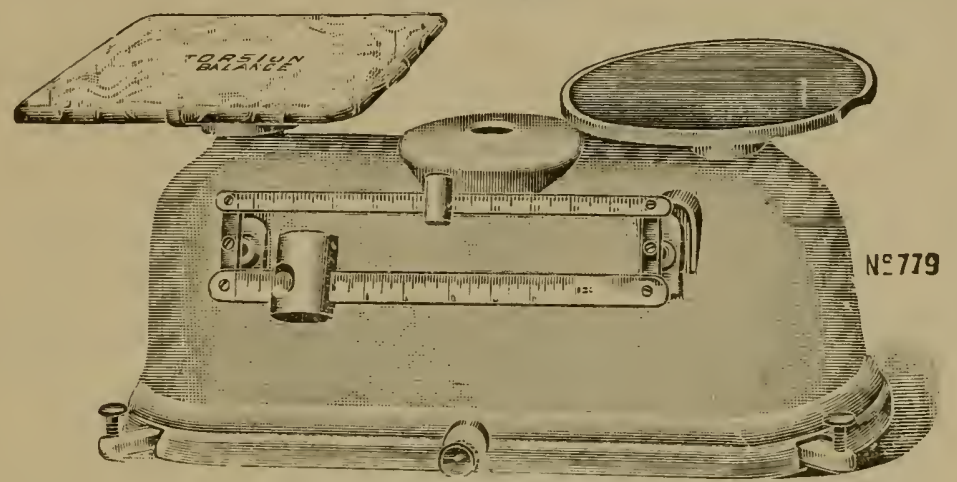

Style No. 779

Metal case and weight plate White Enameled. Lower slide beam graduated to 16 ozs. by $1 / 8$ oz.; upper slide beam operating from the center by $\frac{1}{32}$ oz. on either side. Sensitive to 10 grains. Arrest rod (knob in front of base), locks scale when not in use or when loading. Glass index on top of case. Porcelain plate $8^{\prime \prime} \times 8^{\prime \prime}$.

Measurements:-Base-181/2" L. -7" W. Over all-201/2" L. $-81 / 4$ " W. $9^{\prime \prime}$ High. Write for our catalogue illustrating our full line of cream test and butter test scales.

THE TORSION BALANCE COMPANY

Manufacturers of Cream Test scales for one, two, four and twelve bottle work. FACTỌRY AND SHIPPING ADDRESS, 147-9 Eighth St., Jersey Clty, N. J. Office, 92 Reade St., NEW YORK CITY

\section{GHR. HANSEN'S LABORATORY}

HEADQUARTERS FOR

Dairy Preparations Unequalled in Purity and Strength Chr. Hansen's Danish Rennet Extract Chr. Hansen's Danish Cheese Color

VEGETABLE BUTTER COLOR and LACTIC FERMENT CULTURE

Rennet Tablets and Cheese Color Tablets for CheeseMaking on the Farm

JUNKET GREAM TABLETS for Ice Gream Manufacturers

JUNKET TABLETS for Dainty Desserts

JUNKET BRAND GOLORS and FLAVORS

JUNKET BRAND BUTTERMILK TABLETS

for making Buttermilk

\section{CHR. HANSEN'S LABORATORY, BOX 1140 LITTLE FALLS, N. Y.}




\title{
OLD Standards NEW Standards
}

It is of no importance to you who made the best Rennet Extract years ago, but who is making it to-day, and we have improved every step in the manufacture so that ours is greatly superior to all other brands.

NATIONAL DAIRY SHOW, CHICAGO, 1910

First Prize: American Cheese, Aug. Brandt, Forestville, Wis.

First Prize: Brick Cheese, Jacob Erb, Blue Mounds, Wis. BOTH USED

\section{The Marschall Rennet Extract}

Only Up-to-date Standard of Quality

We have the Largest, most Sanitary and Finest Equipped Dairy

Laboratory in the Country and make the best Rennet Extract and Cheese Color-no exceptions.

THE MARSCHALL DAIRY LABORATORY,

Madison, Wis.

ALL TEXT AND REFERENCE
BOOKS USED IN

\section{American Dairy Schools}

MAY BE OBTAINED FRON

MENDOTA BOOK CO., Madison, Wis.

\author{
See List on Following Page
}

\section{GREAM-WEIG HING SGALE For Use in Connection with the Babcock Test}

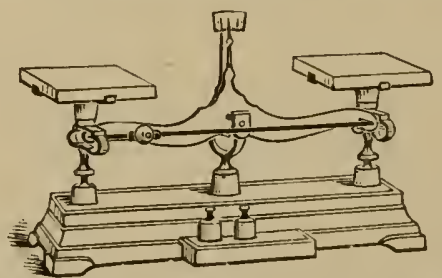

Price $\$ 10 \quad 911$ Arch St., Philadelphia, Pa.

THIS SCALE is especially designed 1 for very accurate weighing of cream, butter and cheese. All bearings are agate: plates are porcelain: base and underconnections are galvanized. making a rust-proof scale. It has a side bar in front to balance the test bottle, and is provided with the necessary weights. Base of scale $10^{1} / 2$ in. long: porcelain plates 3 in. square. Manufactured by

911 arch St., Philadelphia, Pa. HENRY TROEMNER 


\section{BOOIS}

The following books on dairying and related topics will be sent, postage prepaid, on recelpt of the price given.

Farrington-Woll, Testing Milk and Its Products, Twentieth ed. Madison, Wis., 1911, $304 \mathrm{pp} \ldots \ldots \ldots$-_._. 25 Woll, Handbook for Farmers and Dairymen. Fifth ed. New York, 1908, 488 pp___. 150 Grotenfelt-IVoll, Principles of Modern Dairy Practice. Third ed. New York, 1905, $286 \mathrm{pp}$

Wing, Milk and Its Products. Tenth ed. New York, 1906, $311 \mathrm{pp}$

McKay-Larson, Principles and Practice of Butter Making. Second ed. New York, 1908, 351 pp_.._____ 150

Fleischmann, The Book of the Dairy. London and New York, 1896, $344 \mathrm{pp}$

Snyder, Dairy Chemistry. New York, 1906, $190 \mathrm{pp}-1$ - 125

Winslow, The Production and Handling of Clean Milk. New York, 1907, $207 \mathrm{pp}$

Lane, The Business of Dairying. New York, 1909,250 pichels, Creamery Butter-Making. Lafising, Mich., 1904, $271 \mathrm{pp}$

Dean, Canadian Dairying. Toronto, $1903,260 \mathrm{pp}_{-}-{ }_{1} 00$

Russell, Dairy Bacteriology. Fifth ed. Madison, $\Pi$ is.. 1908,214 pp_... 100

Gurler, The Farm Dairy. Chicago, $1909,164 \mathrm{pp}_{-}$

Conn, Practical Dairy Bacteriology. New York, 1907 , 340 pp .

Decker, Cheese Making. Rev, ed. by F. W. Woll. Madison, Wis., 1909, $211 \mathrm{pp}$

Belcher, Clean Milk. New York, $1903,146 \mathrm{pp}$

Monrad, ABC in Buttermaking. Winnetka, Ill., 1900,

$68 \mathrm{pp}-\mathrm{ABC}$ in Cheesemaking. Winnetka, Ill., 1900 ,
Monrad, Schoenman, Butter Fat and Dividend Calculator.--
Anderson, Simplex Creamery Calculator. Minneapolis,

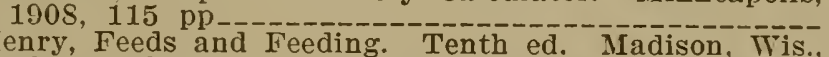
$1910,613 \mathrm{pp}$

Plumb, Types and Breeds of Farm Animals. New York, 1907,563 pp

Jensen, Essentials of Milk Hygiene. Philadelphia, $190 \overline{7}$,

$275 \mathrm{pp}$
Woll, A Book on Silage. Revised ed. Chicago, 1901,
$234 \mathrm{pp}$ raig, Judging Live Stock. Second ed. Des Moines, Ia., $1901,193 \mathrm{pp}$

King, Physics of Agriculture. Fourth ed. Madison, Wis., 1907, $604 \mathrm{pp}$

Boss, Instructions for Traction and Stationary Engineers. Minneapolis, 1906, $319 \mathrm{pp}$

\section{MENDOTA BOOK GO., Madison, Wisconsin}




APR 271911 
One copy del. to Cat. Div.

$$
\text { MAY } 8 \quad 1911
$$




\section{LIBRARY OF CONGRESS}

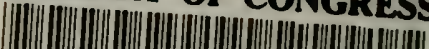

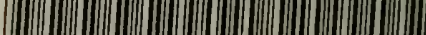

(1)

00008954586 